\title{
CURRENT SOURCE CONVERTER-BASED \\ OFFSHORE WIND FARM: CONFIGURATION, MODULATION, AND CONTROL
}

By

\section{Qiang Wei}

B. Sc., Henan University of Science and Technology, Luoyang, China, 2008

M. Sc., Xi' an Jiaotong University, Xi'an, China, 2012

\author{
A dissertation \\ presented to Ryerson University \\ in partial fulfillment of the \\ requirements for the degree of \\ Doctor of Philosophy \\ in the program of
}

Electrical and Computer Engineering

Toronto, Ontario, Canada, 2017

(C) Qiang Wei, 2017 


\section{Author's Declaration}

I hereby declare that I am the sole author of this dissertation. This is a true copy of the dissertation, including any required final revisions, as accepted by my examiners.

I authorize Ryerson University to lend this dissertation to other institutions or individuals for the purpose of scholarly research.

I further authorize Ryerson University to reproduce this dissertation by photocopying or by other means, in total or in part, at the request of other institutions or individuals for the purpose of scholarly research.

I understand that my dissertation may be made electronically available to the public. 


\title{
Current Source Converter-Based Offshore Wind Farm: Configuration, Modulation, and Control
}

\author{
Qiang Wei \\ Doctor of Philosophy \\ Electrical and Computer Engineering \\ Ryerson University, Toronto, 2017
}

\section{Abstract}

Offshore wind power is attracting increased attention because of considerable wind resources, higher and steadier wind speeds, and smaller environmental impact. Recently, a current source converter (CSC)-based series-connected configuration is proposed and it is considered a promising solution for offshore wind farms as the offshore substation used in existing systems can be eliminated. However, such a CSC-based configuration has disadvantages in terms of size and weight, dynamic performance, cost, reliability, and efficiency. Therefore, this thesis proposes new configurations, modulation scheme, and control schemes to improve the performance of the CSC-based offshore wind farm.

First, a new configuration is proposed for the CSC-based offshore wind farm. Compared with existing CSC-based configurations, the new one is expected to be smaller in size and weight. Second, conventional space vector modulation (SVM) with fast dynamic response cannot be used for grid-side CSCs because of its high-magnitude low-order harmonics. To solve this issue, an advanced SVM with superior low-order harmonics performance is proposed.

Third, power balancing among series-connected CSCs is an important consideration for system reliability. The possible imbalance of power is investigated and quantitatively defined. A power 
balancing scheme is proposed, based on which equal power distribution among CSCs is ensured. Fourth, to lower the system insulation requirement of the CSC-based configuration, a bipolar operation is investigated. Compared with monopolar mode, bipolar mode gives lower insulation level, thus contributing to the system in terms of lower cost and higher reliability for a given power rating. In addition, an optimal dc-link current control giving higher efficiency is proposed for the bipolar system. Fifth, an optimized control strategy with reduced cost and improved efficiency is proposed for the CSC-based offshore wind farm. The nominal number of onshore CSCs is optimized, which reduces the cost on power converters. And an optimized bypass operation is introduced to onshore CSCs, which improves the efficiency of the system.

Finally, simulation and experimental results are provided to verify the performance of the proposed configuration, modulation scheme, and control schemes. 


\section{Acknowledgements}

I would like to express my deep gratitude to my supervisors, Professors Bin $\mathrm{Wu}$ and David $\mathrm{Xu}$

for their invaluable guidance, generous support, and consistent kindness throughout the course of the work.

I would like to thank all my friends in Laboratory of Electric Drives Application and Research (LEDAR), especially Dr. Sixing Du, Dr. Kai Tian, Dr. Chushan Li, Dr. Shuai Wang, Dr. Longcheng Tan, Dr. Yulin Zhong, Dr. Zhijian Fang, and Hang Gao, for many fruitful discussions and joyful time that we shared together.

Special appreciation goes to my dear parents and wife for understanding and supporting me all along. Special thanks to my newborn baby who is the source of my motivation.

Financial supports from Professors Bin Wu, David Xu, Department of ELCE and Ryerson University are gratefully acknowledged. 


\section{Table of Contents}

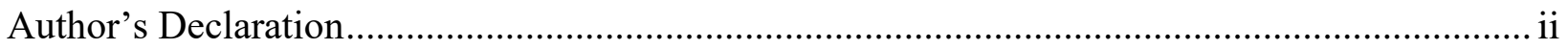

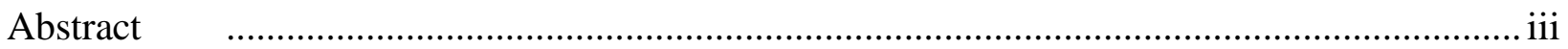

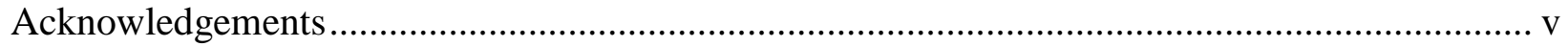

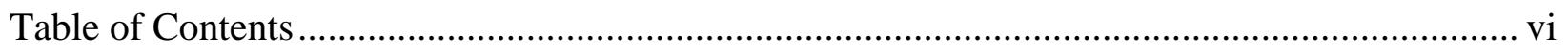

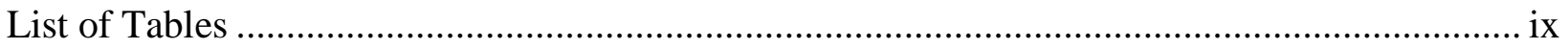

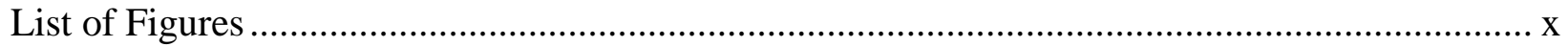

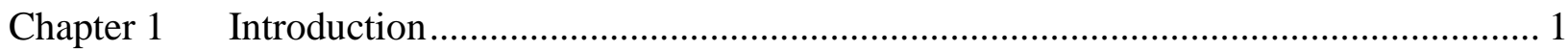

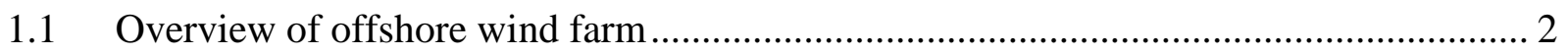

1.2 Megawatt wind energy conversion system …………............................................ 3

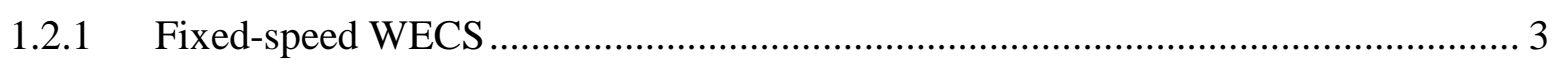

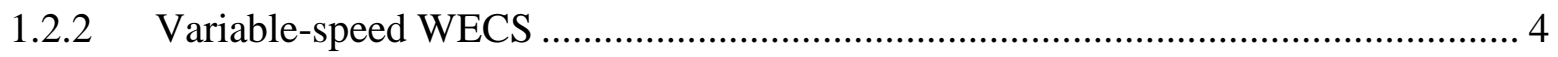

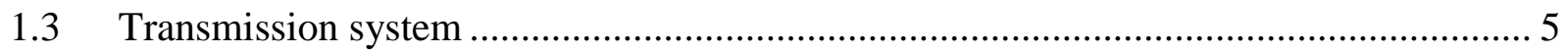

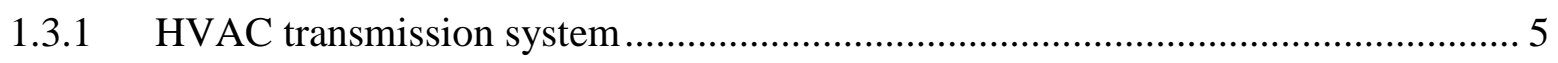

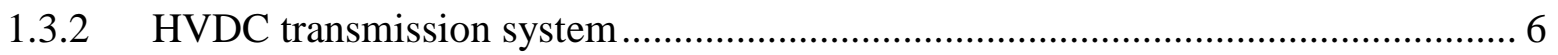

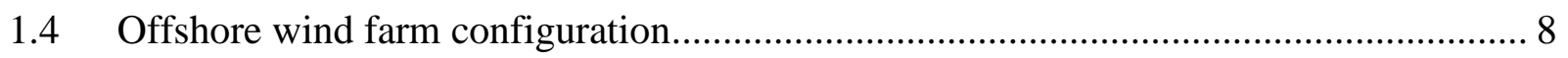

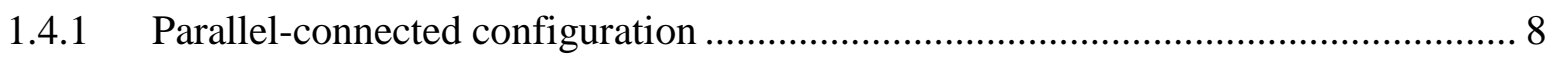

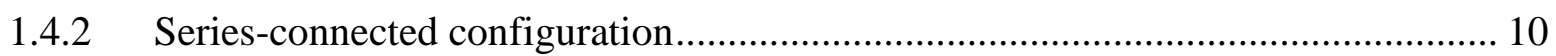

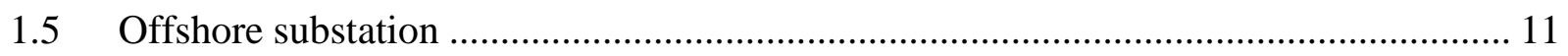

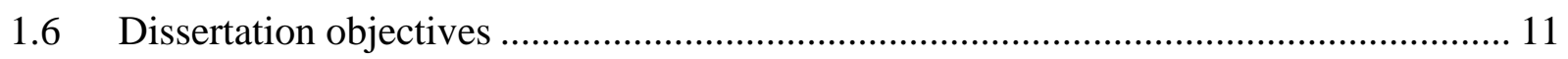

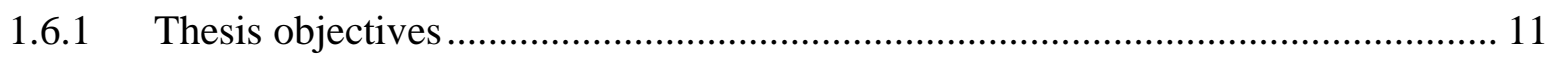

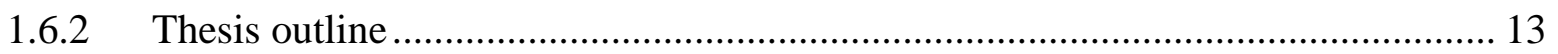

Chapter 2 Medium-Frequency Transformer Based Offshore Wind Farm Configuration ........ 15

2.1 Medium-frequency transformer based configuration................................................. 15

2.1.1 Configuration with medium-voltage turbine ................................................... 16

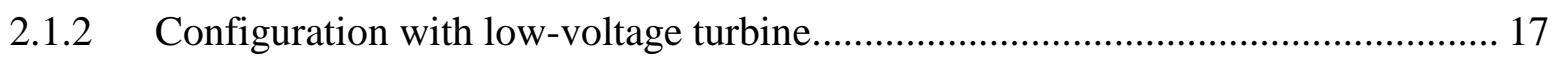

2.1.3 Medium-frequency transformer .................................................................... 18

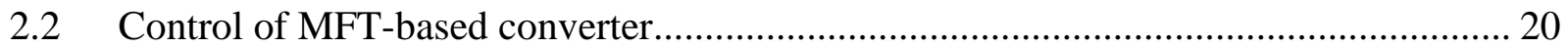

2.2.1 Voltage balance control for MV configuration.................................................. 20

2.2.2 Current balance control for LV configuration .................................................. 23

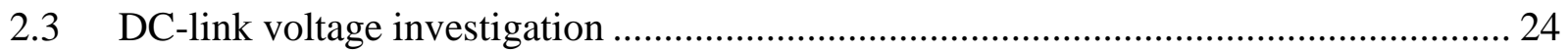

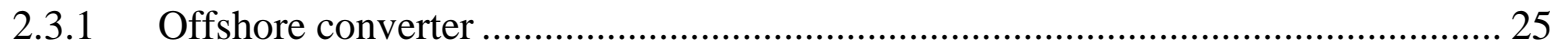




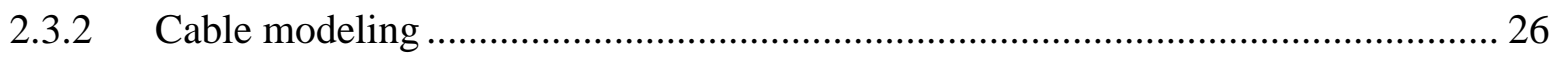

2.3.3 DC-link voltage ........................................................................................ 30

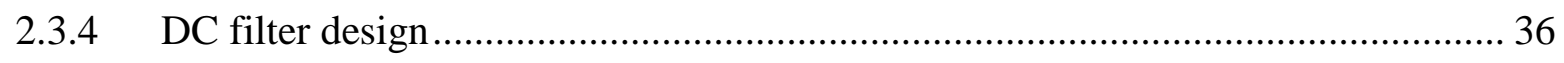

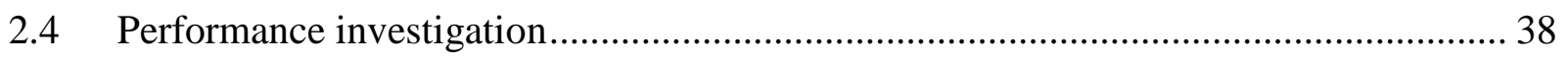

2.4.1 Voltage balance control for MV configuration.................................................... 38

2.4.2 Current balance control for LV configuration .................................................. 42

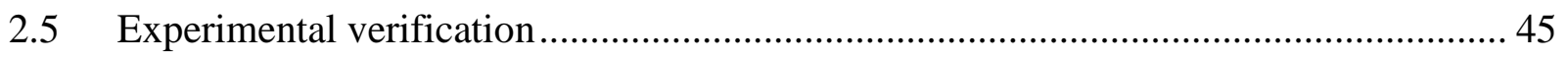

2.5.1 Experimental verification for MV configuration .................................................. 45

2.5.2 Experimental verification for LV configuration .................................................... 49

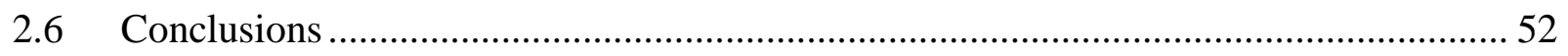

Chapter 3 Natural Sampling SVM with Superior Harmonics Performance for CSCs ............. 53

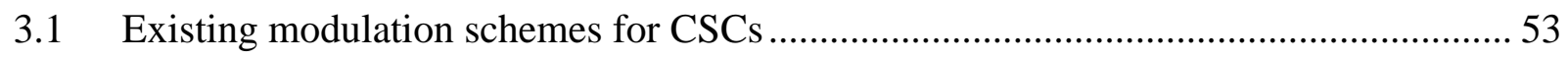

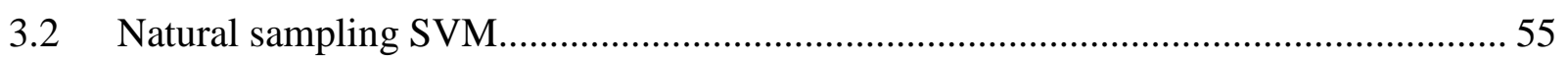

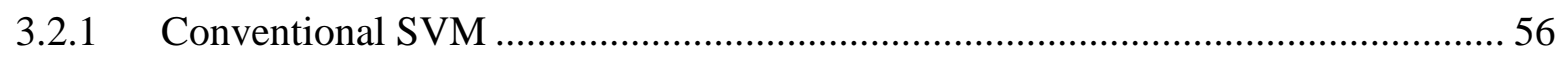

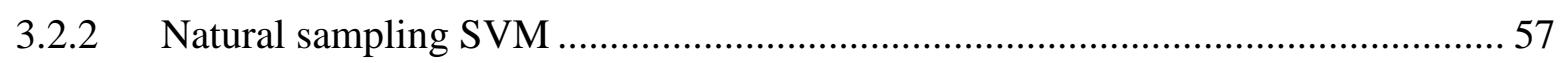

3.2.3 Comparison between conventional SVM and NS-SVM ...................................... 59

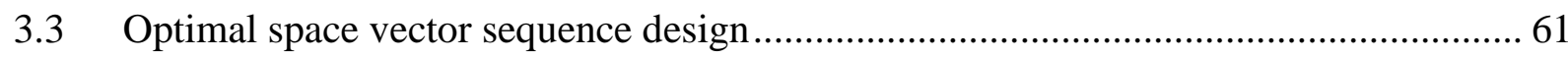

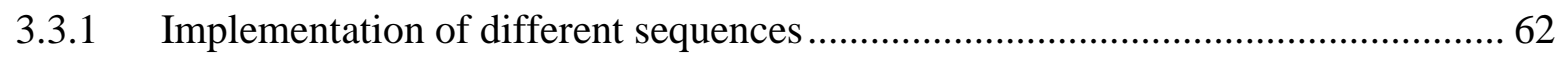

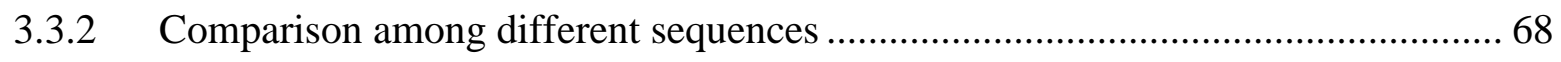

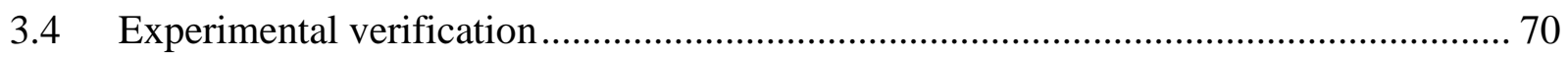

3.4.1 Comparison between conventional SVM and NS-SVM ………………….......... 70

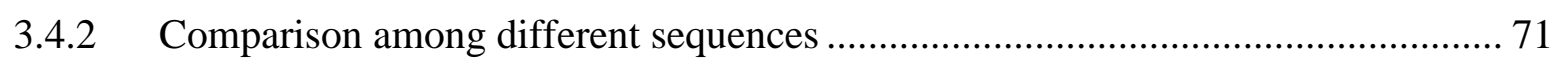

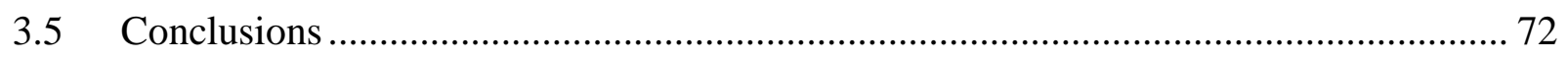

Chapter 4 Power Balancing Control of Series-Connected CSCs ……………...................... 74

4.1 Control objectives of series-connected CSCs ............................................................. 74

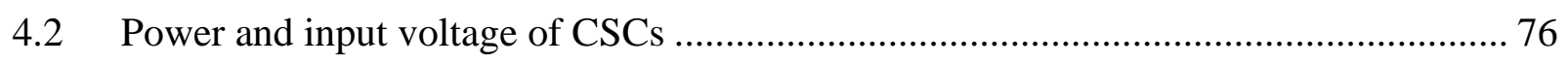

4.2.1 Relationship between power and input voltage .................................................. 76

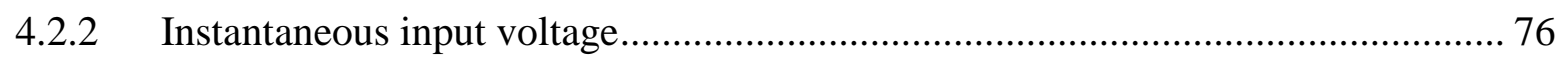

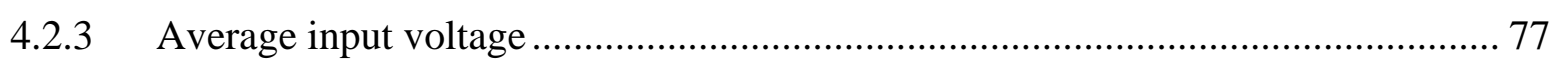

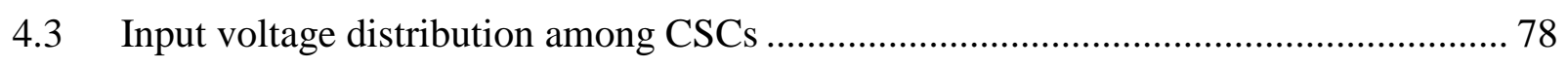

4.3.1 Voltage distribution under practical conditions .................................................... 78

4.3.2 Voltage distribution under power factor control................................................. 81

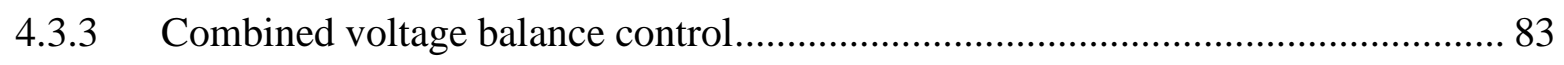

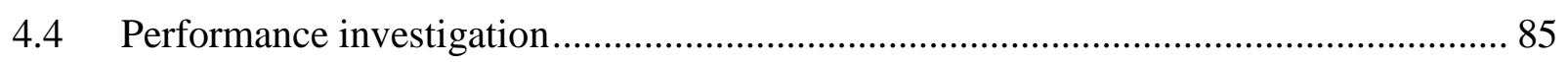




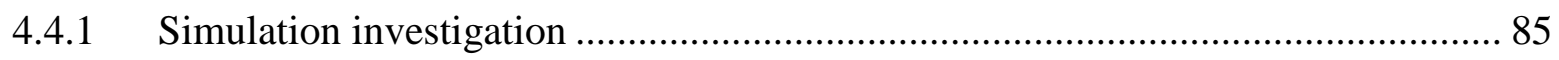

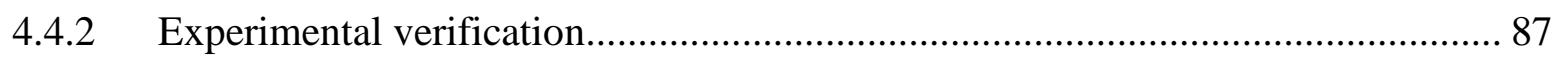

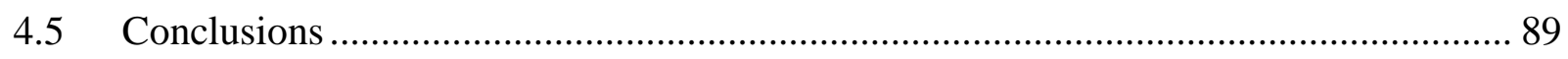

Chapter 5 DC-link Current Control of Offshore Wind Farm with Bipolar HVDC System .... 91

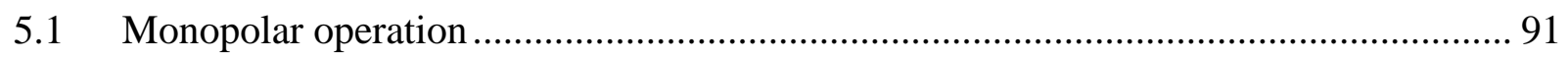

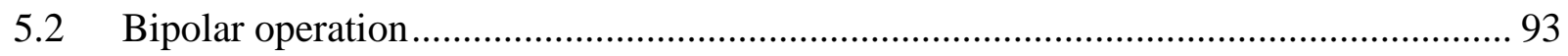

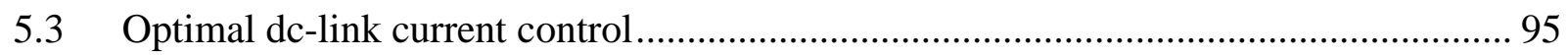

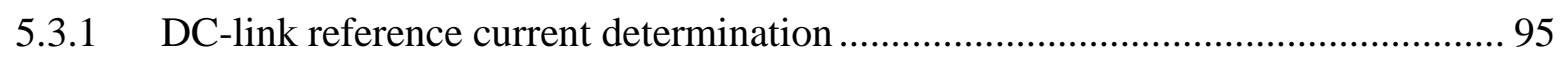

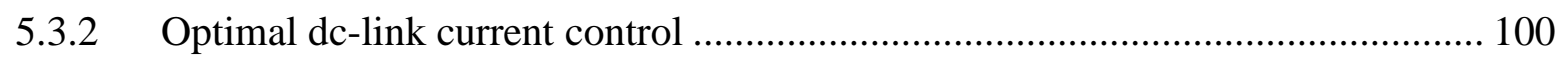

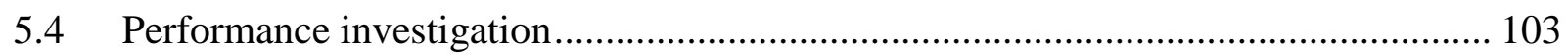

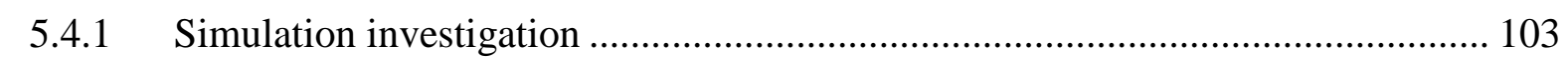

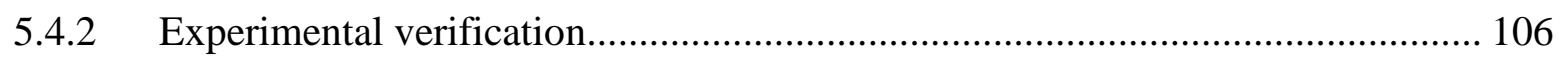

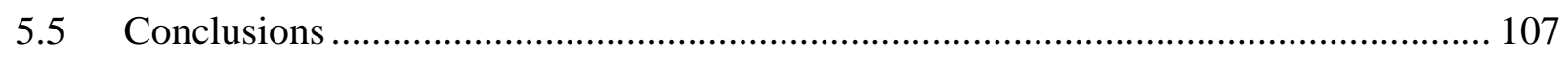

Chapter 6 Optimized Control Strategy for Offshore Wind Farm with Reduced Cost and

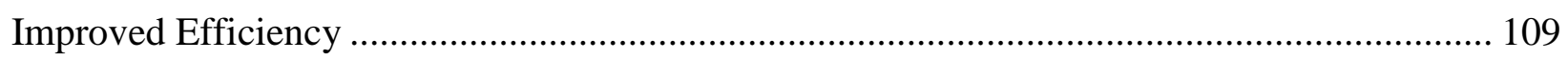

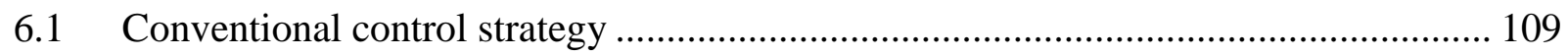

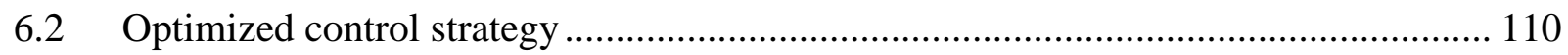

6.2.1 Optimized nominal number of CSCs with lower cost and higher efficiency ....... 111

6.2.2 Optimized control of CSCs with higher efficiency.............................................. 114

6.2.3 Overall strategy of the wind farm ................................................................ 118

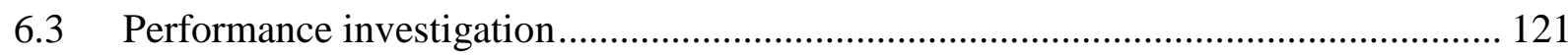

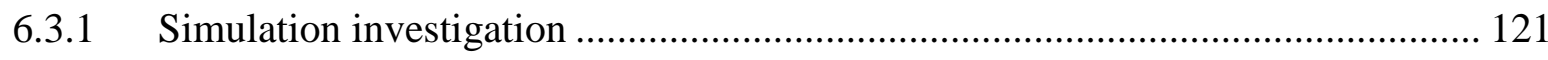

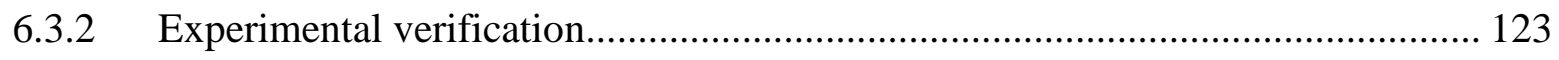

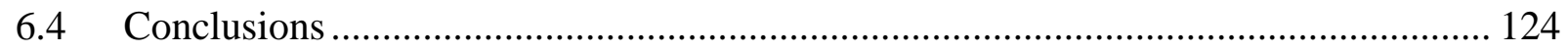

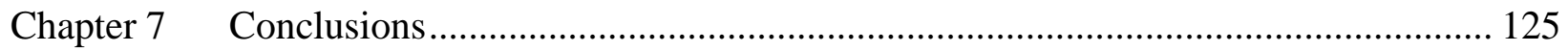

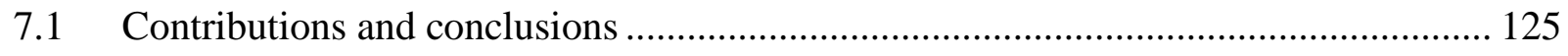

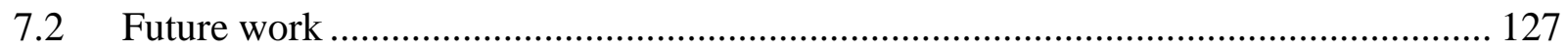

Appendix System Parameters in Simulations and Experiments.......................................... 128

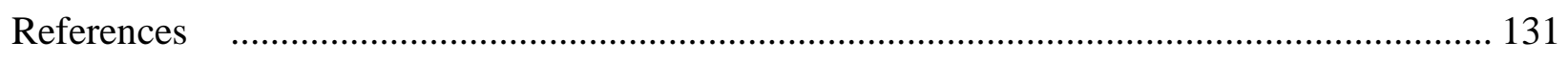

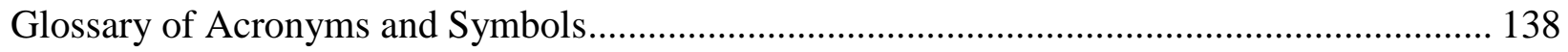




\section{List of Tables}

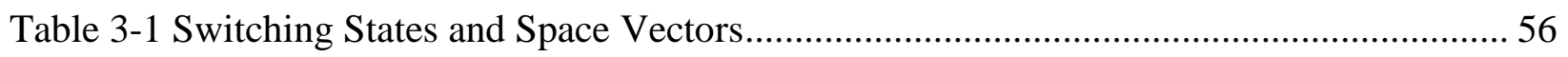

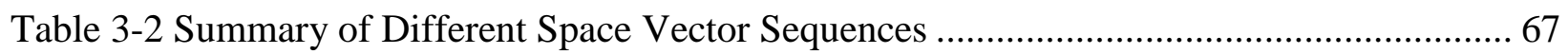

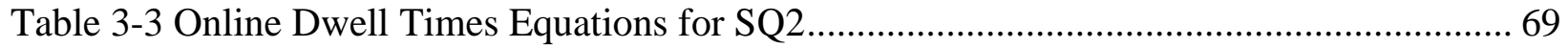

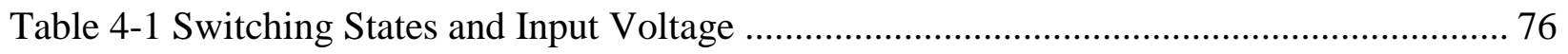

Table 6-1 Comparison Between Conventional and Proposed Schemes ................................ 116

Table 6-2 Comparisons Between Conventional and Proposed Strategies .............................. 117

Table A-1 Simulation and Experimental Parameters......................................................... 128 


\section{List of Figures}

Figure 1-1 Basic structure of offshore wind farm [7], [8]. .............................................. 3

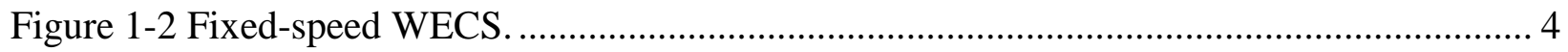

Figure 1-3 DFIG-based indirect-drive WECS. .................................................................. 5

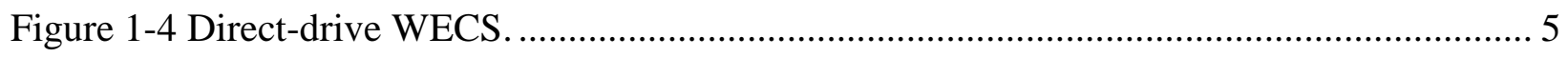

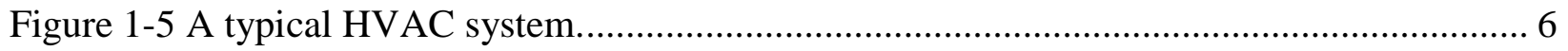

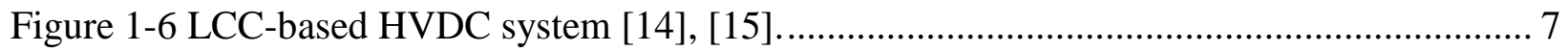

Figure 1-7 VSC-based HVDC system [16], [17] ................................................................ 7

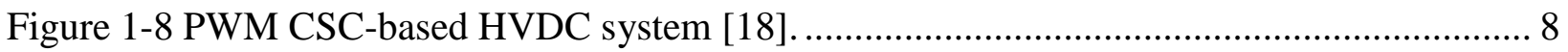

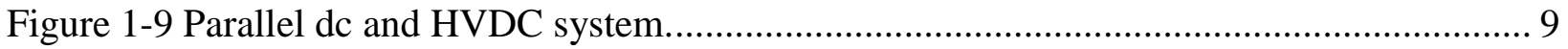

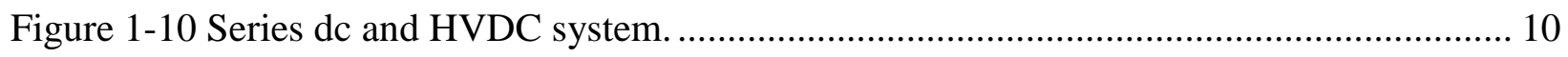

Figure 1-11 Offshore substation used in "Dolwin2" by ABB [35] ......................................... 11

Figure 2-1 CSC-based series-connected offshore wind farm. ............................................ 16

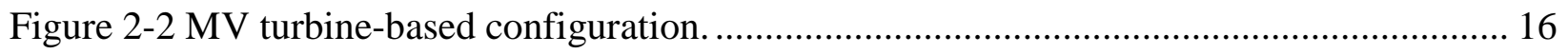

Figure 2-3 Proposed LV turbine-based configuration. ..................................................... 18

Figure 2-4 Power-electronic traction transformer system by ABB [42], [43] ........................... 18

Figure 2-5 Voltage balance control for MV configuration................................................ 21

Figure 2-6 Equivalent circuit used for stability analysis. ................................................. 22

Figure 2-7 Average current balance control for LV configuration......................................... 24

Figure 2-8 Key waveforms of each offshore converter. ................................................. 25

Figure 2-9 Pulse-wave voltage source. (a) time domain. (b) frequency domain...................... 26

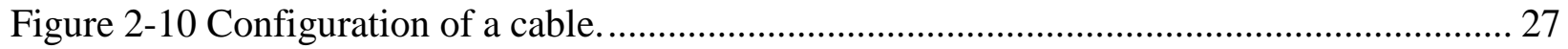

Figure 2-11 LRC sections-based single-core cable. ............................................................. 28

Figure 2-12 Magnitude response of the transfer function of a $1 \mathrm{~km}$ cable.............................. 29

Figure 2-13 Magnitude response of the transfer function of $50 \mathrm{~km}$ and $48 \mathrm{~km}$ cables. ............. 29

Figure 2-14 Configuration when evenly placing dc-link inductor at offshore side................... 30

Figure 2-15 Equivalent circuit of Figure 2-14 ............................................................. 31

Figure 2-16 Equivalent circuit of the system when placing dc-link inductor at onshore station.. 31

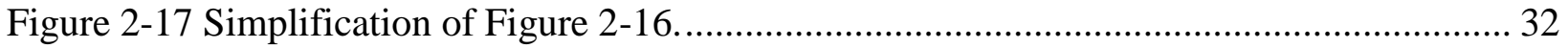




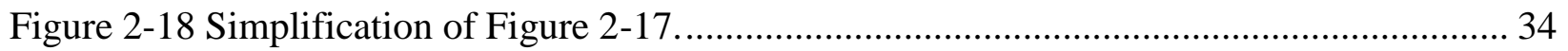

Figure 2-19 DC-link voltage and dc-link current under the condition of (2-18)........................ 35

Figure 2-20 DC-link voltage and dc-link current without the condition of (2-18), ....................... 35

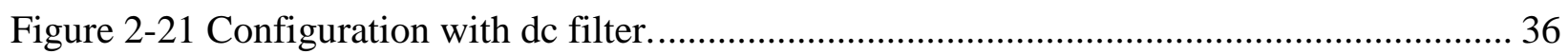

Figure 2-22 Voltage across each offshore converter. ……......................................................... 37

Figure 2-23 DC-link voltage and dc-link current with DC filter................................................. 38

Figure 2-24 Simulated performance with and without voltage balance control.......................... 39

Figure 2-25 Simulated performance under stepped wind speed................................................. 40

Figure 2-26 Simulated performance under reactive power control. .............................................. 41

Figure 2-27 Grid injected current and phase voltage............................................................ 42

Figure 2-28 Simulated performance with/without current balance control................................. 42

Figure 2-29 Simulated performance under stepped wind speed................................................... 43

Figure 2-30 Simulated performance under stepped reactive power control................................. 44

Figure 2-31 Experimental performance with/without voltage balance control. ............................ 46

Figure 2-32 Simulated performance under stepped input voltage ............................................... 47

Figure 2-33 Experimental performance under reactive power control........................................ 48

Figure 2-34 Experimental performance with/without average current sharing control................ 49

Figure 2-35 Experimental performance under stepped input voltage......................................... 50

Figure 2-36 Experimental performance under stepped reactive power control............................ 51

Figure 3-1 Topology of grid-side CSC. ................................................................................ 54

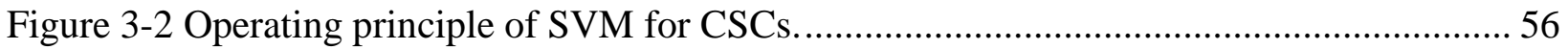

Figure 3-3 Dwell time calculation of conventional SVM and NS-SVM..................................... 57

Figure 3-4 Flow chart of dwell times solving based on two-step iterations................................ 59

Figure 3-5 Harmonic profile of output PWM current based on conventional SVM. ................... 60

Figure 3-6 Harmonic profile of output PWM current based on NS-SVM. ................................. 60

Figure 3-7 Low-order harmonics performance between conventional SVM and NS-SVM. ........ 61

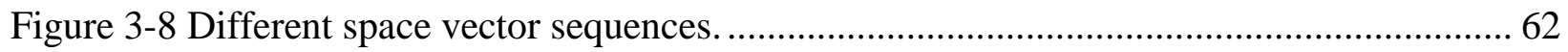

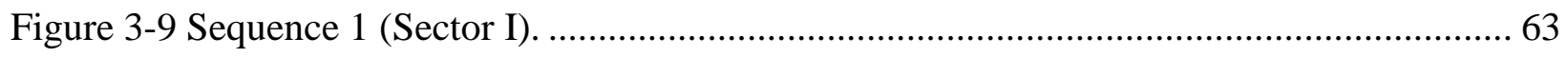

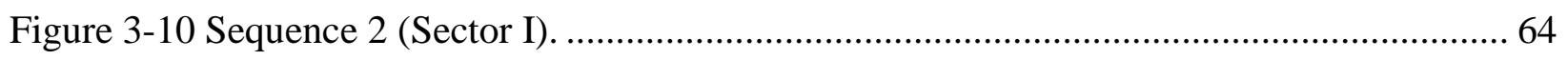

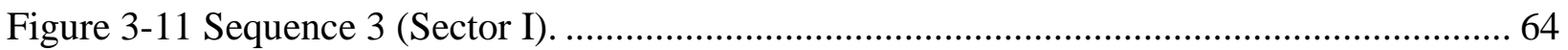

Figure 3-12 Sequence 4. (a) Sector I, III, V. (b) Sector II, IV, VI............................................... 65 


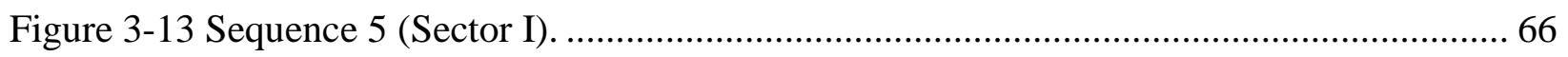

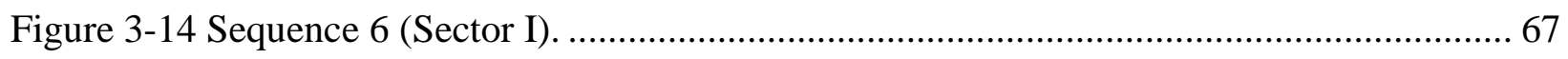

Figure 3-15 Low-order harmonics performance among different sequences................................. 68

Figure 3-16 Comparison of low-order harmonics between one-step and two-step equations...... 69

Figure 3-17 Experimental waveforms based on conventional SVM.......................................... 70

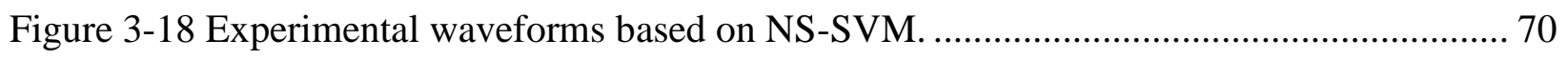

Figure 3-19 Experimental waveforms of different sequences under unity modulation index...... 71

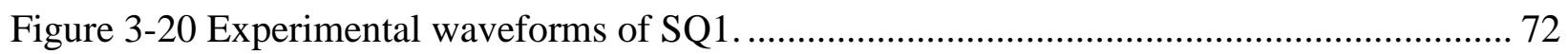

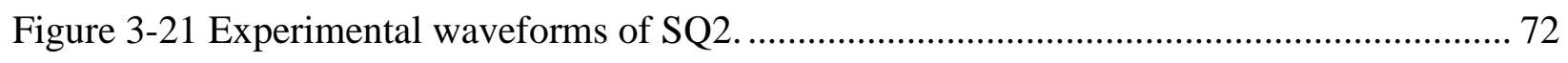

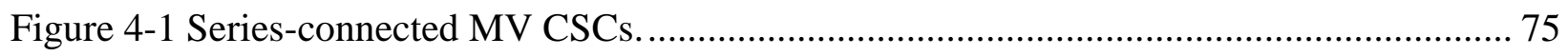

Figure 4-2 Conventional control scheme for series-connected CSCs [40]................................ 75

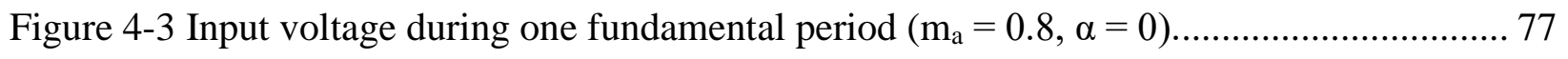

Figure 4-4 Phasor diagram under different conditions. .......................................................... 79

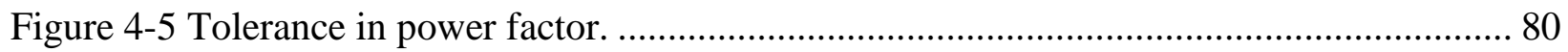

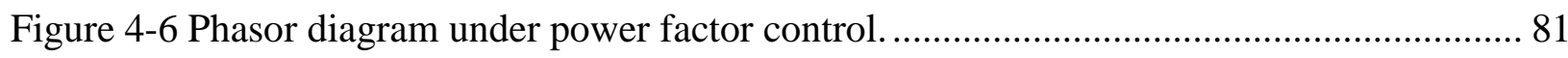

Figure 4-7 Input average voltage under UPF control. ............................................................. 83

Figure 4-8 Proposed combined voltage balance control........................................................... 84

Figure 4-9 Phasor diagram based on the proposed combined control scheme ............................ 85

Figure 4-10 Simulated performance with and without voltage balance control........................... 86

Figure 4-11 Simulated performance under stepped dc-link current. .......................................... 87

Figure 4-12 Experimental performance with and without voltage balance scheme..................... 88

Figure 4-13 Experimental performance under stepped dc-link current. ........................................ 89

Figure 5-1 CSC-based offshore wind farm with monopolar HVDC system................................ 92

Figure 5-2 CSC-based offshore wind farm with bipolar HVDC system..................................... 93

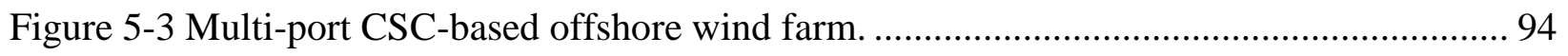

Figure 5-4 Path for dc-link current under monopolar and bipolar modes. ................................... 95

Figure 5-5 A simplified modular MFT-based converter for unit \#n............................................. 96

Figure 5-6 Reference dc-link current determined by generator-side converter............................ 98

Figure 5-7 Reference dc-link current determined by generator- and grid-side converters......... 100

Figure 5-8 Bipolar offshore wind farm with conventional dc-link current control. .................... 101

Figure 5-9 Bipolar offshore wind farm with the optimal dc-link current control........................ 102 
Figure 5-10 Simulated performance of the bipolar system under stepped dc-link current........ 104

Figure 5-11 Simulated performance of the bipolar system when one module is bypassed........ 105

Figure 5-12 Experimental performance of the bipolar system under stepped dc-link currents. . 106

Figure 5-13 Experimental performance of the bipolar system when one module is bypassed... 107

Figure 6-1 CSC-based offshore wind farm with different wind speeds. ................................ 110

Figure 6-2 Final dc-link reference current determination............................................... 111

Figure 6-3 Equivalent circuit of a CSC used for loss evaluation........................................... 113

Figure 6-4 Overall control strategy of the CSC-based offshore wind farm........................... 120

Figure 6-5 Simulated performance of the two-CSC system under optimized control............... 121

Figure 6-6 Experimental performance of the optimized strategy. ..................................... 122

Figure 6-7 Gating Signals of switching devices. ........................................................... 123

Figure A-1 Photo of the lab-scaled platform. ................................................................ 130

Figure A-2 10-kVA back-to-back PWM CSC converter..................................................... 130 



\section{Chapter 1}

\section{Introduction}

Among all available renewable energy sources, wind energy (onshore and offshore) has increasingly become a mainstream [1]. Compared with onshore wind power, the offshore wind power features considerable wind resources, higher and steadier wind speed, and minimized environmental impact [2].

Generally, there are two types of configurations, namely, fixed-speed and variable-speed configurations for single-turbine wind energy conversion systems (WECS) [2]. In the case of the fixed-speed system, generator terminals are directly connected to the grid with no power converter being required. On the contrary, variable-speed system employs power converters for adjusting the generator speed to capture the maximum power from the wind. The variable-speed operation of the turbine gives higher energy efficiency and lower mechanical stress, thus becoming the dominant technology in WECS [3].

A group of wind turbines are placed over an extended area to form a wind farm. In general, the whole wind farm consists of two parts. One is the power generation system, and the other is the power transmission system. The power generation system is to generate and collect the captured wind power of the wind farm which is then transmitted to the onshore grid system through the transmission system. The power transmission system includes high voltage ac (HVAC) and dc (HVDC) transmission systems. By contrast to HVAC which is most suitable for applications where the distance is lower than around $50 \mathrm{~km}$, HVDC is dominating the market when the transmission distance is above $50 \mathrm{~km} \mathrm{[4],} \mathrm{[5].}$

The initial and maintenance costs of offshore wind farms are higher compared to onshore farms because stronger foundations are needed. Therefore, aside from considering reliability and efficiency as the main requisites for all onshore systems, the footprints and weights of the components are particularly important for offshore systems. The total weight of the system that is dominated by the offshore substation significantly affects the cost and complexity of the offshore wind farm [6]. 
In this thesis, new configurations are proposed for offshore wind farms with smaller size and weight. Besides, advanced modulation scheme and optimized control schemes are proposed to improve the performance of the proposed configurations in terms of lower cost, higher reliability, and higher efficiency and flexibility.

This chapter starts with a comprehensive review of the offshore wind farm system including the basic electrical structure, megawatt WECS in offshore applications, transmission systems, offshore wind farm configurations, and offshore substation. Following the review, the objectives of the dissertation are defined.

\subsection{Overview of offshore wind farm}

Figure 1-1 shows the basic structure of a typical offshore wind farm where only main electrical components are considered [7]. As shown in Figure 1-1, the output of the offshore converter, that is normally $690 \mathrm{~V}$, is stepped up to $33 \mathrm{kV}$ by a step-up transformer, then converted to an HVAC level by another step-up transformer, and then converted to an HVDC level by an ac/dc power converter (modular multilevel converters (MMC) is dominating the market) [8]. The captured power of the wind farm is then delivered to the onshore collection point by the transmission system. All the step-up transformers, HVDC ac/dc converters, compensators, and energy storage components, if any, are housed in an offshore substation.

The whole system basically consists of two parts. One is the wind energy generation and conversion system. Based on the operating mode, it is generally divided into two categories, fixed-speed and variable-speed systems.

The second is the transmission system. Offshore wind farms are usually located far from the onshore grid connection point. To transmit the power of the offshore wind farm from the offshore collection point to the onshore collection point, both HVAC and HVDC transmission links are available in practice, though with different features. HVAC is the simplest and most economic connection method when the distance between offshore and onshore connection points is less than $50 \mathrm{~km}$. HVDC, on the other hand, is dominating the market when the distance is above $50 \mathrm{~km}$ [4]. 


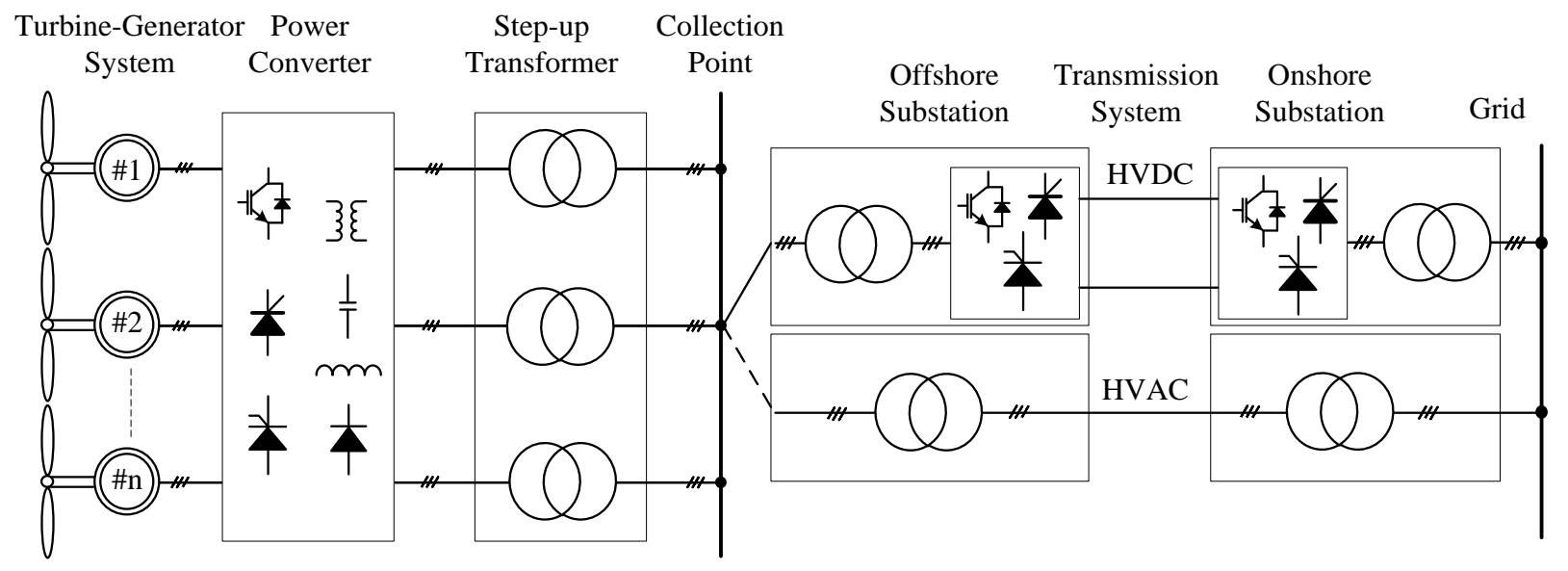

Figure 1-1 Basic structure of offshore wind farm [7], [8].

\subsection{Megawatt wind energy conversion system}

Each turbine-based system used in the offshore wind farm discussed in this dissertation is rated at megawatt levels. This is mainly propelled by the associated cost saving in initial foundation and the increase of energy harvest capability at higher altitudes with higher wind speeds. A variety of configurations have been proposed for megawatt WECS. They can be divided into two categories based on their operating mode, fixed-speed and variable-speed WECS [2].

\subsubsection{Fixed-speed WECS}

A typical configuration for a high-power fixed-speed WECS is shown in Figure 1-2. To assist the start-up of the turbine, a soft starter, a three-phase ac voltage controller, is used to limit the inrush current in the generator winding. Once the start-up process is over, the soft starter is bypassed by a switch, and the WECS is then connected to the grid through a transformer. To compensate for the inductive reactive power consumed by the induction generator, a capacitorbased power factor (PF) compensator is normally used. In practice, the compensator is composed of multiple capacitor banks, which can be switched into or out of the system individually to provide an optimal compensation according to the operating conditions of the generator.

Due to the use of a cost-effective and robust squirrel-cage induction generator (SCIG) with inexpensive soft starter, the fixed-speed WECS features simple structure, low cost, and reliable operation. However, compared to the variable-speed WECS, the fixed-speed system has lower energy conversion efficiency since it can achieve the maximum efficiency only at one given wind speed. 


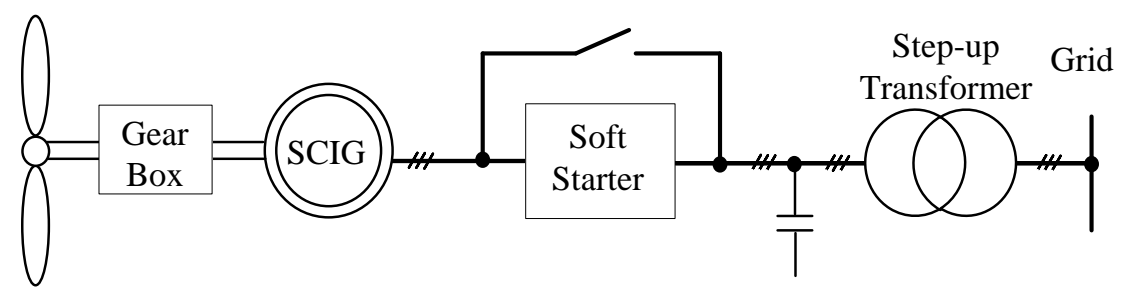

Figure 1-2 Fixed-speed WECS.

\subsubsection{Variable-speed WECS}

The variable-speed WECS can be divided into direct- and indirect-drive systems. The indirectdrive turbine requires a gearbox to match the low turbine speed to the high generator speed. The synchronous generator and induction generator equipped with full-capacity power converters have all been used in practical systems. In addition, doubly fed induction generators (DFIGs) with reduced-capacity converters and wound-rotor induction generators (WRIGs) with converter-controlled variable rotor resistance have also found practical applications. In directdrive turbines, low-speed synchronous generators with a large number of poles are employed. The speed of the synchronous generators is designed to match the turbine speed such that the gearbox normally required in the indirect-drive turbines is eliminated.

Figure 1-3 shows the typical configuration of a DFIG-based WECS. The stator is connected to the grid directly, whereas the rotor is connected to the grid via reduced-capacity power converters. The speed range of the DFIG-based WECS is around $\pm 30 \%$, which is $30 \%$ above and below the synchronous speed. The maximum slip determines the maximum power to be processed by the rotor circuit, which is around $30 \%$ of the rated power. The use of reducedcapacity converters results in reduction in cost, weight, and size. Compared with the fixed-speed system, the energy conversion efficiency of the DFIG-based system is greatly enhanced. However, the DFIG-based system has certain disadvantages that can not be neglected when considering for offshore applications. First, the connection of the power converter necessitates the use of a wound rotor with slip rings and brushes. The average life cycle of the brushes used in DFIG is only 6-12 months, which makes regular maintenance a required task. This drawback prevents DFIG from offshore applications where the maintenance cost is quite expensive. Second, a costly gearbox used to provide a high gear-ratio for the wind turbine rotating speed to be transformed to the generator speed also brings issues of increased cost, reduced reliability, and necessary regular maintenance. 


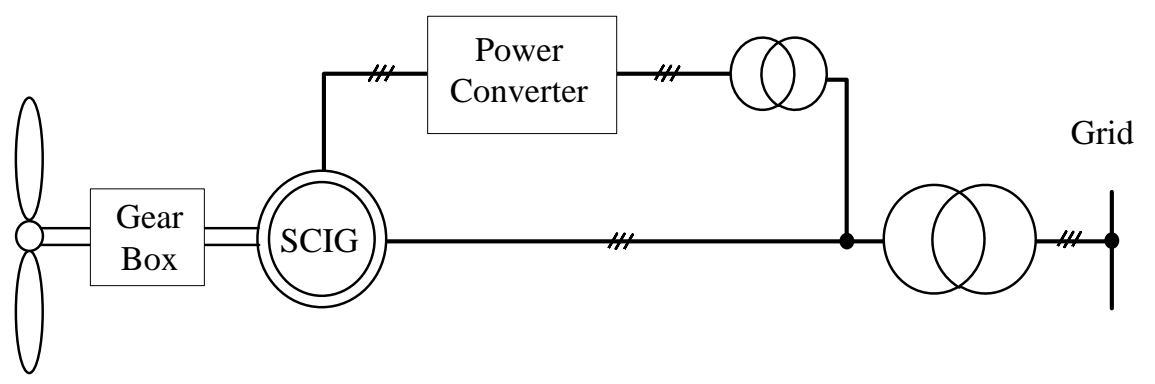

Figure 1-3 DFIG-based indirect-drive WECS.

Figure 1-4 shows the typical configuration of a direct-drive WECS where synchronous generators, including electrically excited synchronous generators (EESG) and permanent magnet synchronous generators (PMSG), are mostly used. All direct-drive configurations require a fullrating power converter to enable generator speed variations. Such a converter is costly and low in efficiency compared with the reduced-capacity converter in DFIG. However, the elimination of the gearbox offsets the increased cost. The full-rating power converter also brings full control capability which can be utilized to significantly improve fault ride-through capability of the generation system. Thus, the direct-drive configuration with a full-rating power converter becomes a favorable option for megawatt WECS.

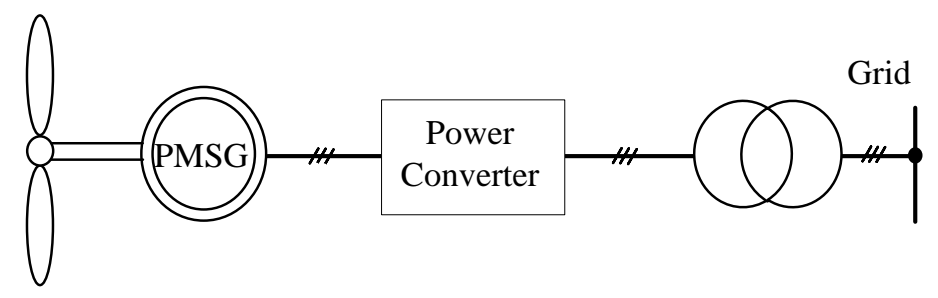

Figure 1-4 Direct-drive WECS.

\subsection{Transmission system}

Offshore wind farms are usually located far from the onshore grid connection point. To transmit the power of the offshore wind farm from the offshore collection point to the onshore collection point, both HVAC and HVDC transmission links are available.

\subsubsection{HVAC transmission system}

Figure 1-5 shows a typical HVAC system including an medium voltage alternate current (MVAC) collector system, an offshore substation, and an onshore substation possibly with the static VAR compensator (SVC). 
HVAC is the simplest and most economic connection method when the distance between offshore and onshore connection points is less than around $50 \mathrm{~km}$ [9]. However, as the distance goes up, HVAC has the following disadvantages [10], [11]. First, the submarine ac cable produces large amount of reactive current due to its high equivalent capacitance. As a result, the current-carrying capacity of the cable is significantly reduced with increasing transmission distances and voltage levels. Thus, reactive power compensations are required. Second, HVAC is unable to directly connect two ac power networks with different frequencies.

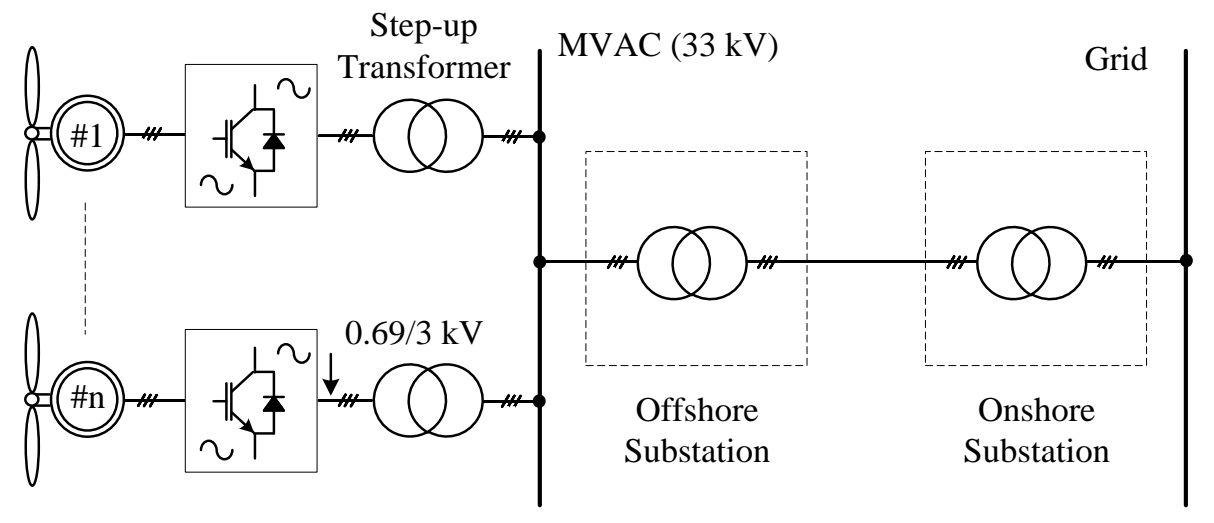

Figure 1-5 A typical HVAC system.

\subsubsection{HVDC transmission system}

With increasing transmission distances and voltage levels, HVDC transmission system is verified to be the most suitable option [12], [13]. Basically, there are conventional line commutated converter (LCC)-based HVDC system [14], [15], voltage source converter (VSC)based HVDC system [16], [17], and PWM CSC-based HVDC system [18] commissioned in the industry and/or reported in the literature.

(a) LCC-based HVDC system

Figure 1-6 shows the classical LCC-based HVDC system. LCC needs the grid voltage to assist the commutation of thyristor valves. Thus, it is susceptible to the grid disturbance and a relatively strong grid is required. The LCC-HVDC system needs large passive filters to reduce low-order harmonics and it cannot provide independent control of active and reactive powers [19], [20]. What is more, the footprint and weight of this system are huge. All these drawbacks make the LCC-based HVDC system less suitable for offshore applications even though it has been demonstrated for more than 50 years of service experience on land. 


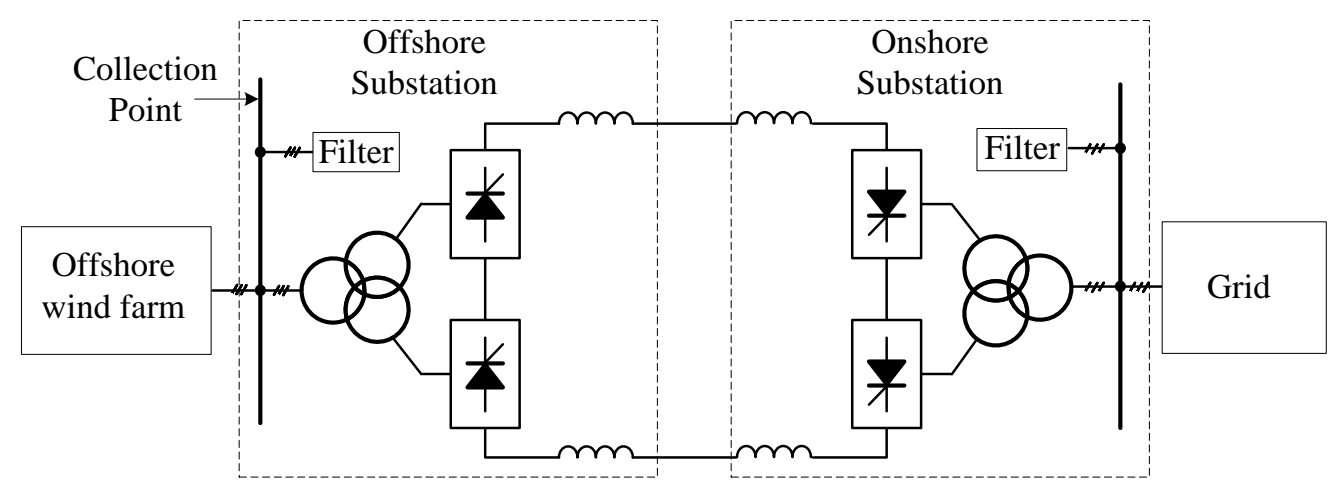

Figure 1-6 LCC-based HVDC system [14], [15].

(b) VSC-based HVDC system

VSC-based HVDC system in combination with offshore wind farm is already commissioned in practice, such as "HVDC Light" by ABB [7] and "HVDC Plus" by Siemens [8]. Figure 1-7 shows such a typical VSC-based HVDC system. The collected power of the wind farm is transferred from ac to dc by the wind farm-side VSCs and then transmitted to grid-side VSCs through the HVDC cables. The grid-side VSCs transmit available power to the grid and provide reactive power according to grid codes. The wind farm-side VSCs, step-up transformers, and other required components are housed in an offshore substation.

Compared with LCC-based HVDC system, VSC-based HVDC system offers a couple of advantages: self-commutated ability and independent control of active and reactive power. On the other hand, the cost and loss of VSC-based HVDC system are higher than that of LCC-based HVDC system. And, the reliability of VSC-based HVDC system is lower compared with LCCbased HVDC system due to the higher component count.

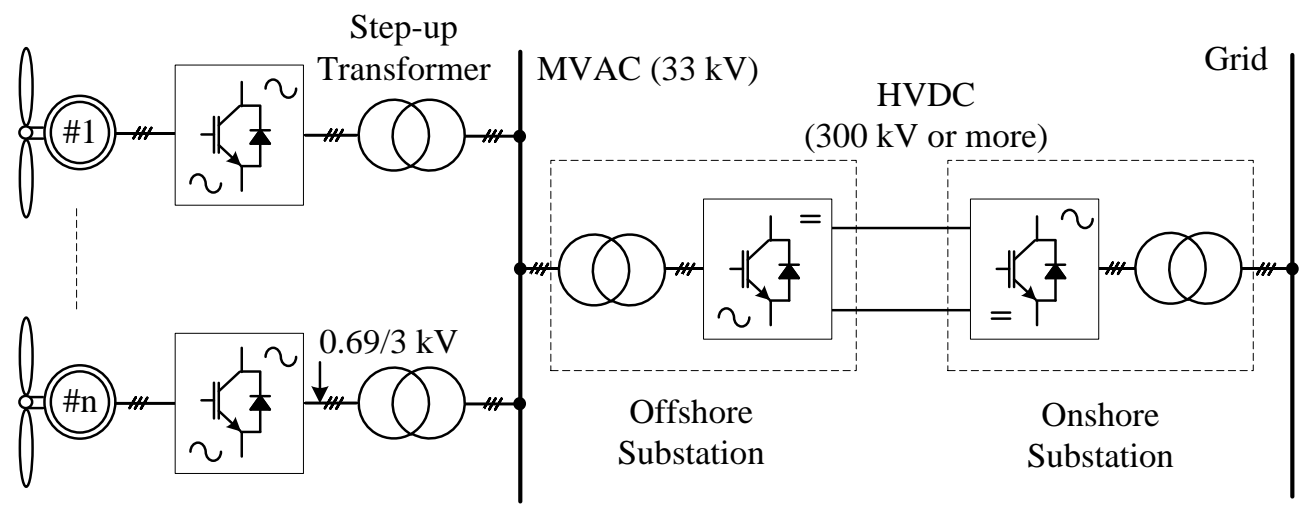

Figure 1-7 VSC-based HVDC system [16], [17]. 


\section{(c) PWM CSC-based HVDC system}

Recently, a PWM CSC-based HVDC system is proposed for offshore wind farms shown in Figure 1-8 [21]. A number of PWM CSCs are connected in series at both offshore and onshore sides, respectively. Compared with LCC-based HVDC system, PWM CSC-based one is based on self-commutated converters and has the ability of independent control of active and reactive power. Compared with the VSC-based HVDC system, it features a unique feature, which is the reliable short-circuit protection. To sum up, all these features make the PWM CSC-based configuration highly promising in offshore applications.

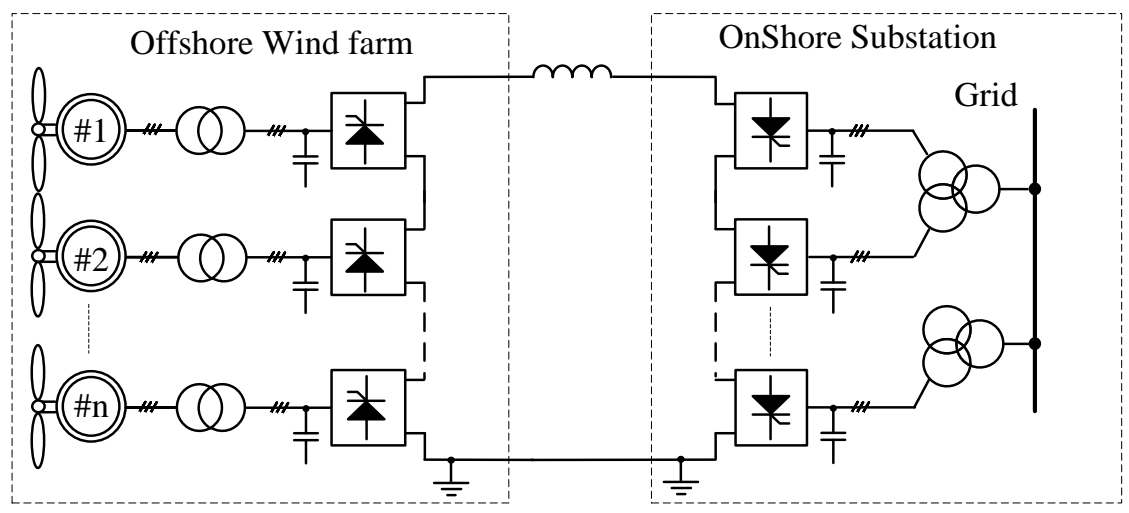

Figure 1-8 PWM CSC-based HVDC system [18].

\subsection{Offshore wind farm configuration}

Based on the connection manner of wind turbines, the offshore wind farm configurations proposed in literature and/or implemented in practice can be classified into two categories, parallel-connected and series-connected configurations [4], [23], and [24].

\subsubsection{Parallel-connected configuration}

Parallel connection is most widely used in present wind farms. In combination with the transmission system, the parallel-connected configuration can be further divided into three classes, parallel ac connection and HVAC system, parallel ac connection and HVDC system, and parallel dc connection and HVDC system.

Figure 1-5 shows the configuration of the parallel ac connection and HVAC system. The output voltage of each wind turbine is mainly $690 \mathrm{~V}$ (or 3000-4000 V), then stepped up to a medium voltage level by the step-up transformer. All the turbine systems are connected in 
parallel to form an MVAC collection system. The MVAC is then stepped up to an HVAC level $(60-245 \mathrm{kV})$ by another transformer housed in the offshore substation.

The second configuration is the parallel ac connection and HVDC system shown in Figure 1-7. This is the most popular interconnection approach for far located offshore wind farms with larger power capacity. The MVAC collection system is first converted to an HVAC level by a step-up transformer and then converted to an HVDC level by an ac/dc power converter. The step-up transformer and ac/dc converter are housed in an offshore substation. The receiving-end onshore substation contains a dc/ac converter and a step-down transformer, if any. Both CSC and VSC can be used in this configuration. Among all, MMC has become an important milestone in VSC HVDC-based offshore wind farms because of its enhanced reliability, near sinusoidal output waveform and high efficiency [25], [26].

Figure 1-9 shows the configuration of the parallel dc connection and HVDC system where an intermediate dc/dc converter is used to step the low output voltage (1200/5000 V) of the ac/dc converter up to an MVDC level (30-50 kV) [27], [28]. The output dc voltages of the dc/dc converters are then connected in parallel to form an MVDC collection system. The MVDC is then stepped up to an HVDC level using a central wind farm dc/dc converter housed in an offshore substation. The dc/dc converters consist of a dc/ac conversion stage, medium- or highfrequency transformer, and an ac/dc conversion stage [29], [30]. Compared with the step-up transformer and the ac/dc converter used in Figure 1-5 and Figure 1-7, the size and weight of the $\mathrm{dc} / \mathrm{dc}$ converter are smaller. Though this configuration is highly researched, it has not been implemented yet.

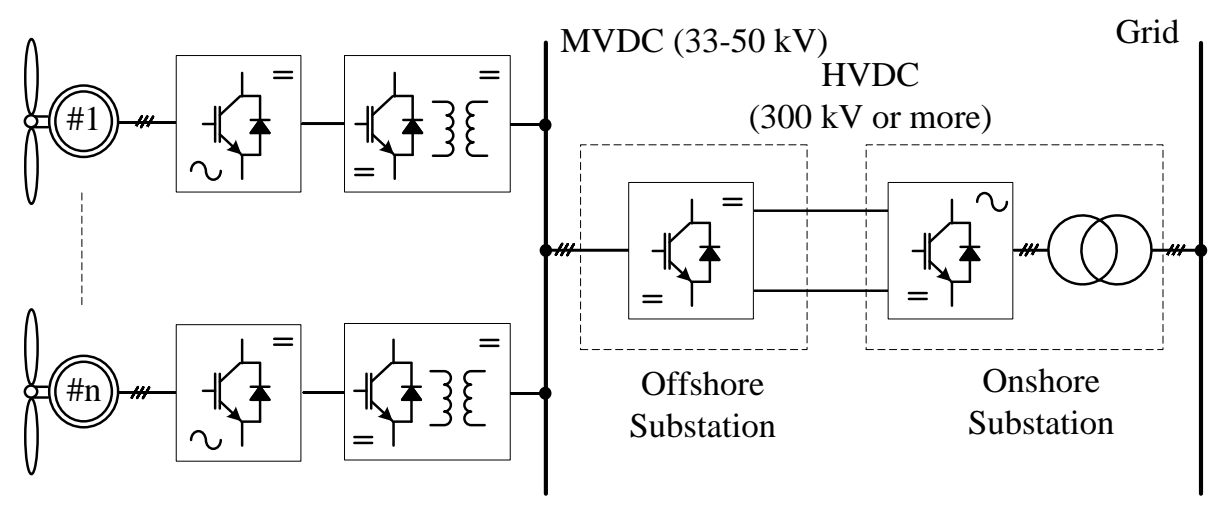

Figure 1-9 Parallel dc and HVDC system. 
In summary, all parallel-connected configurations need the offshore substation. Such an offshore substation is very costly and bulky and is the main disadvantage of the parallelconnected configuration.

\subsubsection{Series-connected configuration}

The series-connected configuration can eliminate the offshore substation, thus greatly lowering the cost of the whole system. Figure 1-10 shows such a series-connected configuration proposed for offshore wind farms. The dc output of each offshore converter is connected in series to reach an HVDC level so that the offshore substation can be eliminated.

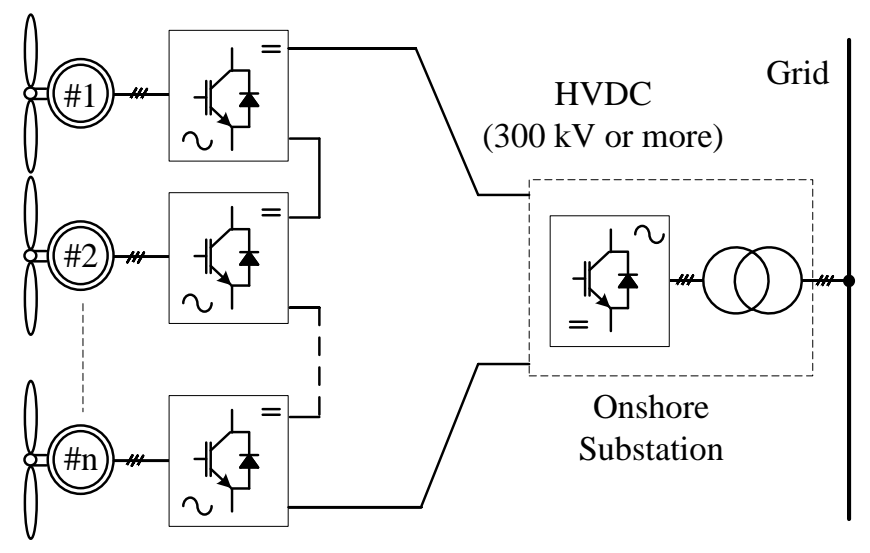

Figure 1-10 Series dc and HVDC system.

Various converters can be employed in this configuration. For example, a high-frequency converter-based configuration is proposed in [31]. Each turbine-side converter consists of a 3phase to 1-phase matrix converter, a high frequency transformer, and a single-phase ac-dc converter. However, such a high-frequency converter rated with an MW level is difficult to implement. Thyristor-based and PWM CSC-based configurations can be also found in [32], [18].

One main concern of the series-connected configurations is the generator insulation. The wind generator that is furthest from the grounding must be capable of withstanding a full transmission voltage. To tackle this problem, a three-phase low-frequency transformer is normally employed between the generator and the offshore converter as shown in Figure 1-8. Such a low-frequency high-power transformer, however, is heavy and bulky, adding additional burden to offshore construction because of the limited space either in the nacelle or in the tower of the turbine [6]. 


\subsection{Offshore substation}

As mentioned earlier, the offshore substation is used in existing offshore wind farms. However, there are a couple of constraints associated with the offshore substation that should be addressed considering the offshore conditions [34]. First, the employed components should be as compact as possible to reduce the overall size and weight. Second, the offshore environment is extremely harsh. Constant exposure to the salty air and water requires equipment to be located either indoors or in sealed enclosures. Third, the auxiliary supplies, such as generators or batteries, are required to ensure supply availability for the critical load during very low or no wind condition. Fourth, the maintenance access is limited and costly. Thus, the components should be maintenance-free with high reliability.

The offshore substation houses the offshore step-up transformers, HVDC converters, compensator, storage batteries, and other related components. Thus, it is very bulky and costly. Figure 1-11 shows one of the world's largest offshore substations used in the offshore wind farm "Dolwin2" which is commissioned by ABB [35]. The complete platform including substructure weighs around 23,000 tons and is around 100 meters long, 70 meters wide and 100 meters tall.

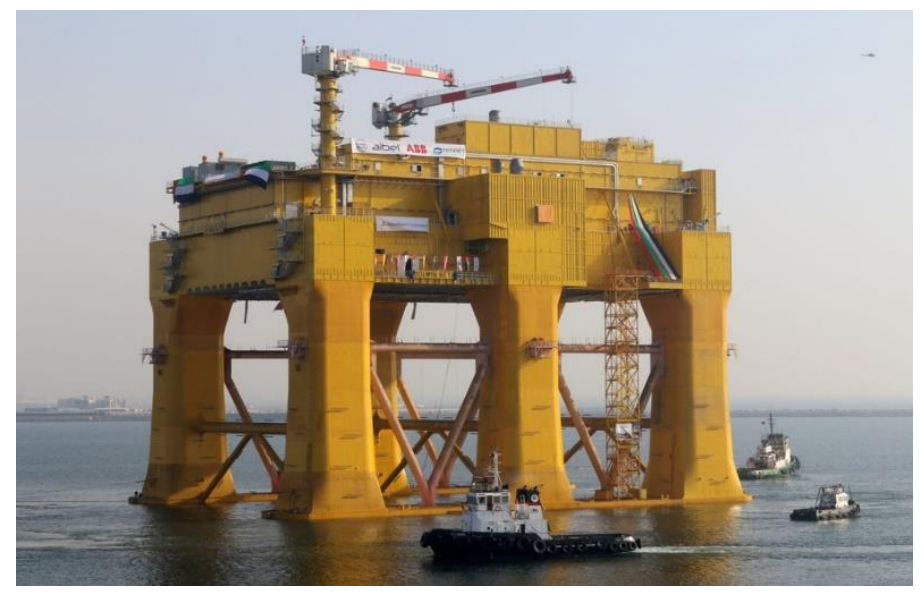

Figure 1-11 Offshore substation used in "Dolwin2” by ABB [35].

\subsection{Dissertation objectives}

\subsubsection{Thesis objectives}

As mentioned earlier, the PWM CSC-based series-connected configuration is a promising solution for offshore wind farms. But, a low-frequency high-power transformer which is heavy 
and bulky is needed for the issue of generator insulation. Therefore, this thesis endeavours to propose new configuration for CSC-based offshore wind farms with smaller size and weight. In addition, advanced modulation scheme with high dynamic performance, and optimized control schemes giving low cost and high reliability and efficiency will be proposed for the proposed configuration.

The main objectives of this dissertation are summarized as follows.

(1) New configuration with smaller size and weight for CSC-based offshore wind farm

The series-connected configuration can eliminate the offshore substation, but a three-phase low-frequency transformer is needed for the issue of generator insulation. Such a low-frequency transformer is heavy and bulky, introducing additional burden as the space in the nacelle or in the tower of the turbine is limited. Therefore, configuration with smaller size and weight is preferred for offshore wind farms.

(2) Advanced SVM scheme with superior low-order harmonics performance for CSCs

The switching frequency of high-power CSC is limited to a few hundred Hertz for reducing switching loss and device thermal stress. Under such a low switching frequency, conventional space vector modulation (SVM) featuring high dynamic performance cannot be used for CSC mainly due to its high-magnitude low-order harmonics. Thus, the second objective of the work is to propose an advanced SVM scheme with superior low-order harmonics performance.

(3) Equal power distribution among grid-side series-connected CSCs

Mismatches among series-connected CSCs exist and will introduce power imbalance. Power balancing among series-connected CSCs is an important consideration for system reliability. Otherwise, the system reliability may suffer. For example, the one that contributes a greater portion of the power will be thermally overstressed. Thus, another objective of the work is to ensure power balancing among the series-connected CSCs.

(4) Bipolar operation of CSC-based offshore wind farm

Bipolar operation features low insulation requirement, thus contributing to lower cost and higher reliability. However, a unique challenge exists for the CSC-based series-connected configuration operating under bipolar mode that is the dc-link current control. There are two 
paths for the dc-link current and the dc-link current control directly determines the efficiency of the bipolar system. Therefore, an optimal dc-link current control with high efficiency is needed.

(5) Optimal strategy for CSC-based offshore wind farm with low cost and high efficiency

First, the nominal number of onshore CSCs in previous study is simply set to be same as that of the offshore turbine units, introducing higher cost on onshore CSCs and higher operation losses under rated condition. Second, the wind speed at each turbine cannot be guaranteed to be identical, generating different dc-link reference currents. To ensure proper operation, the maximum one has to be selected to be the dc-link current of the wind farm. This lowers the system efficiency under derating conditions. Therefore, the last objective of the work is to propose an optimized strategy with low cost and high efficiency.

\subsubsection{Thesis outline}

This dissertation consists of seven chapters which are organized as follows.

In Chapter 1, the background and introduction of the study are presented.

In Chapter 2, new configurations are proposed for medium-voltage (MV) and low-voltage (LV) turbine-based series-connected offshore wind farms, respectively. Compared with existing configurations, the new ones are expected to be smaller in size and weight.

In Chapter 3, a natural sampling SVM (NS-SVM) scheme is proposed for grid-side CSCs. Compared with conventional SVM, NS-SVM features superior low-order harmonics performance without compromising other performance, such as low switching/sampling frequency and fast dynamic response. Besides, the optimal space vector sequence in terms of low-order harmonics performance based on NS-SVM is investigated and designed.

In Chapter 4, a power balancing control is proposed for the grid-side series-connected CSCs. The possible power imbalance among series-connected CSCs is analyzed and quantitatively defined. By applying the proposed power balancing scheme, both power balancing and other required control objectives are ensured simultaneously.

In Chapter 5, a bipolar CSC-based system is investigated and an optimal dc-link current control is proposed. Compared with monopolar operation, bipolar operation features lower insulation 
level, higher reliability, and larger power capacity. And compared with conventional dc-link current control, the proposed one gives higher efficiency.

In Chapter 6, an optimal strategy for the CSC-based series-connected offshore wind farm is proposed. The nominal number of onshore CSCs is optimized that cost saving on power converters is achieved and higher efficiency under rated condition is obtained. The efficiency of the wind farm under derating conditions is also improved by the optimized bypass operation of onshore CSCs.

The main contributions and conclusions of the dissertation are summarized in Chapter 7. Besides, possible future research work is also suggested. 


\section{Chapter 2}

\section{Medium-Frequency Transformer Based Offshore Wind Farm Configuration}

Apart from considering reliability and efficiency as the main requisites for all onshore systems, the footprints and weights of the components are particularly important for offshore systems. As illustrated in the previous chapter, existing configurations either need an offshore substation which is very bulky and costly or require a high-power low-frequency transformer which is very heavy. Therefore, the objective of this chapter is to propose new configuration for CSC-based offshore wind farm with smaller size and weight.

This chapter starts with an introduction of the proposed new configurations, medium-voltage (MV) and low-voltage (LV) turbine-based configurations, followed by the illustration of features of these configurations. To ensure safety operation, voltage balance control is proposed for MV turbine-based configuration, while current balance control is proposed for LV turbine-based one. Both simulation and experimental results are provided to verify the performance of the proposed configurations and control schemes.

\subsection{Medium-frequency transformer based configuration}

Figure 2-1 shows the overall structure of the CSC-based series-connected offshore wind farm [36]. In the offshore part, either MV (3-4 kV) or LV (690 V) wind turbine can be used. Accordingly, different generator converters are needed for MV and LV systems, respectively. The output of each generator converter is connected in series to reach an HVDC level. The captured power of the wind farm is transferred to the onshore grid system by undersea cables. In the onshore part, a number of CSCs are connected in series to form a centralized dc/ac converter. The centralized dc/ac converter is then connected to the grid system through multi-winding transformers. One common dc-link inductor $L_{d c}$ is shared by offshore and onshore converters. 


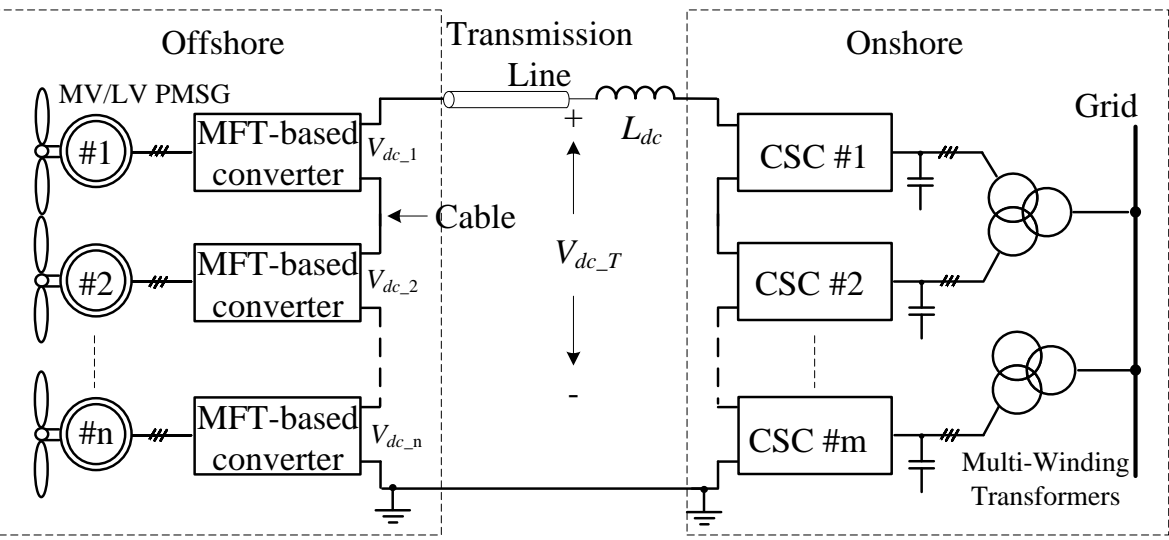

Figure 2-1 CSC-based series-connected offshore wind farm.

\subsubsection{Configuration with medium-voltage turbine}

Figure 2-2 shows the topology of a single MV-based system. It consists of an MV PMSG, a three-phase diode rectifier, a modular medium-frequency transformer (MFT)-based converter, and a current source converter (CSC). The MFT-based converter is composed of a number of $\mathrm{H}$ bridge converters with series input and output.

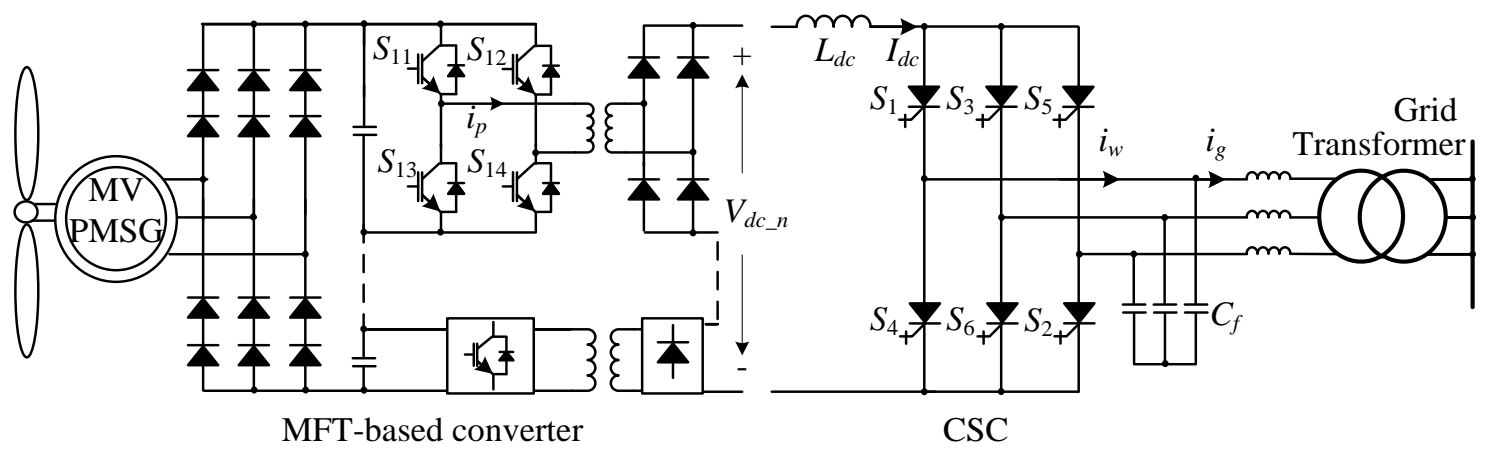

Figure 2-2 MV turbine-based configuration.

The three-phase diode rectifier interfacing the generator shown in Figure 2-2 displays the advantages of high reliability, low cost, and small size and weight. The side effect of this passive converter is that it generates a relatively high torque ripple in the generator. However, various methods have been proposed in literature to solve this problem [37]. Furthermore, the synchronous inductance of a PMSG is usually above 0.4 per unit (pu) for high-power, low-speed wind applications [38] which helps reduce the torque ripple further [22].

The modular MFT-based dc-dc converter plays two roles. First, it is for generator-side control. The primary objective for the generator-side converter is to obtain the maximum power input 
from varying wind speeds. This can be achieved by regulating the modular MFT-based converter. Second, the MFT is used to solve the issue of generator insulation. In a series-connected offshore wind farm, the wind generator that is farthest from the grounding point must be capable of withstanding the full transmission voltage. Compared with the bulky low-frequency transformer [40], the MFT is smaller in size and weight which is particularly important for offshore applications. Furthermore, a modular design is implemented based on a number of cells that are connected in series at both input and output. This helps reduce the burden of transformer implementation as one transformer carries only one part of a megawatt-level power. The modular design of the dc-dc converter also contributes to the choice of low-cost, low-voltage switching devices instead of high-voltage ones.

The nominal number of H-bridge converters is determined by: (a) input dc voltage, (b) the voltage rating of the selected insulated-gate bipolar transistor (IGBT), and (c) the chosen cell voltage. The rated input dc voltage is approximately $5000 \mathrm{~V}$ for a $4000 \mathrm{~V}$ PMSG-based system. $1700 \mathrm{~V}$ IGBT is selected because it is the most suitable switching device in terms of cost, voltage utilization, and failure in time rate for MV applications [41]. Given a cell voltage of $1000 \mathrm{~V}$, five cells (without redundancy) needs be connected in series at both input and output. A dc-dc converter with six cells is normally used in practice for redundancy purpose $(\mathrm{N}+1)$.

\subsubsection{Configuration with low-voltage turbine}

Figure 2-3 shows the topology of a single LV turbine-based system. Compared with the MV configuration shown in Figure 2-2, the LV one has a different offshore converter. The offshore converter is also a modular MFT-based converter, but with a parallel connection at input and a series connection at output.

Apart from regulating the generator speed to achieve maximum power point tracking (MPPT) and solving the issue of generator insulation, the modular MFTs-based converter used in the LV configuration plays an additional role that is to step up the low input voltage to a medium voltage level to be compatible with the use of MV CSC. The number of modules of the modular MFTbased converter is determined by the rating of the MV CSC. For example, for a $690 \mathrm{~V}, 1 \mathrm{MW}$ WECS, six modules (turn ratio of each MFT is 1:1) are needed for a $4160 \mathrm{~V}$ CSC where the dclink current and voltage (average value) are around $220 \mathrm{~A}$ and $4500 \mathrm{~V}$. 


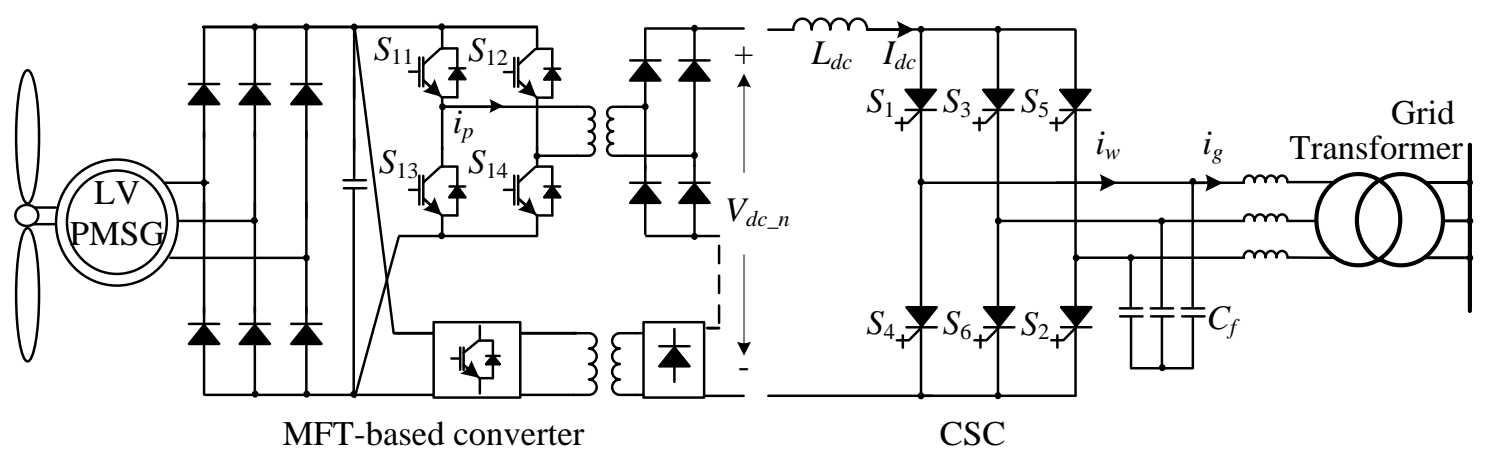

Figure 2-3 Proposed LV turbine-based configuration.

\subsubsection{Medium-frequency transformer}

Medium-frequency transformer with smaller size and weight is a good solution for applications where size and weight restrictions are becoming serious and demanding. For example, in the electric traction applications, the size and weight of the on-board components are a major constraint facing train manufactures because reducing the amount of space used by equipment means that more space is available for passengers. The low-frequency traction transformer (15 $\mathrm{kV} / 16.7 \mathrm{~Hz}$ or $25 \mathrm{kV} / 50 \mathrm{~Hz}$ ), is one of heavy pieces of equipment on a train. $\mathrm{ABB}$ has commissioned a power electronic traction transformer system where the traditional lowfrequency transformer is replaced with MFTs as shown in Figure 2-4 [42], [43]. The size and weight of the system is reduced without compromising performance. It has been installed on the locomotive and has been in service officially since 2012 .

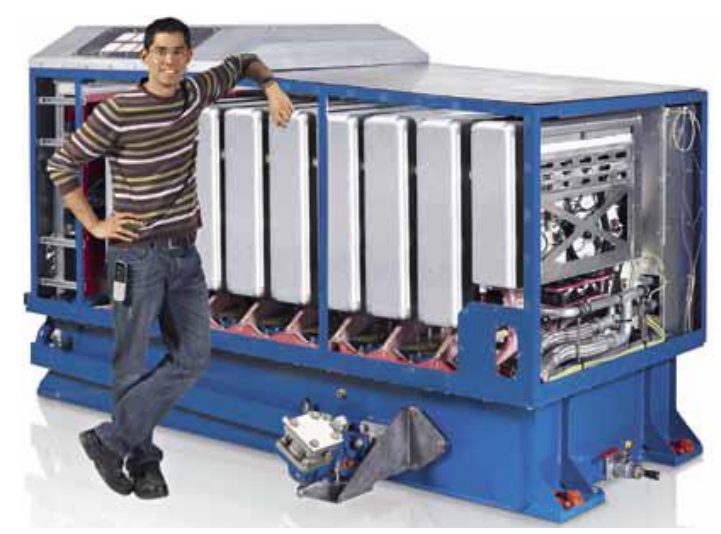

Figure 2-4 Power-electronic traction transformer system by ABB [42], [43].

The system is modular that consists of 8 power converters ( 9 for redundancy), 8 MFTs, and other related components. The maximum power rating of this system is 1.8 MVA with each MFT being around $225 \mathrm{kVA}$. The frequency used is $1.8 \mathrm{kHz}$. Each MFT is built around a 
nanocrystalline $\mathrm{C}$-cut core. This magnetic material is retained due to the low loss property at increased frequencies and relatively high saturation induction (around $1.2 \mathrm{~T}$ ). The nanocrystalline material is preferred over the cheaper amorphous material (which also exhibits higher saturation induction in the range of $1.5 \mathrm{~T}$ ) mainly because of the noise issue and the losses [43].

As mentioned earlier, compared with all onshore applications, the footprint and weight are particularly important for offshore applications. In addition, the space either in the nacelle or in the tower of the turbine is limited. Then the offshore system will greatly benefit from MFT which is smaller in size and weight compared with traditional low-frequency transformers. The design of MFT which can completely follow the one commissioned by ABB and is not the focus of the thesis.

In practice, the following two items should be considered for the implementation of the MFT. One is the operating frequency. Increasing operating frequency results in a large reduction in size and weight of the transformer. However, in megawatts-level wind energy systems, it is not the case. A significant challenge will be introduced to the thermal and cooling system design due to the combination of small size and high losses as increasing operation frequency. An important finding is that above a certain frequency, there is hardly any size reduction when increasing the frequency further [44]. Therefore, in practice, an optimum design should be a trade-off between operation frequency and size to achieve a best overall performance. In the present study, a switching frequency of $1200 \mathrm{~Hz}$ is employed.

The second challenge comes from the insulation design of high-power MFT. In the proposed series-connected configuration, MFTs are used for the issue of generator insulation. However, the technology of high-power MFTs with high insulation level is not mature. Based on the search of the literature and industry, there are a single $1 \mathrm{MVA} / 20 \mathrm{kHz}$ transformer with a $300 \mathrm{kV}$ insulation requirement [46], and a modular 1.8 MVA/1.8 kHz transformer (225 kVA for each transformer) with $75 \mathrm{kV}$ insulation used for traction by ABB [42], [43]. Therefore, a maximum transmission level below $300 \mathrm{kV}$ is promising for the proposed modular MFTs (1 MVA/1.2 kHz) (166 kVA for each transformer). On the other hand, it is more reasonable setting the transmission voltage at a medium voltage level, around $100 \mathrm{kV}$, for example, based on the search of the industry [42], [43]. 


\subsection{Control of MFT-based converter}

The control system of the series-connected offshore wind farm consists of two parts [40]. One is the control of offshore converters aiming to achieve MPPT. The other is the control of onshore converters consisting of dc-link current control and reactive power control. The control of onshore and offshore converters is decoupled. In this section, the control of offshore converters will be discussed.

The constituent modules of the modular converter in both MV and LV configurations are designed to be identical. However, they cannot be guaranteed to be identical mainly due to manufacturing techniques. The mismatch of components will lead to uneven distribution of voltage/current among the constituent modules. Thus, additional control scheme is needed to ensure equal power distribution among all the modules. In this section, an input capacitor voltage balance control is proposed for MV configuration, while a current balancing control is proposed for LV configuration.

\subsubsection{Voltage balance control for MV configuration}

The constituent modules of the modular converter (Figure 2-2) are designed to be identical. Assuming that all the involved components of the five constituent modules are identical, then the captured wind power will be evenly distributed among them. Then, an equal voltage distribution of input capacitors among the constituent modules will be achieved as well. The modular converter therefore can be simplified to one H-bridge converter.

However, given existing manufacturing techniques, the components used may not display exactly same characteristics. For example, the turns ratio of MFTs may be slightly mismatched, $1: k 1 \neq 1: k 2 \neq \ldots 1: k 5$. As a result, the operation of the modular converter is destabilized if only a common duty ratio alone is employed [47], [48]. The module with the lowest turn ratio has the highest input capacitor voltage and constitutes the largest proportion of total power. The voltage imbalance causes overvoltage, which may either destroy the switching devices or induce unwanted protection circuit actuation.

In summary, two control objectives must be ensured. One is MPPT. The other is voltage balance control of the modular MFT-based converter.

a) Voltage balancing control 
Figure 2-5 shows the control scheme of MV configuration. Note that MPPT is mature in practice and can be found in related research [38], thus not repeated here. The output of MPPT is the duty cycle $d_{\text {com }}$ of the dc-dc converter. MPPT is achieved upon applying the common duty cycle $d_{\text {com }}$ to the dc-dc converter. For example, when the amount of captured wind power increases with wind speed, the duty cycle $d_{c o m}$ increase as well.

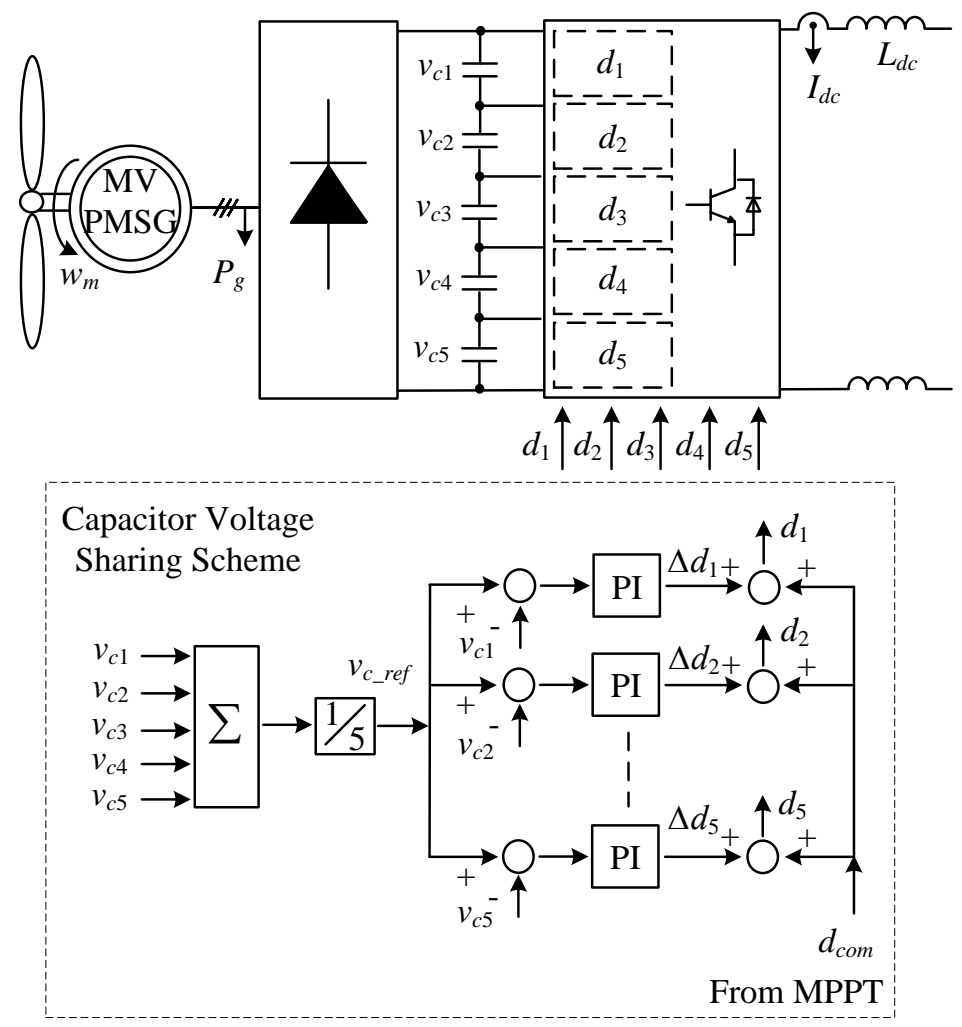

Figure 2-5 Voltage balance control for MV configuration.

The proposed capacitor voltage balancing scheme adjusts the duty cycle of each H-bridge converter to achieve an even distribution of input capacitor voltages. The applied duty cycles $d_{1}$, $d_{2} \ldots$, and $d_{5}$, are composed of two parts: one is the common duty cycle $d_{c o m}$ that represents MPPT, and the other is the individual part $\Delta d_{1}, \Delta d_{2} \ldots$, and $\Delta d_{5}$ that originates from the regulation of input capacitor voltage balancing control. With control under the common duty cycle $d_{\text {com }}$, the module with a larger turn ratio tends to draw higher input current than other modules do. This occurrence is impossible under steady state because the series connection at the input mandates that the input currents of the constituent modules should be equal. Hence, the capacitor of the module with a larger turn ratio must discharge more to compensate for the required current; this lower the capacitor voltage more in this module than in others. Therefore, 
input voltage loops are established to regulate individual input capacitor voltages by using classical PI controllers. The regulator output is obtained by adjusting the duty cycles required for voltage balancing. In contrast to the common duty cycle $d_{\text {com }}$, the module with a higher input capacitor voltage has a higher duty cycle with $d_{c o m}+\Delta d$. In the process, additional power is transferred to the load that more power is discharged from the capacitor. The discharge reduces the capacitor voltage, and the regulation continues until voltages are balanced.

b) Stability analysis of the voltage balance control

Figure 2-6 shows the simplified model used to analyze the stability of the voltage balance control shown in Figure 2-6. Each converter is modeled as an equivalent resistance, $R_{\text {neg }}$ [39], connected to its input capacitor. The regulation process is modeled as an ideal current source, $i_{\text {reg }}$ $=k_{v}\left(v_{c_{-} r e f}-v_{c N}\right), N=1,2, \ldots, 5$, connected across the input voltage of each converter. $k_{v}$ is the gain of the voltage balancing controller.

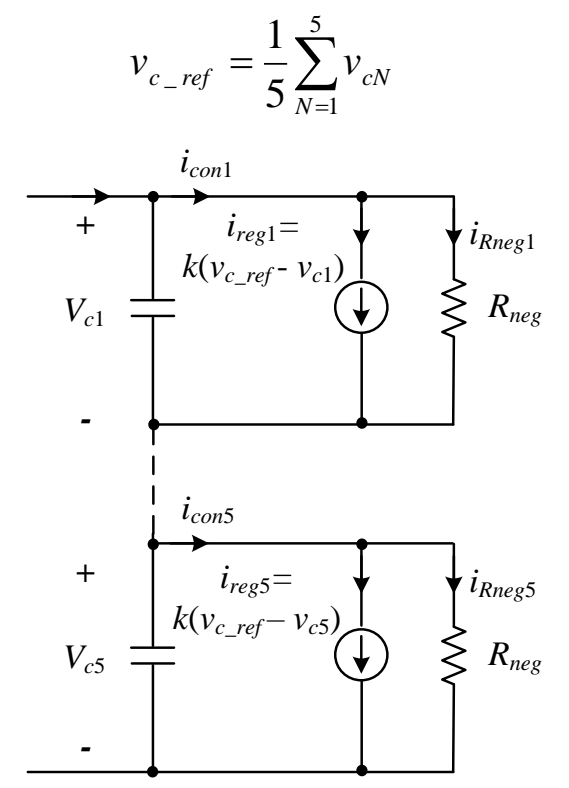

Figure 2-6 Equivalent circuit used for stability analysis.

The objective is to ensure an input voltage balancing among all the converters. The condition for the system stability shown in Figure 2-5, is that for an increase in any converter input voltage, the input current drawn by the converter should increase as well. This ensures that the higher input current discharges appropriately to reduce the input voltage. 
In terms of ac perturbation quantities, the condition for stability is that ${\hat{v_{c}}}_{r_{-} e f}-\hat{v}_{c N}$ should be in phase with $\hat{i}_{\text {ave }}^{\wedge}-i_{\text {conN }}^{\wedge} \cdot i_{\text {ave }}$ is the average current of all the converters.

$$
i_{\text {ave }}=\frac{1}{5} \sum_{N=1}^{5} i_{c o n}
$$

From Figure 2-6

$$
\begin{aligned}
& {\hat{i_{c o n} 1}}^{\wedge}=k_{v}\left(\hat{v}_{c_{-} r e f}^{\wedge}-\hat{v_{c 1}}\right)+\frac{\hat{v_{c 1}}}{R_{n e g}} \\
& i_{\text {con } 5}^{\wedge}=k_{v}\left(\hat{v}_{c_{-} r e f}-\hat{v_{c 5}}\right)+\frac{\hat{v_{c 5}}}{R_{n e g}}
\end{aligned}
$$

Then $\hat{i_{\text {ave }}}$ is derived based on (2-3)

$$
i_{\text {ave }}^{\wedge}=\frac{1}{5} \sum_{N=1}^{5} i_{\text {con } 1}^{\wedge}=\frac{v_{c_{-} r e f}^{\wedge}}{R_{n e g}}
$$

Combining (2-3) and (2-4) gives

$$
\hat{i_{\text {ave }}}-\hat{i}_{\text {con } 1}^{\wedge}=\left(\frac{1}{R_{\text {neg }}}-k_{v}\right)\left(\hat{v}_{c_{-} r e f}^{\wedge}-\hat{v_{c 1}}\right)
$$

As mentioned earlier, $\left(\frac{1}{R_{n e g}}-k_{v}\right)$ should be larger than zero to ensure a stable operation, and this is the basic guideline for the design of $k_{v}$.

\subsubsection{Current balance control for $\mathrm{LV}$ configuration}

In contrast with MV configuration where a voltage balance control is needed, an average current balance control is required to ensure even current distribution among the constituent modules of the modular MFT-based converter.

Figure 2-7 illustrates the proposed average current balance control. The applied duty cycles $d_{1}$, $d_{2} \ldots$, and $d_{6}$ are composed of two parts: one is the common duty cycle $d_{c o m}$ that represents MPPT, and the other are the individual parts $\Delta d_{1}, \Delta d_{2} \ldots$, and $\Delta d_{6}$ that originate from the average 
current balancing regulator. With control under the common duty cycle $d_{c o m}$, the module with a larger turn ratio tends to draw higher input current (power) than other modules do. Hence, the module with a larger turn ratio must transfer less power to the load to reduce its input average current. Therefore, input average current loops are established to regulate individual input average current by using classical PI controllers. The regulator output is obtained by adjusting the duty cycles required for current balancing. In contrast to the common duty cycle $d_{c o m}$, the module with a higher input average current has a lower duty cycle with $d_{c o m}-\Delta d$; this lower duty cycle reduces the power transferred to the load and thus its input average current decreases. This regulation continues until input average currents of the constituent modules are balanced.

The stability analysis of the current balancing control shown in Figure 2-7 is similar with that of the voltage balancing control shown in Figure 2-5, thus not repeated here.
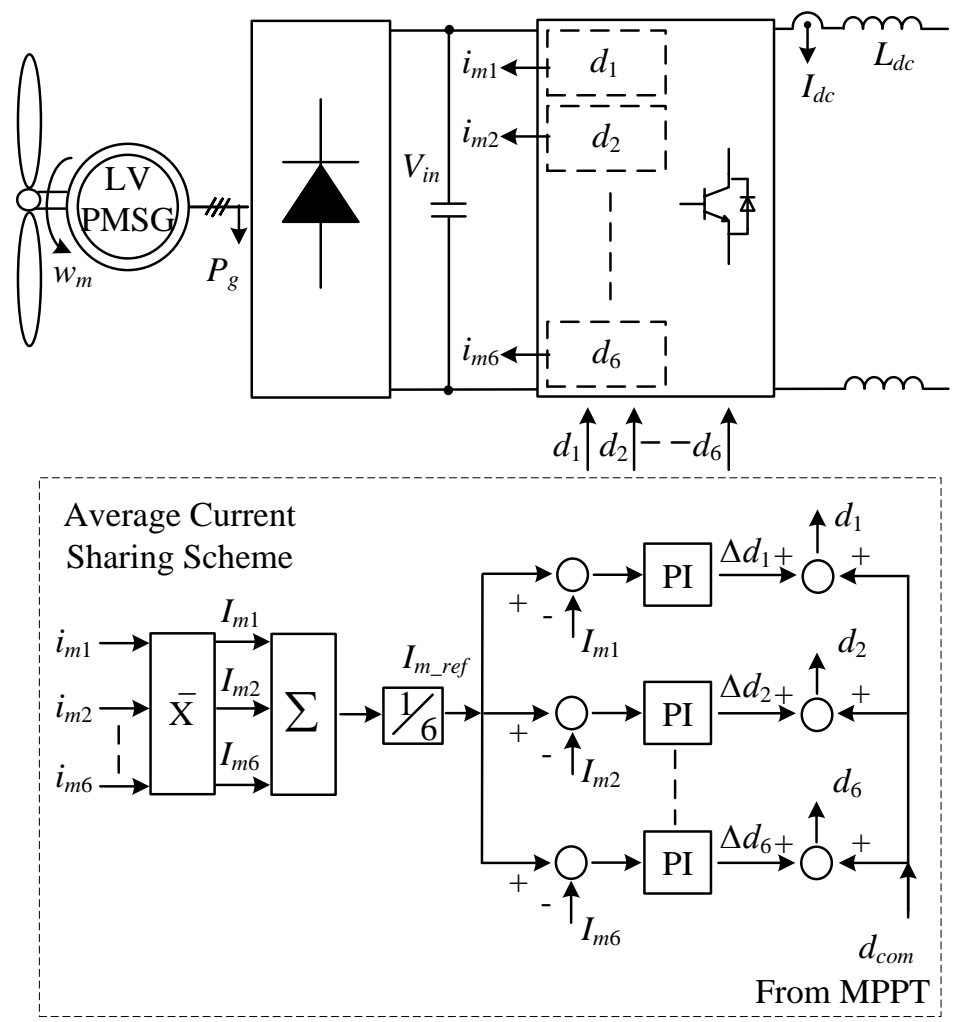

Figure 2-7 Average current balance control for LV configuration.

\subsection{DC-link voltage investigation}

In CSC-based applications, MV drives [22], for example, the dc-link current used for control is mainly considered, while the dc-link voltage relating to the system insulation is determined by 
the system rating and thus does not need to be paid additional attention. In the series-connected offshore wind farm configuration, however, it is not the case. The dc-link voltage is determined by both the system rating and the cables between offshore converters and between offshore and onshore converters. In this section, two items are investigated when considering the effect of cables, one is dc-link voltage which determines the system insulation and cost, and the other is dc-link current which affects the performance of control objectives.

\subsubsection{Offshore converter}

The offshore modular converter used in the MV turbine unit is a series-input series-output converter as shown in Figure 2-2, and it is a parallel-input series-output converter for the LV turbine unit as shown in Figure 2-3. Seeing from the output of each converter, the two types of converters are same that can both be replaced with a pulse-wave voltage source as shown in Figure 2-8 where the conventional phase-shifted modulation is used.

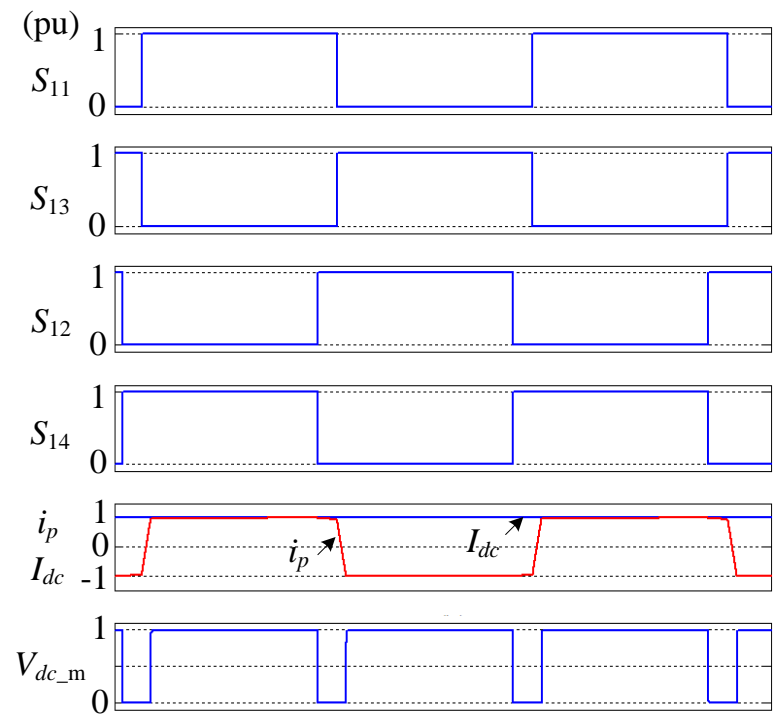

Figure 2-8 Key waveforms of each offshore converter.

In practice, the wind speed at each wind turbine cannot be guaranteed to be identical, which means the magnitudes of the output voltages $\left(V_{d c_{-}}\right)$of offshore converters are possibly different. In addition, each offshore generator unit is controlled individually, which means the initial phase angle of $V_{d c_{-} \mathrm{n}}$ is possibly different. Therefore, the pulse-wave voltage of each offshore converter can be expressed as 


$$
v_{d c_{-} n}(t)=d_{n}+a_{n_{-} m} \sum_{m=1}^{\infty} \cos \left\{2 \pi m f\left(t+\Delta t_{n}\right)\right\}
$$

where $d_{n}$ is the duty cycle of $v_{d c_{-} \mathrm{n}}$ for unit \#n, $a_{n_{-} m}\left(0 \leq a_{n_{-} \mathrm{m}} \leq 1\right)$ is the magnitude coefficient of the m-order component of $v_{d c_{-} \mathrm{n}}$, and $\Delta t_{\mathrm{n}}$ represents the initial phase angle of $v_{d c_{-} \mathrm{n}}$. Note that $\Delta t_{\mathrm{n}}$ could be any possible numbers, while it can be modified to fall into the range of $0 \leq \Delta t_{\mathrm{n}} \leq 1 / f$ where $f$ is the fundamental frequency, that is $2400 \mathrm{~Hz}$.

For example, the time domain and frequency domain waveforms of $v_{d c_{-} \mathrm{n}}$ under $d=0.9$ are illustrated in Figure 2-9.

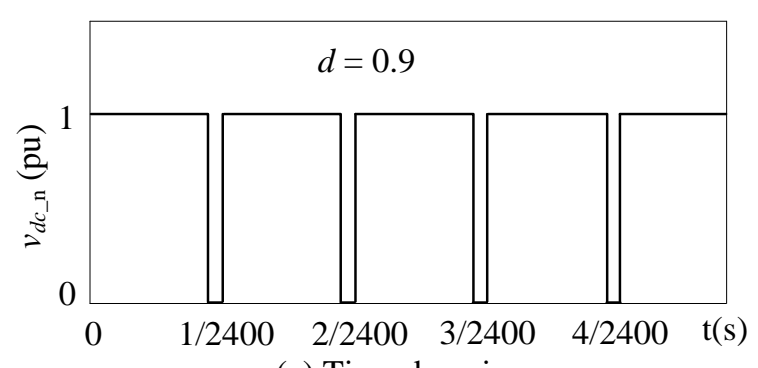

(a) Time domain

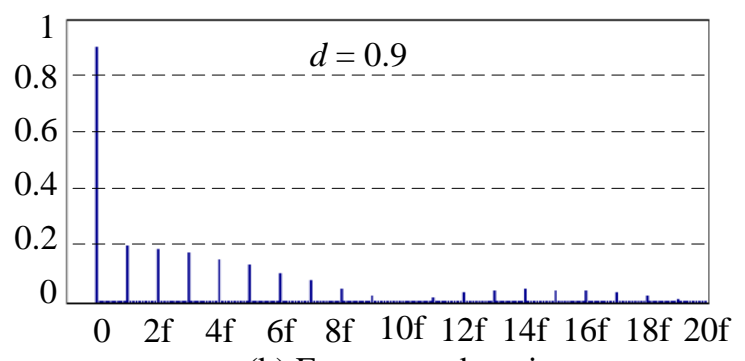

(b) Frequency domain

Figure 2-9 Pulse-wave voltage source. (a) time domain. (b) frequency domain.

A number of such pulse-wave voltages is connected in series to reach an HVDC level. However, there are cables connected between offshore converters and cables between offshore and onshore converters as shown in Figure 2-1. Then, the dc-link voltage cannot be simply thought to be equal to the sum of these pulse-wave voltages. Instead, the voltage reflection at the end of the cables possibly increases the peak value of these pulse-wave voltages, leading to higher dc-link voltage. Also, the performance of the dc-link current under the above condition needs to be evaluated as it affects the performance of control objectives of the system.

\subsubsection{Cable modeling}

As shown in Figure 2-10, single-core submarine cables are used between series-connected offshore converters and between offshore and onshore converters [49]. The key parameters of the cables, such as the length, the capacity, the insulation level, and other parameters like capacitance, inductance, and resistance, depend on a couple of factors: the configuration of the offshore wind farm, height of the wind turbine, system rating, and so on. Here, in the present work, the length of the cable between two adjacent offshore converters is set to $1 \mathrm{~km}$, while the 
one between offshore and onshore converters is set to $50 \mathrm{~km}$. All the related parameters are listed in Table A-1.

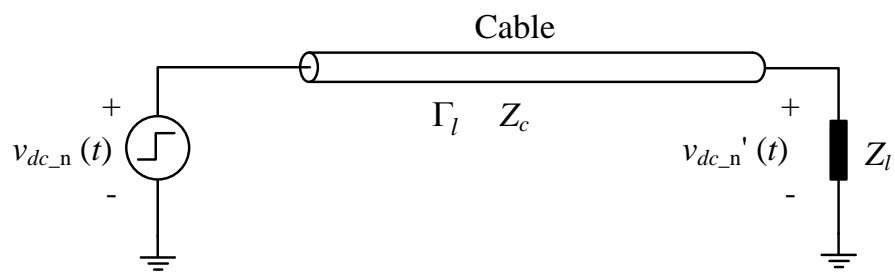

Figure 2-10 Configuration of a cable.

Figure 2-10 shows such a configuration where $Z_{l}$ is the impedance of the load and $Z_{c}$ is the characteristic impedance of the cable. One important feature of the distributed-circuit cable is the possible voltage reflection at the end of the cable. The voltage reflection coefficient $\Gamma_{l}$, is defined as $[50]$

$$
\Gamma_{l}=\frac{Z_{l}-Z_{c}}{Z_{l}+Z_{c}}
$$

Considering a lossless cable, the characteristic impedance of the cable is defined as [50]

$$
Z_{c}=\sqrt{\frac{L_{\text {cable }}}{C_{\text {cable }}}}
$$

where $L_{\text {cable }}$ is the inductance per unit length and $C_{\text {cable }}$ is the capacitance per unit length of the cable.

The typical characteristics of the voltage reflection coefficient are [50]

(a) If $Z_{l}=\infty$ (open circuit) $\Gamma_{l}=1$, which means the peak value at the end of the cable will be doubled,

(b) If $Z_{l}=0$ (short circuit) $\Gamma_{l}=-1$, and

(c) If $Z_{l}=Z_{c}$ (matched impedance) $\Gamma_{l}=0$. In this case, there is no voltage reflection in the end of the cable that the voltages at both ends are same in magnitude.

From the frequency domain perspective, the single-core distributed parameter cable can be approximately modeled by cascading a couple of identical RLC sections shown in Figure 2-11, 
where the resistance, inductance, and capacitance are uniformly distributed along the cable.

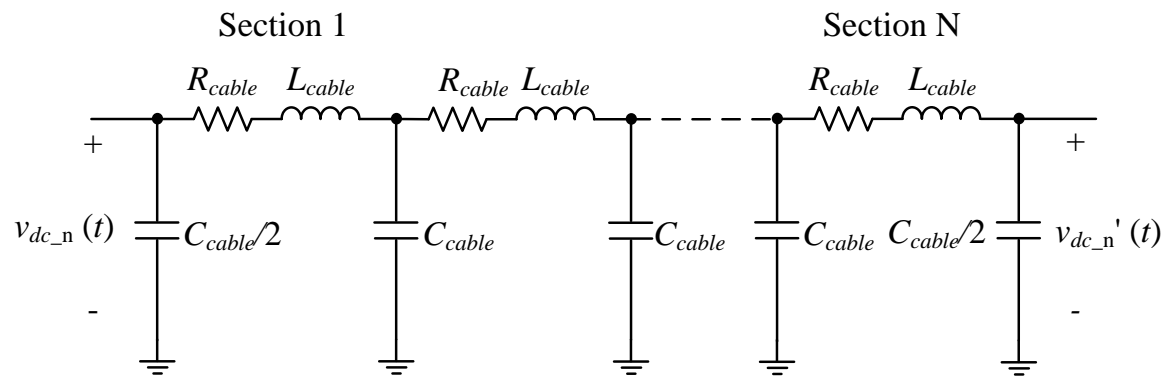

Figure 2-11 LRC sections-based single-core cable.

First, unlike a real distributed model which has an infinite number of states, the RLC section linear model has a finite number of states that enables the computation of a linear state-space model. The number of sections to be used depends on the frequency range to be represented. An approximation of the maximum frequency range represented by the RLC line model is given by the following equation [51]

$$
f_{\max }=\frac{N v}{8 l_{c}}
$$

where $\mathrm{N}$ is the number of RLC sections, $l_{c}$ is the cable length, and $v$ is the propagation speed

$$
v=\frac{1}{\sqrt{L_{\text {cable }} C_{\text {cable }}}}
$$

For example, for a $1 \mathrm{~km}$ cable having a propagation speed of around $70,000 \mathrm{~km} / \mathrm{s}$, the maximum frequency range represented with a single RLC section is approximately $8.75 \mathrm{kHz}$, while the number for two RLC sections is $17.5 \mathrm{kHz}$.

The transfer function of one RLC section is expressed as:

$$
\frac{V_{d c_{-} n}{ }^{\prime}(s)}{V_{d c_{-} n}(s)}=\frac{1}{\frac{L_{\text {cable }} C_{\text {cable }}}{2} s^{2}+\frac{R_{\text {cable }} C_{\text {cable }}}{2} s+1}
$$

The resultant magnitude response is illustrated in Figure 2-12. The cutoff frequency for onesection model is around $20 \mathrm{kHz}$, and is increasing as increased number of RLC sections. In other words, a model with more RLC sections can present higher frequency and is more likely a real distributed-circuit model. Shown in Figure 2-12, the main harmonics are located at below $48 \mathrm{kHz}$, 
which means at least 5 RLC sections are needed to mode a $1 \mathrm{~km}$ cable. Similarly, the $50 \mathrm{~km}$ cable connected between offshore and onshore converters needs at least 250 RLC sections to represent a frequency of $48 \mathrm{kHz}$.

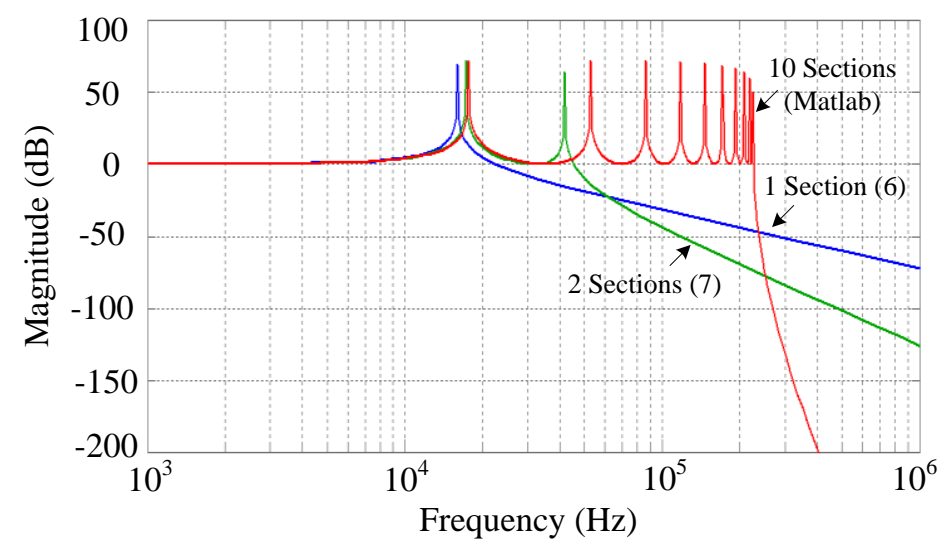

Figure 2-12 Magnitude response of the transfer function of a $1 \mathrm{~km}$ cable.

Second, different cables in length have different resonant frequencies shown in Figure 2-13. The first resonant frequency of a $50 \mathrm{~km}$ cable is around $350 \mathrm{~Hz}$ which is much lower than the one of a $1 \mathrm{~km}$ cable as shown in Figure 2-12.

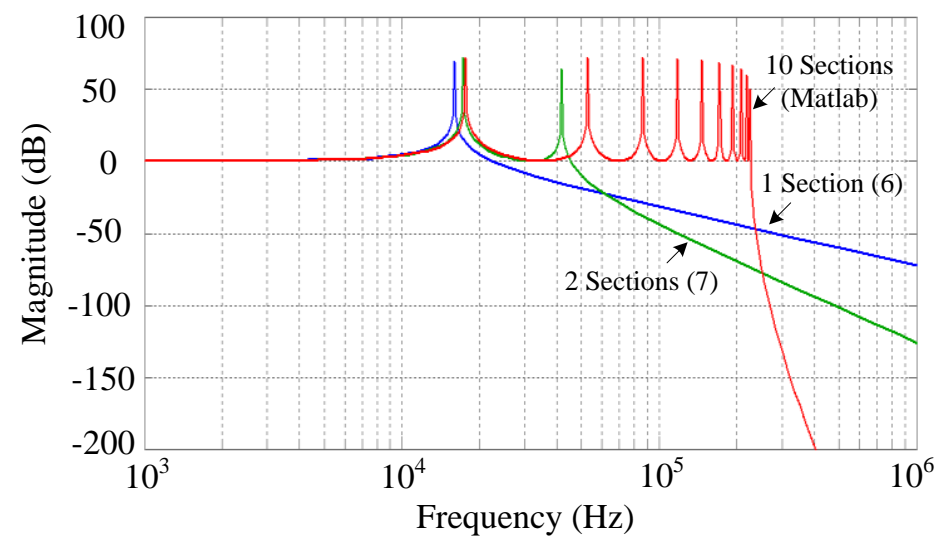

Figure 2-13 Magnitude response of the transfer function of $50 \mathrm{~km}$ and $48 \mathrm{~km}$ cables.

Therefore, from frequency domain perspective, the voltage at the end of the cable $\left(v_{d c_{-}} n^{\prime}\right)$ with respect to the one at the beginning of the cable $\left(v_{d c_{-}}\right)$can be defined as

$$
v_{d c_{-} n}{ }^{\prime}(t)=|H(j 2 \pi f)| v_{d c_{-} n}(t) \angle H(j 2 \pi f)=H_{n_{-} m} v_{d c_{-} n}\left(t+\Delta t_{n}+\Delta t_{c_{-} n}\right)
$$

where $|H(j \omega)|$ is the gain of magnitude, and $\angle H(j \omega)$ is the delay in phase. For simplicity, $H_{\mathrm{n} \_m}$ is used to represent the gain of magnitude of the m-order component for $v_{d c_{-} \mathrm{n},} \Delta t_{\mathrm{n}}$ is defined as 
same as before, and $\Delta t_{c_{\_} \mathrm{n}}$ is to represent the delay in phase. Note that $\Delta t_{c_{-} \mathrm{n}}$ is fixed given a fixed length of cable, that is

$$
\Delta t_{c_{-} n}=\frac{l_{c_{-} n}}{v}
$$

where $l_{c_{-}}$is the length of the cable between two adjacent offshore converters.

Applying (2-12) to (2-6) gives

$$
v_{d c_{-} n}{ }^{\prime}(t)=d_{n}+H_{n_{-} m} a_{n_{-} m} \sum_{m=1}^{\infty} \cos \left\{2 \pi m f\left(t+\Delta t_{n}+\Delta t_{c_{-} n}\right)\right\}
$$

\subsubsection{DC-link voltage}

As mentioned earlier, two items are mainly investigated in this part. One is dc-link current which affects the performance of the control objectives of the system and the other is dc-link voltage which determines the performance of the system in terms of insulation level and related cost. Two equivalent circuits can be derived depending on where to place the dc-link inductor.

(a) DC-link inductor evenly distributed at offshore converter side

One possible design is evenly placing the dc-link inductor at each offshore converter side as shown in Figure 2-14 where $L_{d c_{-} \mathrm{n}}=L_{d c} / \mathrm{n}$.

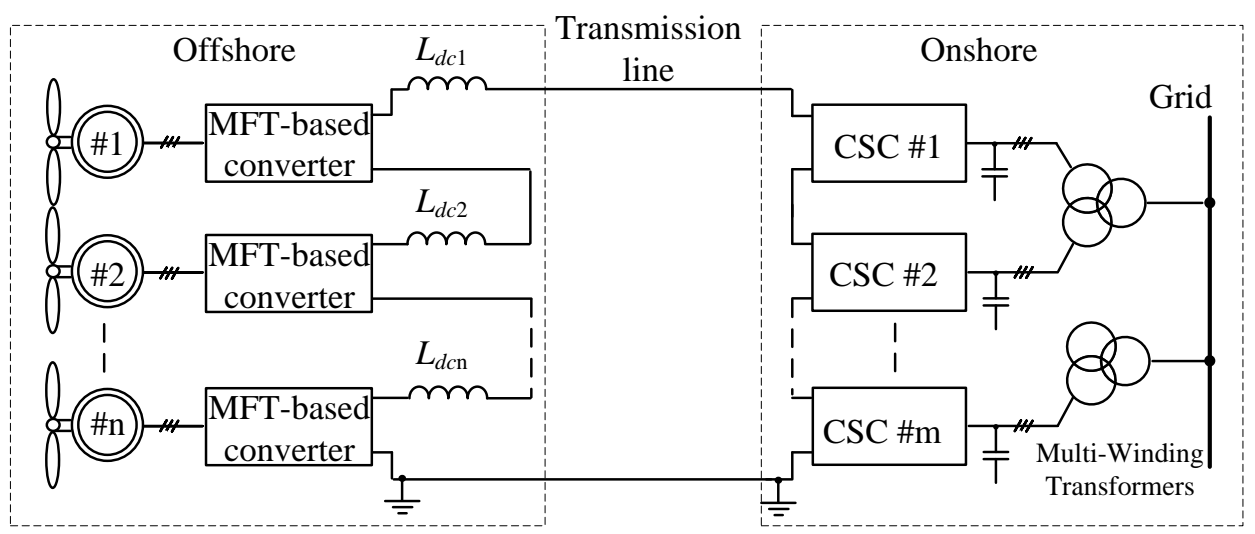

Figure 2-14 Configuration when evenly placing dc-link inductor at offshore side.

However, such a design is not allowed from the circuit perspective. As shown in its equivalent circuit Figure 2-15, the pulse-wave voltage source connected with inductor and the transmission line is equivalent to a voltage source seeing from the onshore CSCs. This voltage source is then 
connected in parallel with the capacitor at the output of the CSC which is not allowed, and the dc-link current control of the system under this condition is failed. This case is no longer discussed in the following.

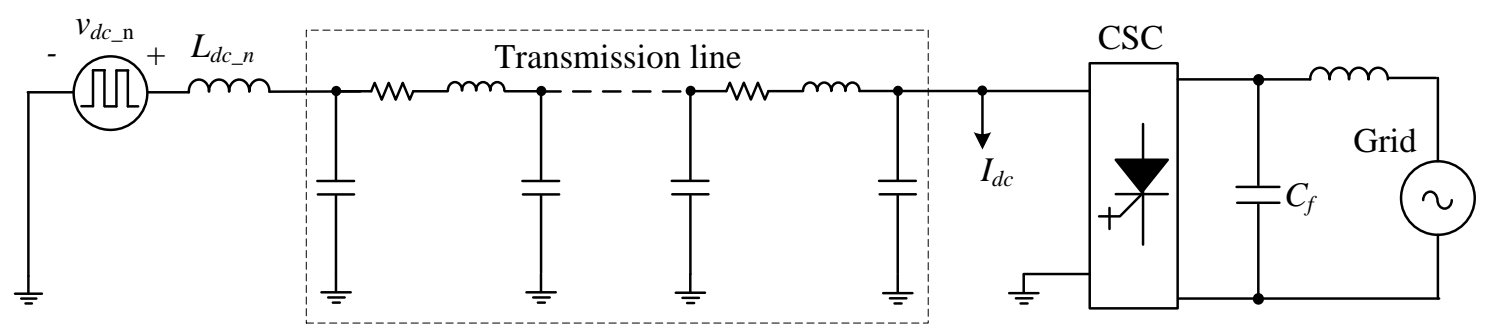

Figure 2-15 Equivalent circuit of Figure 2-14.

(b) DC-link inductor placed at onshore station

The system configuration when placing the dc-link inductor at onshore station is shown in Figure 2-1. Placing the dc-link inductor at the onshore station can greatly lower the burden of offshore construction. The equivalent circuit when placing the dc-link inductor at onshore station is shown in Figure 2-16. The cables between adjacent offshore converters $\left(l_{c_{-}} \mathrm{n}\right)$ and between offshore and onshore converters $\left(l_{C_{-} T}\right)$ are represented with RLC sections as mentioned previously. The onshore CSCs are equivalent to a pure resistor $R_{\text {load }}$ under the condition of unity power factor. The offshore converter \#n is replaced with a pulse-wave voltage source, that is $v_{d c \_n}$ as mentioned previously.

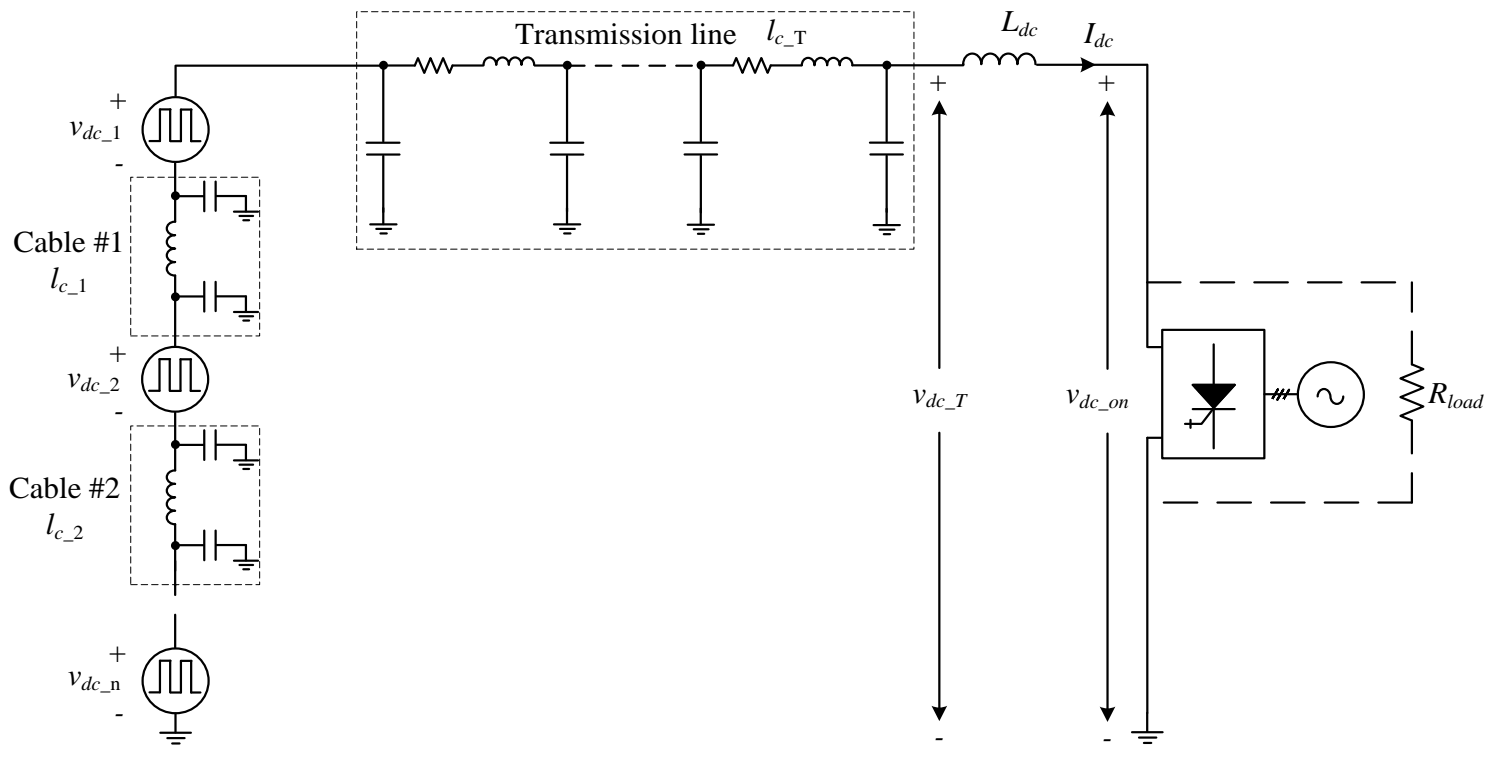

Figure 2-16 Equivalent circuit of the system when placing dc-link inductor at onshore station. 
The equivalent circuit shown in Figure 2-16 can be further simplified as shown in Figure 2-17 with the help of superposition and under the following assumptions. First, the interruptions between cables are neglected. Besides, the impedance of the DC-link inductor $\left(L_{d c}\right)$ together with the equivalent load $\left(R_{\text {load }}\right)$ are significantly larger than that of the cable, thus can be considered open circuited.

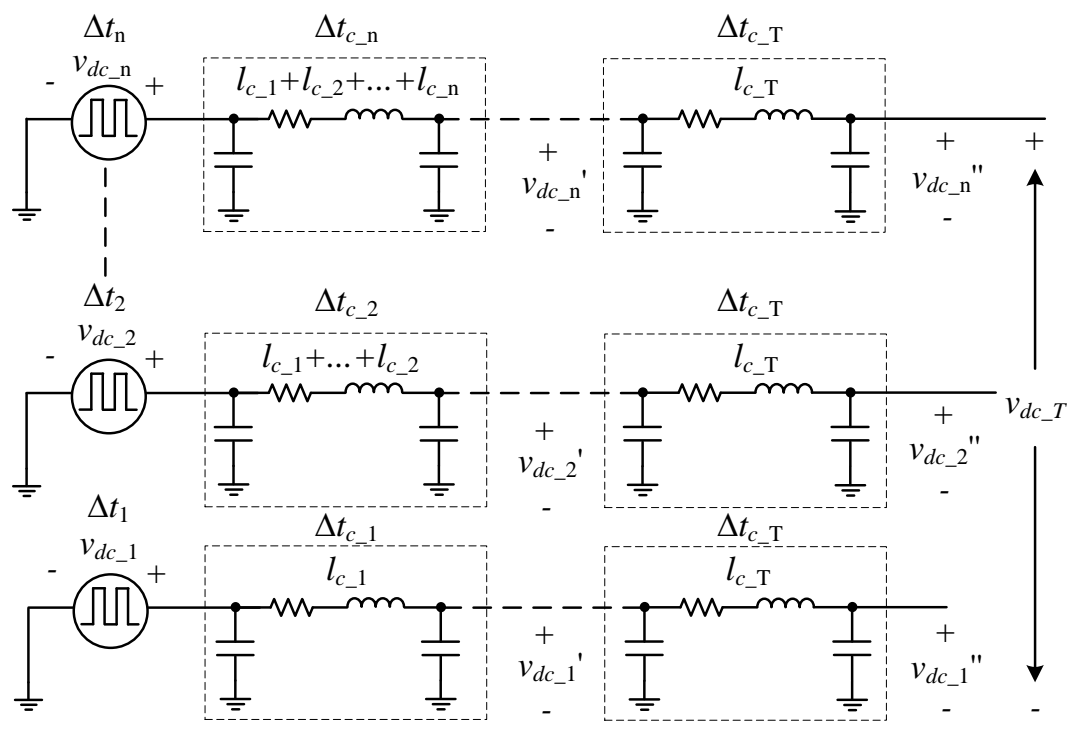

Figure 2-17 Simplification of Figure 2-16.

The voltage at the end of the transmission line $v_{d c_{-}}$, is expressed as

$$
v_{d c_{-} T}(t)=\sum_{n=1}^{n} v_{d c_{-} n} "
$$

where $v_{d c_{-} n} n$ is the individual voltage at the end of the transmission line transmitted from offshore to onshore. Shown in Fig. 11, the initial phase angle of $v_{d c_{-}} \mathrm{n}$ " consists of three parts: the initial phase angle of $v_{d c_{-} \mathrm{n}}\left(\Delta t_{\mathrm{n}}\right)$, the phase shift on the cable between offshore converters $\left(\Delta t_{c_{-} \mathrm{n}}\right)$, and the one on the cable between offshore and onshore converters $\left(\Delta t_{c_{-} \mathrm{T}}\right)$.

Substituting (2-14) into (2-15) yields 


$$
\begin{aligned}
& v_{d c_{-} T}(t)=\underbrace{d_{1}+H_{1_{-} m} a_{1_{-} m} \sum_{m=1}^{\infty} \cos \left\{2 \pi m f\left(t+\Delta t_{1}+\Delta t_{c_{-} 1}++\Delta t_{c_{-} T}\right)\right.}_{v_{d c_{-}} "} \\
& +\underbrace{d_{2}+H_{2_{-} m} a_{2_{-} m} \sum_{m=1}^{\infty} \cos \left\{2 \pi m f\left(t+\Delta t_{2}+\Delta t_{c_{-} 2}++\Delta t_{c_{-} T}\right)\right\}}_{v_{d c_{-} "} "}+ \\
& \ldots+\underbrace{d_{m}+H_{n_{-} m} a_{n_{-} m} \sum_{m=1}^{\infty} \cos \left\{2 \pi m f\left(t+\Delta t_{n}+\Delta t_{c_{-} n}++\Delta t_{c_{-} T}\right)\right\}}_{v_{d c_{-} n} "}
\end{aligned}
$$

It is difficult if not impossible to calculate the value of (2-16) in the full operation range as there is quite a number of uncertain variables. However, only the maximum peak value under rated condition needs to be considered in practice as it affects the insulation level, cost, and reliability of the system. Thus, it is very necessary to find the possible maximum peak value of (2-16) under rated condition.

Under rated condition, all the wind speeds at turbines are same and maintaining at $1 \mathrm{pu}$, which means the magnitudes and duty cycles of all the pulse-wave voltages $v_{d c_{-} \mathrm{n}}$ are same at $1 \mathrm{pu}$. Besides, the time delay (phase shift) on the cable $\Delta t_{c_{-} \mathrm{n}}$ is known once the cable is fixed, and so does for $\Delta t_{c_{-} T}$. The gain of magnitude for the m-order ac component $H_{\mathrm{n}_{-} \mathrm{m}}$ which depends the characteristics of the cable can also be estimated. The initial phase angles $\left(\Delta t_{\mathrm{n}}\right)$, on the other hand, cannot be guaranteed to be identical, which is already analyzed earlier, thus not repeated here. To sum up, all the variables in (2-16) can be known or can be estimated except the initial phase angle $\Delta t_{\mathrm{n}}$ of $v_{d c_{-} \mathrm{n}}$.

As mentioned earlier, $\Delta t_{\mathrm{n}}$ is varying from 0 to $1 / 2400$. Then, a lot of possibilities could happen on (2-16) where the maximum value occurs at the condition of

$$
\left(t+\Delta t_{1}+\Delta t_{c_{-} 1}+\Delta t_{c_{-} T}\right)=\left(t+\Delta t_{2}+\Delta t_{c_{-} 2}++\Delta t_{c_{-} T}\right)=\cdots=\left(t+\Delta t_{n}+\Delta t_{c_{-} n}+\Delta t_{c_{-} T}\right)
$$

when all the individual voltages at the end of the transmission line $v_{d c_{-}} n$ are in phase.

Removing the common parts in (2-17) gives

$$
\left(\Delta t_{1}+\Delta t_{c_{-} 1}\right)=\left(\Delta t_{2}+\Delta t_{c_{-} 2}\right)=\cdots=\left(\Delta t_{n}+\Delta t_{c_{-} n}\right)
$$

which means when the difference in initial phase angles between $v_{d c_{-} \mathrm{n}}$ and $v_{d c_{-}}$, that is $\Delta t_{\mathrm{n}}-\Delta t_{1}$, is equal to the difference on the cables, that is $\Delta t_{c_{-} n}-\Delta t_{c_{-} 1},(2-16)$ receives its maximum value. On 
this basis, the equivalent circuit can be simplified further as shown in Figure 2-18 where $v_{d c_{-} n}$ ' is defined as same as that in Figure 2-17.

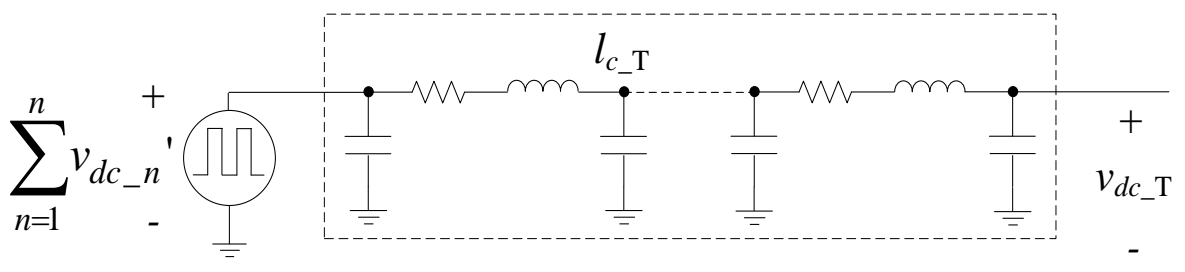

Figure 2-18 Simplification of Figure 2-17.

In addition, shown in Figure 2-17, the maximum peak value for individual voltages at the end of the cable $v_{d c_{-} n}$ " could be two times of that of $v_{d c_{-} \mathrm{n}}$ as the voltage reflection coefficient is $\Gamma_{l}=1$ under an open circuit. Therefore, the resultant maximum peak value of the dc-link voltage is two times of the rated value. The average value, however, is maintained.

The dc-link current, on the other hand, is not affected. As mentioned earlier, the possible maximum peak value of the dc-link voltage that could be two times of the rated value is determined by those ac components where the fundamental frequency is $2400 \mathrm{~Hz}$. Such ac components produce significantly high inductive reactance on the dc-link inductor. Thus, the resultant ac current in the dc-link current is too small that can be neglected. To sum up, the possible maximum peak value of the dc-link voltage when placing the dc-link inductor at onshore station could be two times of the rated value under the condition of (2-18). The dc-link current is not affected.

Figure 2-19 illustrates the simulated performance of the dc-link voltage and current under the condition of (2-18). Five offshore turbine units are employed. The initial phase angles for $v_{d c_{-} 1}$, $v_{d c_{-} 2} \ldots$, and $v_{d c_{-} 5}$ are purposely set to $0, \Delta t_{2}, \Delta t_{3} \ldots$, and $\Delta t_{5}$ to meet the condition of (2-18). For example, $\Delta t_{2}$ is the time delay on the cable connected between $v_{d c_{-} 1}$ and $v_{d c_{-} 2}$, while $\Delta t_{5}$ is the time delay on the cable between $v_{d c_{-} 1}$ and $v_{d c_{-} 5}$. As shown in Figure 2-19, the peak value of the dc-link voltage is two times of its rated value. The dc-link current, on the other hand, is not affected. Figure 2-20 illustrates the simulated performance of the dc-link voltage and current without the condition of (2-18). The initial phase angles for $v_{d c_{-} 1}, v_{d c_{-} 2} \ldots$, and $v_{d c_{-} 5}$ are purposely set to be same, 0, for example. As shown in Figure 2-20, the peak value of the dc-link voltage is around 1.6 times of its rated value which is lower than that in Figure 2-19. 

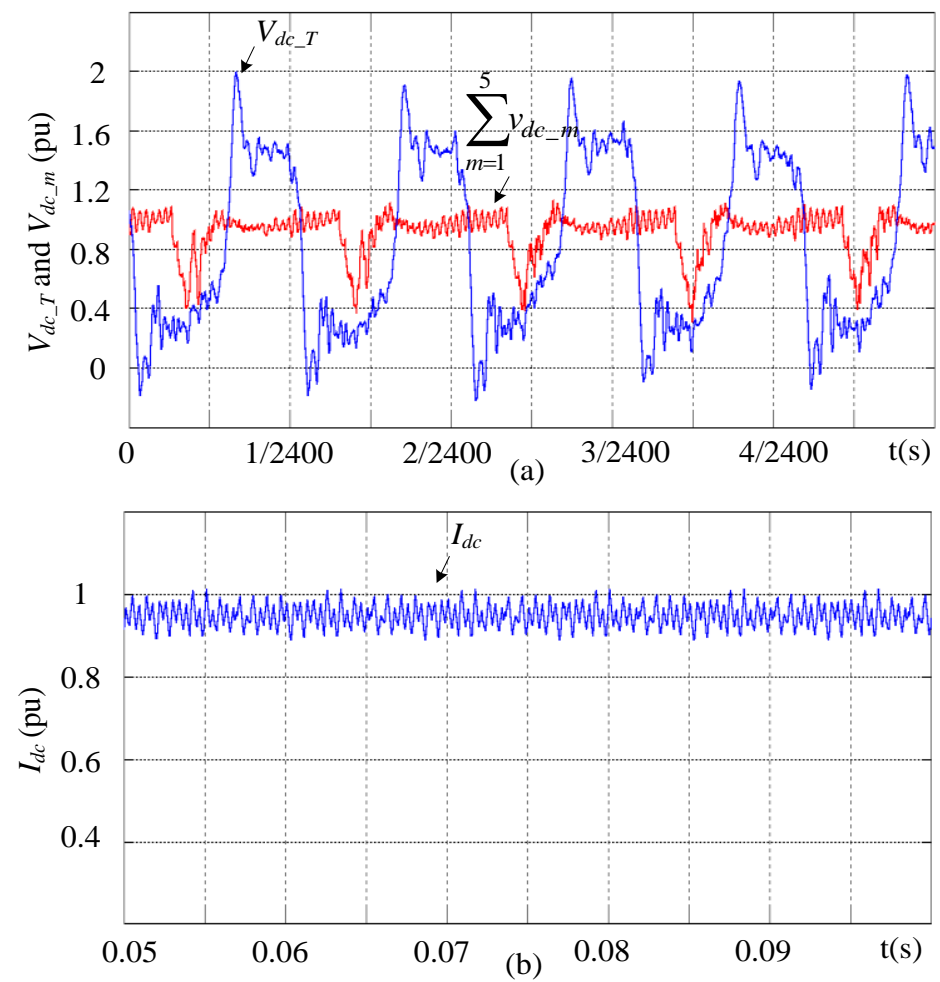

Figure 2-19 DC-link voltage and dc-link current under the condition of (2-18).
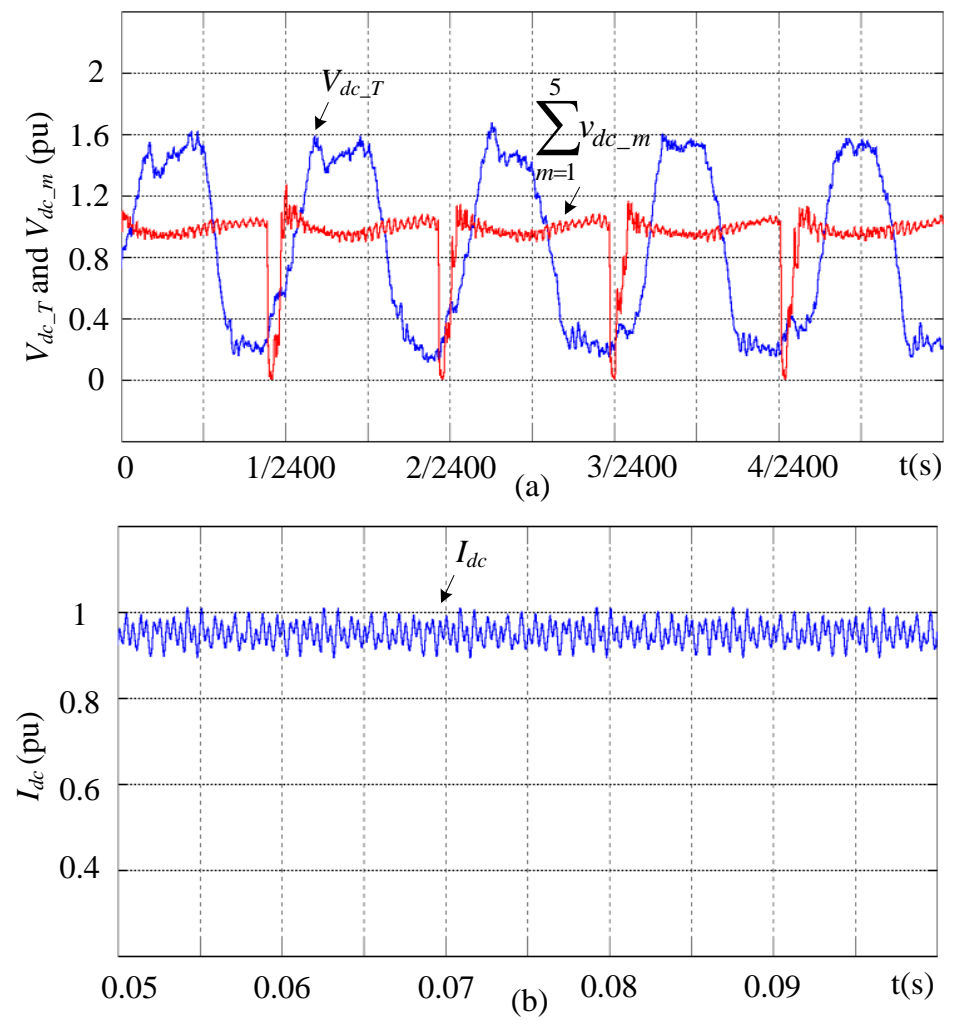

Figure 2-20 DC-link voltage and dc-link current without the condition of (2-18). 


\subsubsection{DC filter design}

To avoid the possible two times of rated value of the dc-link voltage, a dc filter is added to each offshore converter as shown in Figure 2-21. On this basis, the design of dc filter and dc-link inductor are given.

The dc filter helps remove the ac components from the pulse-wave voltage $v_{d c_{-}}$and keeps its dc component. All the dc components are then connected in series to reach an HVDC level and then transmitted to the onshore converters through the cables. However, it is worth noting that the operation principle of the configuration with de filter shown in Figure 2-21 is different from VSC-based configurations. In VSC-based configurations, the dc-link voltage should be maintained as a constant in the full operation range and thus should be evenly distributed among the dc-link capacitors. Here, in the CSC-based configuration, it is not the case at all. First, the capacitors are used for filter purpose. Second, the voltage across the capacitors, $V_{d c_{-} T \mathrm{n}}$, is decoupled with each other, and its value is depending on the wind speed at the turbine. There is no voltage balancing issue among the series-connected dc-filters capacitors.

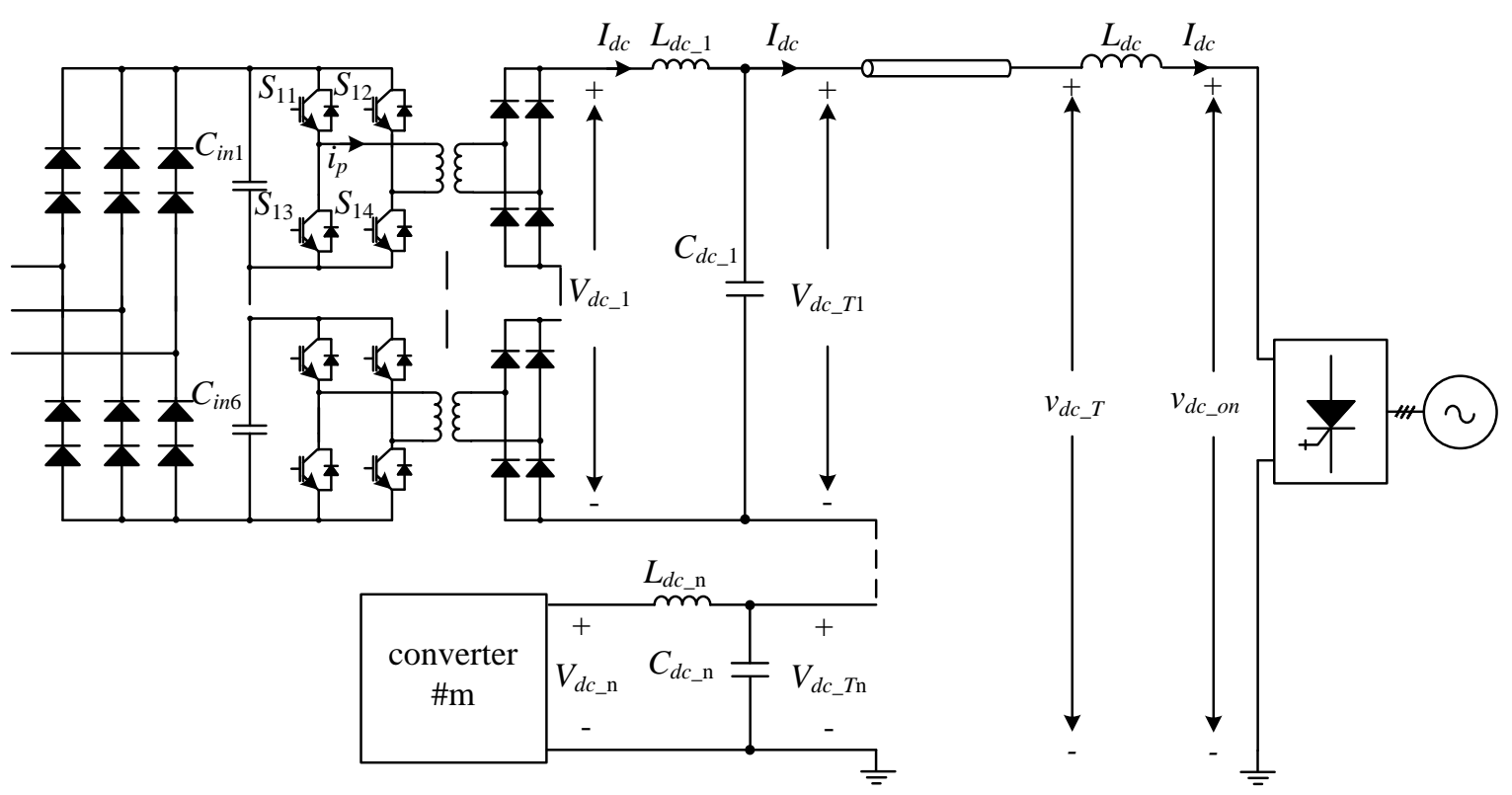

Figure 2-21 Configuration with dc filter.

The dc filter is designed by the voltage-second balance principle shown in Figure 2-22, that is

$$
\left(V_{d c_{-} n}-V_{d c_{-} T n}\right)=L_{d c_{-} n} \frac{\Delta I_{d c}}{\Delta t}
$$

where $V_{d c_{-} T \mathrm{n}}=V_{d c_{-} T} / \mathrm{n}$ under rated condition, $V_{d c_{-} T}$ is the average dc-link voltage, $\Delta I_{d c}$ is the rated 
dc-link current ripple, $\Delta t$ is the duty cycle under rated condition, and $V_{d c_{-} \mathrm{n}}$ is defined as same as before.

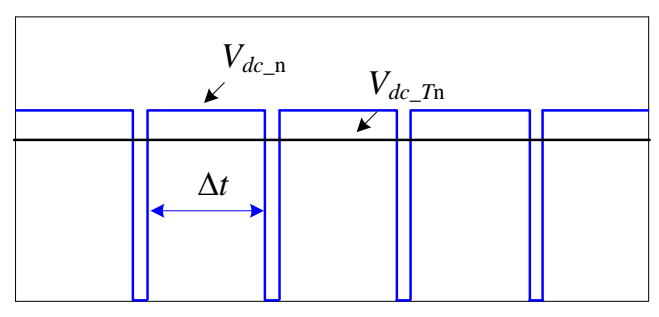

Figure 2-22 Voltage across each offshore converter.

Equation (2-19) gives the value of the filter inductor $L_{d c_{-} \mathrm{n}}$, the capacitor $C_{d c_{-} \mathrm{n}}$ is then depending on the selection of the cutoff frequency of the dc filter, that is

$$
f_{\text {filter }}=\frac{1}{2 \pi \sqrt{L_{d c_{-} n} C_{d c_{-} n}}}
$$

The cutoff frequency of the dc filter $f_{\text {filter }}$ should be selected based on the worst case, that is when one of the resonant frequencies of the cable model is just locating at the fundamental frequency $(2400 \mathrm{~Hz})$. For example, for a $50 \mathrm{~km}$ cable, the gain for the fundamental component $(2400 \mathrm{~Hz})$ is around $40 \mathrm{~dB}$ (100 times higher). Thus, the attenuation coefficient of the dc filter under $2400 \mathrm{~Hz}$ should be at least $40 \mathrm{~dB}$ (100 times lower) in order to cancel the above possible amplification on the cable. On this basis, the cutoff frequency $f_{\text {filter }}$ is selected to be $300 \mathrm{~Hz}$. Note that the detailed calculation of the filter parameters is not presented just for conciseness as it is a very well proven method.

Figure 2-23 shows the dc-link voltage and current with the dc filter. The main harmonics of the voltage $v_{d c_{-} n}$ are highly attenuated by the dc filter, while they are amplified by the transmission line. Thus, the dc-link voltage at the end of the transmission line $V_{d c_{-} \mathrm{T}}$ still contains some loworder harmonics. The peak value of the dc-link voltage with the dc filter is around 0.45 times of that without the dc filter, thus giving significant cost saving on insulation issues and cables and increasing system reliability. 

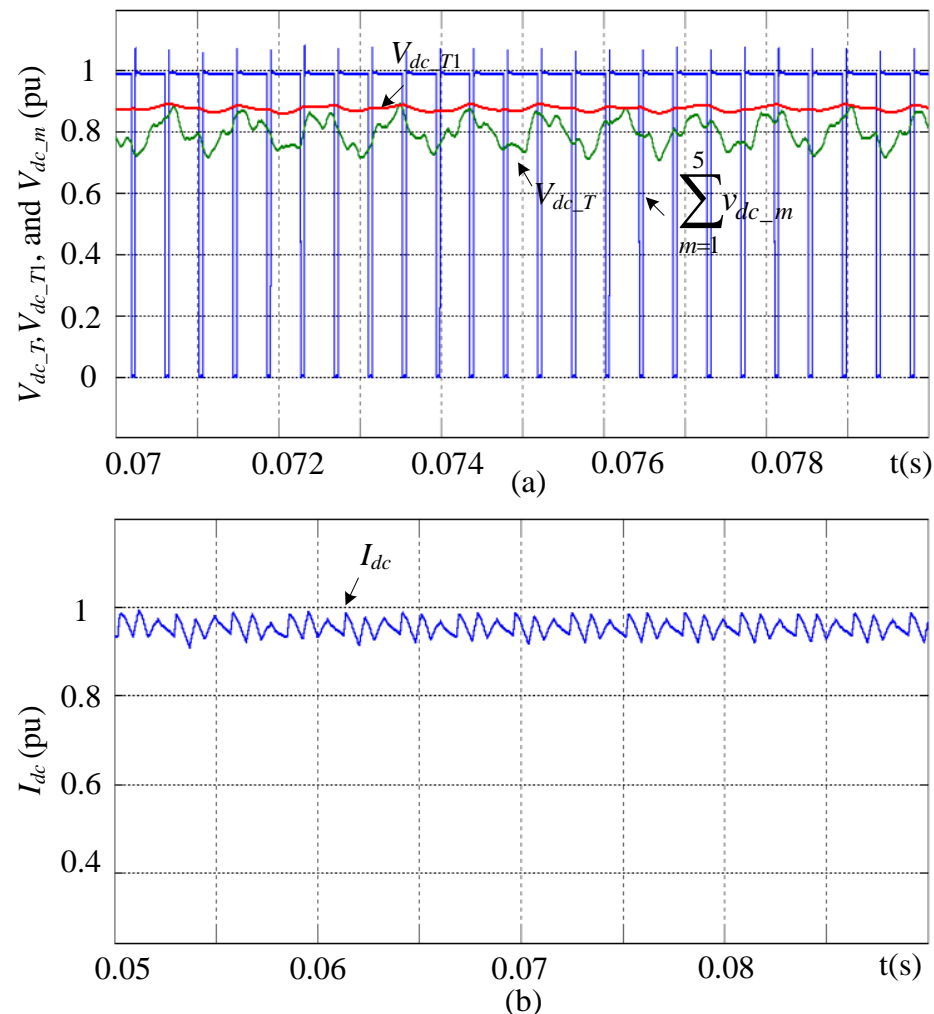

Figure 2-23 DC-link voltage and dc-link current with DC filter.

\subsection{Performance investigation}

The performance of the proposed configurations and the effectiveness of the proposed voltage/current balancing scheme are verified by MATLAB/Simulink simulations. The wind turbine and PMSG used in simulation are provided by MATLAB/Simulink. The turbine model receives the wind speed and provides an optimized reference speed to the control system. The inertia constant of a megawatts-level WECS is normally around a few seconds [38], while in the following simulations, it is reduced to achieve a faster speed.

\subsubsection{Voltage balance control for MV configuration}

Five modules are employed in the modular MFT-based converter. To introduce imbalance, the turn ratios of the five transformers are purposely set to 1:1 (Module 1), 1:1.005 (Module 2), and 1:1.01 (Module 3), 1:1.015 (Module 4), and 1:1.02 (Module 5). In the following simulation, three typical operation conditions are conducted: with/without voltage balance control, stepped change in wind speed, and reactive power control. 
(a) Performance with and without voltage balance control

Figure 2-24 illustrates the simulated performance of the proposed WECS under conditions of unity power factor (UPF) and with/without voltage balance control scheme. Figure 2-24 (a) shows grid-side injected real and reactive power $\left(P_{s}, Q_{s}\right)$; Figure 2-24 (b) dc-link current $I_{d c}$; and Figure 2-24 (c) input capacitor voltages of the MFT-based cascaded dc-dc converter. Before $t=2$ $\mathrm{s}$, the system operates in steady state with voltage balance control. The grid-side injected real and reactive power are $1 \mathrm{MW}$ and $0 \mathrm{MVar}$, respectively. The input voltage of each module is balanced with $V_{c 1}=V_{c 2}=V_{c 3}=V_{c 4}=V_{c 5}=1000 \mathrm{~V}$. At $t=2 \mathrm{~s}$, the voltage balance control is deactivated (all five modules operate under the common duty cycle $d_{\text {com}}$ ). The dc-link current and the real/reactive power are still controlled well, while the input capacitor voltages begin to diverge. $V_{c 1}$ is the highest $(1180 \mathrm{~V})$ among the voltages because the duty ratio of its transformer (Module 1) is the lowest of the obtained ratios. At $t=2.5 \mathrm{~s}$, the voltage balance control scheme is reactivated and the input capacitor voltages quickly converge to nominal values.
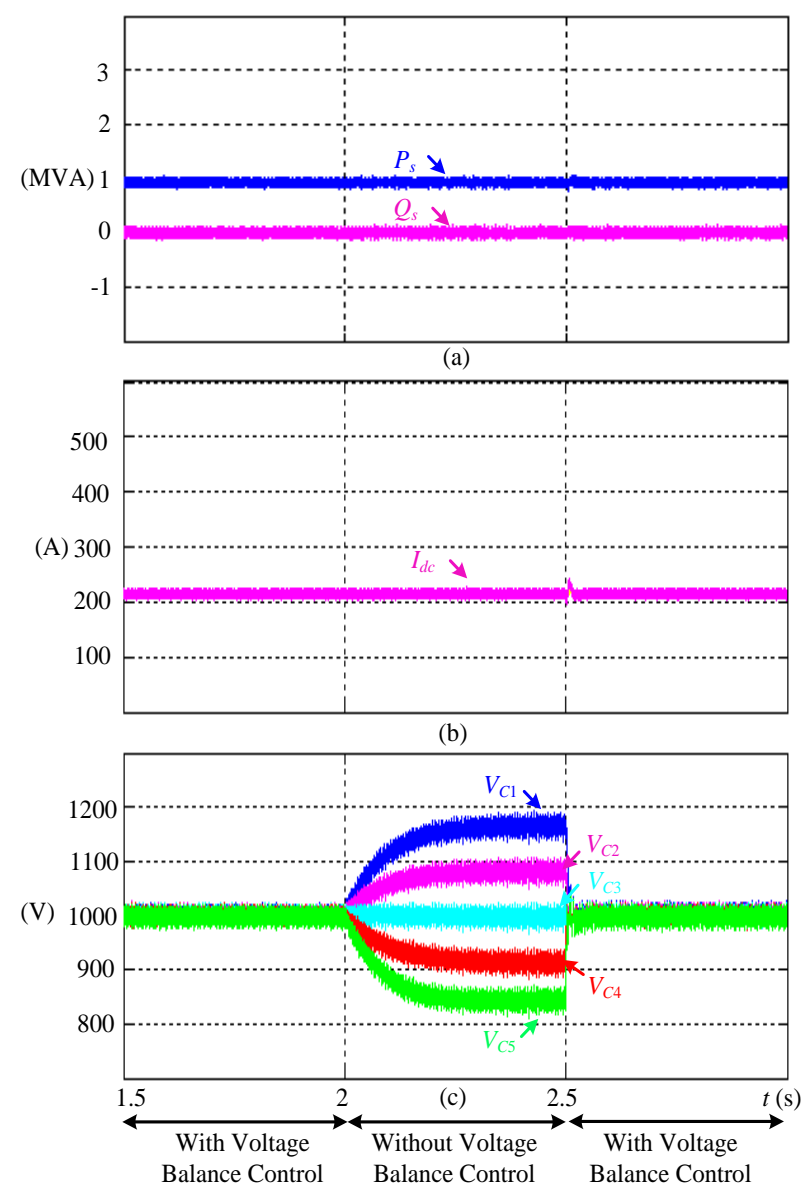

Figure 2-24 Simulated performance with and without voltage balance control. 


\section{(b) Performance under stepped wind speed}

Figure 2-25 illustrates the simulated performance of the proposed WECS under stepped wind speed. To emulate the transient response of the proposed WECS, wind speed is purposely stepped down from $12 \mathrm{~m} / \mathrm{s}(1 \mathrm{pu})$ to $10 \mathrm{~m} / \mathrm{s}(0.833 \mathrm{pu})$ at $t=2 \mathrm{~s}$ and up from $10 \mathrm{~m} / \mathrm{s}(0.833 \mathrm{pu})$ to $12 \mathrm{~m} / \mathrm{s}(1 \mathrm{pu})$ at $t=4 \mathrm{~s}$, respectively. The speed of the PMSG, that is $w_{g}$, tracks the reference speed $w_{g_{-}} r e f$ well in both steady and transient states. As the power, $P_{s}$ is the cube of generator speed $w_{g}$, only $0.57 \mathrm{pu}$ of power is captured when the wind speed is reduced to $0.833 \mathrm{pu}$ as shown in Figure 2-25 (a). To minimize loss, a minimum dc-link current is regulated accordingly shown in Figure 2-25 (b). The input capacitor voltages $\left(V_{c 1}, V_{c 2}, V_{c 3}, V_{c 4}\right.$, and $\left.V_{c 5}\right)$ are well balanced in all steady and transient states, as shown in Figure 2-25 (c).

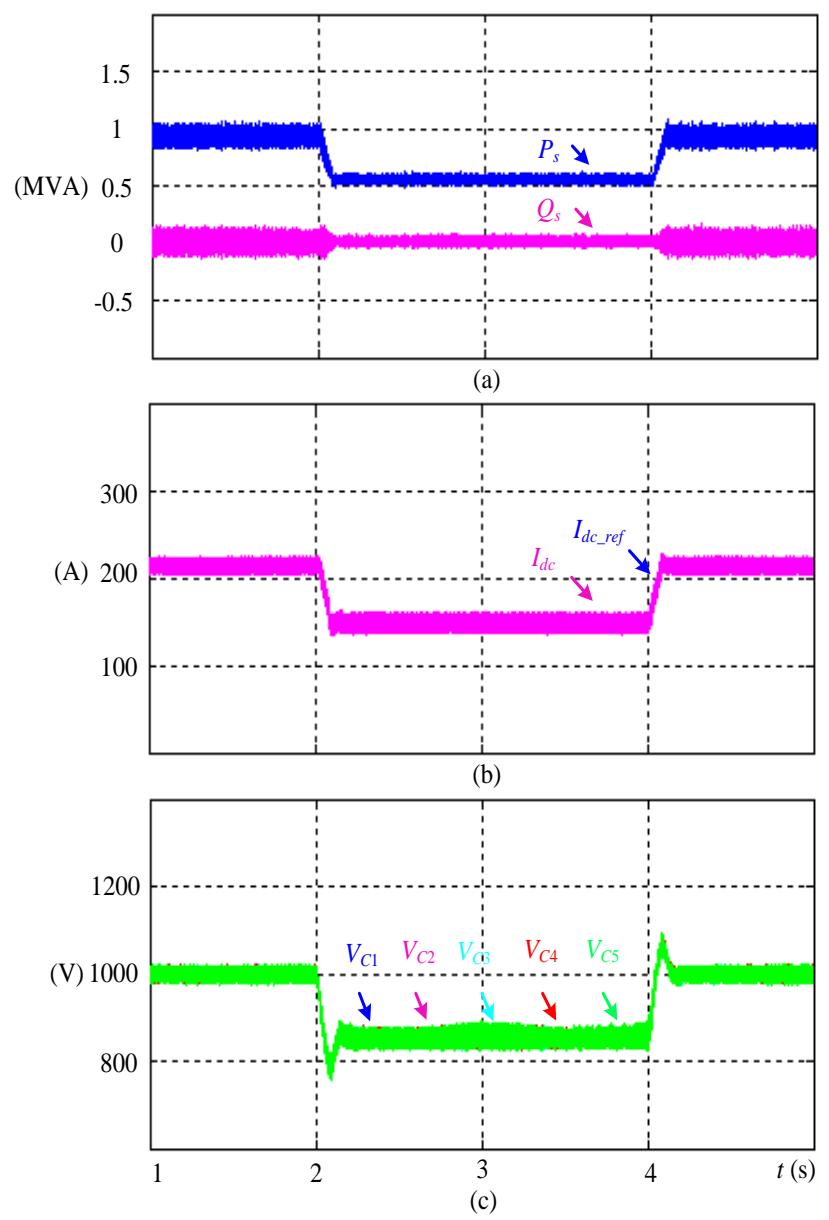

Figure 2-25 Simulated performance under stepped wind speed. 
(c) Performance under reactive power control

Figure 2-26 presents the simulated performance of the proposed WECS under reactive power control. Reactive power $Q_{s}$ is injected into the grid according to grid codes [3] with $330 \mathrm{kVar}$ $(0.33 \mathrm{pu})$ between 2 and $3 \mathrm{~s}$, and $-330 \mathrm{kVar}(-0.33 \mathrm{pu})$ between 4 and $5 \mathrm{~s}$, respectively. As shown in Figure 2-26 (b), a minimum dc-link current is set accordingly to minimize loss in the process. Figure 2-26 (c) shows that all input capacitor voltages are evenly distributed. The power factors under reactive power injection $(0 \mathrm{pu}, 0.33 \mathrm{pu},-0.33 \mathrm{pu})$ are $1,0.95$, and -0.95 as shown in Figure 2-27. $v_{a}$ and $i_{g a}$ are the grid-side phase voltage and the injected current, respectively.

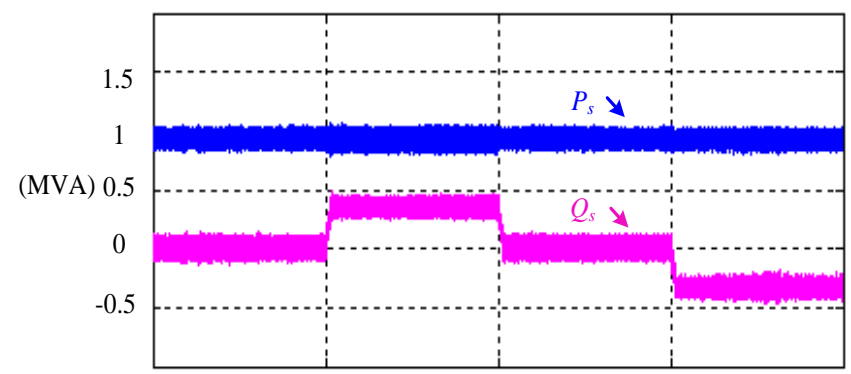

(a)

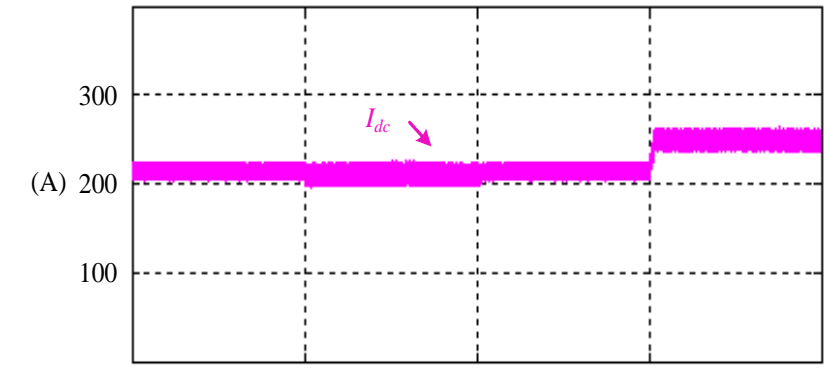

(b)

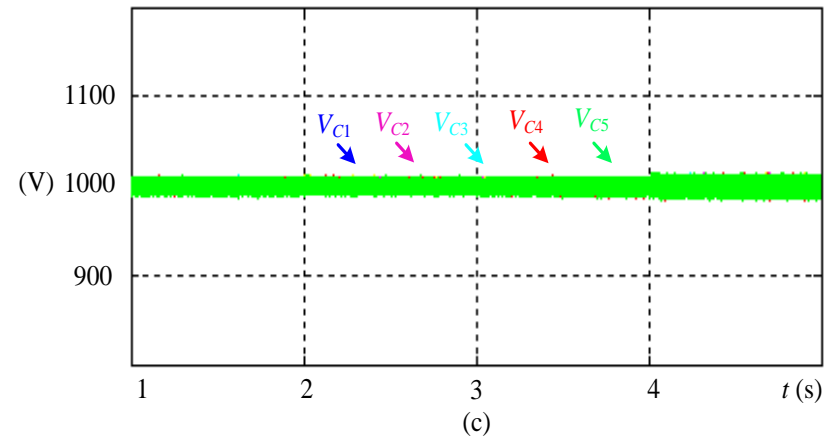

Figure 2-26 Simulated performance under reactive power control. 


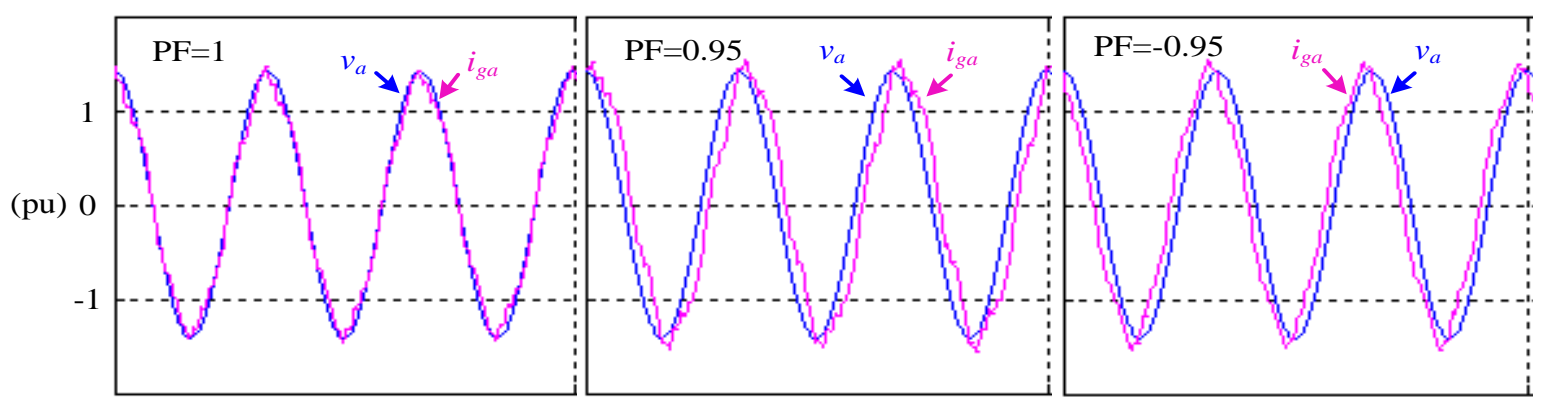

Figure 2-27 Grid injected current and phase voltage.

\subsubsection{Current balance control for $\mathrm{LV}$ configuration}

Six modules are employed in the offshore modular converter to cooperate with the use of a $4160 \mathrm{~V}$ CSC. To introduce imbalance, the turn ratios of the six transformers used are purposely set to 1:1 (Module 1, 2, and 3), 1:1.2 (Module 4, 5, and 6). Note that the average input currents $\bar{I}_{m N}$ of the constituent modules are used and presented in the following.

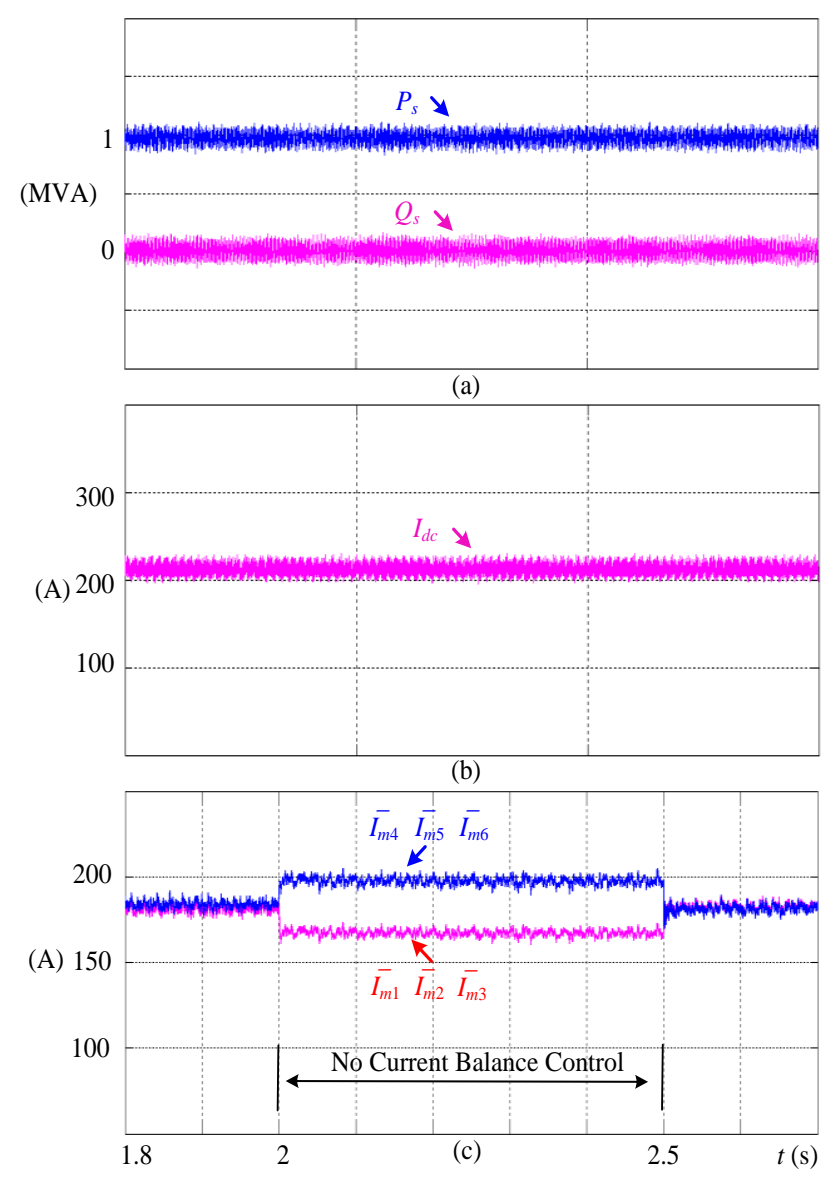

Figure 2-28 Simulated performance with/without current balance control. 
(a) Performance with and without voltage balance control

Figure 2-28 illustrates the simulated performance of the proposed topology with/without average current balance control. Figure 2-28 (a) shows the grid-side injected real and reactive power $\left(P_{s}, Q_{s}\right)$; Figure 2-28 (b) dc-link current $\left(I_{d c}\right)$; and Figure 2-28 (c) average input currents with/without current sharing control $\left(I_{m}\right)$. The system is operating under rated condition where the grid-side injected real and reactive power $\left(P_{s}, Q_{s}\right)$ are $1 \mathrm{MW}$ and $0 \mathrm{MVar}$. Before time $t=2 \mathrm{~s}$, the current balance control is employed. The average input currents are balanced as shown in Figure 2-28 (c). At time $t=2 \mathrm{~s}$. the current control scheme is deactivated that all the modules operate under the common duty cycle. Then, the average input currents begin to diverge and finally reach to a steady state. At $t=2.5 \mathrm{~s}$, the current balance control is reactivated and the input currents of all the modules quickly converge to normal values again.

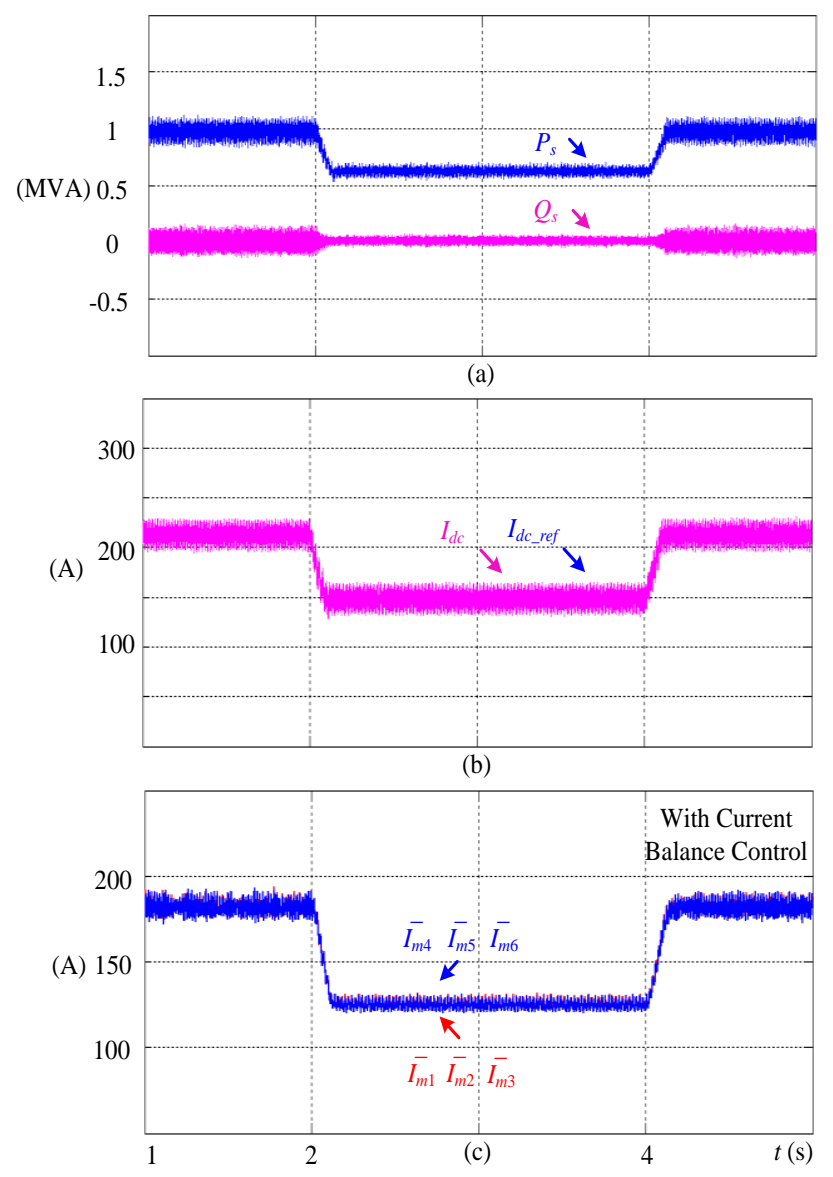

Figure 2-29 Simulated performance under stepped wind speed.

(b) Performance under stepped wind speed 
Figure 2-29 illustrates the simulated performance under stepped wind speed. To simulate the transient response, the wind speed is purposely set to step down and up from $12 \mathrm{~m} / \mathrm{s}$ (1 pu) to 10 $\mathrm{m} / \mathrm{s}(0.833 \mathrm{pu})$ at $t=2 \mathrm{~s}$ and from $10 \mathrm{~m} / \mathrm{s}(0.833 \mathrm{pu})$ back to $12 \mathrm{~m} / \mathrm{s}(1 \mathrm{pu})$ at $t=4 \mathrm{~s}$, respectively. The reactive power $Q_{s}$, on the other hand, is maintained at $0 \mathrm{pu}$. As mentioned earlier, a minimum dc-link current is altered accordingly to minimize the loss. During the transient and steady states, the input average currents of the modular converter are balanced as shown in Figure 2-29 (c).

(c) Performance under reactive power control

Figure 2-30 presents the simulated performance under stepped reactive power injection. Reactive power $Q_{s}$, is injected into the grid according to grid codes [3] with $330 \mathrm{kVar}(0.33 \mathrm{pu})$ between 2 and $3 \mathrm{~s},-330 \mathrm{kVar}(-0.33 \mathrm{pu})$ between 4 and $5 \mathrm{~s}$, respectively. The input average currents of the modular converter are balanced well during the process.
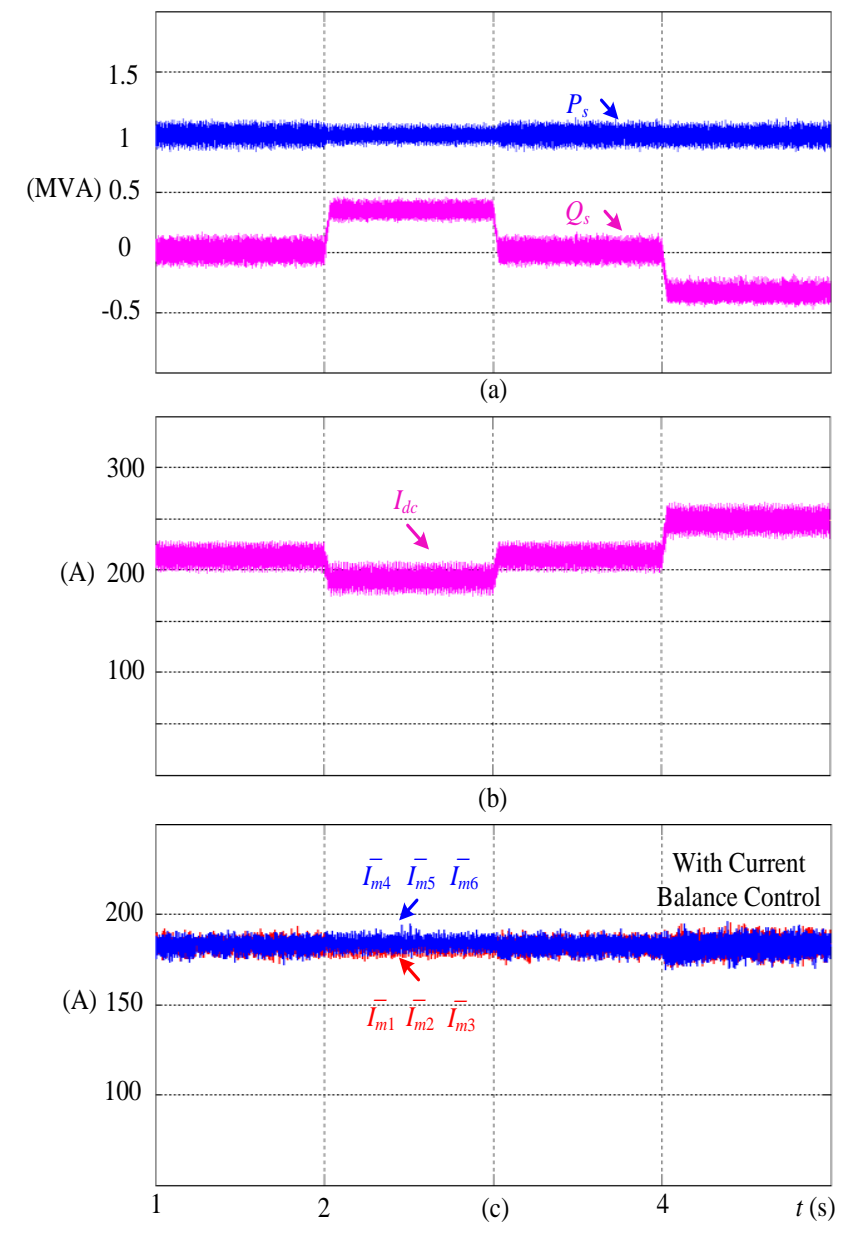

Figure 2-30 Simulated performance under stepped reactive power control. 


\subsection{Experimental verification}

Experiments are conducted to verify the performance of the proposed configuration and the control schemes. First, the experiment setup is simplified by replacing the wind turbine-PMSGdiode rectifier part with a voltage supply. All the control schemes are processed based on dSPACE DS1103. Detailed system parameters are listed in the Appendix.

\subsubsection{Experimental verification for MV configuration}

Three MFT modules are employed in the experiment, and the turn ratios of the three MFTs are set to $1: 1$ (with a tolerance of $\pm 2 \%$ ). A 400 -ohm resistor is paralleled with the input capacitor of Module 3 on purpose to introduce imbalance.

(a) Performance with and without voltage balance control

Figure 2-31 shows the experimental performance of the configuration with and without the voltage balance control scheme. $V_{i n}$ is the total input voltage; $V_{c 1}, V_{c 2}$, and $V_{c 3}$ are the input capacitor voltages for Module 1, 2, and 3, respectively; $I_{d c}$ is the measured dc-link current; and $P_{s}$ and $Q_{s}$ are the plotted real and reactive power based on the measured voltages and currents data. With voltage balance control, the input voltages of the modules are well balanced with $V_{c 1}=V_{c 2}$ $=V_{c 3}=83 \mathrm{~V}$; the dc-link current is controlled at $8.5 \mathrm{~A}$; and the grid-side injected real and reactive power are 1,275 W and 0 VA. As depicted in Figure 2-31 (a), when the voltage balance control is deactivated (all the three modules operate under common duty cycle $d_{\text {com }}$ ), the input capacitor voltages $\left(V_{c 1}, V_{c 2}\right.$, and $\left.V_{c 3}\right)$ start diverging. $V_{c 3}$ decreases to be the lowest value due to its paralleled resistor (400 ohm); $V_{c 1}$ and $V_{c 2}$ are unequal because of the tolerance in the turns ratio of the transformers $( \pm 2 \%)$. The dc-link current and the real/reactive power, on the other hand, are tracking their references well. The input capacitor voltages of the modular converter quickly converge to nominal values when the voltage balance control scheme is reactivated. 

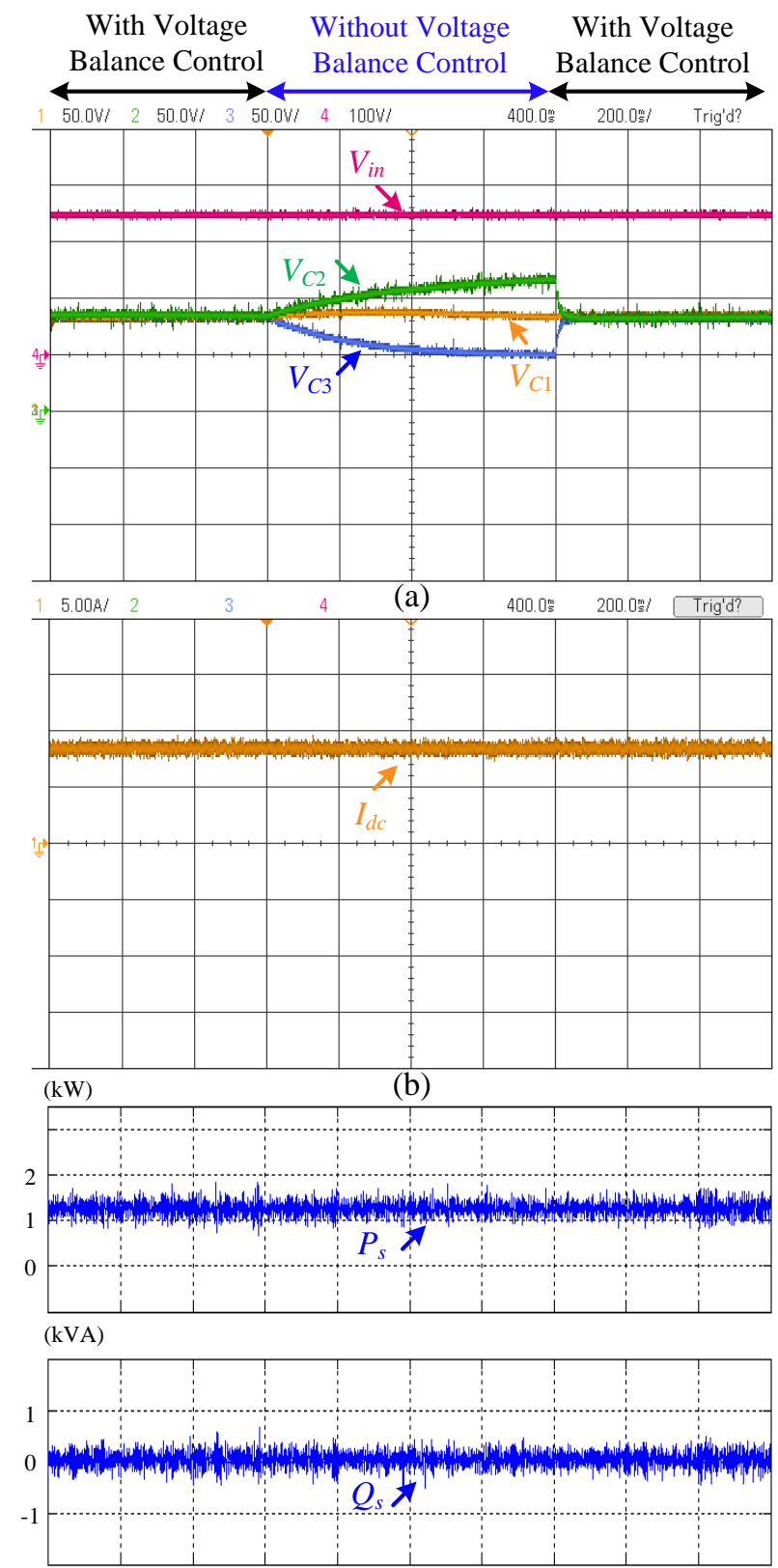

(c)

Figure 2-31 Experimental performance with/without voltage balance control. 

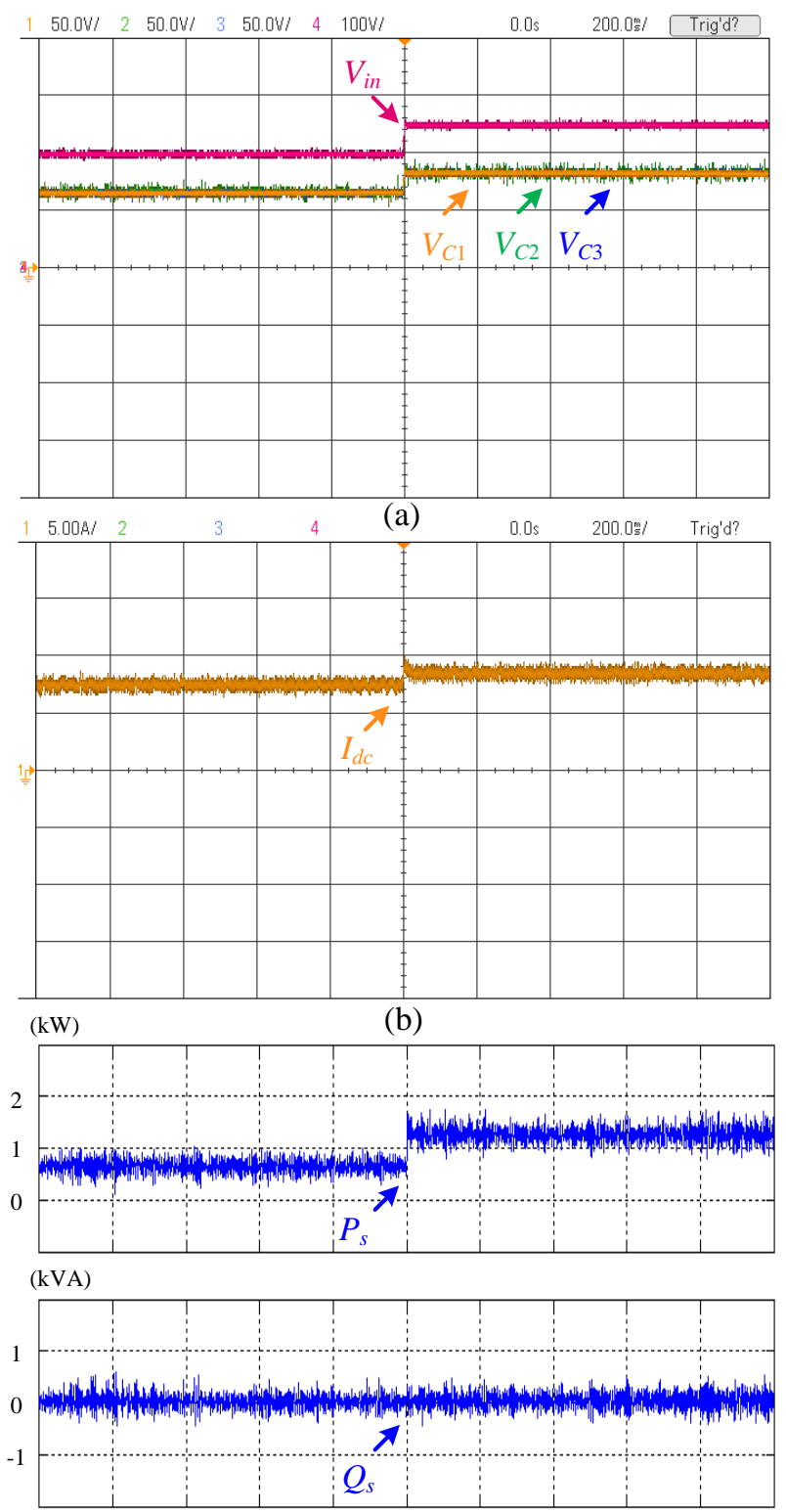

(c)

Figure 2-32 Simulated performance under stepped input voltage.

(b) Performance under stepped wind speed

Figure 2-32 illustrates the experimental performance under stepped input voltage from $0.8 \mathrm{pu}$ $(200 \mathrm{~V})$ to $1 \mathrm{pu}(250 \mathrm{~V})$. The resultant dc-link current $I_{d c}$ increases from $7.5 \mathrm{~A}$ to $8.5 \mathrm{~A}$, and the injected real power $P_{s}$ increases from approximately $0.52 \mathrm{pu}(660 \mathrm{~W})$ to $1 \mathrm{pu}(1275 \mathrm{~W})$ while the reactive power $Q_{s}$ is maintained at zero. During both steady and transient states, the input capacitor voltages $\left(V_{c 1}, V_{c 2}\right.$, and $\left.V_{c 3}\right)$ are well balanced as shown in Figure 2-32 (a). 

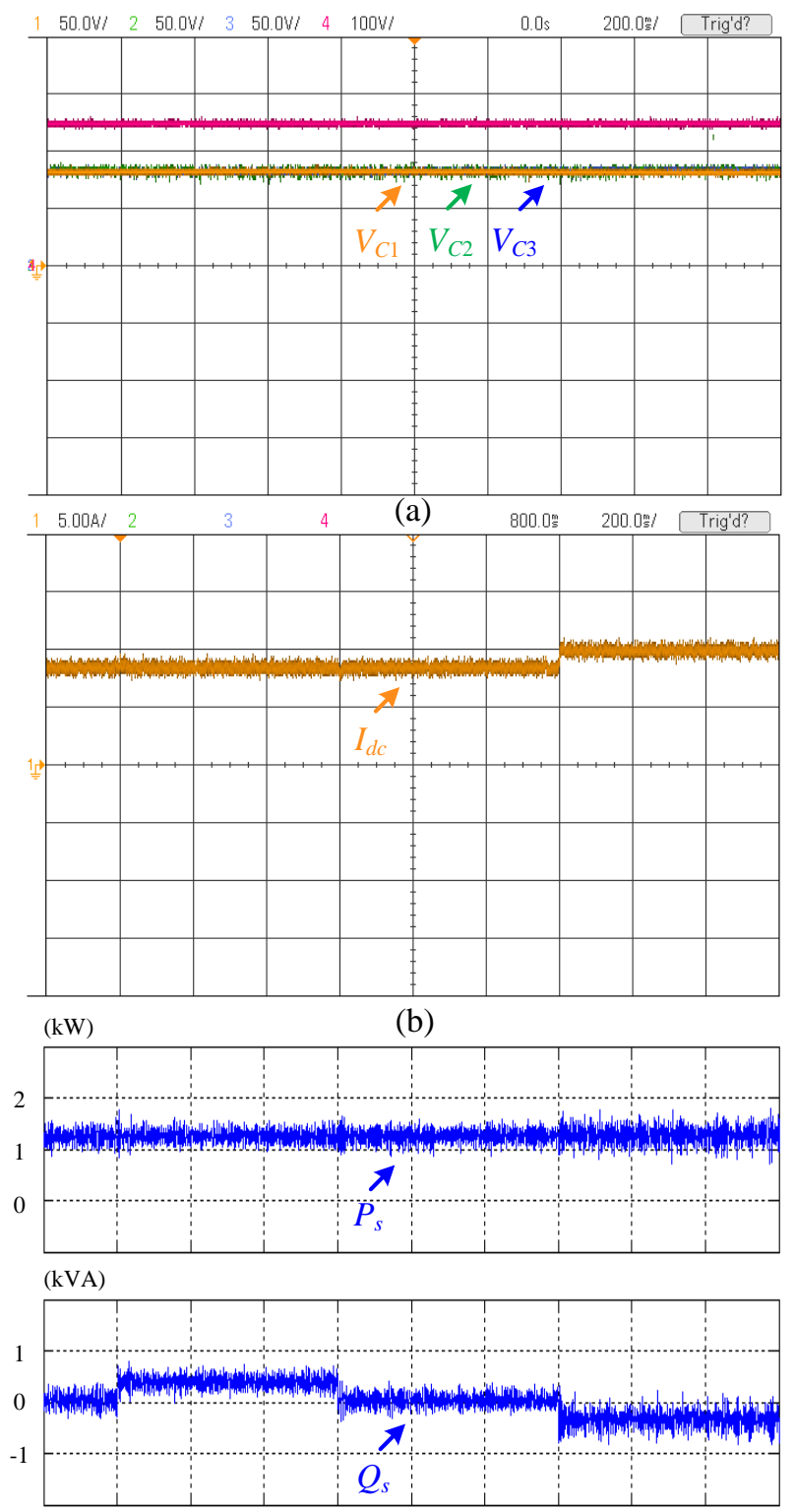

(c)

Figure 2-33 Experimental performance under reactive power control.

(c) Performance under reactive power control

Figure 2-33 illustrates the experimental performance of the converter for the proposed configuration under reactive power control. Different reactive power $Q_{s}(0 \mathrm{pu}, 0.33 \mathrm{pu},-0.33 \mathrm{pu})$ are separately injected into the local grid. As in Figure 2-33 (b), a minimum dc-link current $I_{d c}$ is changed accordingly to minimize system loss. The input capacitor voltages $V_{c 1}, V_{c 2}$, and $V_{c 3}$ shown in Figure 2-33 (c) are distributed evenly during all steady and transient states. 


\subsubsection{Experimental verification for $L V$ configuration}

Similarly, an extra resistor is connected across the output of Module 3 to introduce current imbalance on purpose.

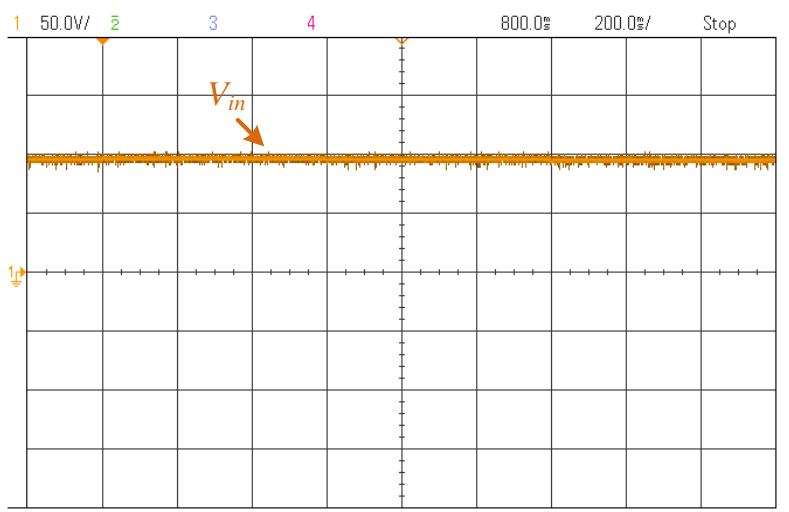

(a)

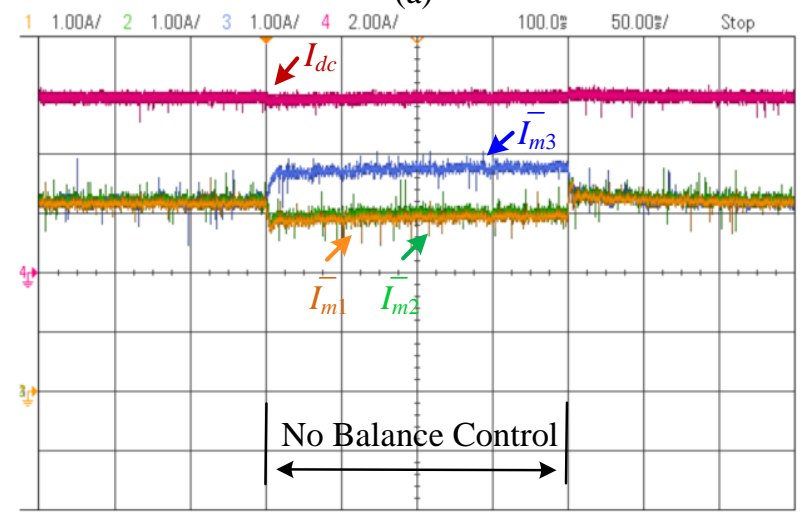

(b)

Figure 2-34 Experimental performance with/without average current sharing control.

(a) Performance with and without current balance control

Figure 2-34 shows the experimental performance with/without current balance control. $V_{\text {in }}$ is the input voltage of the modular converter; $\bar{I}_{m 1,2,3}$ are the measured average input currents for Module 1, 2, and 3, respectively; and $I_{d c}$ is the measured dc-link current. First, the system is operating under conditions of $V_{i n}=100 \mathrm{~V}$ and $I_{d c}=6 \mathrm{~A}$. With current sharing control, the average input currents are balanced with $\bar{I}_{m 1}=\bar{I}_{m 2}=\bar{I}_{m 3}$ as shown in Figure 2-34 (b). At time $t$, the current balance scheme is deactivated. The average input currents of the three modules start to diverge quickly due to the mismatch among the constituent modules. Module 3 draws the highest current due to the extra resistor. Finally, the input currents quickly converge to their normal values when the current balance control is reactivated. 


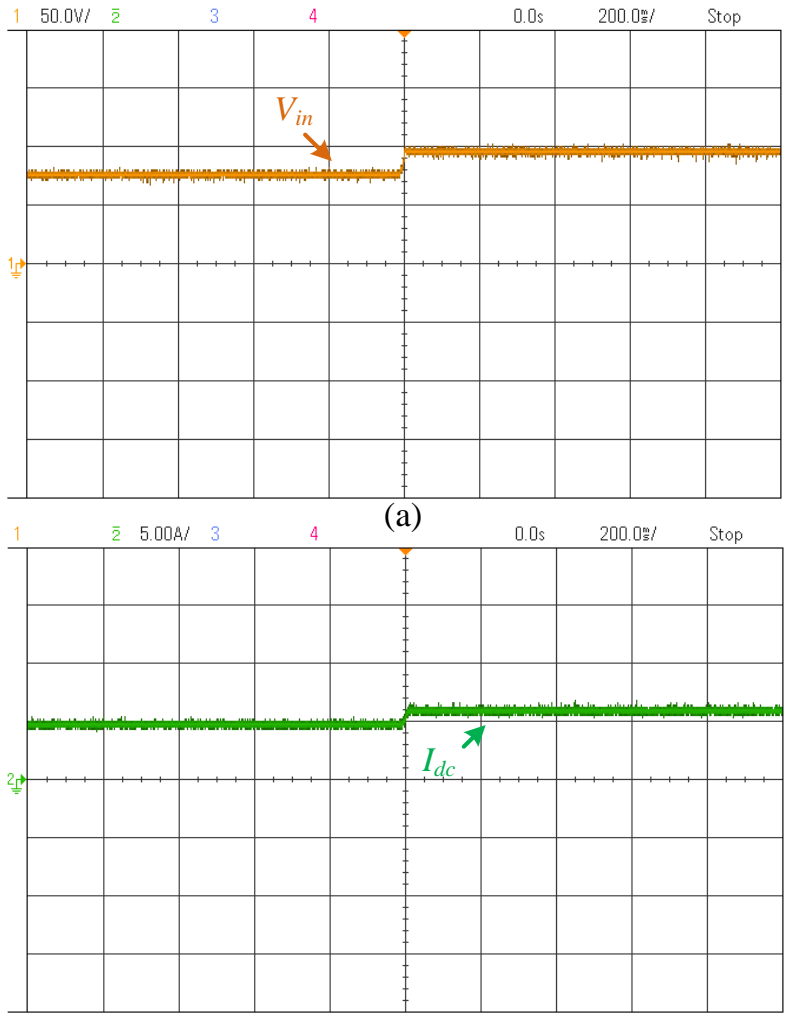

(b)

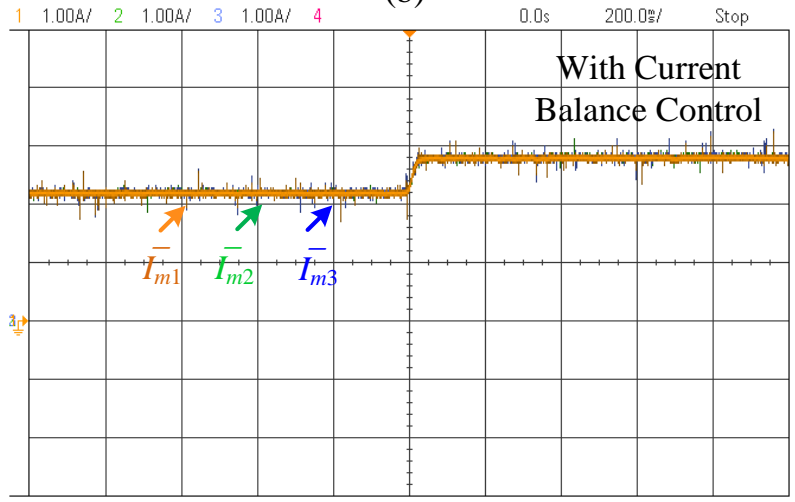

(c)

Figure 2-35 Experimental performance under stepped input voltage.

(b) Performance under stepped wind speed

Figure 2-35 illustrates the experimental performance under stepped input voltage from $0.8 \mathrm{pu}$ $(80 \mathrm{~V})$ to $1 \mathrm{pu}(100 \mathrm{~V})$. The resultant dc-link current $I_{d c}$ increases from 5 A to $6 \mathrm{~A}$. During the operation process, the average input currents are well balanced as shown in Figure 2-35 (c). 


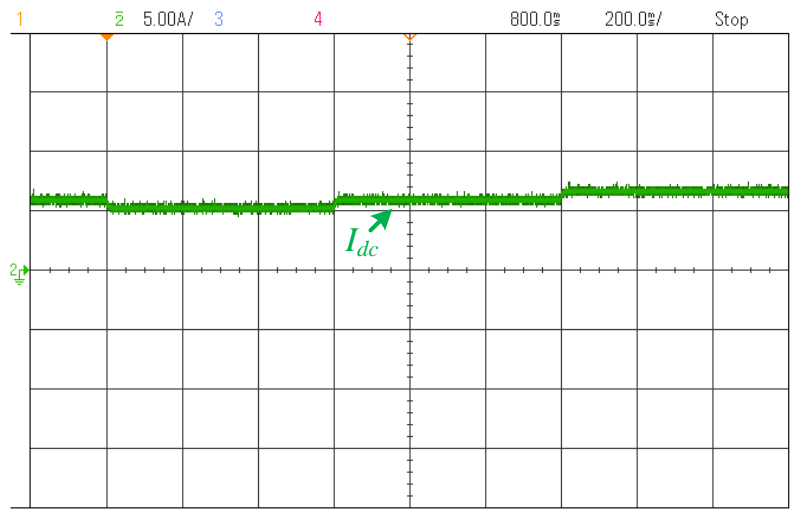

(a)

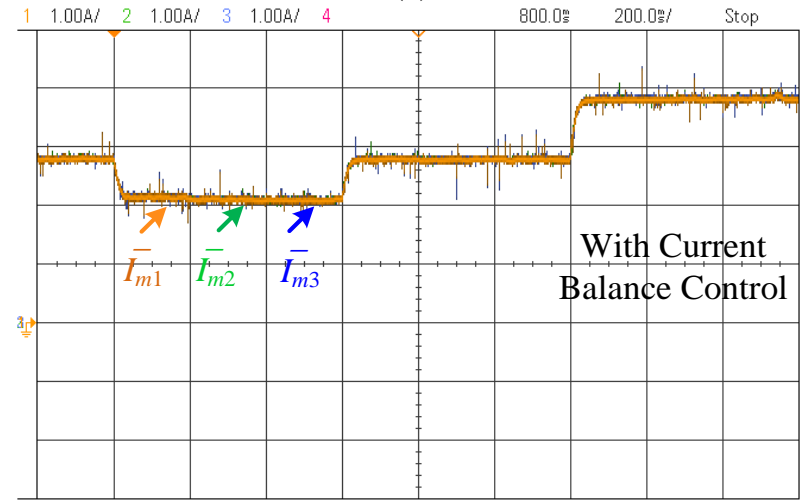

(b)
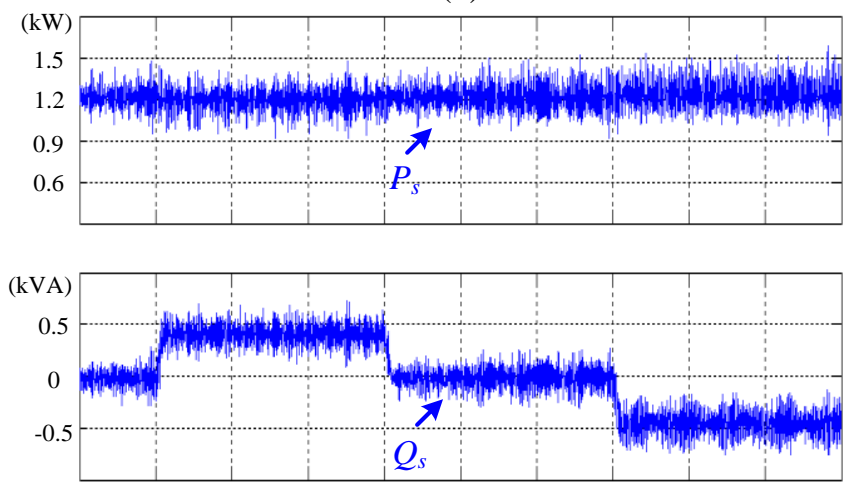

(c)

Figure 2-36 Experimental performance under stepped reactive power control.

(c) Performance under reactive power control

Figure 2-36 illustrates the experimental performance under reactive power control. Different reactive power $Q_{s}(0 \mathrm{pu}, 0.33 \mathrm{pu},-0.33 \mathrm{pu})$ shown in Figure 2-36 (c) are separately injected into the local grid. As shown in Figure 2-36 (a), a minimum dc-link current $I_{d c}$ is changed accordingly to minimize system loss. The average input currents of the modular converter shown in Figure 2-36 (b) are well balanced during both steady and transient states. 


\subsection{Conclusions}

In this chapter, modular medium-frequency transformer (MFT)-based configurations are proposed for medium-voltage (MV) and low-voltage (LV) turbine-based offshore wind farms, respectively. Compared with commercial configurations, the proposed ones give significant cost saving thanks to the series-connected structure where the very bulky and costly offshore substation is eliminated. Compared with existing series-connected configurations, the proposed ones feature smaller size and weight and higher reliability thanks to the adaption of the modular MFTs-based converter.

The modular MFT-based converter used in MV configuration is connected in series at both input and output, while it is in parallel at input and in series at output for LV configuration. In practice, tolerance of the components of the modular converter exists, leading to uneven distribution of power among the constituent modules. To solve this issue, a voltage balance control scheme is proposed for MV configuration and a current balance control is proposed for the LV one.

In addition, the performance of the proposed system considering the effects of cables are investigated. The possible maximum peak value of the dc-link voltage is two times of its rated value, while the dc-link current is not affected. To avoid the two-time rated dc-link voltage, a dc filter is introduced to each offshore converter. The design of the dc filter and dc-link inductor are given.

Both simulation and lab-scale experiments are conducted to verify the performance of the proposed configurations and the effectiveness of the proposed schemes. 


\section{Chapter 3}

\section{Natural Sampling SVM with Superior}

\section{Harmonics Performance for CSCs}

In the proposed configuration, MV CSCs are used as grid-side converters. MV CSCs are well proven converters in high-power MV drives [22]. In practice, only selective harmonics elimination (SHE) is used for MV CSCs. SHE features superior low-order harmonics performance, but it is an off-line modulation scheme with slow dynamic response. Space vector modulation (SVM) featuring fast dynamic response and being the proper scheme to achieve common mode voltage reduction, active damping, and power factor control, however, cannot be used in practice mainly due to its poor low-order harmonics performance. Therefore, the objective of this chapter is to propose an advanced SVM scheme for grid-side MV CSC with superior harmonics performance.

This chapter starts with a brief overview of existing modulation schemes for MV CSC, followed by the introduction of the proposed natural sampling SVM (NS-SVM). Compared with conventional SVM, NS-SVM preserves low switching/sampling frequency and high dynamic performance, but features superior low-order harmonics performance that can be comparable to SHE. Besides, an optimal space vector sequence in investigated based on NS-SVM and the best one in terms of low-order harmonics performance is selected. Experimental results are provided to verify the effectiveness of the proposed NS-SVM.

\subsection{Existing modulation schemes for CSCs}

Figure 3-1 shows the topology of the grid-side CSC in the proposed system. The used switching devices are symmetrical gate-commutated thyristors (SGCT) with reverse voltage blocking capability. The device switching frequency is normally limited to approximately 500 $\mathrm{Hz}$ to reduce switching loss and satisfy the stringent thermal requirements of devices. Besides, a CSC normally requires a three-phase capacitor $C_{f}$ at its output to assist the commutation of the switching devices and filter the output PWM current. 


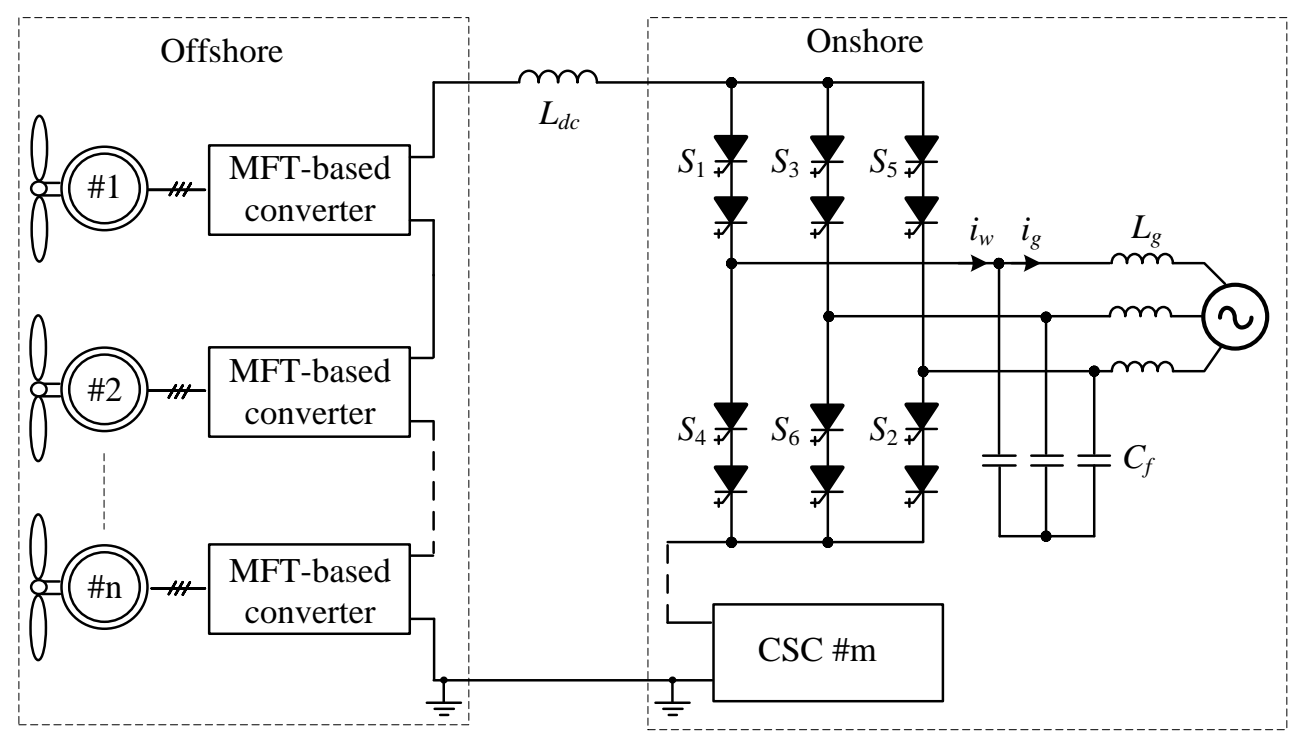

Figure 3-1 Topology of grid-side CSC.

The switching pattern design for a CSC should generally satisfy: at any instant of time (excluding commutation intervals) there are only two switches conducting, one in the top half of the bridge and the other in the bottom. Generally, the major modulation schemes for MV CSCs are SHE, trapezoidal pulse width modulation (TPWM), and SVM [22].

SHE features superior low-order harmonics performance that it can eliminate a number of loworder unwanted harmonics with low switching frequency. However, SHE is an offline modulation scheme lacking the flexibility for control. In practice where variation of the modulation index is needed [52], the solutions for all the required modulation indices have to be calculated offline and stored in the system prior to operation, which is impractical. TPWM is a carrier based modulation scheme. Although it allows the modulation index to be changed from 0 to 1 , the dc utilization rate is restricted to between 0.66 and 0.74 . Thus, the controllable range of output current magnitude is quite limited. Compared with SHE and TPWM, SVM provides higher dynamic performance since (a) its modulation index can be adjusted within a sampling period and (b) the CSC output PWM current $i_{w}$ can be directly controlled by the bypass operation instead of dc-link current adjustment by the front-end converter. However, SVM is not used in grid-side MV CSC. This is because its output contains high-magnitude low-order harmonics, particularly the fifth and seventh harmonics, lying closely to the resonance frequency of the LC filter of the converter, and will introduce resonance as the grid-side damping is insufficient [22]. 
Previous research on SVM of CSCs is mainly about mapping from VSC vectors to CSC vectors [53]-[56], and direct SVM of a CSC with different vector sequences [57]-[60]. Unfortunately, few studies have focused on reducing low-order harmonics of SVM. Ref. [61] investigated the magnitudes of lower-order harmonics (5th and 7th) of different vector sequences and combined two of these sequences to give a better harmonic performance. But this approach adds complexity to the implementation and the overall performance is still not desirable. Refs. [62]-[64] proposed new SVM schemes to reduce the magnitude of low-order harmonics by repositioning the reference vectors in each counter period. However, the improvement is limited at high modulation indices. Ref. [65] introduced a multisampling SVM scheme. It features good low-order harmonics performance but with penalties of higher sampling frequency and additional switching frequency. In addition, the harmonics performance highly relies on the sampling frequency that higher sampling frequency is required to achieve better performance.

With special focus on achieving superior low-order harmonics performance while preserving low switching/sampling frequency and high dynamic performance of conventional SVM, a NSSVM is proposed for the grid-side CSC.

\subsection{Natural sampling SVM}

A CSC has a total of nine switching states as shown in Table 3-1. A typical space vector diagram for the CSC is shown in Figure 3-2, where $\vec{I}_{1}$ to $\overrightarrow{\boldsymbol{I}}_{6}$ are active vectors and $\overrightarrow{\boldsymbol{I}}_{\mathrm{o}}$ is the zero vector. The active vectors form a regular hexagon with six equal sectors, while the zero vector $\overrightarrow{\boldsymbol{I}}_{\mathrm{o}}$ lies on the center of the hexagon. The current reference vector $I_{\text {ref }}$ in Figure 3-2 (a) rotates in space at an angular velocity, $\omega=2 \pi f_{1}$, where $f_{1}$ is the fundamental frequency of the inverter output current $i_{w}$. The length of the reference $I_{r e f}$ represents the magnitude of the output current, which is determined by the modulation index $m_{a}$ of the converter, given as $I_{r e f} / I_{d c} \cdot \theta$ is the angular displacement between the current reference vector and $\alpha$ axis. For a given length and position, $I_{r e f}$ can be synthesized by the three nearby vectors. For example, with $I_{r e f}$ falling into sector I (Figure 3-2 (b)), Iref can be synthesized by $\vec{I}_{1}, \vec{I}_{2}$ and $\overrightarrow{\boldsymbol{I}}_{\mathrm{o}}$ [22]. The dwell times for the vectors can be calculated based on the ampere-second balancing principle.

The resultant dwell times are expressed as: 


$$
\begin{gathered}
T_{1}=m_{a} \sin (\pi / 6-\theta) T_{s} \text { for }-\pi / 6 \leq \theta \leq \pi / 6 \\
T_{2}=m_{a} \sin (\pi / 6+\theta) T_{s} \text { for }-\pi / 6 \leq \theta \leq \pi / 6 \\
T_{0}=T_{s}-T_{1}-T_{2} \text { for }-\pi / 6 \leq \theta \leq \pi / 6
\end{gathered}
$$

where $T_{1}, T_{2}$, and $T_{0}$ are the dwell times for the vectors $\overrightarrow{\boldsymbol{I}}_{1}, \overrightarrow{\boldsymbol{I}}_{2}$, and $\overrightarrow{\boldsymbol{I}}_{\mathrm{o}}$. Note that (3-1), (3-2), and (3-3) can also be used by rearranging the actual angle $\theta$ when $I_{r e f}$ is in other sectors. The modified angle $\theta^{\prime}$ falls into sector I:

$$
\theta^{\prime}=\theta-(k-1) \pi / 3 \text { for }-\pi / 6 \leq \theta^{\prime} \leq \pi / 6
$$

where $k=1,2 \ldots 6$ for sectors I, II... VI, respectively.

Table 3-1 Switching States and Space Vectors

\begin{tabular}{|c|c|c|c|}
\hline Type & Switching States & On-State Switch & Space Vector \\
\hline \multirow{3}{*}{$\begin{array}{c}\text { Zero } \\
\text { States }\end{array}$} & {$[14]$} & $S_{1}, S_{4}$ & \multirow{2}{*}{$\vec{I}_{\mathrm{o}}$} \\
\cline { 2 - 3 } & {$[36]$} & $S_{3}, S_{6}$ & \\
\hline \multirow{4}{*}{ Active } & {$[52]$} & $S_{5}, S_{2}$ & \\
\cline { 2 - 4 } States & {$[61]$} & $S_{6}, S_{1}$ & $\vec{I}_{1}$ \\
\cline { 2 - 4 } & {$[12]$} & $S_{1}, S_{2}$ & $\vec{I}_{2}$ \\
\cline { 2 - 4 } & {$[23]$} & $S_{2}, S_{3}$ & $\vec{I}_{3}$ \\
\cline { 2 - 4 } & {$[34]$} & $S_{3}, S_{4}$ & $\vec{I}_{4}$ \\
\cline { 2 - 4 } & {$[45]$} & $S_{4}, S_{5}$ & $\vec{I}_{5}$ \\
\hline
\end{tabular}

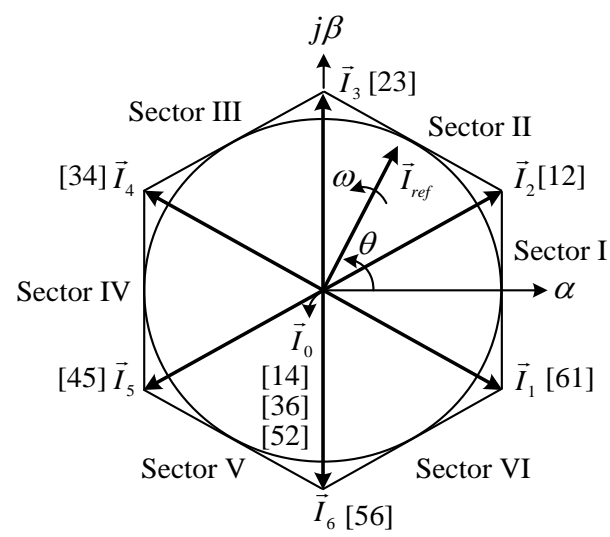

(a) Space vector diagram

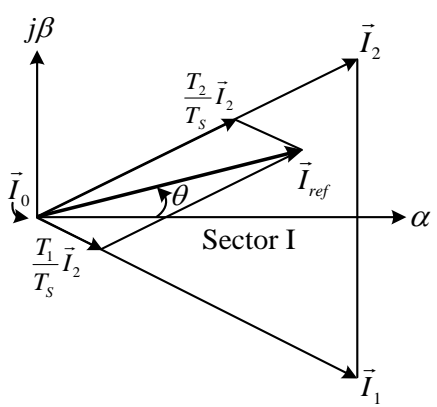

(b) Vector synthesis in one sector

Figure 3-2 Operating principle of SVM for CSCs.

\subsubsection{Conventional SVM}

Note that The following analysis are conducted under the typical conditions of switching 
frequency $\left(f_{s w}\right)=540 \mathrm{~Hz}$ and sampling frequency $\left(f_{s p}\right)=1080 \mathrm{~Hz}$.

In the case of conventional SVM, the dwell time for each selected vector is calculated once at the beginning of each sampling period (carrier period) and kept fixed within one sample period. The dwell time for the first vector $\vec{I}_{n}$ of the three-segment sequence $\left(\vec{I}_{n} \vec{I}_{n+1} \vec{I}_{\mathrm{o}}\right)$ in Sector I is taken as an example to illustrate the principle of conventional SVM.

As shown in Figure 3-3, the blue lines $\left(T_{1}(\mathrm{Con}-\mathrm{SVM})\right)$ are the dwell times for the first vector $\vec{I}_{n}$ calculated based on conventional SVM (3-1):

$$
\begin{array}{ll}
T_{1}=\sin (\pi / 6-(-\pi / 6)) T_{s} & \text { for }-\pi / 6 \leq \theta \leq \pi / 18 \\
T_{1}=\sin (\pi / 6-(-\pi / 18)) T_{s} & \text { for }-\pi / 18 \leq \theta \leq \pi / 18 \\
T_{1}=\sin (\pi / 6-(\pi / 18)) T_{s} & \text { for } \pi / 18 \leq \theta \leq \pi / 6
\end{array}
$$

The red line ( $\left.T_{1}(\mathrm{NS}-\mathrm{SVM})\right)$ shown in Figure 3-3 refers to the continuous dwell time (3-1). Obviously, discretization error exists during the digital implementation of conventional SVM. Such a discretization error causes high-magnitude low-order harmonics [65], especially the 5th and 7th harmonics, lying closely to the resonance frequency of LC filter (3.5-5.5pu) of the converter, and will introduce resonance as the line damping is insufficient [66]. Therefore, conventional SVM is not used in commercial high-power MV CSC-based drives [22].

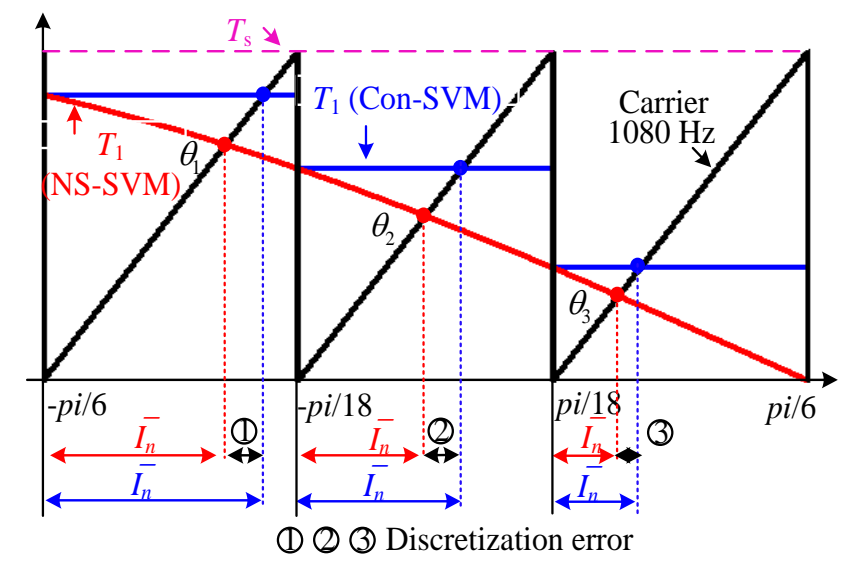

Figure 3-3 Dwell time calculation of conventional SVM and NS-SVM.

\subsubsection{Natural sampling SVM}

The discretization error for conventional SVM exists in digitally implemented control because of the limited sampling frequency. On the other hand, the low-order harmonics can be significantly reduced if the discretization error can be eliminated (a natural sampling 
performance as that in analog control). Two methods can help, one is by infinite sampling frequency, and the other is by the proposed NS-SVM. The former is impractical, while the latter (NS-SVM) can effectively and efficiently achieve that [67].

As shown in Figure 3-3, $\theta_{1}, \theta_{2}, \theta_{3}$ are natural sampling-based dwell times. It implies that a naturally sampled performance as same as that in analog control can be achieved based on $\theta_{1}, \theta_{2}, \theta_{3}$. Then, the question goes to how to find $\theta_{1}, \theta_{2}, \theta_{3}$. Figure 3-3 shows that $\theta_{1}, \theta_{2}, \theta_{3}$ are intersections of the vector reference and the carrier. The vector reference $T_{1}$ is shown in (3-1). The equation of the carrier can be derived as follows:

(a) number of carrier pulse determination. For example, three are three carrier pulses in each sector under $f_{s w}=540 \mathrm{~Hz}$; and

(b) derivation of the linear carrier. For example, the equation for the first carrier can be derived based on points $(-\pi / 6,0)$ and $\left(-\pi / 18, T_{s}\right)$.

The resultant equations for the carriers are expressed as

$$
\begin{array}{ll}
f(\theta)=(9 \theta / \pi+3 / 2) T_{s} & \text { for }-\pi / 6 \leq \theta \leq-\pi / 18 \\
f(\theta)=(9 \theta / \pi+1 / 2) T_{s} & \text { for }-\pi / 18 \leq \theta \leq \pi / 18 \\
f(\theta)=(9 \theta / \pi-1 / 2) T_{s} & \text { for } \pi / 18 \leq \theta \leq \pi / 6
\end{array}
$$

By combining (3-1) and (3-6), the natural sampling-based dwell times can be solved. However, these equations are nonlinear that are difficult to get online. To solve this issue, simplified but precise linear dwell time equations are derived based on Newton-Raphson algorithm [22].

$\boldsymbol{\theta}_{1}$ is taken as an example to illustrate how to derive the linear equation.

The solving equation $(F(\theta))$ for $\theta_{1}$ is obtained by combining (3-1) and (3-6):

$$
F(\theta)=m_{a} \sin (\pi / 6-\theta) T_{s}-(9 \theta / \pi+3 / 2) T_{s}
$$

The initial guess of $\theta_{1}$ is represented by $\theta_{10}$ :

$$
\theta_{10}=-\pi / 6
$$

The result after one iteration $\theta_{11}$ : 


$$
\theta_{11}=\theta_{10}-\frac{F\left(\theta_{10}\right)}{\left.F^{\prime}(\theta)\right|_{\theta=\theta_{10}}}=\theta_{10}+\frac{m_{a} \sin \left(\pi / 6-\theta_{10}\right)-\left(9 \theta_{10} / \pi+3 / 2\right)}{m_{a} \cos \left(\pi / 6-\theta_{10}\right)+9 / \pi}
$$

The result after the second iteration $\theta_{12}$ :

$$
\theta_{12}=\theta_{11}-\frac{F\left(\theta_{11}\right)}{\left.F^{\prime}(\theta)\right|_{\theta=\theta_{11}}}=\theta_{11}+\frac{m_{a} \sin \left(\pi / 6-\theta_{11}\right)-\left(9 \theta_{11} / \pi+3 / 2\right)}{m_{a} \cos \left(\pi / 6-\theta_{11}\right)+9 / \pi}
$$

The switching angle $\boldsymbol{\theta}_{1}$ calculated after two iterations (3-9) and (3-10), is precise enough in terms of harmonics performance compared with that of the original set of equations. The other switching angles can be achieved with the same manner, thus not repeated here. A flow chart is provided in Figure 3-4 where $\boldsymbol{\theta}_{1}$ is taken an example to illustrate the derivation principle.

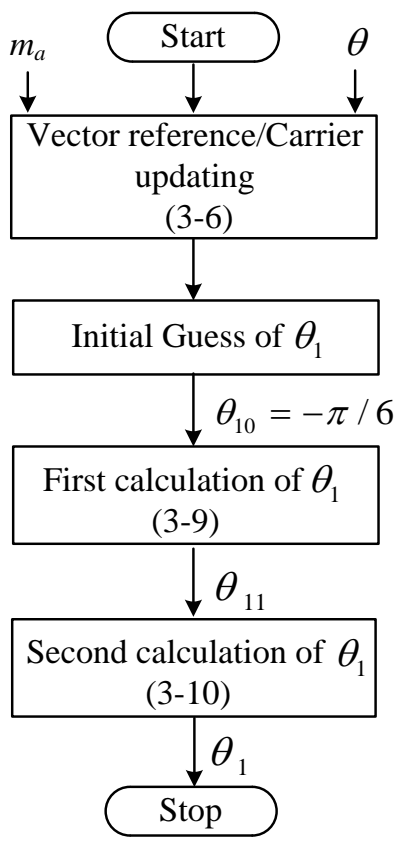

Figure 3-4 Flow chart of dwell times solving based on two-step iterations.

In summary, the proposed NS-SVM is able to find the natural sampling-based dwell times online based on the derived two-step equations. What is more, the used switching/sampling frequency is maintained same as that in conventional SVM.

\subsubsection{Comparison between conventional SVM and NS-SVM}

The comparison of low-order harmonics performance between conventional SVM and the proposed NS-SVM is carried out based on same conditions, that are same switching/sampling 
frequency $(540 / 1080 \mathrm{~Hz})$ and same space vector sequence (three-segment sequence).

The harmonic profile of conventional SVM is given in Figure 3-5. $I_{w n}$ is the rms value of the nth-order harmonic current in $i_{w}$ and $I_{w 1, \max }$ is the maximum rms fundamental-frequency current under the condition of $m_{a}=1$. The 5th and 7 th harmonics are $10.36 \%$ and $7.8 \%$ of the fundamental component, respectively. The harmonic profile of NS-SVM is illustrated in Figure 3-6 where the 5th and 7th harmonics are reduced to almost zero. By contrast, the high-order harmonics (17th, 19th, 23th, and 25th harmonics) are slightly higher than those of a conventional SVM. However, this is not an issue because these high-order harmonics can be effectively damped by the LC filter.

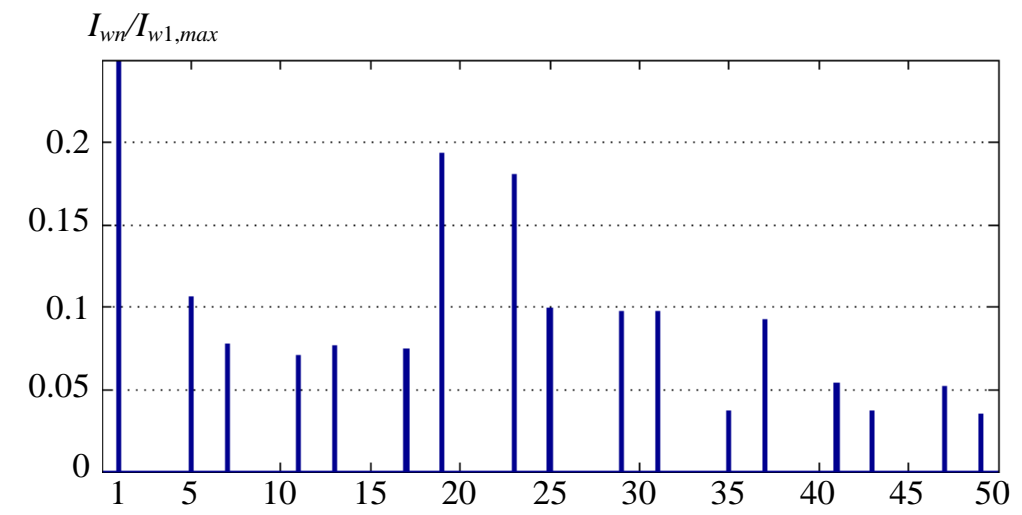

Figure 3-5 Harmonic profile of output PWM current based on conventional SVM.

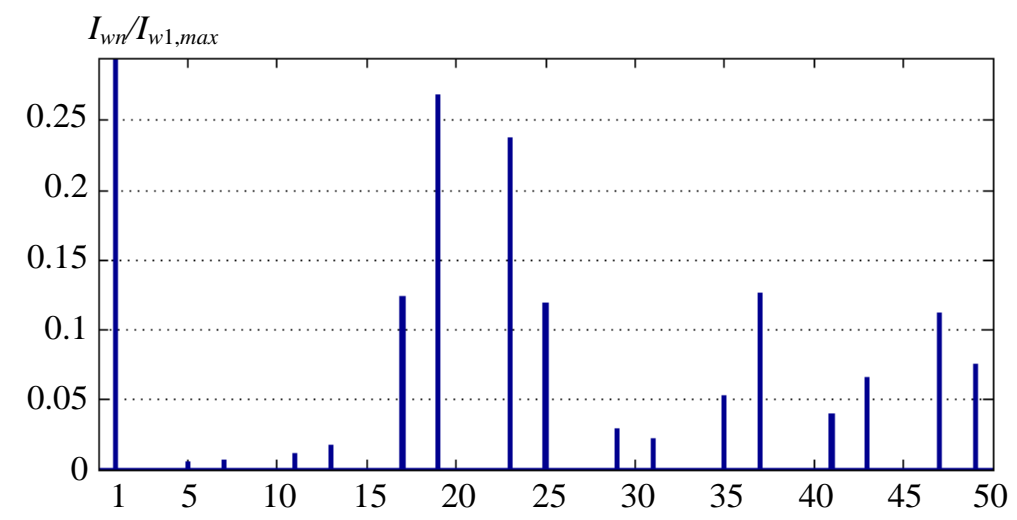

Figure 3-6 Harmonic profile of output PWM current based on NS-SVM.

The main low-order harmonics $\left(5^{\text {th }}, 7^{\text {th }}, 11^{\text {th }}\right.$, and $\left.13^{\text {th }}\right)$ in the full operation range based on conventional and the proposed NS-SVM are presented in Figure 3-7. Figure 3-7 shows that the 
proposed NS-SVM has better low-order harmonics (5th, 7th, 11th, and 13th) performance in the full operation range, especially at high modulation indices.
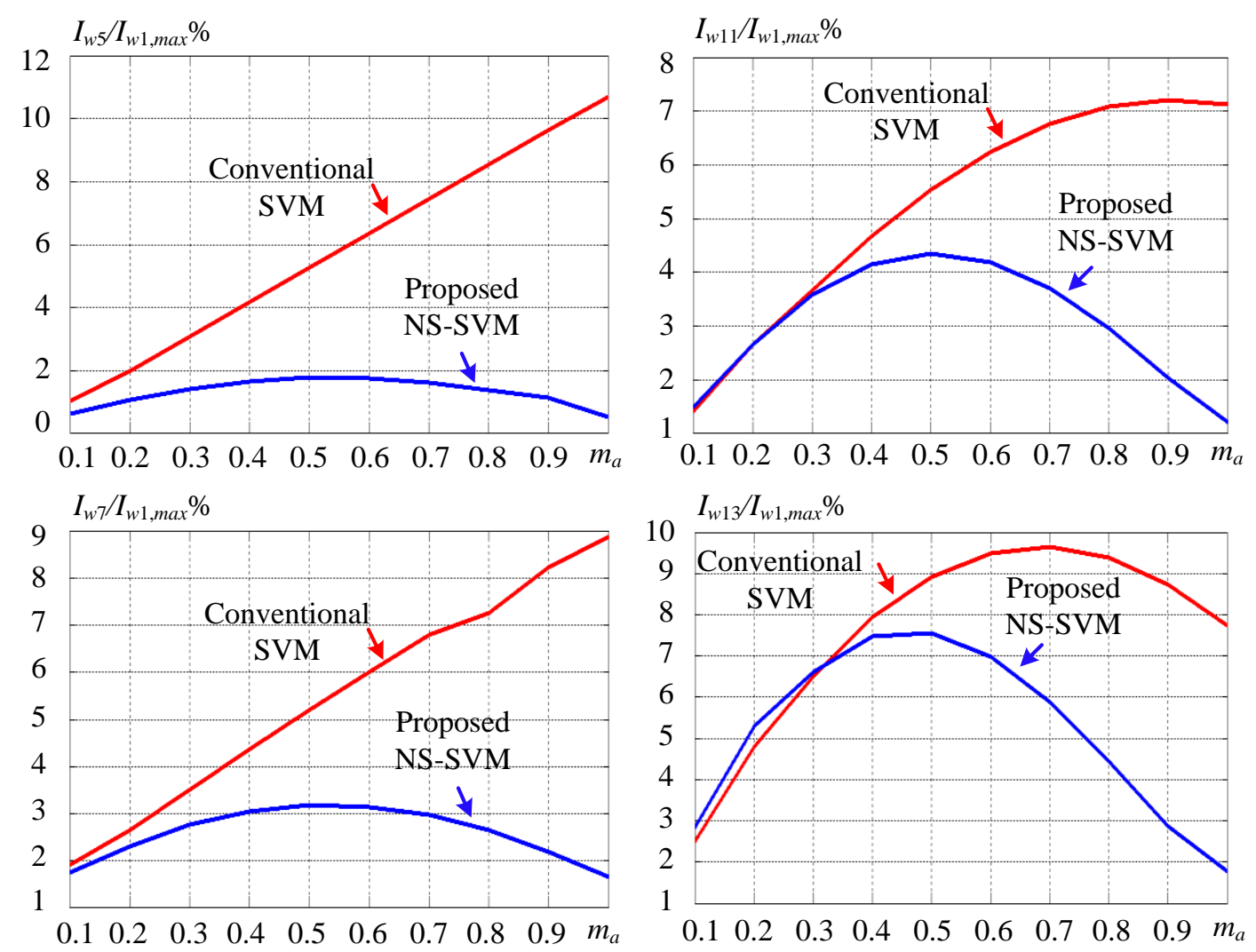

Figure 3-7 Low-order harmonics performance between conventional SVM and NS-SVM.

In summary, compared with conventional SVM, the proposed NS-SVM gives a superior loworder harmonics performance in the full operation range without compromising performance in terms of low switching/sampling frequency and high dynamic performance.

\subsection{Optimal space vector sequence design}

Ref. [61] investigated the performance of low-order harmonics (5th and 7th harmonics) of different vector sequences based on conventional SVM and concluded that the harmonic performance can be improved by combining two sequences under proper modulation index and different LC filters lead to different combinations. However, the harmonics performance in [61] is still unsuitable for practical applications. Besides, its implementation is complex as the switching between two space vector sequences at different modulation indices and LC filters is required. 
With a special focus on low-order harmonics, this section investigates the low-order harmonics performance of different space vector sequences based on the proposed NS-SVM. The sequence with the best harmonics performance will be selected [68].

\subsubsection{Implementation of different sequences}

The most discussed space vector sequences for a CSC in the literature [55]-[60] are shown in Figure 3-8. SQ1-SQ3 is generated by using the saw tooth carrier, while SQ4-SQ6 is realized with triangle carrier with the asymmetrical sampled PWM.

(a) SQ1

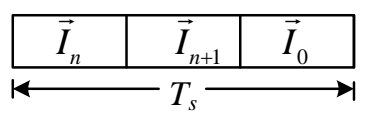

(b) SQ2

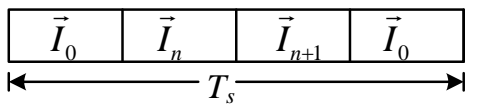

(c) SQ3

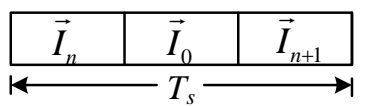

(d) SQ4

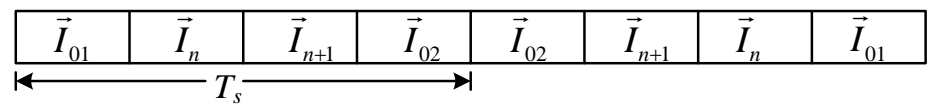

(e) SQ5

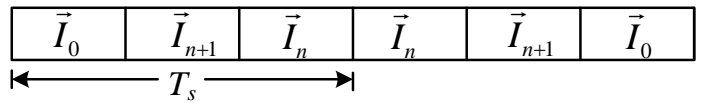

(f) SQ6

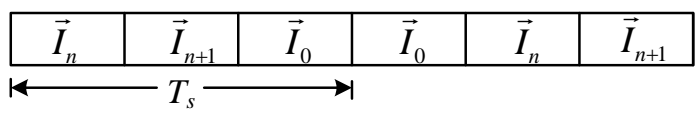

Figure 3-8 Different space vector sequences.

To make a fair comparison, a couple of constraints and requirements are specified in advance. First, the switching sequence design for the CSC should satisfy two requirements for the minimization of switching frequency: (a) the transition from one switching state to the next involves only two switches, one being switched on and the other switched off; and (b) the transition for the combined current reference moving from one sector to the next requires the minimum number of switches. Second, three-phase symmetry (to eliminate unbalancing effects) and half-wave symmetry (to eliminate even-order harmonics) need to be satisfied. The quarterwave symmetry does not offer obvious harmonic performance advantage over the half-wave symmetry and, thus, is not considered [61]. Third, the switching frequency is limited to around $500 \mathrm{~Hz}$ to reduce switching loss and satisfy the stringent thermal requirements of devices [22]. Note that, with consideration of the above constraints and requirements, the switching frequency 
of different sequences might not be same [61]. In summary, the present work aims at finding the most suitable space vector sequences for MV CSCs based on NS-SVM.

Case A: Sequence 1

Space vector sequence 1 (SQ1) shown in Figure 3-8 (a) is the traditional three-segment sequence. Figure 3-9 shows that in each sector, there are six dwell times. The dwell time references are $T_{1}$ (3-1) and $T_{1}+T_{2}$. The carriers are same with that in (3-6), thus not repeated here. Combining (3-1) and (3-6) gives the solutions of $\theta_{1}, \theta_{3}, \theta_{5}$. Similarly, $\theta_{2}, \theta_{4}, \theta_{6}$ is obtained by the combination of (3-11) and (3-6).

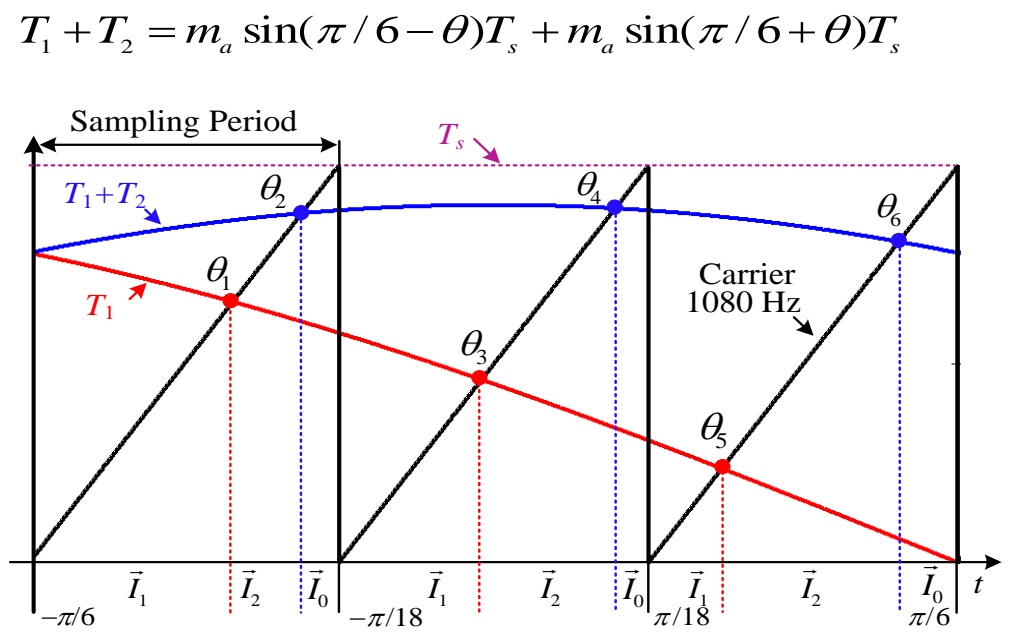

Figure 3-9 Sequence 1 (Sector I).

Case B: Sequence 2

Space vector sequence 2 (SQ2) shown in Figure 3-8 (b) divides the zero vector and puts them at the start and the end of the sequence. Figure 3-10 shows the details of SQ2 in Sector I. To achieve a minimum device switching frequency, all the zero vectors in one sector should be same except the last one. The last one should be same as the first one selected in the beginning of the next sector. On this basis, during the transition from one sector to the next only two switches are involved. Otherwise, four switches will be generated if the two zero vectors are different. A switching frequency of $540 \mathrm{~Hz}$ and a sampling frequency of $1080 \mathrm{~Hz}$ are therefore selected.

As shown in Figure 3-10, there are nine dwell times $\theta_{1}, \theta_{2}, \theta_{3}, \theta_{4}, \theta_{5}, \theta_{6}, \theta_{7}, \theta_{8}, \theta_{9}$ for SQ2 per sector. The carriers are same with that of SQ1, but the dwell time references are different. The three dwell time references are $T_{0} / 2, T_{1}+T_{0} / 2$ and $T_{1}+T_{2}+T_{0} / 2$. As same as that in SQ1, the 
combination of carriers and references gives the solution of the dwell times.

$$
\begin{gathered}
T_{0} / 2=\left(T_{s}-m_{a} \sin (\pi / 6-\theta) T_{s}-m_{a} \sin (\pi / 6+\theta) T_{s}\right) / 2 \\
T_{1}+T_{0} / 2=\left(T_{s}+m_{a} \sin (\pi / 6-\theta) T_{s}-m_{a} \sin (\pi / 6+\theta) T_{s}\right) / 2 \\
T_{1}+T_{2}+T_{0} / 2=\left(T_{s}+m_{a} \sin (\pi / 6-\theta) T_{s}+m_{a} \sin (\pi / 6+\theta) T_{s}\right) / 2
\end{gathered}
$$

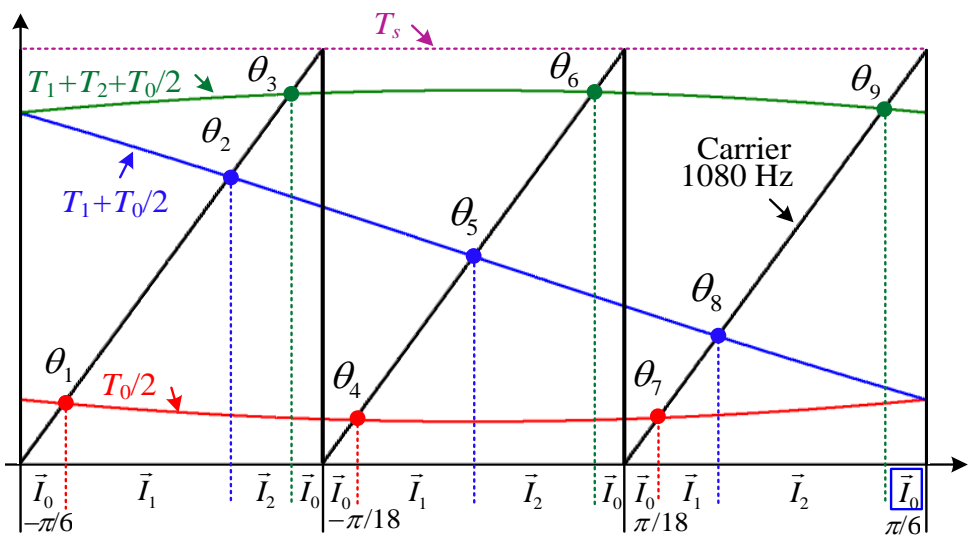

Figure 3-10 Sequence 2 (Sector I).

\section{Case C: Sequence 3}

Space vector sequence 3 (SQ3) shown in Figure 3-8 (c) puts the zero vector in the center. The last active vector in one sector is same as the first active vector selected in the beginning of next sector. Thus, the switching frequency is reduced from $540 \mathrm{~Hz}$ to $480 \mathrm{~Hz}$. SQ3 has six dwell times $\theta_{1}, \theta_{2}, \theta_{3}, \theta_{4}, \theta_{5}, \theta_{6}$ per sector. The carrier equations are same as that in SQ1 and SQ2. The dwell time references are $T_{1}(3-1)$ and $T_{1}+T_{0}$.

$$
T_{1}+T_{0}=T_{s}-m_{a} \sin (\pi / 6+\theta) T_{s}
$$

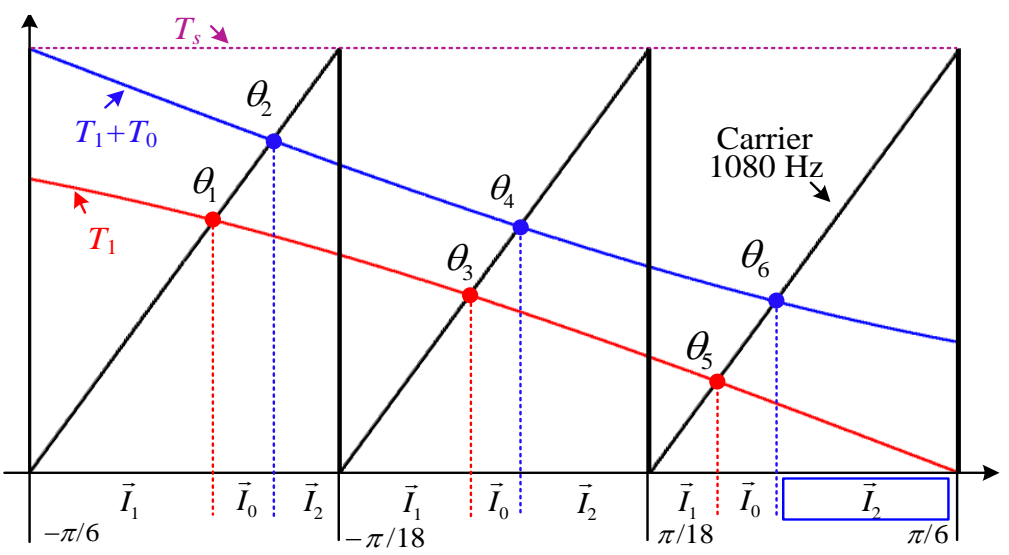

Figure 3-11 Sequence 3 (Sector I). 


\section{Case D: Sequence 4}

Space vector sequence 4 (SQ4) shown in Figure 3-8 (d) is generated by the triangle carrier waveform with the asymmetrical sampled PWM (dwell times are calculated every half-carrier period). Again, to maintain waveform symmetry, the sequence should restart at each sector boundary (instead of continue), and a proper zero vector should be selected at a sector boundary; as same in SQ2, the zero vector (highlighted by a blue block) in the last sample in the present sector should be selected as the zero vector (highlighted by a red block) for the next sector. A $540 \mathrm{~Hz}$ switching frequency and a $1080 \mathrm{~Hz}$ sampling frequency are suitable [61] . The resultant carriers for SQ4 are therefore divided into two parts.

In Sectors I, III, and V (Figure 3-12), the carrier equations are:

$$
\begin{aligned}
& f(\theta)=(9 \theta / \pi+3 / 2) T_{s} \text { for }-\pi / 6 \leq \theta \leq-\pi / 18 \\
& f(\theta)=(-9 \theta / \pi+1 / 2) T_{s} \text { for }-\pi / 18 \leq \theta \leq \pi / 18 \\
& f(\theta)=(9 \theta / \pi-1 / 2) T_{s} \text { for } \pi / 18 \leq \theta \leq \pi / 6
\end{aligned}
$$

The corresponding dwell time references are $T_{0} / 2, T_{1}+T_{0} / 2$, and $T_{1}+T_{2}+T_{0} / 2$.

In Sectors II, IV, and VI (Figure 3-12), the carrier equations are expressed as:

$$
\begin{aligned}
& f(\theta)=(-9 \theta / \pi-1 / 2) T_{s} \text { for }-\pi / 6 \leq \theta \leq-\pi / 18 \\
& f(\theta)=(9 \theta / \pi+1 / 2) T_{s} \text { for }-\pi / 18 \leq \theta \leq \pi / 18 \\
& f(\theta)=(-9 \theta / \pi+3 / 2) T_{s} \text { for } \pi / 18 \leq \theta \leq \pi / 6
\end{aligned}
$$

The corresponding dwell time equations are $T_{0} / 2, T_{2}+T_{0} / 2$ and $T_{1}+T_{2}+T_{0} / 2$.

$$
T_{2}+T_{0} / 2=\left(T_{s}-m_{a} \sin (\pi / 6-\theta) T_{s}+m_{a} \sin (\pi / 6+\theta) T_{s}\right) / 2
$$

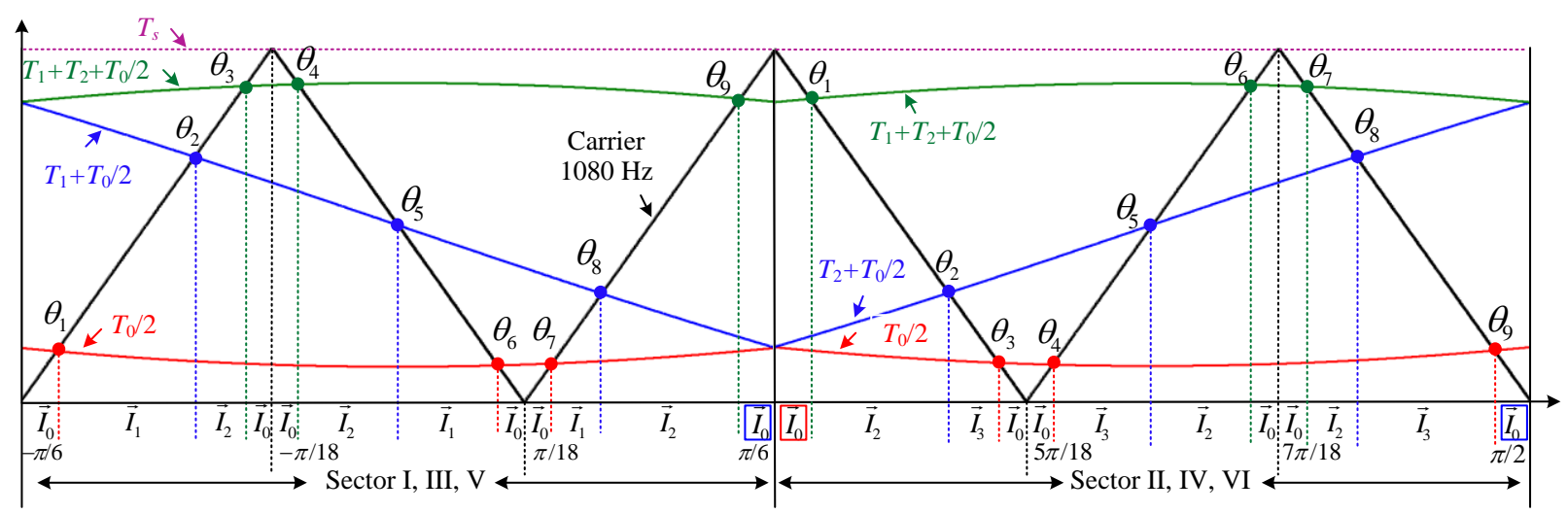

Figure 3-12 Sequence 4. (a) Sector I, III, V. (b) Sector II, IV, VI. 


\section{Case E: Sequence 5}

Space vector sequence 5 (SQ5) shown in Figure 3-8 (e) is generated by removing one zero vector in the center of SQ4. Again, the zero vector (highlighted by a blue block as shown in Figure 3-13) in the last sample in the present sector should be selected as the zero vector for the next sector. To ensure a symmetry waveform, a switching frequency of $480 \mathrm{~Hz}$ together with a sampling frequency of $1440 \mathrm{~Hz}$ is needed.

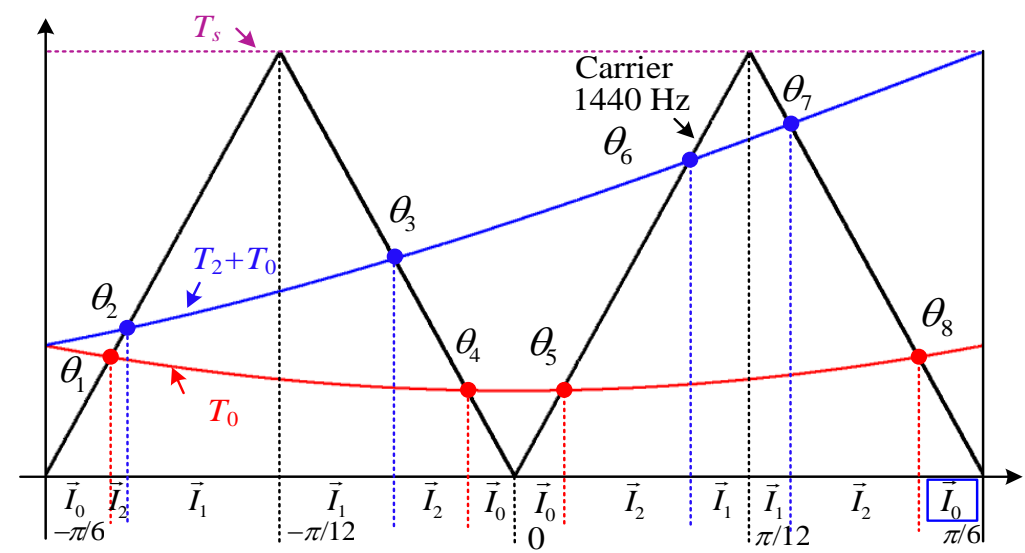

Figure 3-13 Sequence 5 (Sector I).

Similarly, equations of the carriers and the dwell time references $T_{0}$, and $T_{2}+T_{0}$ are expressed as (3-19), (3-3) and (3-20), respectively.

$$
\begin{aligned}
f(\theta)= & (12 \theta / \pi+2) T_{s} \text { for }-\pi / 6 \leq \theta \leq-\pi / 12 \\
f(\theta)= & (-12 \theta / \pi) T_{s} \text { for }-\pi / 12 \leq \theta \leq 0 \\
f(\theta)= & (12 \theta / \pi) T_{s} \text { for } 0 \leq \theta \leq \pi / 12 \\
f(\theta)= & (-12 \theta / \pi+2) T_{s} \text { for } \pi / 12 \leq \theta \leq \pi / 6 \\
& T_{2}+T_{0}=T_{s}-m_{a} \sin (\pi / 6-\theta) T_{s}
\end{aligned}
$$

Case F: Sequence 6

Space vector sequence 6 (SQ6) shown in Figure 3-8 (f) is obtained by removing the zero vectors at both sides of SQ4. As a discontinuous sequence, SQ6 is not symmetrical from the sequence center with advantage of reducing one switching during the sector crossing, where $\overrightarrow{\boldsymbol{I}}_{n+1}$ (highlighted by a blue block as shown in Figure 3-14) in the present sector will be the same as $\overrightarrow{\boldsymbol{I}}_{n}$ in the next sector. A switching/sampling frequency of 540/1440 Hz are required for waveform symmetry. 
SQ6 has eight intersections (dwell times) $\theta_{1}, \theta_{2}, \theta_{3}, \theta_{4}, \theta_{5}, \theta_{6}, \theta_{7}, \theta_{8}$ per sector shown in Figure 3-14. SQ6 is a discontinuous sequence that the eight intersections are calculated by different dwell time references. The carriers are same as those in SQ5, while the dwell time references for $\theta_{1}, \theta_{2}, \theta_{5}, \theta_{6}$ are $T_{1}$ and $T_{1}+T_{2}$, and the dwell time references for $\theta_{3}, \theta_{4}, \theta_{7}, \theta_{8}$ are $T_{2}$ and $T_{1}+T_{2}$. In summary, dwell time equations for different space vector sequences are listed in Table 3-2.
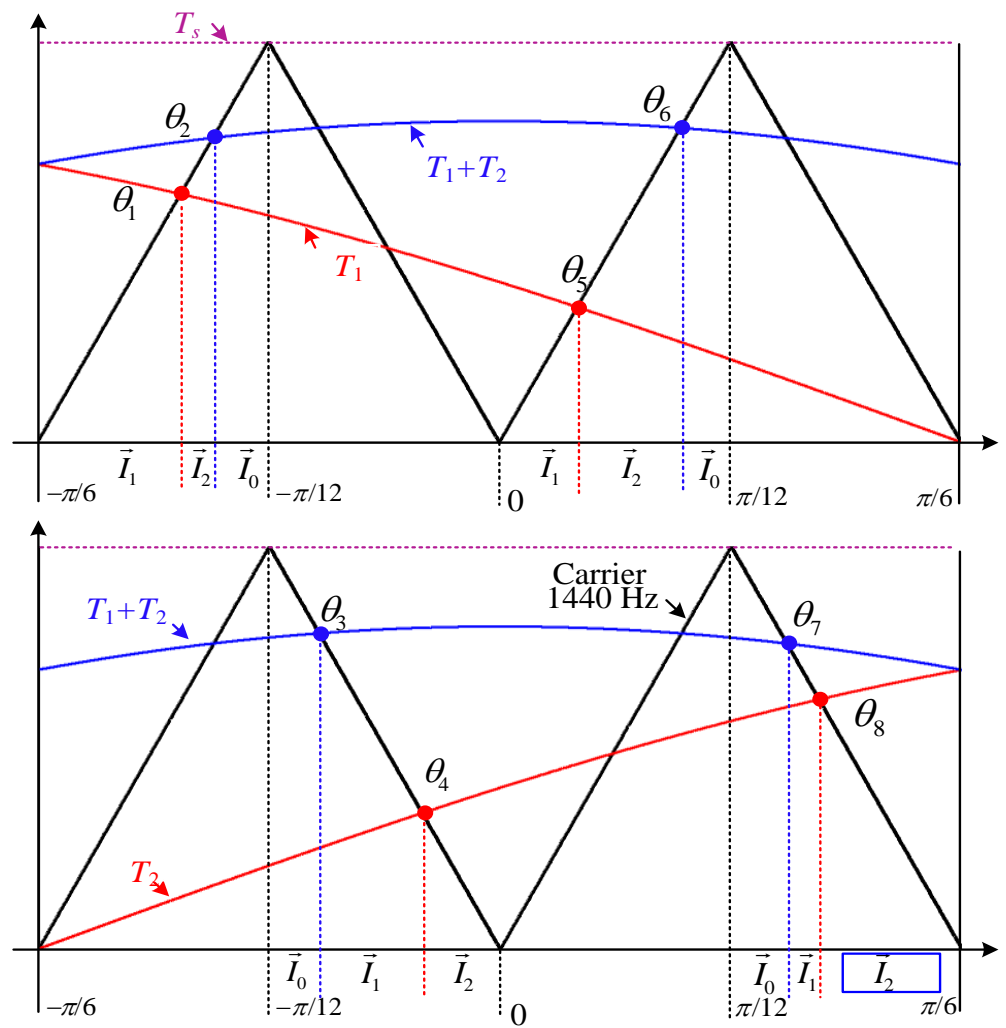

Figure 3-14 Sequence 6 (Sector I).

Table 3-2 Summary of Different Space Vector Sequences

\begin{tabular}{|c|c|c|c|c|}
\hline $\begin{array}{c}\text { Vector } \\
\text { Sequences }\end{array}$ & $\begin{array}{c}\text { Switching } \\
\text { Frequency }\left(f_{s w}\right)\end{array}$ & $\begin{array}{c}\text { Sampling } \\
\text { Frequency }\left(f_{s p}\right)\end{array}$ & $\begin{array}{c}\text { Effective Switching Angles } \\
\theta\end{array}$ & $\begin{array}{c}\text { Derived Equations } \\
\text { for Angles Solving }\end{array}$ \\
\hline SQ1 & $540 \mathrm{~Hz}$ & $1080 \mathrm{~Hz}$ & $\theta_{1}, \theta_{2}, \theta_{3}, \theta_{4}, \theta_{5}, \theta_{6}$ & $(3-1),(3-6),(3-11)$ \\
\hline SQ2 & $540 \mathrm{~Hz}$ & $1080 \mathrm{~Hz}$ & $\theta_{1}, \theta_{2}, \theta_{3}, \theta_{4}, \theta_{5}, \theta_{6}, \theta_{7}, \theta_{8}, \theta_{9}$ & $(3-6),(3-12),(3-13),(3-14)$ \\
\hline SQ3 & $480 \mathrm{~Hz}$ & $1080 \mathrm{~Hz}$ & $\theta_{1}, \theta_{2}, \theta_{3}, \theta_{4}, \theta_{5}, \theta_{6}$ & $(3-1),(3-6),(3-15)$ \\
\hline \multirow{2}{*}{ SQ4 } & $540 \mathrm{~Hz}$ & $1080 \mathrm{~Hz}$ & $\begin{array}{c}\theta_{1}, \theta_{2}, \theta_{3}, \theta_{4}, \theta_{5}, \theta_{6}, \theta_{7}, \theta_{8}, \theta_{9} \\
\text { Sector I, III, V }\end{array}$ & $(3-12),(3-13),(3-14),(3-16)$ \\
\cline { 4 - 5 } & & & $\begin{array}{c}\theta_{1}, \theta_{2}, \theta_{3}, \theta_{4}, \theta_{5}, \theta_{6}, \theta_{7}, \theta_{8}, \theta_{9} \\
\text { Sector II, IV, VI }\end{array}$ & $(3-12),(3-14),(3-17),(3-18)$ \\
\hline SQ5 & $480 \mathrm{~Hz}$ & $1440 \mathrm{~Hz}$ & $\theta_{1}, \theta_{2}, \theta_{3}, \theta_{4}, \theta_{5}, \theta_{6}, \theta_{7}, \theta_{8}$ & $(3-3),(3-19),(3-20)$ \\
\hline \multirow{2}{*}{$\mathrm{SQ6}$} & $540 \mathrm{~Hz}$ & $1440 \mathrm{~Hz}$ & $\theta_{1}, \theta_{2}, \theta_{5}, \theta_{6}$ & $(3-1),(3-11),(3-19)$ \\
\cline { 4 - 5 } & & & $\theta_{3}, \theta_{4}, \theta_{7}, \theta_{8}$ & $(3-2),(3-11),(3-19)$ \\
\hline
\end{tabular}




\subsubsection{Comparison among different sequences}

Figure 3-15 shows the low-order harmonics performance of the six sequences based on NSSVM. SQ1 and SQ2 perform better low-order harmonics in the full operation range compared with other sequences. Even though the magnitude of the 7th harmonic of SQ6 around $m_{a}=0.6$ is the lowest one among the six sequences, SQ6 cannot be used in practice as the 5th harmonic in the full operation range and the 7th in high and low modulation index are very high. Compared with SQ1, SQ2 offers better performance in the full operation range.

Therefore, SQ2 is the best one in terms of low-order harmonics performance (5th and 7th) among all the six space vector sequences in the full operation range.
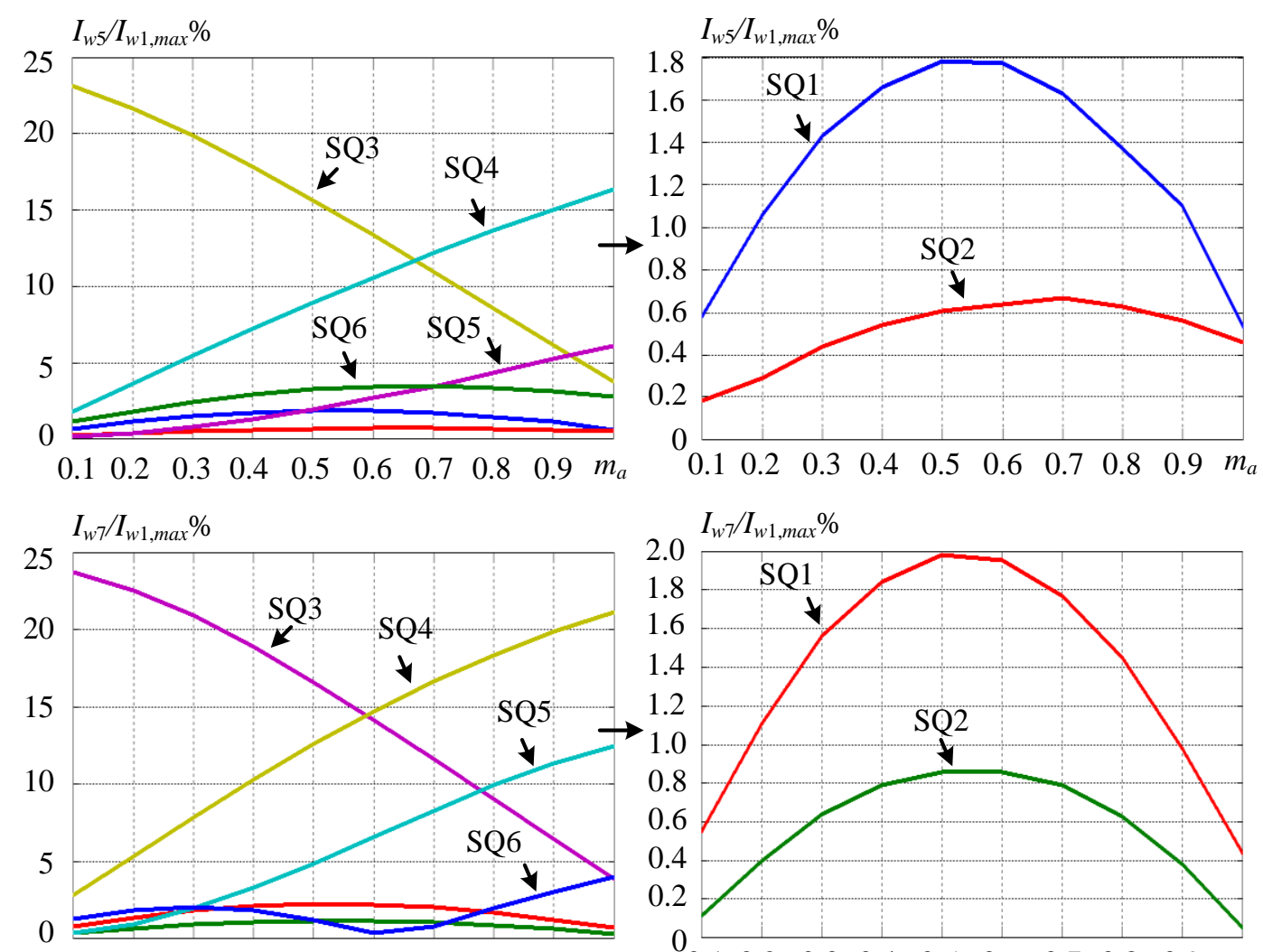

$\begin{array}{llllllllll}0.1 & 0.2 & 0.3 & 0.4 & 0.5 & 0.6 & 0.7 & 0.8 & 0.9 & m_{a}\end{array}$

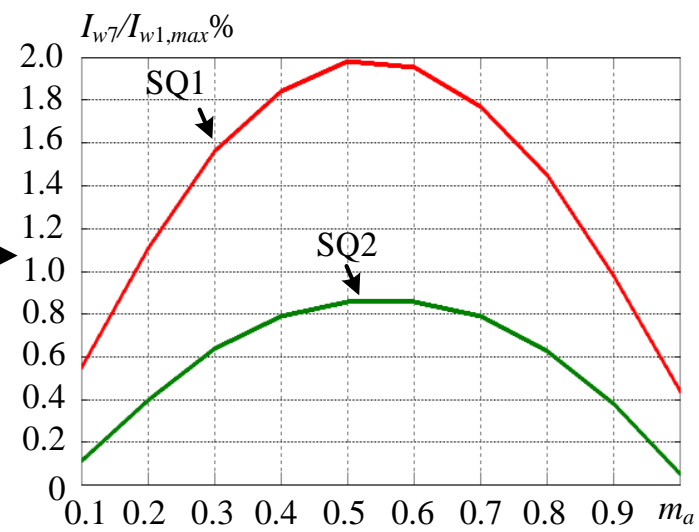

Figure 3-15 Low-order harmonics performance among different sequences.

One main step of the NS-SVM is to calculate the natural sampling based dwell times online. As mentioned earlier, they can be obtained by the two-step equations derived based on NewtonRaphson algorithm. However, they are complex and will increase computational burden. To solve this problem, one-step equations are introduced. The one-step equations contribute to lower 
computational burden, but with similar low-order harmonics performance as illustrated in Figure 3-16. The final simplified one-step equations are shown in Table 3-3.
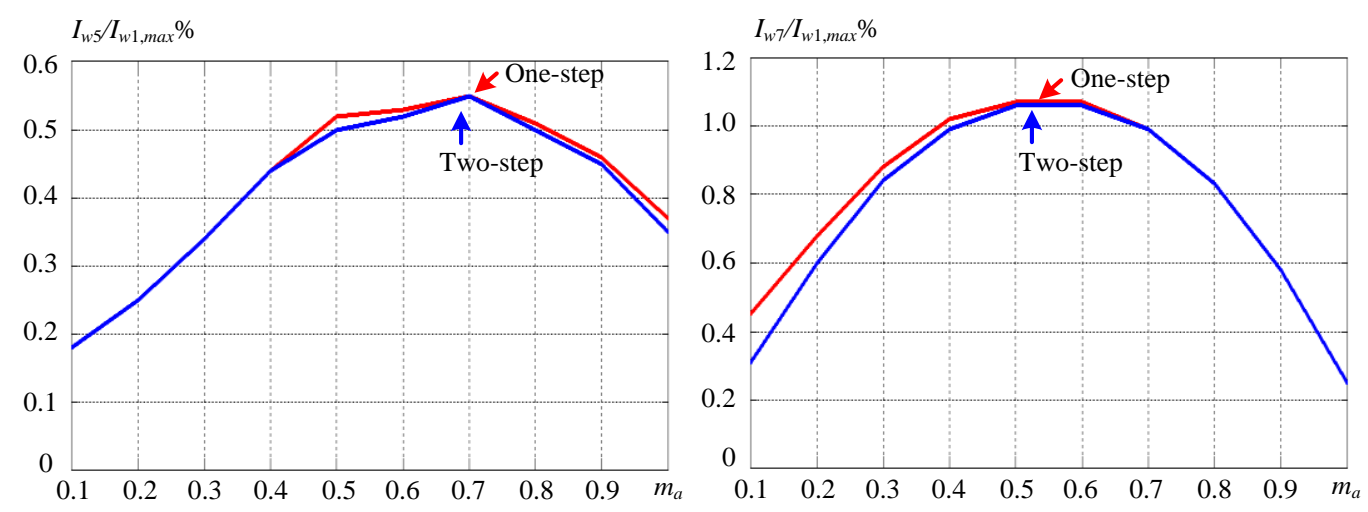

Figure 3-16 Comparison of low-order harmonics between one-step and two-step equations.

Table 3-3 Online Dwell Times Equations for SQ2

\begin{tabular}{|l|l|}
\hline$\theta_{1}$ & $-\pi / 6+\frac{1 / 2\left(m_{a} \sin \left(\pi / 6-\theta_{10}\right)+m_{a} \sin \left(\pi / 6+\theta_{10}\right)\right)+\left(9 \theta_{10} / \pi+1\right)}{1 / 2\left(m_{a} \cos \left(\pi / 6-\theta_{10}\right)-m_{a} \sin \left(\pi / 6+\theta_{10}\right)\right)-9 / \pi}$ \\
\hline$\theta_{2}$ & $\theta_{1}+\frac{1 / 2\left(-m_{a} \sin \left(\pi / 6-\theta_{20}\right)+m_{a} \sin \left(\pi / 6+\theta_{20}\right)\right)+\left(9 \theta_{20} / \pi+1\right)}{1 / 2\left(-m_{a} \cos \left(\pi / 6-\theta_{20}\right)-m_{a} \cos \left(\pi / 6+\theta_{20}\right)\right)-9 / \pi}$ \\
\hline$\theta_{3}$ & $\theta_{2}+\frac{1 / 2\left(-m_{a} \sin \left(\pi / 6-\theta_{30}\right)-m_{a} \sin \left(\pi / 6+\theta_{30}\right)\right)+\left(9 \theta_{30} / \pi+1\right)}{1 / 2\left(-m_{a} \cos \left(\pi / 6-\theta_{30}\right)+m_{a} \cos \left(\pi / 6+\theta_{30}\right)\right)-9 / \pi}$ \\
\hline$\theta_{4}$ & $-\pi / 18+\frac{1 / 2\left(m_{a} \sin \left(\pi / 6-\theta_{40}\right)+m_{a} \sin \left(\pi / 6+\theta_{40}\right)\right)+9 \theta_{40} / \pi}{1 / 2\left(m_{a} \cos \left(\pi / 6-\theta_{40}\right)-m_{a} \sin \left(\pi / 6+\theta_{40}\right)\right)-9 / \pi}$ \\
\hline$\theta_{5}$ & $\theta_{4}+\frac{1 / 2\left(-m_{a} \sin \left(\pi / 6-\theta_{50}\right)+m_{a} \sin \left(\pi / 6+\theta_{50}\right)\right)+9 \theta_{50} / \pi}{1 / 2\left(-m_{a} \cos \left(\pi / 6-\theta_{50}\right)-m_{a} \cos \left(\pi / 6+\theta_{50}\right)\right)-9 / \pi}$ \\
\hline$\theta_{6}$ & $\theta_{5}+\frac{1 / 2\left(-m_{a} \sin \left(\pi / 6-\theta_{60}\right)-m_{a} \sin \left(\pi / 6+\theta_{60}\right)\right)+9 \theta_{60} / \pi}{1 / 2\left(-m_{a} \cos \left(\pi / 6-\theta_{60}\right)+m_{a} \cos \left(\pi / 6+\theta_{60}\right)\right)-9 / \pi}$ \\
\hline$\theta_{7}$ & $\pi / 18+\frac{1 / 2\left(m_{a} \sin \left(\pi / 6-\theta_{70}\right)+m_{a} \sin \left(\pi / 6+\theta_{70}\right)\right)+\left(9 \theta_{40} / \pi-1\right)}{1 / 2\left(m_{a} \cos \left(\pi / 6-\theta_{70}\right)-m_{a} \sin \left(\pi / 6+\theta_{70}\right)\right)-9 / \pi}$ \\
\hline$\theta_{8}$ & $\theta_{7}+\frac{1 / 2\left(-m_{a} \sin \left(\pi / 6-\theta_{80}\right)+m_{a} \sin \left(\pi / 6+\theta_{80}\right)\right)+\left(9 \theta_{80} / \pi-1\right)}{1 / 2\left(-m_{a} \cos \left(\pi / 6-\theta_{80}\right)-m_{a} \cos \left(\pi / 6+\theta_{80}\right)\right)-9 / \pi}$ \\
\hline$\theta_{9}$ & $\theta_{8}+\frac{1 / 2\left(-m_{a} \sin \left(\pi / 6-\theta_{90}\right)-m_{a} \sin \left(\pi / 6+\theta_{90}\right)\right)+\left(9 \theta_{90} / \pi-1\right)}{1 / 2\left(-m_{a} \cos \left(\pi / 6-\theta_{90}\right)+m_{a} \cos \left(\pi / 6+\theta_{90}\right)\right)-9 / \pi}$ \\
\hline
\end{tabular}




\subsection{Experimental verification}

\subsubsection{Comparison between conventional SVM and NS-SVM}

Figure 3-17 and Figure 3-18 illustrate the experimental waveforms of the converter output PWM current $i_{w}$, line current $i_{s}$ and their FFT analysis under conditions of $f_{s w}=540 \mathrm{~Hz}$ and $m_{a}=$ 1. In the case of conventional SVM shown in Figure 3-17, the $5^{\text {th }}$ and $7^{\text {th }}$ harmonics are approximately $11 \%$ and $7.0 \%$, respectively. The $11^{\text {th }}$ and $13^{\text {th }}$ are around $7.3 \%$ and $7.2 \%$. The low-order harmonics performance of NS-SVM, on the other hand, is superior as shown in Figure $3-18$, that are $0.6 \%$ (5th), $0.7 \%$ (7th), $1.1 \%$ (11th), and $1.66 \%$ (13th), respectively.
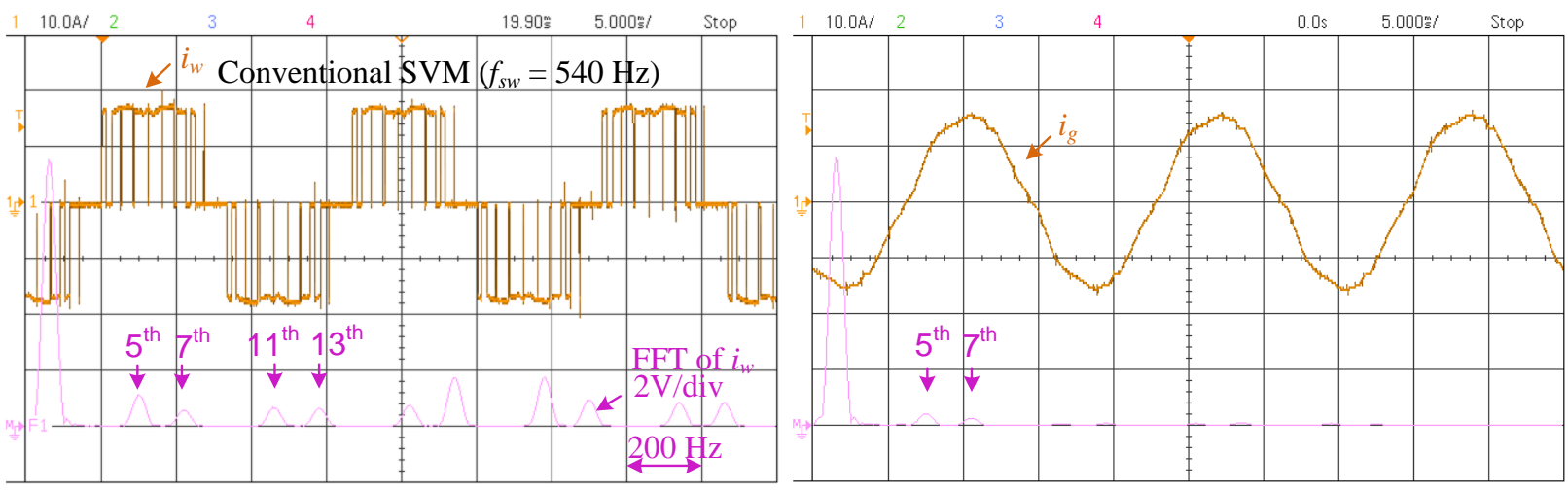

Figure 3-17 Experimental waveforms based on conventional SVM.
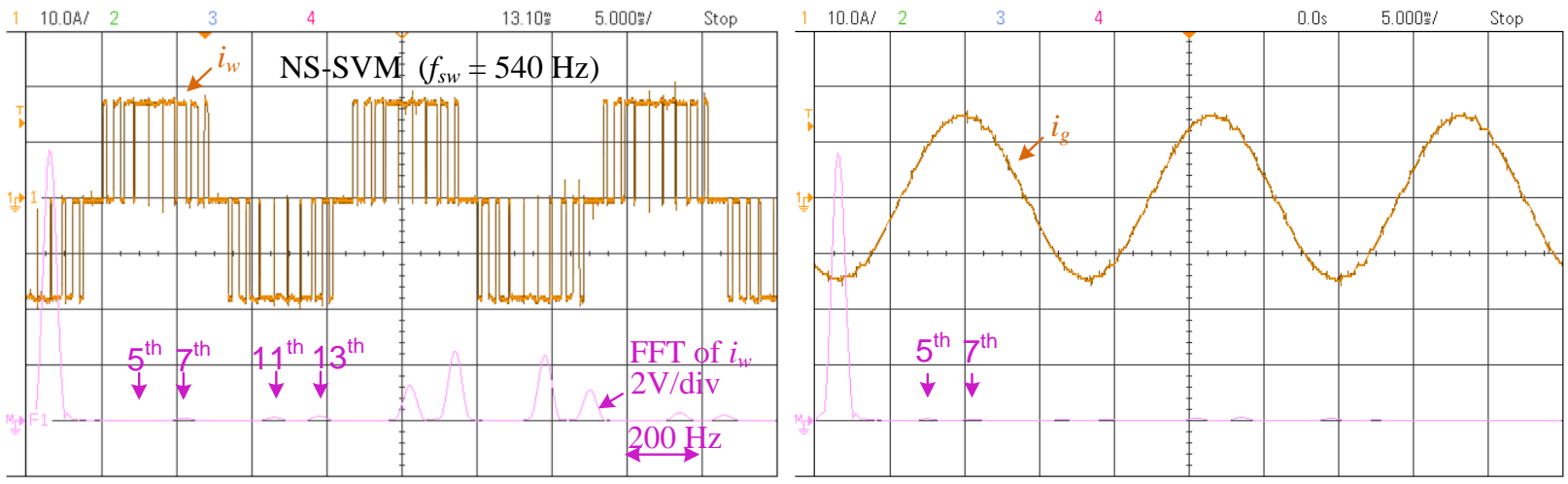

Figure 3-18 Experimental waveforms based on NS-SVM.

In summary, NS-SVM performs better low-order harmonics than conventional SVM. Though the magnitudes of the high-order harmonics (17th, 19th, 23th and 25th) of NS-SVM are higher than conventional SVM, they are effectively damped by the LC filter shown in Figure 3-18. 


\subsubsection{Comparison among different sequences}

Figure 3-19 shows experimental waveforms of the converter output PWM current $i_{w}$ and their FFT analysis of different vector sequences. All the experiments are conducted based on $m_{a}=1$. The resultant switching frequencies for different sequences may be different as previously mentioned. SQ3 and SQ5 are with $480 \mathrm{~Hz}$, while SQ1, SQ2, SQ4, and SQ6 are with $540 \mathrm{~Hz}$.

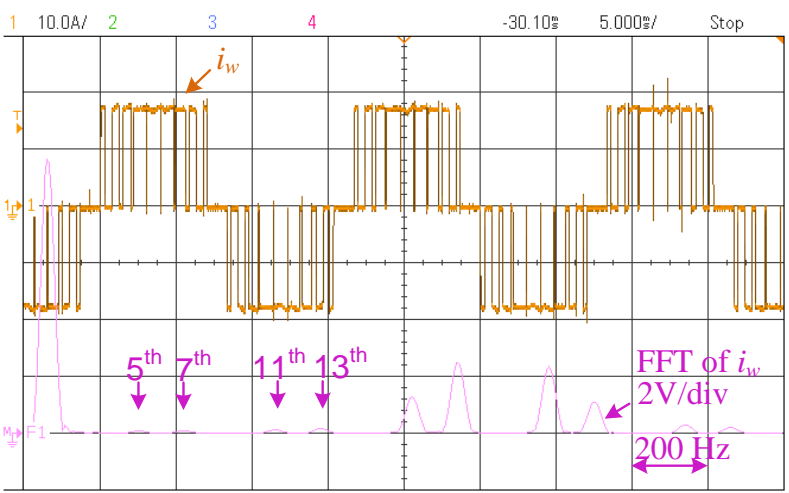

(a) SQ1

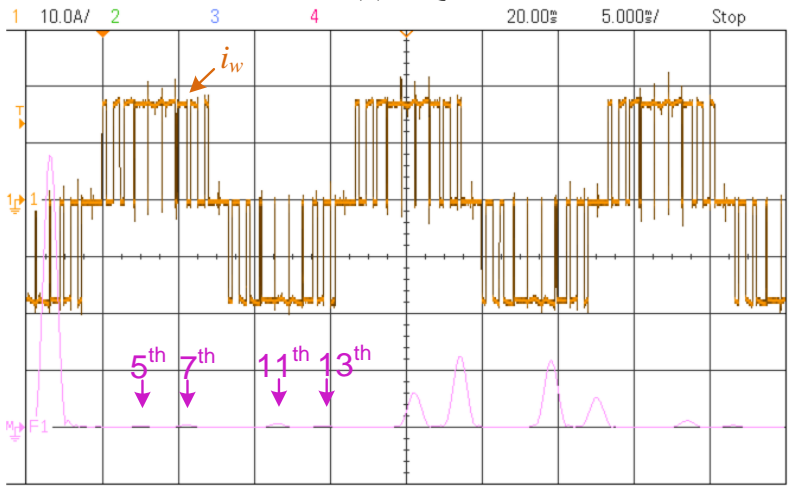

(b) SQ2

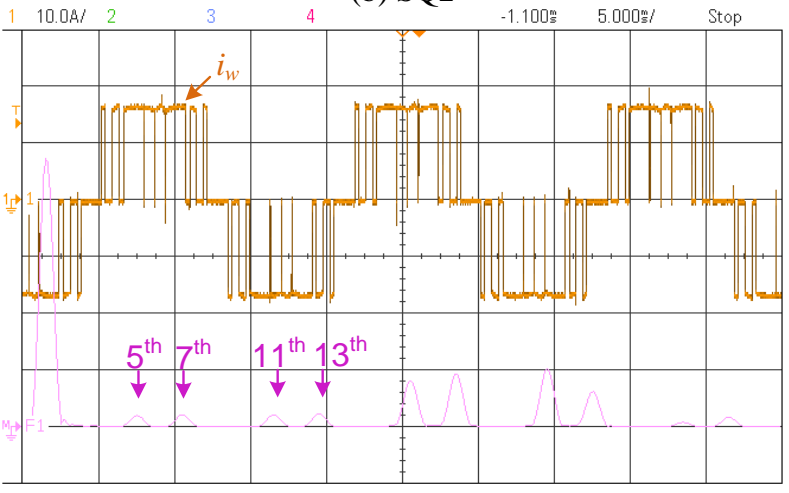

(c) SQ3

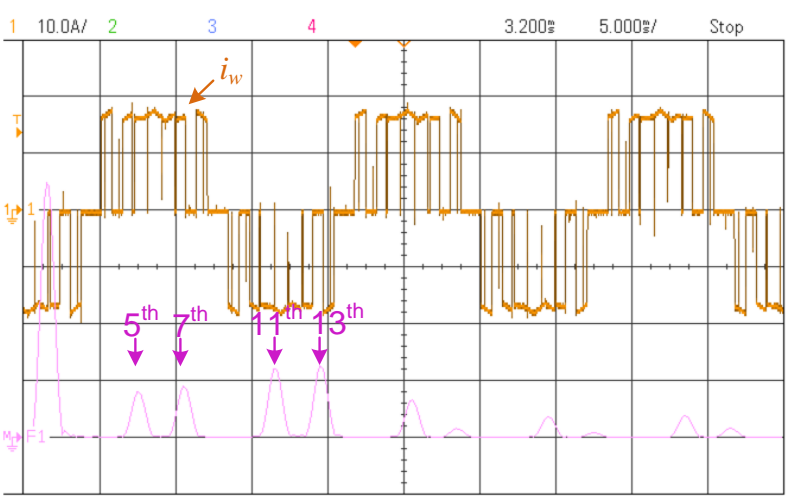

(d) SQ4

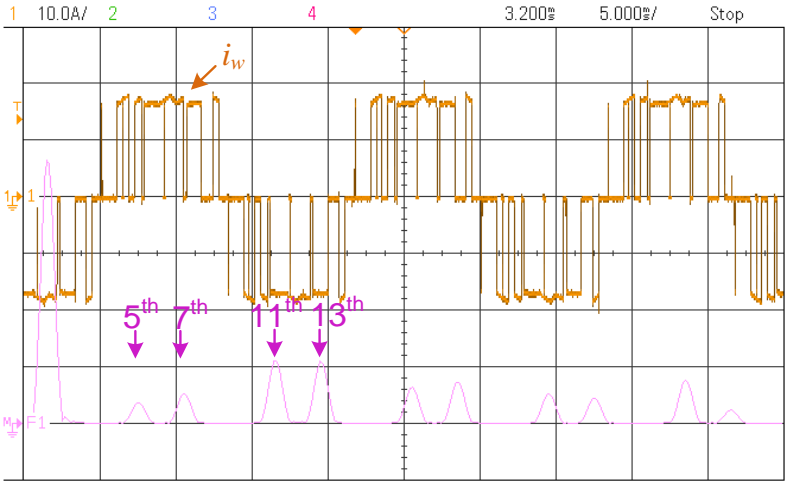

(e) SQ5

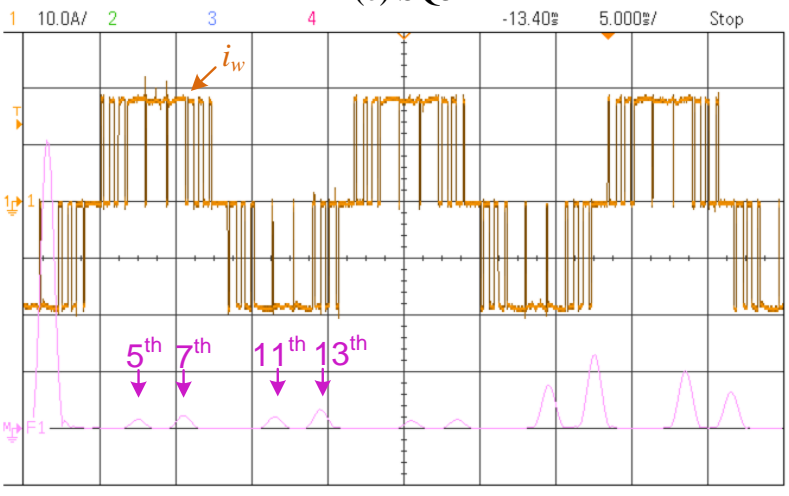

(f) SQ6

Figure 3-19 Experimental waveforms of different sequences under unity modulation index.

Obviously, SQ1 and SQ2 perform better in terms of low-order harmonics (5th and 7th) compared with other sequences. 
Figure 3-20 and Figure 3-21 show the experimental waveforms of converter output PWM current $i_{w}$ and line current $i_{s}$ of SQ1 and SQ2 under $f_{s w}=540 \mathrm{~Hz}, f_{L C}=4.5 \mathrm{pu}, m_{a}=0.5, R_{g}=$ $0.01 \mathrm{pu}$. SQ2 performs better low-order harmonics performance than SQ1.

In summary, the four-segment sequence (SQ2) based on NS-SVM is the best one in terms of low-order harmonics performance among the six investigated sequences.
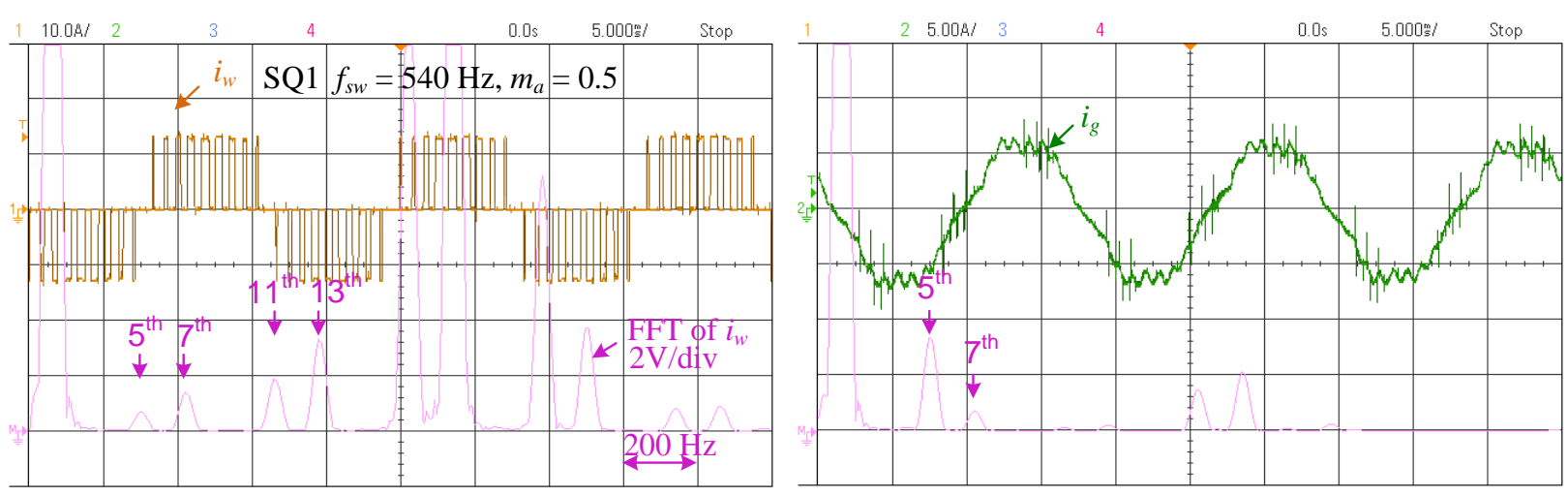

Figure 3-20 Experimental waveforms of SQ1.
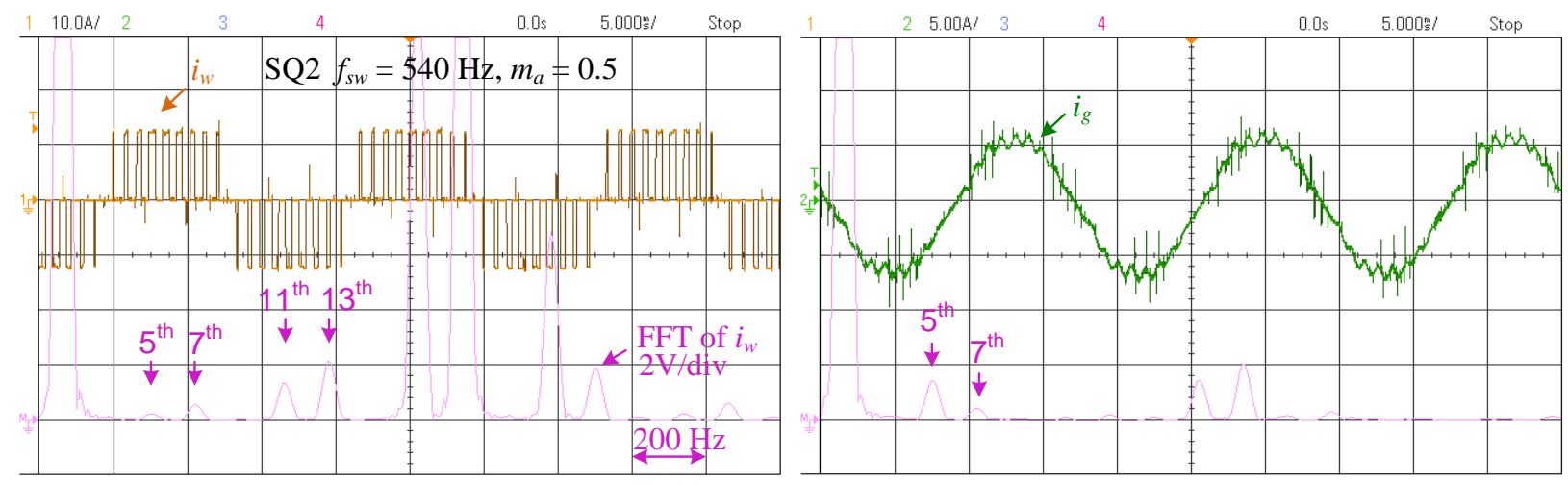

Figure 3-21 Experimental waveforms of SQ2.

\subsection{Conclusions}

In commercial grid-side MV CSCs, only the selective harmonic elimination (SHE) is used. SHE features superior low-order harmonics performance but with low dynamic response as it is an off-line scheme. SVM featuring fast dynamic response, however, cannot be used for the gridside MV CSC mainly due to its high-magnitude low-order harmonics. A multi-sampling SVM can improve the performance of the low-order harmonics, but with the penalty of higher switching/sampling frequency which is not preferred for high-power MV applications. 
In this chapter, a natural sampling SVM (NS-SVM) with superior low-order harmonics performance, is proposed for grid-side MV CSCs. Compared with conventional SVM, NS-SVM features superior low-order harmonics performance in the full operation range without compromising performance in terms of fast dynamic performance and low switching/sampling frequency. Compared with the multi-sampling SVM, NS-SVM does not introduce additional switching frequency, but with superior low-order harmonics performance that is equal to the performance when the sampling frequency of the multi-sampling SVM increases to infinite.

Besides, the optimal space vector sequence among existing main sequences for CSCs in terms of low-order harmonics performance is investigated. Among these investigated sequences, the four-segment sequence based on NS-SVM performs best low-order harmonics performance in the full operation range.

Experiments are carried out to verify the performance of NS-SVM and the selected optimal space vector sequence. 


\section{Chapter 4}

\section{Power Balancing Control of Series-}

\section{Connected CSCs}

In the proposed CSC-based offshore wind farm configuration, a number of MV CSCs are connected in series to form a centralized dc/ac inverter transferring the wind power to the grid. An equal power distribution among all the CSCs should be ensured during operation. Otherwise, the system reliability may suffer. For example, the one that contributes a greater portion of the power will be thermally overstressed. In previous studies, the series-connected CSCs are controlled based on assumptions that all CSCs are ideal and identical and the power is evenly distributed among them. However, in practice, such assumptions are invalid as tolerance of components exists. Therefore, in this chapter, the investigation of power distribution among series-connected CSCs considering the tolerance is conducted and a power balancing control scheme to ensure an even distribution of power among CSCs is proposed.

This chapter starts with a brief introduction of conventional control scheme of the seriesconnected CSCs followed by the proposed one. First, the possible power imbalance is discussed and concluded. Second, the possible imbalance is quantitatively defined. Third, a power balancing scheme is proposed that an even distribution of power among CSCs is ensured. Both simulation and experimental results are provided to verify the effectiveness of the scheme.

\subsection{Control objectives of series-connected CSCs}

Figure 4-1 shows the series-connected CSCs in the proposed system. Figure 4-2 shows the conventional control scheme of the series-connected CSCs. There are two control objectives for the series-connected CSCs when used in offshore wind farms. One is the dc-link current control and the other is the power factor control. The dc-link reference current $I_{d c_{-} r e f}$ and the reactive power $Q_{r e f}$ are received from the wind farm supervisory control and grid codes, respectively [40]. The outputs of the dc-link current and reactive power controllers are $d$-axis grid current reference $\left(I_{g d \_r e f}\right)$ and $q$-axis grid current reference $\left(I_{g q_{-} r e f}\right)$, respectively. The converter reference current 
( $\left.I_{w d \_r e f}, I_{w q \_} r e\right)$ is obtained by compensating the grid-side filter capacitor current. The modulation index $m_{a}$ and delay angle $\alpha$ are calculated based on the grid converter reference current and gating signals are generated using SVM and then applied to all the CSCs. Please refer to [40] for more details.

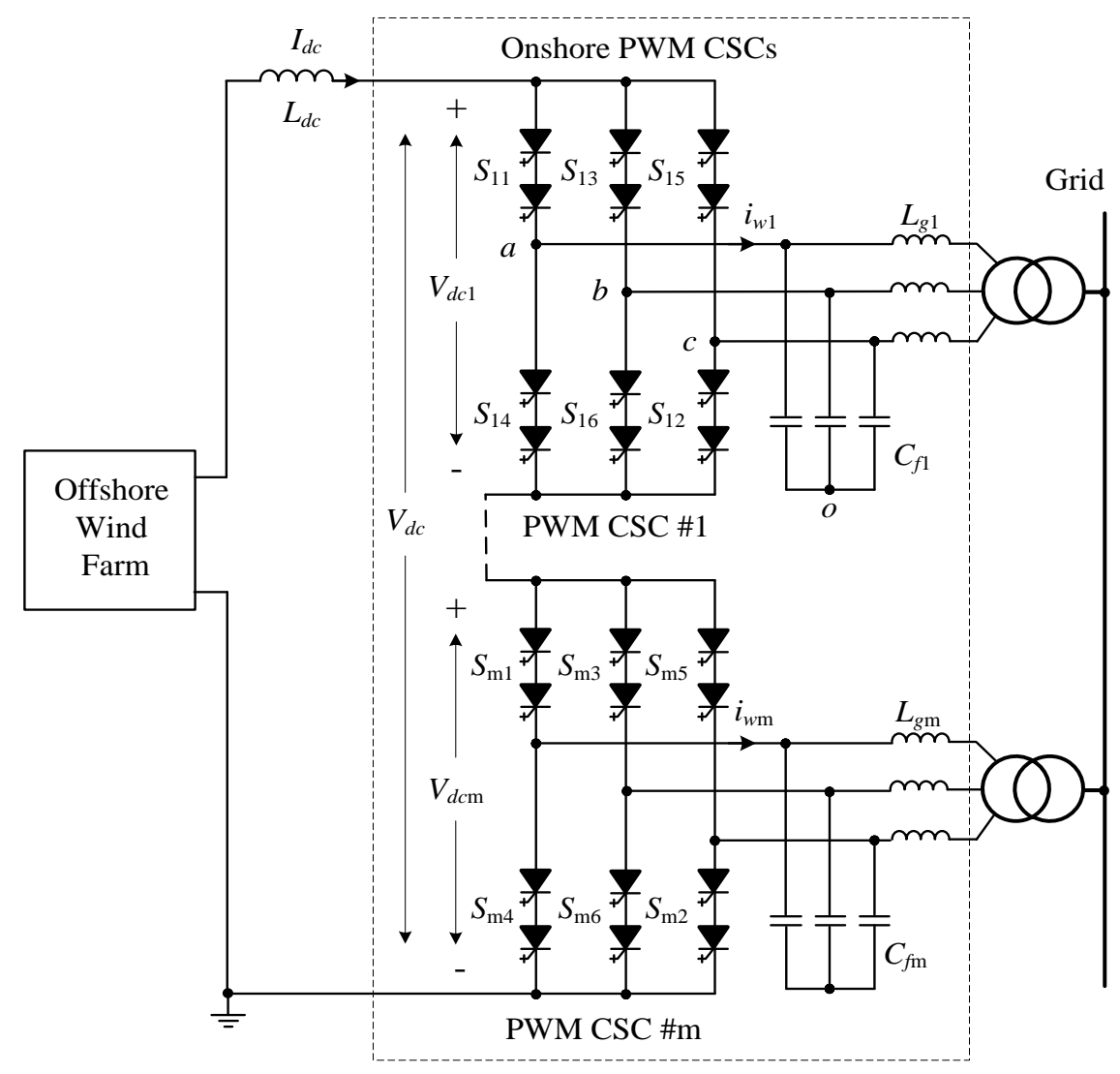

Figure 4-1 Series-connected MV CSCs.

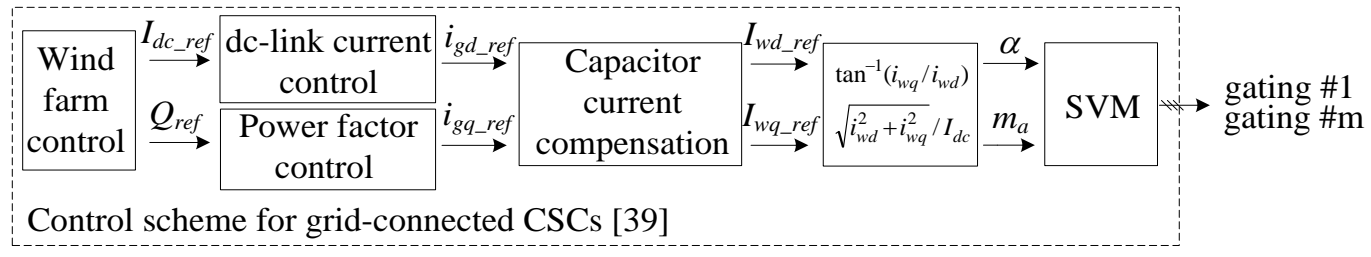

Figure 4-2 Conventional control scheme for series-connected CSCs [40].

Conventional scheme assumes that all the CSCs and passive components are ideal and identical that equal power distribution can be achieved by applying identical switching signal ( $m_{a}$ and $\alpha$ ) to all the CSCs [40]. However, it is not the case at all in practice where tolerance exists. The power balance among CSCs must be ensured during operation. Otherwise, the system reliability suffers. For example, the one with a higher power will be thermally overstressed. In summary, 
the question goes to if the tolerance of components in practice will introduce power imbalance and how much. This will be answered in the following [69].

\subsection{Power and input voltage of CSCs}

\subsubsection{Relationship between power and input voltage}

As shown in Figure 4-1, the CSCs are connected in series and share one dc-link current, that is $I_{d c}$. The dc-link voltage of the system, that is $V_{d c}$ is equal to the sum of all the input voltage of each CSC, that is $V_{d c \mathrm{~m}}$. Based on power conservation, the power of each CSC will be automatically balanced once its input voltage is balanced. For simplicity, the balance of input voltage of the CSCs will be discussed in the following.

\subsubsection{Instantaneous input voltage}

The instantaneous input voltage of each CSC $v_{d c m}$ is clamped by the grid voltage. Taking Module \#1 as an example. Assuming at one instant, $S_{11}$ and $S_{16}$ are on, the input voltage $v_{d c 1}$ is equal to $v_{a o}-v_{b o}$ (the on-state voltages of the switching devices are too small that can be neglected) as shown in Figure 4-1. Similarly, $v_{d c}$ under other switching states can be obtained with the same manner and they are illustrated in Table 4-1. Figure 4-3 shows the instantaneous input voltage $v_{d c 1}$ of Module \#1 during one fundamental period where the modulation index $m_{a}$ and the delay angle $\alpha$ are set to 0.8 and 0 , respectively.

As the capacitor voltage is clamped by the grid voltage, the instantaneous input voltage of each CSC is therefore clamped by the grid and the instantaneous input voltage of each CSC will be well balanced.

Table 4-1 Switching States and Input Voltage

\begin{tabular}{|c|c|c|c|}
\hline Type & $\begin{array}{c}\text { Switching } \\
\text { States }\end{array}$ & $\begin{array}{c}\text { On-State } \\
\text { Switch }\end{array}$ & $\begin{array}{c}\text { Input } \\
\text { Voltage } v_{d}\end{array}$ \\
\hline \multirow{4}{*}{$\begin{array}{c}\text { Zero } \\
\text { States }\end{array}$} & {$[14]$} & $S_{11}, S_{14}$ & \multirow{2}{*}{0} \\
\cline { 2 - 3 } & {$[36]$} & $S_{13}, S_{16}$ & 0 \\
\cline { 2 - 3 } & {$[52]$} & $S_{15}, S_{12}$ & \\
\hline \multirow{5}{*}{ Active } & {$[61]$} & $S_{16}, S_{11}$ & $v_{a o}-v_{b o}$ \\
\cline { 2 - 4 } States & {$[12]$} & $S_{11}, S_{12}$ & $v_{a o}-v_{c o}$ \\
\cline { 2 - 4 } & {$[23]$} & $S_{12}, S_{13}$ & $v_{b o}-v_{c o}$ \\
\cline { 2 - 4 } & {$[34]$} & $S_{13}, S_{14}$ & $v_{b o}-v_{a o}$ \\
\cline { 2 - 4 } & {$[45]$} & $S_{14}, S_{15}$ & $v_{c o}-v_{a o}$ \\
\cline { 2 - 4 } & {$[56]$} & $S_{15}, S_{16}$ & $v_{c o}-v_{b o}$ \\
\hline
\end{tabular}




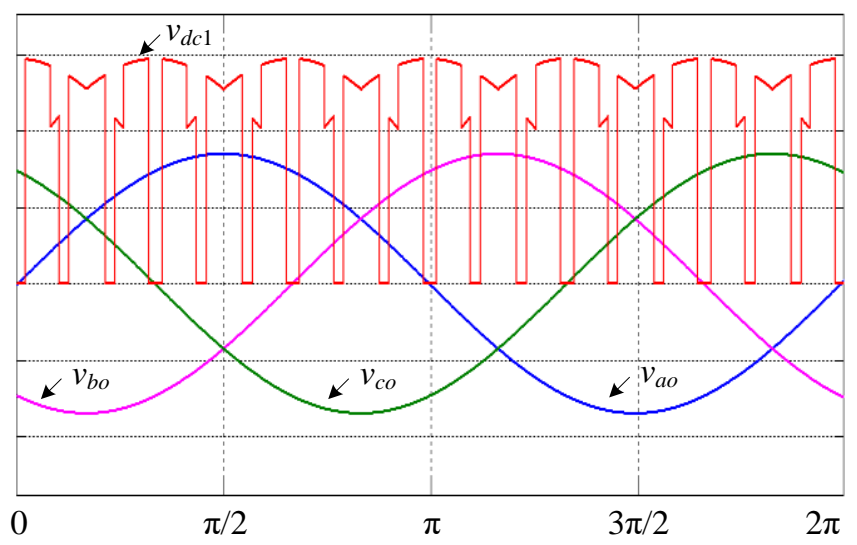

Figure 4-3 Input voltage during one fundamental period $\left(\mathrm{m}_{\mathrm{a}}=0.8, \alpha=0\right)$.

\subsubsection{Average input voltage}

The average input voltage of each CSC is derived based on the rule of energy conservation. The input power is given by

$$
P_{i n}=V_{d c} I_{d c}
$$

where $V_{d c}$ and $I_{d c}$ are the average dc voltage and dc-link current, respectively.

The output power for each CSC can be expressed as

$$
P_{\text {out }}=\sqrt{3} V_{L L} I_{w 1} \cos (\alpha)
$$

where $V_{L L}$ is the rms line-to-line voltage of the grid, $I_{w 1}$ is the rms value of the fundamentalfrequency current of $i_{w}, \alpha$ is the phase displacement between the grid voltage and the fundamental current of the CSC output PWM current $i_{w}$.

Neglecting the power losses in the inverter, the input power $P_{i n}$ is equal to the output power $P_{\text {out }}$,

$$
\sqrt{3} V_{L L} I_{w 1} \cos (\alpha)=V_{d c} I_{d c}
$$

from which

$$
V_{d c}=\sqrt{3 / 2} V_{L L} m_{a} \cos (\alpha)
$$

where $m_{a}=\sqrt{2} I_{w 1} / I_{d c}$. 
In summary, the peak value of the instantaneous input voltage of each CSC clamped by the grid voltage will be well balanced, while the average input voltage which is related to both the modulation index and the delay angle shown in (4-4) may not be balanced during operation. This will be investigated in the next section.

\subsection{Input voltage distribution among CSCs}

This section investigates the power distribution among CSCs. The possible imbalance of power among CSCs is unveiled and quantitatively defined. As mentioned earlier, the power will be automatically balanced when the average input voltage is balanced. And the latter will be discussed in the following.

\subsubsection{Voltage distribution under practical conditions}

In practice, the tolerances include the possible unequal on-state voltages of switching devices, the possible mismatch of gating signals, and the tolerance in filter parameters.

(a) Unequal on-state voltage of switches

The effect of unequal on-state voltage of switches on the average input voltage distribution are very limited that can be neglected. Taking a $1 \mathrm{MW}, 4160 \mathrm{~V}$ CSC as an example where the rated dc-link current is around 200 A. The commercial switches for MV CSC are SGCT [70] with the maximum on-state voltage being around $5.78 \mathrm{~V}$. Even an impractically huge tolerance, 100\%, for example, can only introduce a limited imbalance, that is $200 * 5.78 \mathrm{~W}$ which is about $0.1 \%$ of its rated power.

(b) Delay in gating signals

A mismatch (delay) in each gating signal possibly occurs in practice. However, it affects neither the peak value of the instantaneous input voltage nor its average input voltage. First, the instantaneous input voltage, a chopped one, is clamped by the grid voltage as shown in Table 4-1. Second, the average input voltage is not affected by the delay in gating signals which can be verified by (4-4).

(c) Tolerance in filter parameters

As mentioned earlier, the control objectives achieved by series-connected CSCs are dc-link current control and power factor control. Here, in the case of a wind farm, the reactive power 
required by grid codes is normally achieved by adding extra reactive support equipment at the plant level, such as SVC and STATCOM. Thus, UPF is used as an example in the following.

Figure 4-4 illustrates the phasor diagrams of the CSC, where all the voltage and current phasors, such as $I_{g}, I_{w}$, and $V_{c}$, represent their fundamental-frequency components only. The subscript " 1 " designated for the fundamental frequency is omitted for simplicity. Figure 4-4 (a) shows the phasor diagram under ideal condition. The control objective is UPF that the line current $I_{g}$ is in phase with grid voltage $V_{g}$. To achieve this, the capacitor current, that is $I_{C_{-} U P F}$, needs to be compensated. The resultant reference PWM current $I_{w_{-} r e f}$ is then obtained. Figure 4-4 (b) illustrates the phasor diagram under a positive tolerance of $C_{f}(+20 \%)$. The capacitor current required to be compensated to achieve UPF is increased by a factor of approximate 1.2, while the reference PWM current of $I_{w_{-}}$ref calculated based on an ideal condition is remaining the same. The resultant line current $I_{g}$ is lagging the grid voltage. The UPF control is failed. Similarly, a leading power factor is produced when the tolerance of $C_{f}$ is negative as shown in Figure 4-4 (c). The phasor diagram under the condition of inductor tolerance can be obtained with the same manner, thus not repeated here.

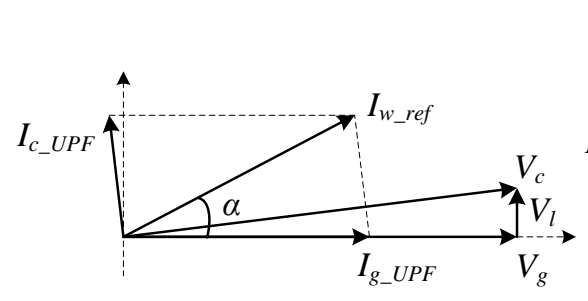

(a) Unity PF

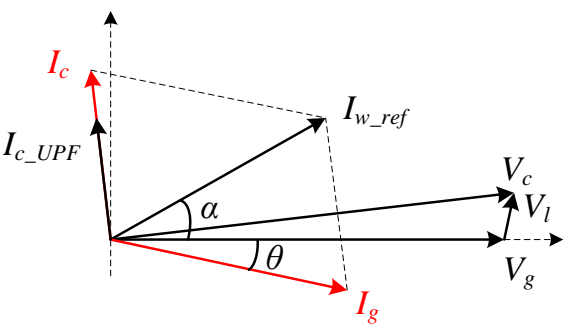

(b) Lagging PF

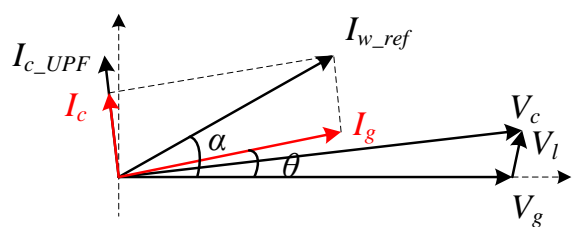

(c) Leading PF

Figure 4-4 Phasor diagram under different conditions.

Based on the above phasor diagram, the capacitor and inductor voltage/current are expressed as follows. 


$$
\begin{aligned}
& I_{w_{-} r e f}=I_{c_{-} U P F}+I_{g_{-} U P F} \\
& I_{g}=I_{w_{-} r e f}-I_{c} \\
& I_{c}=j \omega C_{f} V_{c} k_{1}\left(0.8 \leq k_{1} \leq 1.5\right) \\
& V_{c}=V_{l}+V_{g} \\
& V_{l}=j \omega L_{g} I_{g} k_{2}\left(0.8 \leq k_{2} \leq 1.2\right)
\end{aligned}
$$

where $I_{w_{-} r e f}$ is the common reference PWM current for all CSCs, $I_{C_{-} U P F}$ and $I_{g_{-} U P F}$ are the capacitor and line currents under UPF, $k_{1}$ and $k_{2}$ are tolerance coefficients for the capacitor and inductor in practice, and the others are same as those defined above. Note that the maximum tolerance for such the filter-based capacitors can be ranging from $-20 \%$ to $+20 \%$ or even to $+50 \%$ [71]. The inductance normally has a maximum tolerance within $\pm 20 \%$.

The resultant reference current $I_{w_{-} r e f}$ and line current $I_{g}$ are expressed as

$$
\begin{gathered}
I_{w_{-} r e f}=\left(1-\omega^{2} C_{f} L_{g}\right) I_{g-U P F}+j \omega C_{f} V_{g} \\
I_{g}=\frac{\left(1-\omega^{2} C_{f} L_{g}\right) I_{g-U P F}+j \omega C_{f} V_{g}\left(1-k_{1}\right)}{\left(1-\omega^{2} C_{f} L_{g} k_{1} k_{2}\right)}
\end{gathered}
$$

Accordingly, the resultant grid-side power factor is illustrated in Figure 4-5. First, the tolerance in capacitance leads to the tolerance in power factor. For example, a lagging power factor of 0.87 is received under conditions of $I_{g_{-} U P F}=0.5 \mathrm{pu}$ and $k_{1}=1.5$ ( $+50 \%$ tolerance). Second, given a fixed tolerance in capacitance, the tolerance in actual power factor aggravates with reduced line current. In practice, this occurs when the wind turbine is operating under low speeds.

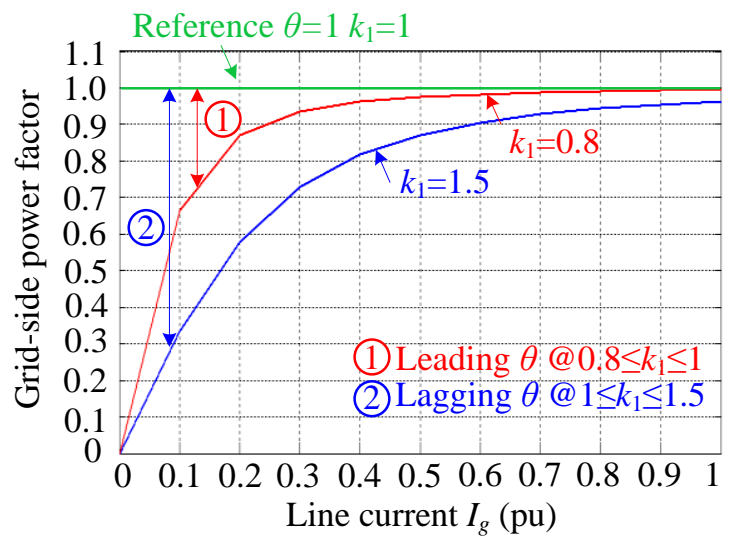

Figure 4-5 Tolerance in power factor. 
The capacitor voltage $V_{c}$ can be defined with the same way.

$$
V_{c}=\frac{j \omega L_{g} k_{2}\left(1-\omega^{2} C_{f} L_{g}\right) I_{g-U P F}-\omega^{2} L_{g} C_{f} V_{g} K_{2}\left(1-k_{1}\right)}{\left(1-\omega^{2} C_{f} L_{g} k_{1} k_{2}\right)}+V_{g}
$$

The first term in (4-8) represents the tolerance in capacitor voltage $V_{c}$, that it is too small that can be neglected compared with the second term, the grid voltage $V_{g}$. Thus, a well-balanced average input voltage can be obtained among CSCs.

In summary, the tolerance in the filter leads to significant tolerance in grid-side power factor which is not allowed by grid codes. The tolerance in average input voltage, on the other hand, can be negligible.

\subsubsection{Voltage distribution under power factor control}

To achieve UPF, a power factor control loop should be added to the conventional control scheme. In the following analysis, a two-module based system is taken as an example. And the capacitor voltage which is well maintained is not presented in the phasor diagram for simplicity.

Figure 4-6 shows the phasor diagram of the two-module system. Assuming the system is now operating under steady state by conventional scheme. By applying identical reference current, that is $I_{w_{-} r e f}\left(m_{a}\right.$ and $\alpha$ ), to the two modules, two different line currents ( $I_{g 1}$ and $\left.I_{g 2}\right)$ are generated. One $\left(I_{g 2}\right)$ is leading the grid voltage, while the other $\left(I_{g 1}\right)$ is lagging as shown in Figure 4-6 (a).

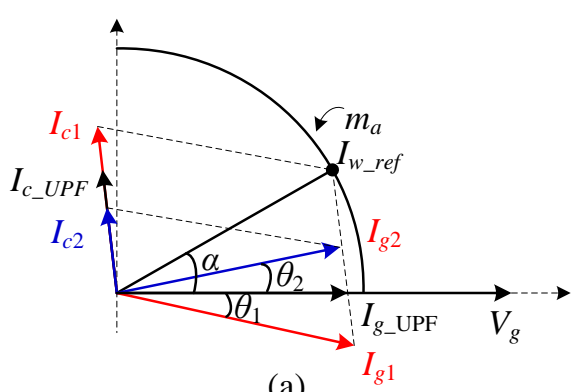

(a)

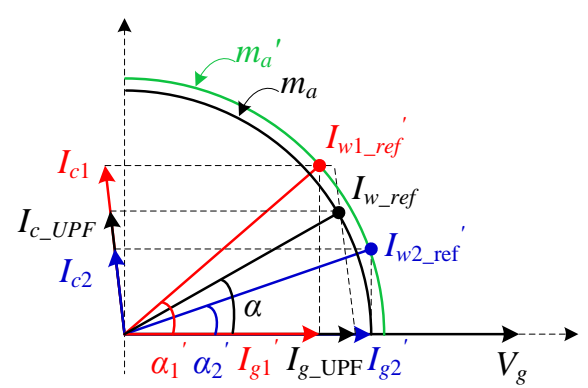

(b)

Figure 4-6 Phasor diagram under power factor control.

To achieve UPF, the delay angle $(\alpha)$ of the common reference current $I_{w 1}$ ref shown in Figure 46 (a) should be increased to compensate the lagging phase angle $\left(\theta_{1}\right)$ of the line current $I_{g 1}$ (Module \#1), while a reduced delay angle is needed to compensate a leading phase angle $\left(\theta_{2}\right)$ of the line current $I_{g 2}$ (Module \#1). After the regulation, the system will be operating under steady 
states again, but with a different operation point as shown in Figure 4-6 (b). The reference current for Module \#1 is now shifting from $I_{w_{-} r e f}$ to $I_{w 1 \_r e f}$ ', while it is shifted from $I_{w_{-} r e f}$ to $I_{w 2 \_r e f}{ }^{\prime}$ for Module \#2. $I_{w 1 \_r e f}{ }^{\prime}$ and $I_{w 2 \_r e f}{ }^{\prime}$ are with different delay angles, but sharing the same modulation index $\left(m_{a}^{\prime}\right)$.

The average input voltage for each module before and after power factor control is derived as follows. The input power $\left(P_{d c}\right)$ for the two-module system before the regulation shown in Figure 4-6 (a) is approximately equal to

$$
P_{i n}=V_{d c} I_{d c}=2 \cdot \sqrt{3 / 2} V_{L L} m_{a} \cos (\alpha)
$$

After the regulation, the system is operating with a different point as shown in Figure 4-6 (b)

$$
\begin{aligned}
& P_{i n}=\left(V_{d c 1}+V_{d c 1}\right) I_{d c}=\sqrt{3 / 2} V_{L L}\left\{m_{a}^{\prime} \cos \left(\alpha_{1}^{\prime}\right)+m_{a}^{\prime} \cos \left(\alpha_{1}^{\prime}\right)\right\} \\
& \alpha_{1}^{\prime} \approx \alpha-\theta_{1} \\
& \alpha_{2}^{\prime} \approx \alpha-\theta_{2}
\end{aligned}
$$

where $\theta_{1}$ and $\theta_{2}$ are calculated based on (4-7), and $\alpha$ is calculated based on (4-6).

Before and after the regulation, the input power is remaining the same. Combining (4-9) and (4-10) gives

$$
m_{a}^{\prime}=\frac{2 m_{a} \cos (\alpha)}{\cos \left(\alpha-\theta_{1}\right)+\cos \left(\alpha-\theta_{2}\right)}
$$

Based on (4) and (11), $V_{d 1}$ and $V_{d 2}$ are expressed as follows

$$
\begin{aligned}
& V_{d c 1}=\sqrt{3 / 2} V_{L L} m_{a}^{\prime} \cos \left(\alpha-\theta_{1}\right)=a \cdot\left(\cos \left(\alpha-\theta_{1}\right)\right) \\
& V_{d c 2}=\sqrt{3 / 2} V_{L L} m_{a}^{\prime} \cos \left(\alpha-\theta_{2}\right)=a \cdot\left(\cos \left(\alpha-\theta_{2}\right)\right)
\end{aligned}
$$

The resultant average input voltages $V_{d c 1}$ and $V_{d c 2}$ in the full operation range are shown in Figure 4-7 where the capacitor tolerances for Module \#1 and \#2 are $+50 \%\left(k_{1}=1.5\right)$ and $-20 \%$ $\left(k_{1}=0.8\right)$, respectively. Figure 4-7 shows that $V_{d c 1}$ is around 1.28 times of $V_{d c 2}$ under rated condition. Besides, the imbalance aggravates as reduced line current. Here, the given tolerance in filter parameters of the two modules is the critical case, one $\left(k_{1}=0.8\right)$ giving the maximum value $V_{d c 2}$, while the other $\left(k_{1}=1.5\right)$ giving the minimum value $V_{d c 1}$. The other average input voltage under other conditions will be random-distributed between them. Note that the number shown in 
Figure 4-7 may be slightly different with that in the following simulation and experiment. This is due to the approximation used in (4-10).

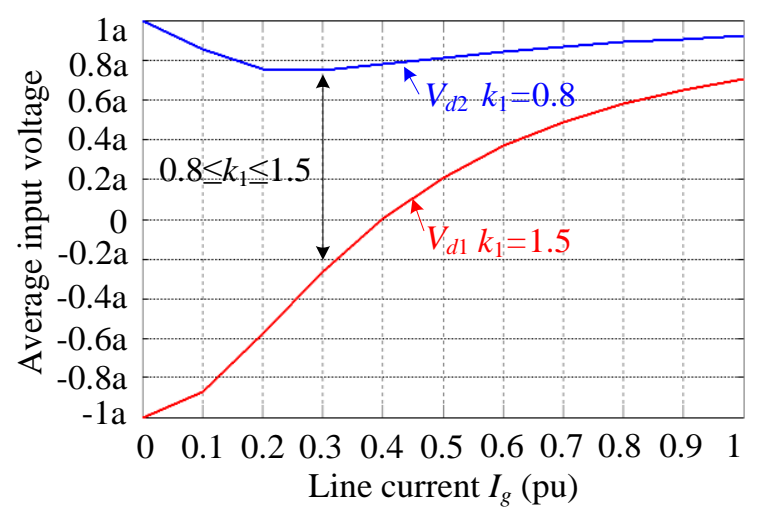

Figure 4-7 Input average voltage under UPF control.

In summary, three conclusions can be drawn based on above analysis. First, the instantaneous input voltage of each CSC is clamped by the grid voltage so that it is well balanced. Second, in the case of conventional scheme, the dc-link current control is ensued, the imbalance of average input voltage of each CSC can be neglected, while a significant tolerance in grid-side power factor occurs which is not allowed by grid codes. Third, UPF can be ensured by adding an additional power factor control to conventional scheme, though leading to great imbalance of average input voltage among CSCs. The one with higher average input voltage carries higher power that will be thermally overstressed. Thus, additional power balancing scheme is needed.

\subsubsection{Combined voltage balance control}

As analyzed above, to achieve both voltage/power balancing among CSCs and UPF at the grid side, different PWM reference currents are needed. Accordingly, a combined control scheme is proposed shown in Figure 4-8.

First, the conventional scheme (Figure 4-2) is entirely inherited. Besides, two additional control loops are added. One is the power factor control loop that helps ensure UPF at the grid side. The other is the voltage balance control that helps ensure voltage/power balance among CSCs. Each CSC is then controlled individually in the proposed scheme instead of sharing an identical control signal in conventional scheme.

The input modulation index $\left(m_{a}\right)$ and delay angle $(\alpha)$ is received in the way as same as that in conventional scheme. $V_{d c m_{-} r e f}$ is obtained by the captured wind power $\left(P_{d c}\right)$ and the dc-link 
current $\left(I_{d c}\right)$. The grid-side power factor $\theta_{\mathrm{n}}$ of each CSI is obtained by the phase locked loop. The output of the controller produces individual reference currents, $m_{a 1}$ and $\alpha_{1}$ are for Module \#1 and $m_{a \mathrm{~m}}$ and $\alpha_{\mathrm{m}}$ are for Module \#m, and then applied to each CSI. Once the input voltage balancing is achieved, the power of each CSI will be automatically balanced due to the series connection.

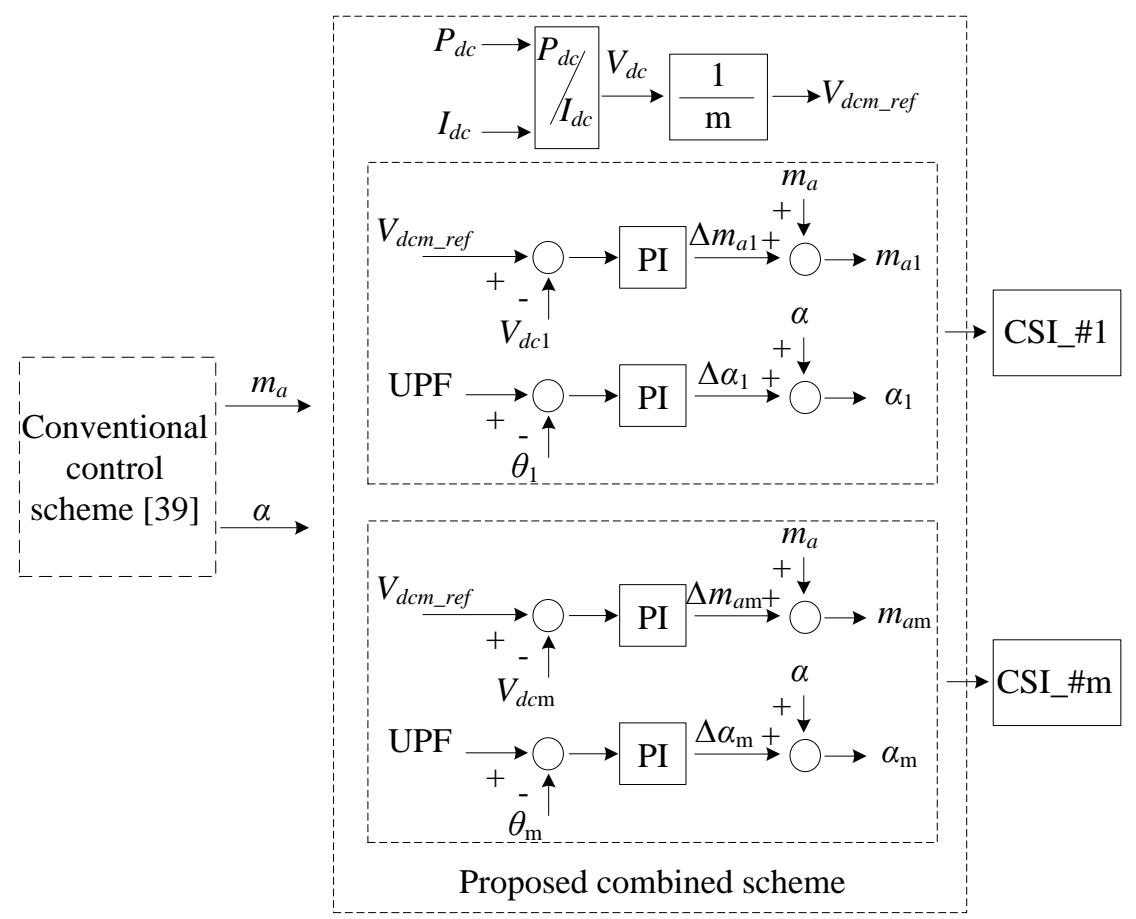

Figure 4-8 Proposed combined voltage balance control.

According to (4-4), the average input voltage of the CSC \#m after regulation $V_{d c_{-} n}$ can be expressed as

$$
V_{d c_{-} m}=\sqrt{3 / 2} V_{L L}\left(m_{a m}+\Delta m_{a m}\right) \cos \left(\alpha_{m}+\Delta \alpha_{m}\right)
$$

where $m_{a}$ and $\alpha$ are the common modulation index and delay angle for each CSC received based on conventional scheme, $\Delta m_{a \mathrm{~m}}$ comes from the average input voltage balancing control, and $\Delta \alpha_{\mathrm{m}}$ from the power factor control. For example, before time $t$, the system is operating under steady states where both voltage balancing and required power factor are ensured. At time $t$, the power factor control loop of one CSC is activated and a positive $\Delta \alpha_{\mathrm{m}}$ is obtained after the regulation. The new delay angle is then increased to $\alpha+\Delta \alpha_{\mathrm{m}}$, leading to a reduction in the average input voltage of the CSC according to (4-4). This therefore introduces an imbalance of the average input voltage and the voltage balancing control loop of the CSC is activated that a positive $\Delta m_{a m}$ is generated. Finally, the balancing is achieved again according to (4-13). 
The phasor diagram of the previous two-module system under the proposed scheme is shown in Figure 4-9. As shown in Figure 4-9, the reference current applied to the two CSCs based on conventional scheme is $I_{w_{-} r e f}\left(m_{a}\right.$ and $\left.\alpha\right)$. To ensure well-balanced average input voltage and gridside power factor, UPF, for example, the reference current for the CSC with a higher capacitor current $I_{c 1}$ shown in Figure 4-9 needs to shift from $I_{w_{-} r e f}\left(m_{a}\right.$ and $\left.\alpha\right)$ to $I_{w 1} r e f\left(m_{a 1}\right.$ and $\left.\alpha_{1}\right)$, and the reference current for the CSI with a lower capacitor current $I_{c 2}$ needs to shift from $I_{w_{-} r e f}\left(m_{a}\right.$ and $\alpha$ ) to $I_{w 2}$ ref $\left(m_{a 2}\right.$ and $\left.\alpha_{2}\right)$. During the process, both the modulation index and the delay angle are regulated. After the regulation, the same grid-side line current $I_{g_{-} U P F}$ is ensured for both CSIs as shown in Figure 4-9. Thus, both the average input voltage balancing and the required grid-side power factor of the two-module system are obtained.

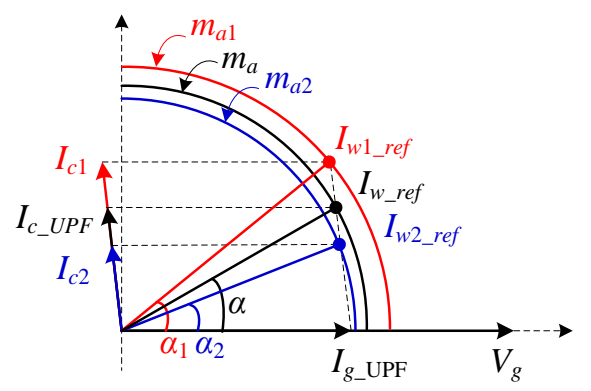

Figure 4-9 Phasor diagram based on the proposed combined control scheme.

\subsection{Performance investigation}

In this section, both simulations and lab-scale experiments are conducted to verify the effectiveness of the proposed scheme. Note that in practice, the maximum tolerance of capacitance can be well maintained within $\pm 20 \%$ [71], while the possible $+50 \%$ tolerance listed in some commercial products [71] may not be typically occurred. However, for such seriesconnected CSCs used for offshore wind power, the reliability is number one priority that the possibly most critical condition should be considered. Thus, to verify the effectiveness of the proposed separate scheme, the tolerance for capacitance is purposely set to be ranging from $-20 \%$ to $+50 \%$.

\subsubsection{Simulation investigation}

Figure 4-10 shows the simulated performance with and without the combined voltage balance control scheme. Before $t=1 \mathrm{~s}$, the system operates under conventional scheme. The average 
input voltages for Module \#1 and $\# 2\left(V_{d c 1}\right.$ and $\left.V_{d c 2}\right)$ are $4000 \mathrm{~V}$ and $3800 \mathrm{~V}$ with the resultant mismatch being around 5\% that is same as previous analysis. The power factor reference is set to unity that no reactive power is injected to the grid. The actual numbers, however, are with a different value. For example, the actual power factor for Module \#1 is -0.96 as shown in Figure 4-10 (c). At $t=1 \mathrm{~s}$, the power factor control is activated. The grid-side power factor is tracking its reference effectively. $V_{d c 1}$ and $V_{d c 2}$, on the other hand, start to diverge quickly and finally reach to a steady state with $V_{d c 1}=3450 \mathrm{~V}$ and $V_{d c 2}=4350\left(V_{d c 1}=1.27 V_{d c 2}\right)$. At $t=2 \mathrm{~s}$, the proposed scheme is activated. $V_{d c 1}$ and $V_{d c 2}$ start to converge quickly, while the power factor is remaining unity. During the process, the dc-link current $I_{d c}$ is controlled to be constant as shown in Figure 4-10 (a).
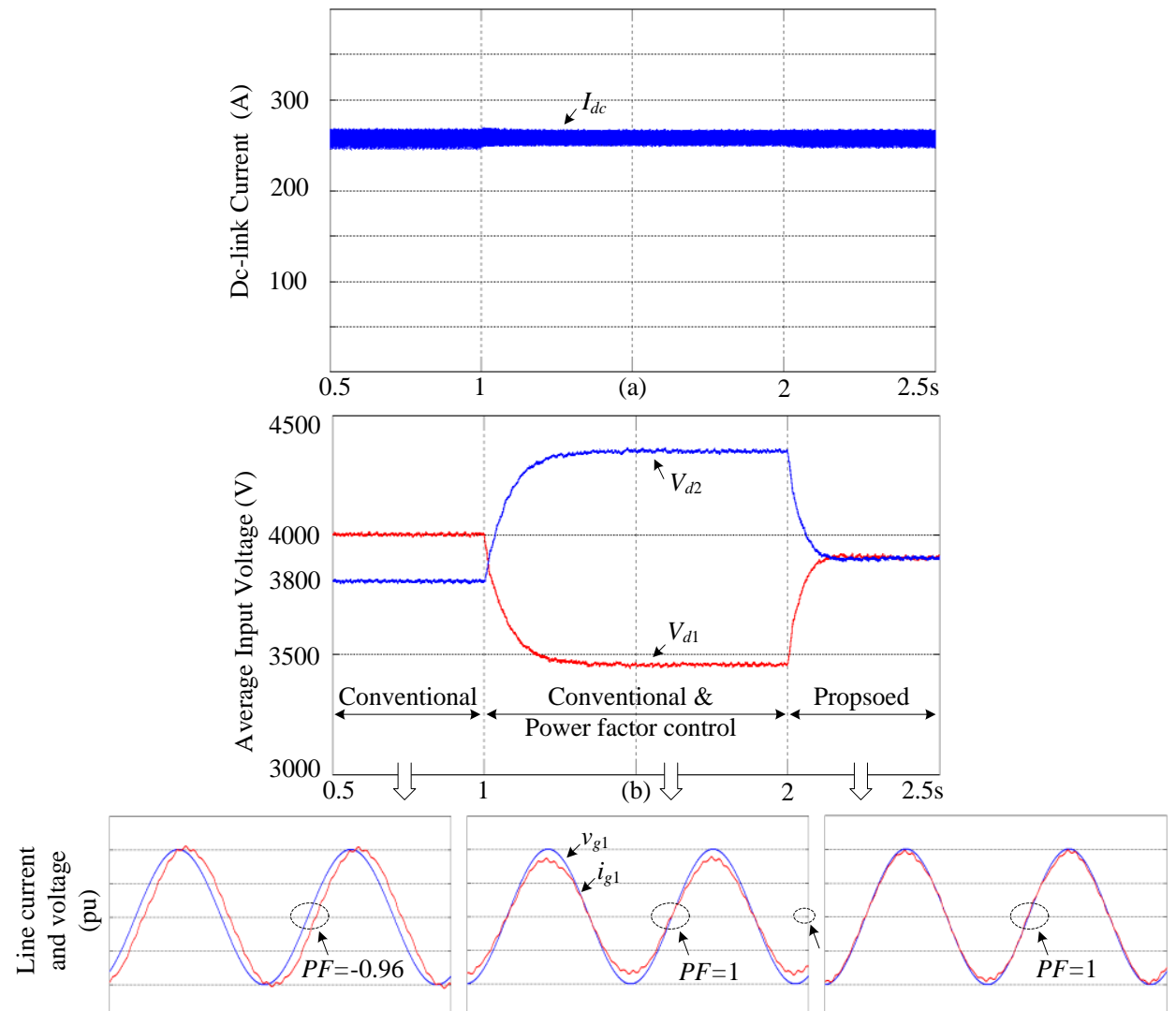

(c)

Figure 4-10 Simulated performance with and without voltage balance control.

Figure 4-11 shows the simulated performance under stepped dc-link current. This is to emulate the stepped wind speed in practice. Figure 4-11 shows that the dc-link current control, the gridside power factor control, and average input voltage balancing control of the two CSCs are ensured. 

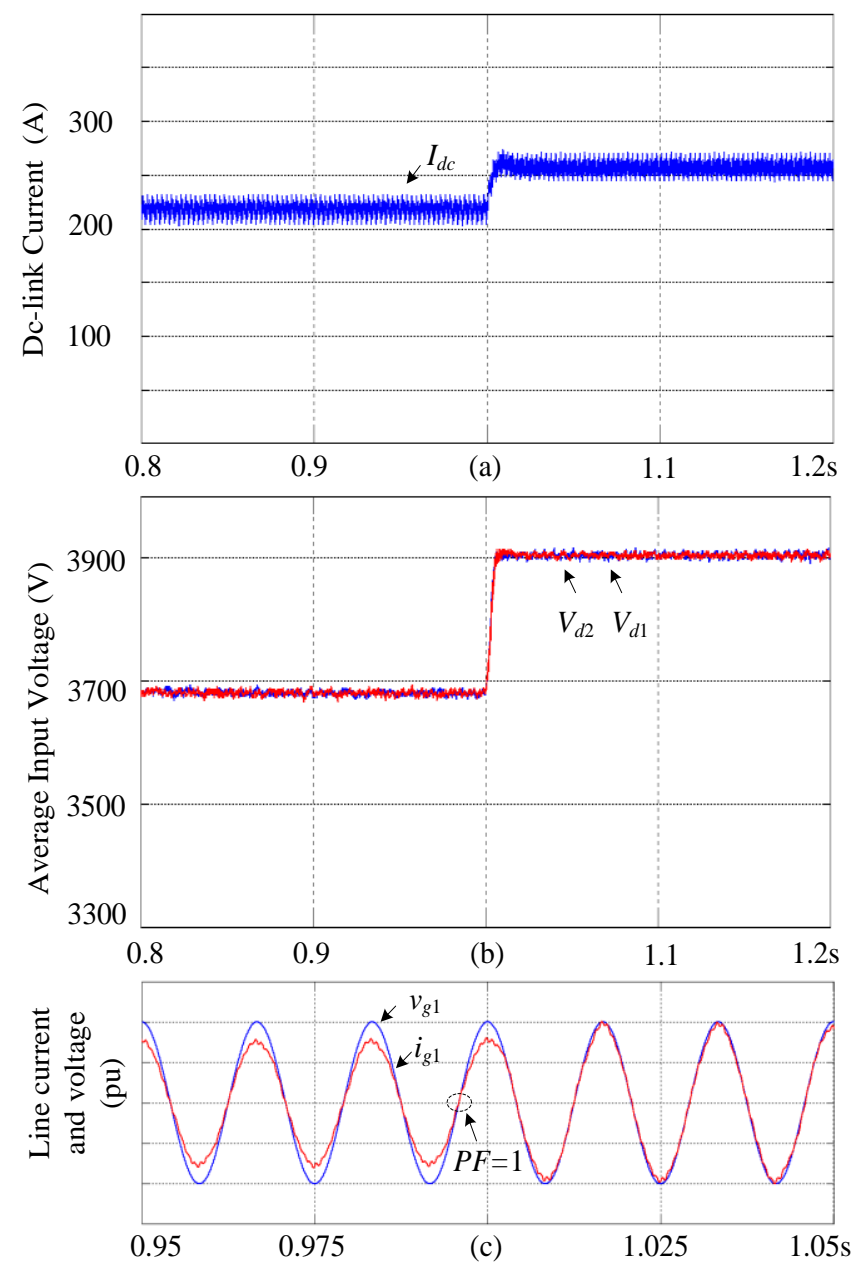

Figure 4-11 Simulated performance under stepped dc-link current.

\subsubsection{Experimental verification}

Figure 4-12 shows the experimental performance with and without the combined voltage balance control scheme. Note that in the experiment, the inverter-side line-to-line voltage of the variac transformer which is connected to the grid is measured. Thus, in the following, the displacement between the line current and the phase grid is equal to $\theta^{\prime}-30^{\circ}$ where $\theta^{\prime}$ is the displacement between the line current and the line-to-line voltage.

When only conventional scheme is applied, average input voltages $V_{d c 1}$ and $V_{d c 2}$ are $92 \mathrm{~V}$ and $87 \mathrm{~V}$ with the resultant difference between them being around 5\%. The power factor reference $\theta_{\text {ref }}$ is set to unity, while the actual number is different. Figure 4-12 (c) shows injected line current $i_{g 1}$ and line-to-line voltage $v_{a b}$ of Module \#1. The actual power factor is -0.96 that is same as that in the previous analysis. The imbalance of average input voltage of the two modules 
aggravates when the reference power factor is obtained with the help of power factor control as shown in Figure 4-12 (b). $V_{d c 1}$ is decreasing from $92 \mathrm{~V}$ to $78 \mathrm{~V}$, while $V_{d c 2}$ is increasing from 87 $\mathrm{V}$ to $100 \mathrm{~V}$ with the resultant mismatch reaching to $28 \%$. When the proposed separate scheme is applied, $V_{d c 1}$ and $V_{d c 2}$ start to converge quickly and the power factor is remaining unity. During the whole process, the dc-link current $I_{d c}$ is well controlled as shown in Figure 4-12 (a).

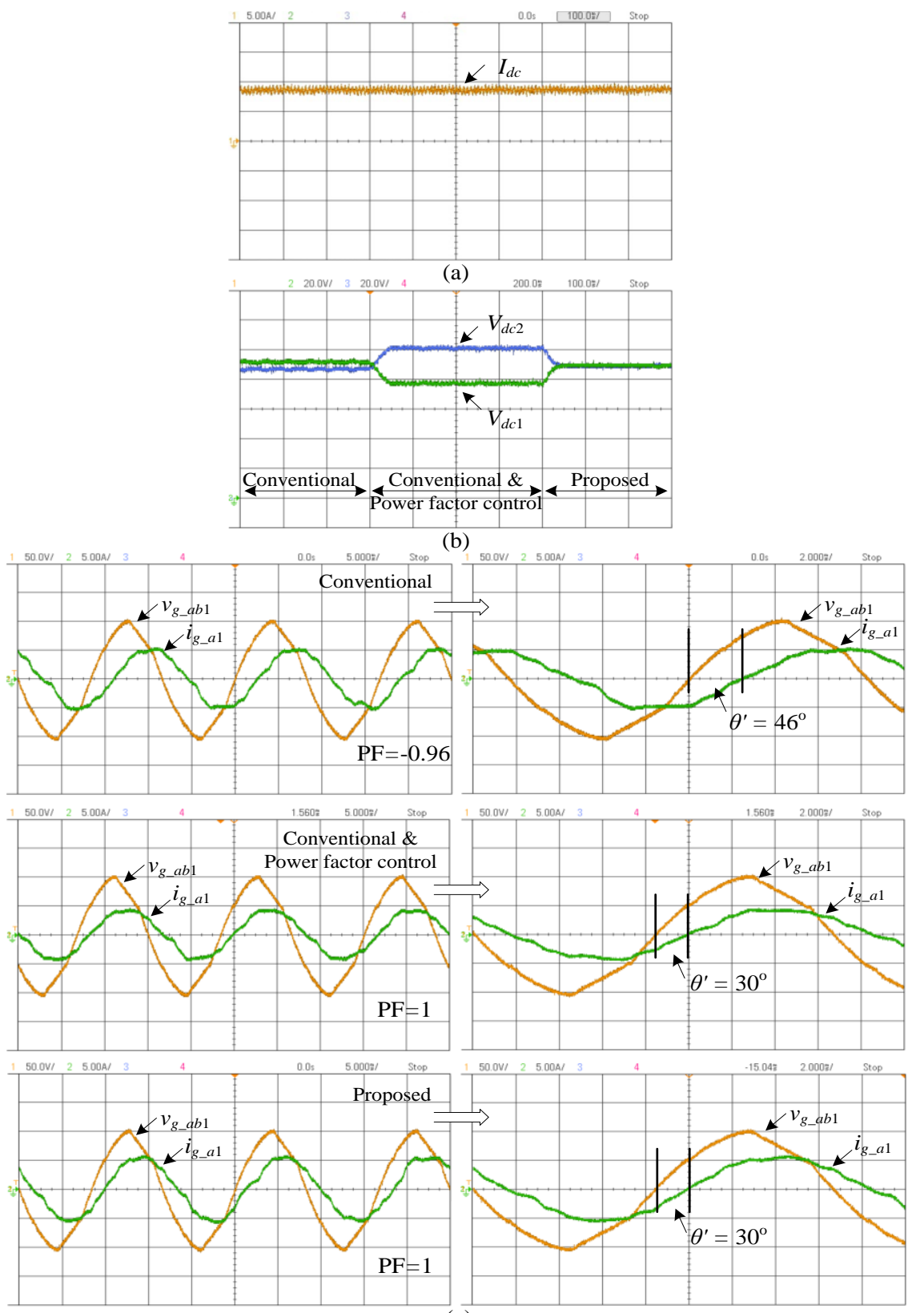

(c)

Figure 4-12 Experimental performance with and without voltage balance scheme. 
Figure 4-13 shows the experimental performance under stepped dc-link current. The average input voltages of the two CSCs are well balanced and the grid-side power factor is maintained at unity.

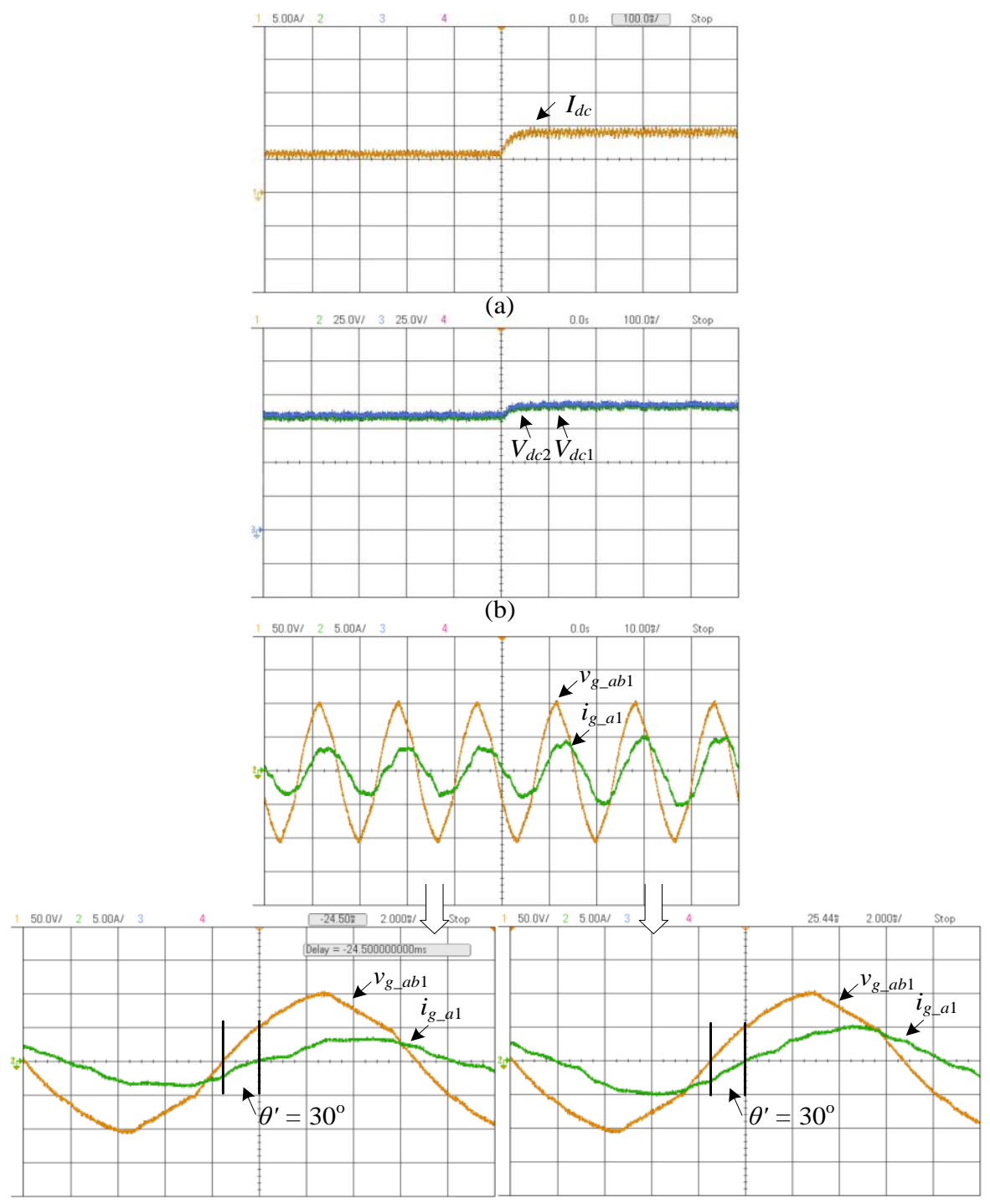

(c)

Figure 4-13 Experimental performance under stepped dc-link current.

\subsection{Conclusions}

In this chapter, the power distribution among grid-side series-connected MV CSCs is investigated. First, the power imbalance among series-connected CSCs is unveiled where the tolerance in filter parameters is the dominated factor. Second, conventional scheme gives a small mismatch of power, but produces a significant tolerance in power factor which is not allowed by 
the grid codes. To meet grid codes, an additional power factor control has to be added. The resultant imbalance, however, aggravates greatly. Third, a combined control scheme is proposed. Compared with conventional scheme, two additional control loops are added. One is the voltage balance control used to ensure equal voltage/power distribution among CSCs. The other is the power factor control employed to guarantee the grid-side power factor. In summary, the combined scheme ensures dc-link current control, grid-side power factor control, and power balancing among series-connected CSCs simultaneously.

Both simulation and experiments are provided to verify the effectiveness of the proposed scheme. 


\section{Chapter 5}

\section{DC-link Current Control of Offshore Wind Farm with Bipolar HVDC System}

The proposed CSC-based series-connected offshore wind farm features smaller size and weight as no offshore substation and low-frequency transformers are used. However, a big challenge exists for the system, that is the high insulation requirement. A CSC-based configuration is normally operating under monopolar mode. The resultant maximum insulation requirement is the full transmission voltage, leading significant challenge to the whole system in terms of cost and reliability. Bipolar operation gives lower insulation requirement. However, the dc-link current control is a main concern for the CSC-based configuration operating under bipolar mode. There are two equivalent paths for the dc-link current and the dc-link current control directly determines the efficiency and flexibility of the system. Therefore, the objectives of this chapter are to investigate the performance of the bipolar operation and propose an optimal dc-link current control.

This chapter starts with a brief introduction of conventional dc-link current, followed by the proposed optimal dc-link current control under bipolar mode. Equivalent circuits for monopolar and bipolar modes are derived respectively. Compared with monopolar mode, bipolar mode gives lower insulation requirement, thus contributing to lower cost and higher reliability. Compared with conventional dc-link current control, the proposed one features higher efficiency and flexibility. Both simulation and experimental results are provided to verify the performance of the system under bipolar mode and the effectiveness of the optimal dc-link current control.

\subsection{Monopolar operation}

Figure 5-1 shows the CSC-based series-connected offshore wind farm configuration. In the offshore part, a number of turbine units are connected in series to reach an HVDC level. In the onshore part, a number of MV CSCs are connected in series to form a centralized dc/ac converter. The step-up multi-winding transformers are employed to connect the converters to the grid, 
providing isolation and grid integration. A dc-link inductor, that is $L_{d c}$, is shared by offshore and onshore converters.

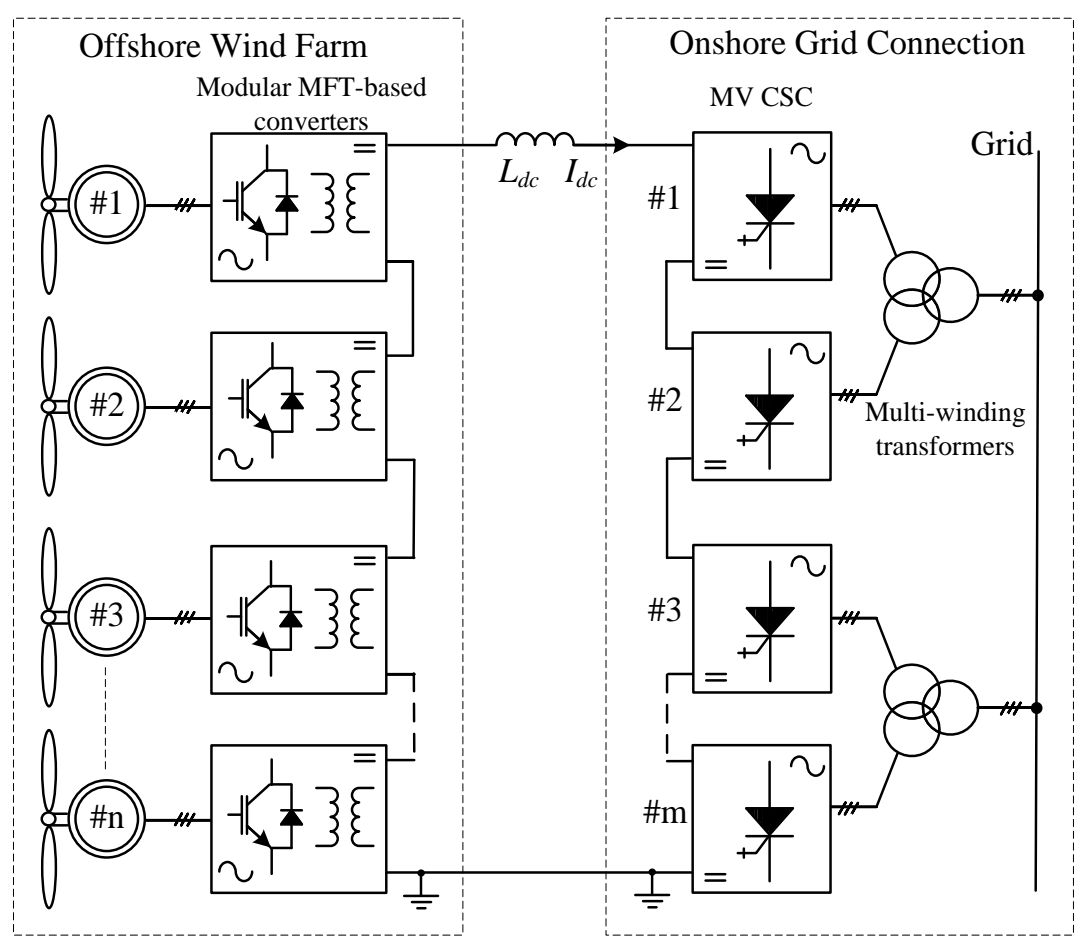

Figure 5-1 CSC-based offshore wind farm with monopolar HVDC system.

CSC-based configuration is normally operating under monopolar mode as shown in Figure 5-1. However, one significant challenge exists, that is the high system insulation level, leading to higher cost, lower reliability, and limited power capacity.

The wind generator that is farthest from the grounding point must be capable of withstanding the full transmission voltage. To tackle this issue, a couple of methods are proposed as follows [72], [73].

(a) insulate the generator winding, and offshore converter for high potential (the full transmission voltage) to the ground, or

(b) insulate the wind turbine tower for high potential (the full transmission voltage) to the ground and keep the nacelle on high potential, or

(c) using transformers.

Among the above methods, the transformer-based solution seems more reasonable in practice. Ref. [40] employed a low-frequency high-power transformer between generator and the front- 
end CSC that generators with regular insulation levels can be used. However, this low-frequency transformer is heavy and bulky increasing burden on offshore construction as the space either in the nacelle or in the tower of the wind turbine is limited [6]. To solve this issue, a modular MFTs-based configuration is presented in this work. It features smaller size and weight which is particularly important for offshore applications. However, the technology for such a high-power MFT with the maximum insulation level being around an HVDC level, $300 \mathrm{kV}$ and higher, for example, is immature [45].

First, such a high insulation level produces significant costs on the high-voltage isolated transmission cables as well as the modular MFTs and converters that all with high-voltage isolation requirement. Second, the reliability is reduced as increased insulation requirement. Third, the power capacity of the series-connected system under monopolar mode is limited. More turbine units need be connected in series to reach a higher HVDC level and achieve higher transmission capacity. In practice, this is impractical mainly due to the limitation of the increased insulation requirement.

In summary, the CSC-based series-connected offshore wind farm with monopolar HVDC system results in a full transmission insulation requirement, leading to higher cost, lower reliability, and limited power capacity.

\subsection{Bipolar operation}

Bipolar operation is typically used in VSCs where the insulation of the system is half of the dclink voltage. Following this, the CSC-based series-connected configuration under bipolar mode is given in Figure 5-2 [74].

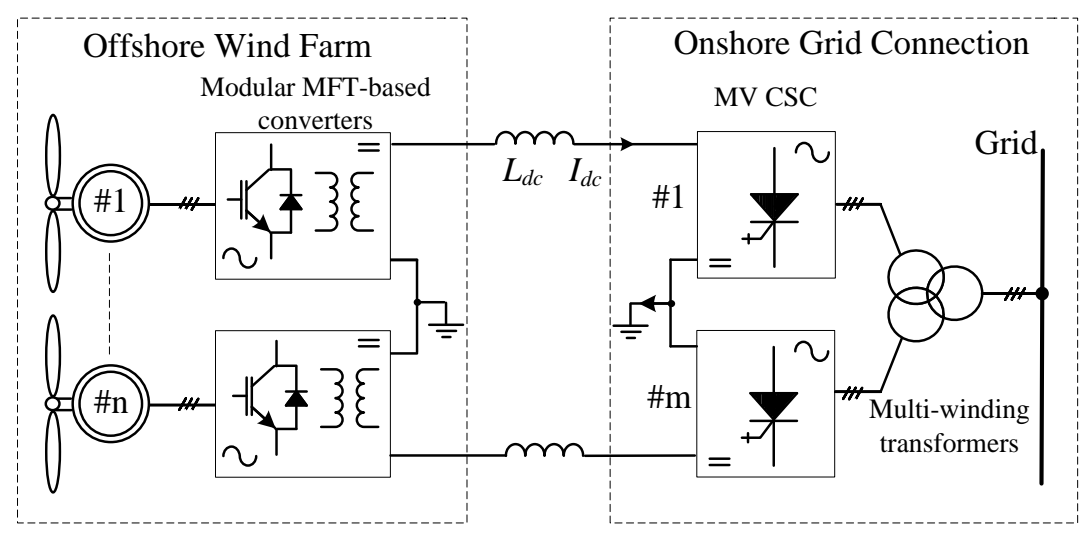

Figure 5-2 CSC-based offshore wind farm with bipolar HVDC system. 
Bipolar mode lowers the insulation level. Given a transmission voltage level in monopolar mode, that is $300 \mathrm{kV}$, for example, the resultant insulation requirement under bipolar mode is now reduced to $150 \mathrm{kV}$, giving significant cost saving as well as higher reliability. In addition, a bigger transmission capacity can be obtained by extending the bipolar system to a multi-port system as shown in Figure 5-3. In the case of monopolar system, however, this can only be achieved by connecting more modules in series which is impractical as previously mentioned. In summary, the CSC-based configuration with bipolar operation mode features lower insulation requirement, giving lower cost, higher reliability, and higher transmission capacity.

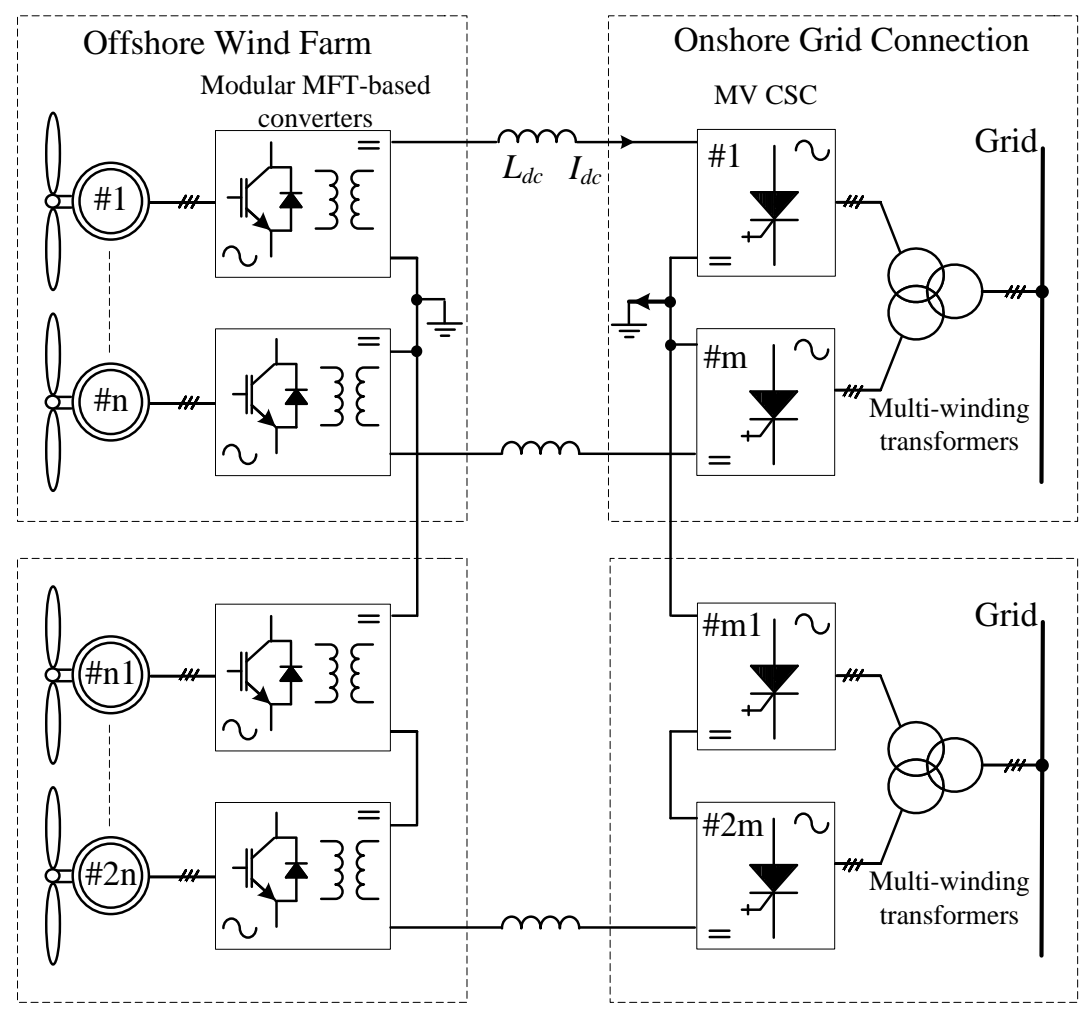

Figure 5-3 Multi-port CSC-based offshore wind farm.

However, a technical challenge exists for the system operating under bipolar mode, that is the dc-link current control. Figure 5-4 illustrates the equivalent circuits of the system under monopolar and bipolar modes, respectively. Monopolar mode has one current path that the dclink current is flowing through all the converters. Bipolar mode, on the other hand, has two current paths. The dc-link current control proposed for monopolar mode can be used in bipolar mode, though with the penalty of lower efficiency which will be illustrated in the following. Thus, an optimal dc-link current control bringing high efficiency for the bipolar system is needed. 

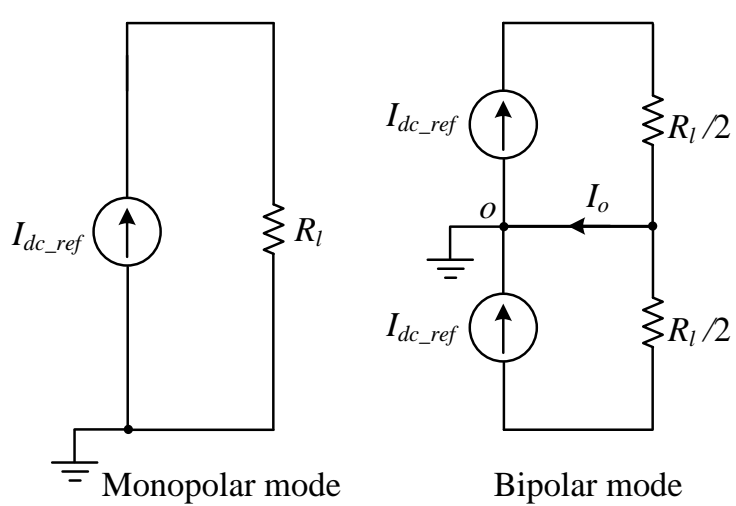

Figure 5-4 Path for dc-link current under monopolar and bipolar modes.

\subsection{Optimal dc-link current control}

The dc-link current control plays a critical role in offshore wind farm operations. In general, two constraints are set for the dc-link current control. First, the dc-link reference current should be equal or greater that the maximum value between generator- and grid-side determined minimum dc-link reference currents. A minimum dc-link current is required to achieve MPPT for offshore turbine-generator converter, below which MPPT cannot be obtained. Similarly, a minimum number is required for grid-side converter to ensure power factor control, UPF, for example. Thus, the final dc-link reference current should be equal or greater than the two "minimum values" to ensure all control objectives simultaneously. Second, the dc-link current should be maintained as small as possible to lower operation losses. Unlike a VSC-based wind farm where the dc-link voltage is controlled at a constant value, the dc-link current in a CSCbased wind farm can either be controlled as constant or varied according to the variation in the input power. The fixed dc-link current operation gives a faster dynamic response but at the expense of high system losses during low wind speeds [2], while a variable dc-link current greatly reduces the system losses with the penalty of low dynamic performance [2]. Fast dynamic response, however, is not mandatory in wind energy system because the large inertia of the turbine-generator system gives slow change in the output power. On the other hand, a high efficiency is much more preferred. In summary, a proper "minimum" dc-link current reference is the key point for the whole system.

\subsubsection{DC-link reference current determination}

(a) Generator-side reference current 
The primary objective for the generator-side converter is to obtain the maximum power input from varying wind speeds. This can be achieved by regulating the modular MFT-based converter as shown in Figure 2-2. A simplified circuit diagram of the offshore converter interfacing the generator unit \#n is shown in Figure 5-5. The PMSG is simplified with a voltage source $\left(e_{s}\right)$ with a finite source inductance $\left(L_{g}\right)$, representing the back-EMF and the synchronous inductance, respectively. The modular MFT-based converter is simplified with an ideal Buck converter. The load at the dc link is considered as a current source.

Due to the presence of a large input line inductance, the current commutations in the rectifier are not instantaneous and will cause voltage drops at the dc output. The commutation angle as well as the resultant voltage drop depends on the generator inductance $L_{s}$ and the dc-link current $I_{d c}$. If the commutation angle is less than $60^{\circ}$, the average dc output voltage $V_{d c_{-} \mathrm{n}}$ and commutation angle $\delta$ can be calculated as follows [75],

$$
\begin{gathered}
V_{d c_{-} n}=\frac{3 \sqrt{2}}{\pi} E_{s, L L}-\frac{3}{\pi} \omega_{s} L_{s} I_{d c} \\
\cos (\delta)=1-\frac{\sqrt{2} \omega_{s} L_{s} I_{d c}}{E_{s, L L}}\left(0 \leq \delta \leq 60^{\circ}\right)
\end{gathered}
$$

where $E_{s, L L}$ is the $r m s$ value of the line-to-line back-EMF $e_{s} ; w_{s}$ is the optimum electrical speed of the PMSG under MPPT.

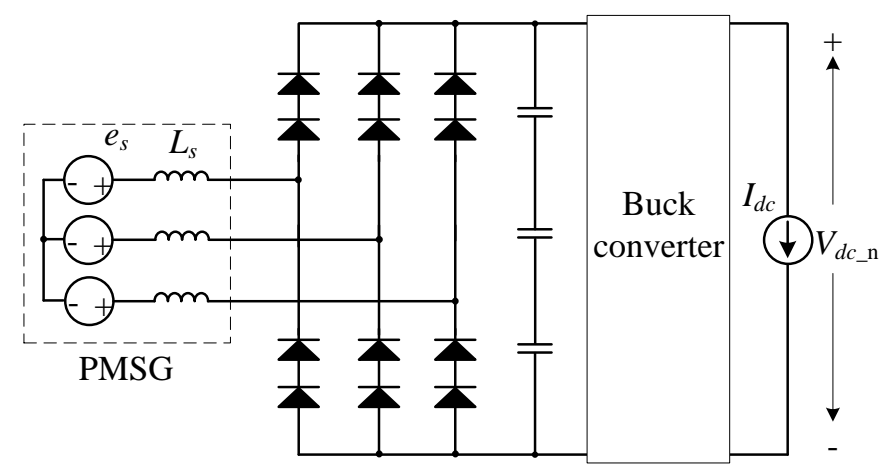

Figure 5-5 A simplified modular MFT-based converter for unit \#n.

It can be observed from (5-1) and (5-2) that the longer time the commutation takes the more voltage drops the dc output will suffer. If the commutation angle increases and exceeds $60^{\circ}$, the decrease of the dc voltage will be more rapid until it drops to zero. Rectifier input power factor 
will decrease as well and the generator stator winding current will increase substantially. This operation mode is unreasonable and should be avoided.

Assuming the captured wind power $P_{g e_{-} \mathrm{n}}$ is transferred to the dc side $P_{d c_{-} \mathrm{n}}$ with the loss being neglected, there is,

$$
P_{d c_{-} n}=V_{d_{-} n} I_{d c}=P_{g e_{-} n}
$$

Combining (5-1) and (5-3) gives,

$$
P_{g e_{-} n}=\left(\frac{3 \sqrt{2}}{\pi} E_{s, L L}-\frac{3}{\pi} \omega_{s} L_{s} I_{d c}\right) I_{d c}
$$

The generator-side dc-link current is therefore calculated as follows.

$$
I_{d c}=\frac{3 \sqrt{2} E_{s, L L} \pm \sqrt{18 E_{s, L L}{ }^{2}-12 \pi \omega_{s} L_{s} P_{g e_{-} n}}}{6 \omega_{s} L_{s}}
$$

To achieve MPPT, a real solution of $I_{d c g}$ should be found from (5-5). Accordingly, the following parameter constraint for the synchronous generator needs to be satisfied.

$$
E_{s, L L}^{2} \geq \frac{2 \pi}{3} \omega_{s} L_{s} P_{g e_{-} n}
$$

Based on (5-5) and (5-6), two real solutions are found for the dc-link current. The smaller one is selected while the larger one is unreasonable in practice as it cannot satisfy the prerequisite given in (5-2). In addition, the lost duty cycle of the modular MFT-based converter due to the presence of the transformer leakage inductance should be compensated. Here, the maximum lost duty cycle is set to 0.1 .

The resultant dc-link reference current determined by generator-side converter is

$$
I_{d c_{-} g e n}=\frac{3 \sqrt{2} E_{s, L L}-\sqrt{18 E_{s, L L}^{2}-12 \pi \omega_{s} L_{s} P_{g e_{-} n}}}{6 \omega_{s} L_{s} \cdot 0.9}
$$

Figure 5-6 shows the minimum dc-link reference current determined by the generator-side converter. First, the curves are given under different magnetic fluxes, ranging from 0.8 to $1.2 \mathrm{pu}$. It's reasonable that when the generator terminal voltage drops as the magnetic flux reduces, the dc-link reference current is increasing accordingly in order to obtain the same amount of power in 
the dc-link. Second, a higher synchronous inductance gives a slightly larger reference current.

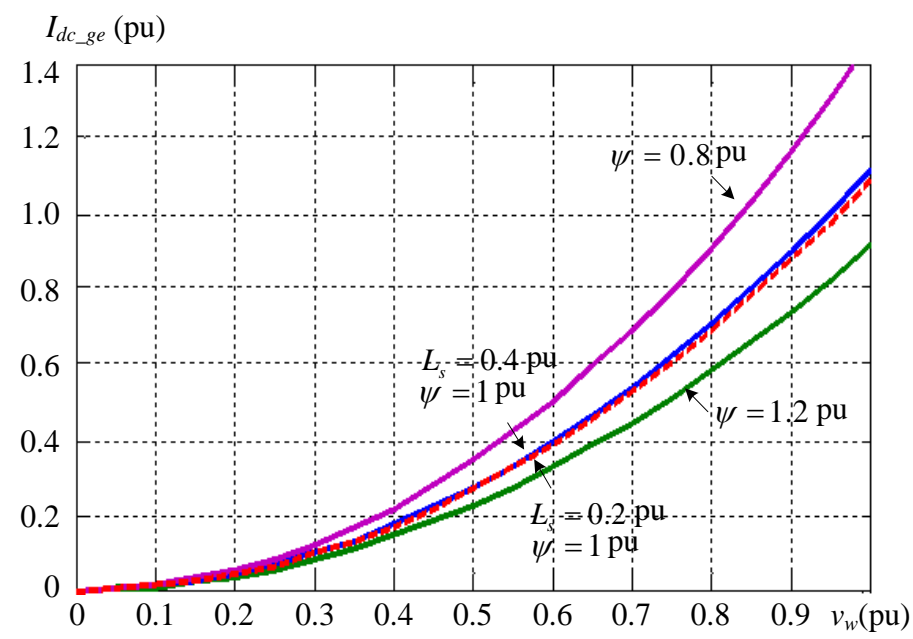

Figure 5-6 Reference dc-link current determined by generator-side converter.

(b) Grid-side reference current

The total available wind power of the wind farm $\left(P_{W F}\right)$ is expressed as follows.

$$
P_{W F}=\sum_{i=1}^{n}\left(P_{g e_{-} i}\right)
$$

The grid-voltage phase-locked loop is employed to generate a noise-free synchronous angle $\theta_{g}$ and angular frequency $\omega_{g}$. Assuming a lossless system, the total available wind power $\left(P_{W F}\right)$ is equally shared by each grid-side converter. Based on the reactive power demand $\left(Q_{c m d}\right)$ from the system operator, the dc-link reference current for onshore-side converter is derived as follows.

$$
\begin{gathered}
P_{W F}=m \cdot \frac{3}{2} V_{s d} i_{s d} \\
Q_{c m d}=m \cdot\left(-\frac{3}{2}\right) V_{s d} i_{s q}
\end{gathered}
$$

where $V_{s d}$ and $V_{s q}, i_{s d}$ and $i_{s q}$ are the $d$ - and $q$-axis components of the grid voltage and current, respectively.

The reference current of the CSC $i_{w d_{-} r e f}$ and $i_{w q_{-} r e f}$ can be derived as follows [38]: 


$$
\left\{\begin{array}{l}
i_{w d_{-} r e f}=i_{s d_{-} r e f}+i_{c d}=i_{s d_{-} r e f}-\omega_{g} C_{f} V_{c q} \\
i_{w q_{\_} r e f}=i_{s q_{-} r e f}+i_{c q}=i_{s q_{-} r e f}+\omega_{g} C_{f} V_{c d}
\end{array}\right.
$$

where $i_{s d_{-} r e f}$, and $i_{s q_{-} r e f}$ are the references of the $d$ - and $q$-axis components of the grid current. $i_{c d}$ and $i_{c q}$ and $V_{c d}$ and $V_{c q}$ are $d$ - and $q$-axis components of the capacitor current and the voltage respectively. These variables can be expressed as follows [38]:

$$
\left\{\begin{array}{l}
V_{c q}=R_{g} i_{s d}+V_{s d}-\omega_{g} L_{g} i_{s q} \\
V_{c q}=R_{g} i_{s q}+\omega_{g} L_{g} i_{s d}
\end{array}\right.
$$

where $L_{g}$ and $R_{g}$ represent the grid-side line inductance and resistance, respectively.

With the modulation index $m_{a}$ being set to its maximum value, that is 1 , to lower conduction losses [2], the magnitude of the minimum dc-link current $I_{d c_{-} g r}$ determined by the grid-side converters can be derived based on (5-8)-(5-12):

$$
I_{d c_{-g r}}=\sqrt{\left\{\left(1-\omega_{g}^{2} L_{g} C_{f}\right)\left(\frac{2}{3} \frac{P_{W F}}{m V_{s d}}\right)-\omega_{g} R_{g} C_{f}\left(\frac{2}{3} \frac{Q_{c n d}}{m V_{s d}}\right)\right\}^{2}+\left\{\left(1-\omega_{g}{ }^{2} L_{g} C_{f}\right)\left(\frac{2}{3} \frac{Q_{c m d}}{m V_{s d}}\right)+\omega_{g} C_{f} V_{s d}+\omega_{g} R_{g} C_{f}\left(\frac{2}{3} \frac{P_{W F}}{m V_{s d}}\right)\right\}^{2}}
$$

Again, note that, in the case of a wind farm, the reactive power required by grid codes is normally achieved by adding extra reactive support equipment at the plant level, such as SVC and STATCOM. Thus, only UPF needs be considered. The grid-side line resistance $R_{g}$ is too small that can be neglected. Then the grid-side minimum reference current is simplified as

$$
I_{d c_{-} g r}=\sqrt{\left.\left\{\left(1-\omega_{g}^{2} L_{g} C_{f}\right)\left(\frac{2}{3} \frac{P_{W F}}{m V_{s d}}\right)\right\}^{2}+\left(\omega_{g} C_{f} V_{s d}\right)\right)^{2}}
$$

\section{(c) Final reference current}

The dc-link currents determined by generator- and grid-side converters in the full wind speeds are illustrated in Figure 5-7. As mentioned earlier, the larger one is selected to be the final dc-link reference current. Note that all the reference currents shown in Figure 5-6 and Figure 5-7 are in a per-unit system. The base dc-link current is the minimum current determined by the grid-side converter under the condition of UPF as per (5-13).

Two conclusions are drawn from Figure 5-7. First, smaller filter capacitors $\left(C_{f}\right)$ output lower minimum dc-link reference currents. The grid-side CSC has a three-phase ac capacitor, which 
absorbs considerable amount of leading current. The leading current needs to be compensated by the converter if UPF is required at the grid connection point. In low wind speed ranges (0-0.4pu), the captured real power is very low (the captured wind power is proportional to the cube of the wind speed) and the dc-link current is mainly determined by the reactive current in the capacitors, resulting in almost constant values. As the wind speed goes up, the grid-side dc-link current requirements rise as well as the active current increases. Note that the output capacitor filter $C_{f}$ is normally ranging from $0.15 \mathrm{pu}$ to $0.3 \mathrm{pu}$ for a dual-bridge CSC thanks to the grid-side phaseshifted multi-winding transformers [22].

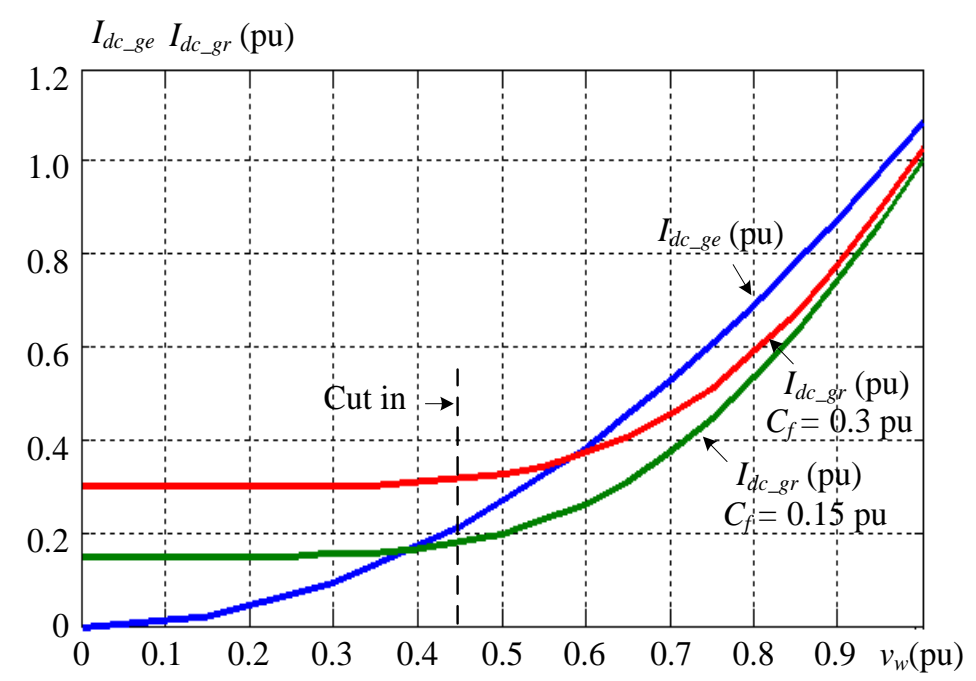

Figure 5-7 Reference dc-link current determined by generator- and grid-side converters.

Second, it is the generator-side rather than the grid-side converter determining the final dc-link reference current of the wind farm in the main operation range. For example, in the case of $C_{f}=$ $0.15 \mathrm{pu}$, the generator-side reference current is higher than that of the grid-side one in the full operation range, and thus is selected to be the final reference current of the wind farm. A similar result is also found in the case of $C_{f}=0.3 \mathrm{pu}$, but mainly in high operation ranges, that is ranging from 0.58 to $1 \mathrm{pu}$.

\subsubsection{Optimal dc-link current control}

When the CSC-based series-connected system operates under monopolar mode shown in Figure 5-1, the currents through all individual modules are same. However, the wind speed at each turbine cannot be guaranteed to be identical. The inconsistency of wind speeds leads to different dc-link reference currents for each unit. To ensure all control objectives, conventional 
scheme picks the maximum value among all to be the final dc-link reference current as shown in Figure 5-8 [40].

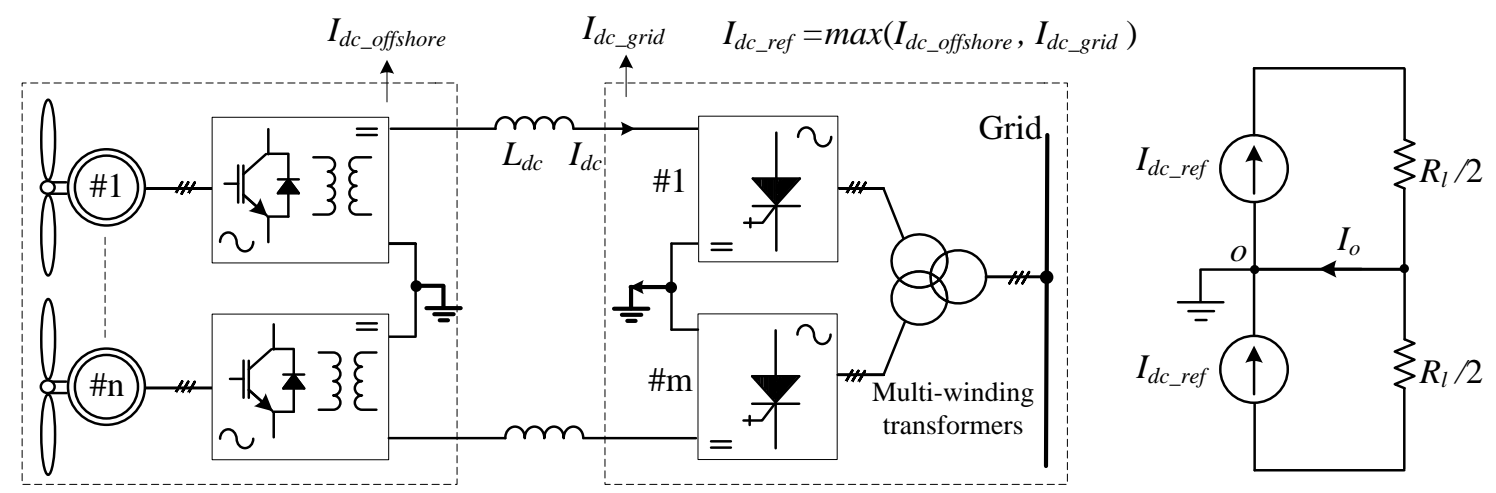

Figure 5-8 Bipolar offshore wind farm with conventional dc-link current control.

The principle of the bipolar operation using conventional scheme is given in Figure 5-8. $I_{d c_{-} o f f s h o r e}$ is the maximum current among all offshore generator-side converter determined reference currents, while $I_{d c_{-} g r i d}$ is the reference current determined by the onshore grid-side CSCs. The larger one is selected to be the final dc-link reference current of the whole wind farm $I_{d c_{-} r e f}$. Ideally, the current on the earth path $\left(I_{o}\right)$ is zero. $R_{l}$ represents the equivalent load of the whole system. Such a dc-link reference current can ensure all the control objectives of the wind farm, but with a penalty of high operation losses which will be discussed in the following.

An optimized dc-link current control with higher efficiency is proposed. Instead of picking the maximum value among all reference currents as shown in Figure 5-8 to be the final dc-link reference current of the whole system, two reference currents are separately selected for the positive and negative links of the bipolar system. Accordingly, the bipolar system is controlled as two monopolar systems with independent dc-link current control. The resultant equivalent circuit is shown in Figure 5-9. $R_{l_{-} 1}$ and $R_{l_{2} 2}$ represent the equivalent loads for upper and lower parts of the bipolar system. $I_{d c_{-} r e f 1}$ and $I_{d c_{-} r e f 2}$ are defined as follows.

$$
\begin{gathered}
I_{d c_{-} r e f 1}=\max \left(I_{d c_{-} g e 1,} I_{d c_{-} g e 2, \cdots,} I_{d c_{-} g e p,} I_{d c_{-} g r 1}\right) \\
I_{d c_{-} r e f 2}=\max \left(I_{d c_{-} g e(p+1),} I_{d c_{-} g e(p+2), \cdots,} I_{d c_{-} g e m,} I_{d c_{-} g r 2}\right)
\end{gathered}
$$

where $\mathrm{p}$ is the number of the turbine-generator units with $\mathrm{p}=\mathrm{m} / 2 . I_{d c_{-} g r 1}$ and $I_{d c_{-} g r 2}$ are calculated based on (5-14).

Such an optimized dc-link current control can both ensure all the control objectives achieved in 
monopolar system and improve the overall efficiency and the flexibility of the whole system.

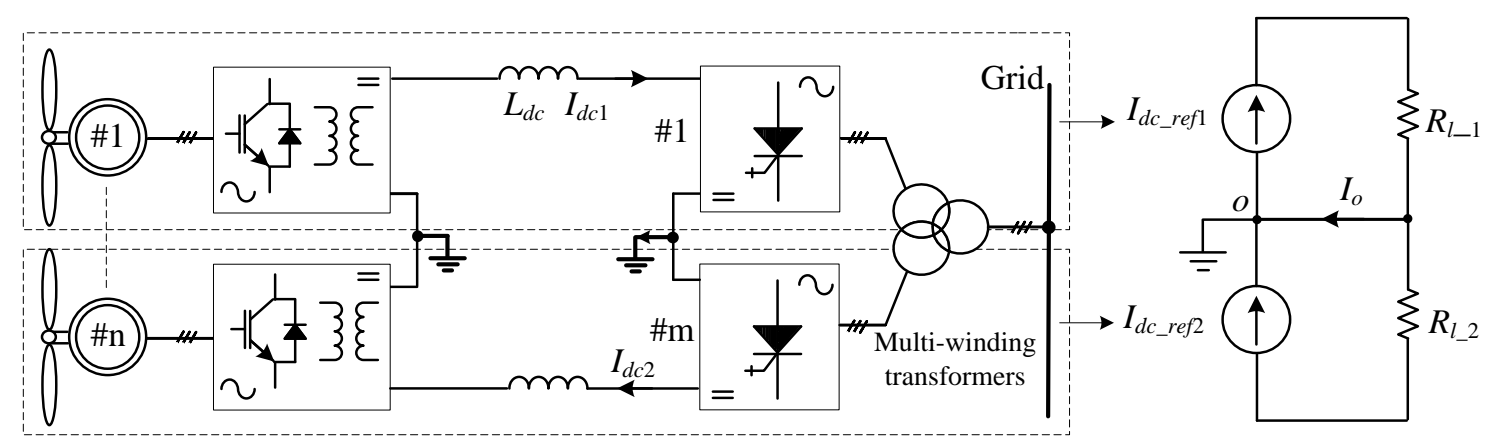

Figure 5-9 Bipolar offshore wind farm with the optimal dc-link current control.

For simplicity, the operation loss of onshore CSCs is taken as an example. As for a CSC, the switching $\left(P_{s w}\right)$ and conduction $\left(P_{c o n}\right)$ loss for CSCs can be expressed as follows based on the well-known method.

$$
\begin{gathered}
P_{s w}=f_{s w}\left(E_{o n}\left(I_{\text {nom }}, V_{\text {nom }}\right)+E_{\text {off }}\left(I_{\text {nom }}, V_{\text {nom }}\right)\right)\left(\frac{i}{I_{\text {nom }}}\right)\left(\frac{v}{V_{\text {nom }}}\right) \\
P_{\text {con }}=\left(v_{C E 0}+r \cdot i(t)\right) \cdot i(t)
\end{gathered}
$$

where $E_{\text {on }}$ and $E_{\text {off }}$ are given in the datasheet of the device, $f_{s w}$ is the switching frequency, $I_{n o m}$ and $V_{\text {nom }}$ are the rated current and voltage, while $i$ and $v$ are the instant values during the switching event, $v_{C E O}$ and $r$ are constant values used to calculate the on-state voltage of the device, and $i(t)$ is the current flowing through the device.

Given a fixed input power, the switching losses of the onshore CSCs for both schemes are same according to (5-17). This is in view of the fact that the voltage will accordingly increases as the current decreases. The conduction loss of the CSCs, on the other hand, is only related to its carrying current as shown in (5-18). Lower current gives lower conduction losses. Therefore, the conduction loss of the CSCs under the proposed scheme shown in Figure 5-9 will be greatly reduced compared with that under conventional scheme as shown in Figure 5-8.

For example, assuming the maximum wind speed in the upper part of the bipolar system is 1 $\mathrm{pu}$, while it is $0.9 \mathrm{pu}$ for the lower part. The resultant minimum dc-link reference currents are 1.1 $\mathrm{pu}\left(I_{d c_{-} r e f 1}\right)$ and $0.88 \mathrm{pu}\left(I_{d c_{-} r e f 2}\right)$ according to Figure 5-7. In the case of conventional control, the final dc-link current has to be set to $1.1 \mathrm{pu}$ and flows through both upper and lower parts. In the case of the proposed control, the upper and lower part are controlled independently with their 
own dc-link currents, $1.1 \mathrm{pu}$ for the upper part, and $0.88 \mathrm{pu}$ for the lower part. As previously mentioned, the switching loss of the lower part for the two schemes is same, while the conduction loss for the proposed scheme is reduced to $0.75 \mathrm{pu}$ according to (5-18) where the base conduction loss is the loss calculated under conventional scheme. As for an MV CSC, the conduction loss is weighting more than the switching loss with the typical relationship being around $P_{c o n}=2.4 P_{s w}[76]$. Therefore, the total operation loss of the onshore CSCs of the lower part based on the proposed scheme is reduced to $0.82 \mathrm{pu}$ compared with that based on conventional scheme. In addition, a larger mismatch of the maximum wind speed between upper and lower parts contributes to bigger operation loss savings. For example, the operation loss of the lower part will be reduced to $0.67 \mathrm{pu}$ when the maximum wind speeds for upper and lower parts are $1 \mathrm{pu}$ and $0.8 \mathrm{pu}$. The same principle also applies to the offshore converters, thus not repeated here.

Apart from achieving lower operation loss, the proposed dc-link current control gives the bipolar system higher flexibility. As mentioned earlier, the upper and lower parts of the system are controlled independently. Each part can operate on its own dc-link reference current as an independent system with the earth return. Therefore, one part can continue to transmit power in case the other one is out of service for whatever reason (power limitation mode or technical checking). In addition, a large transmission capacity can be achieved by extending the bipolar system to a multi-terminal system as shown in Figure 5-3.

In summary, the CS-based offshore wind farm with bipolar HVDC system features lower insulation requirement, giving lower cost, higher reliability, and higher power capacity. The optimal dc-link current control gives higher efficiency and flexibility.

\subsection{Performance investigation}

The performance of the bipolar operation of the CSC-based system and the optimal dc-link current control are verified by both simulation and lab-scaled experimental tests. Two CSCs are used in the following simulation and experiments. The parameters are listed in Appendix.

\subsubsection{Simulation investigation}

Figure 5-10 illustrates the simulated performance of the proposed bipolar system under stepped dc-link currents. $i_{g 1 \_a b c}$ and $i_{g 2 \_a b c}$ represent three-phase load currents for Module \#1 and \#2; $I_{d c 1}$ 
and $I_{d c 2}$ are the positive and negative dc-link currents of the bipolar system, respectively; $I_{o}$ is the current through the earth return. Before $t=2 \mathrm{~s}$, Module \#1 and \#2 are operating under steady state with the dc-link currents being around $I_{d c 1}=0.6 \mathrm{pu}, I_{d c 2}=0.44 \mathrm{pu}$. At $t=2 \mathrm{~s}$, the dc-link reference current for Module \#1 is stepped up to $1 \mathrm{pu}$, while Module \# 2 is increasing from 0.44 pu to $0.8 \mathrm{pu}$. During this period, the dc-link currents are well tracking their references as shown in Figure 5-10. The current through the earth return $I_{o}$ is accordingly changed. This process continues until the system are operating under steady state again.

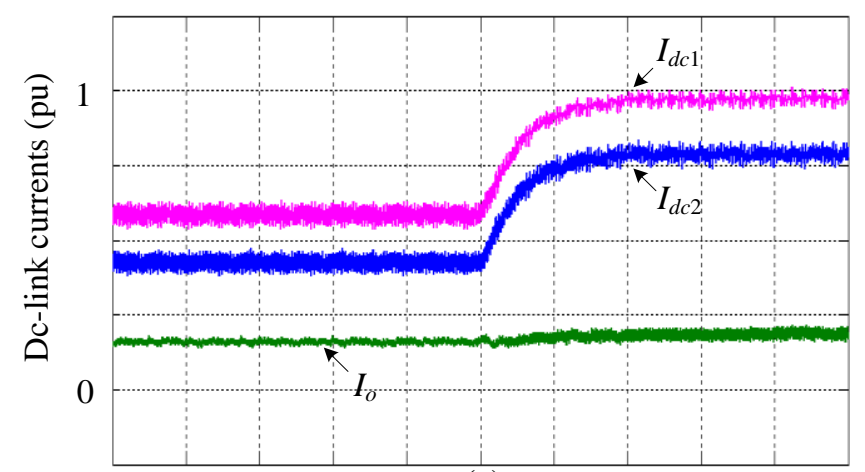

(a)

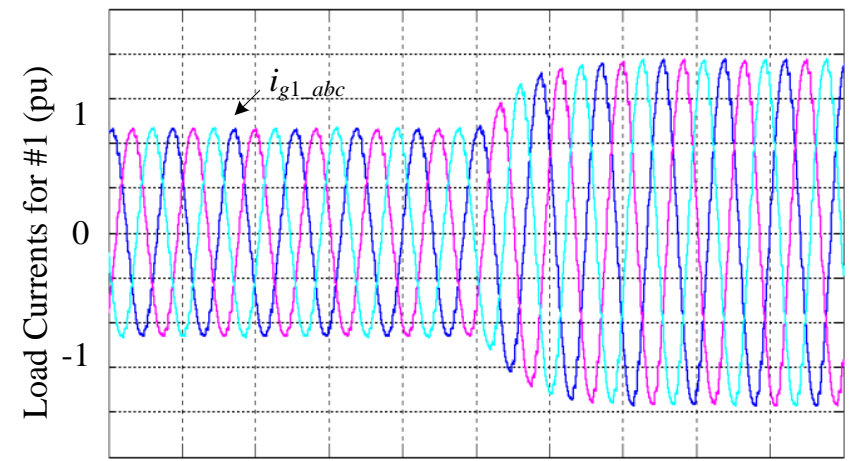

(b)

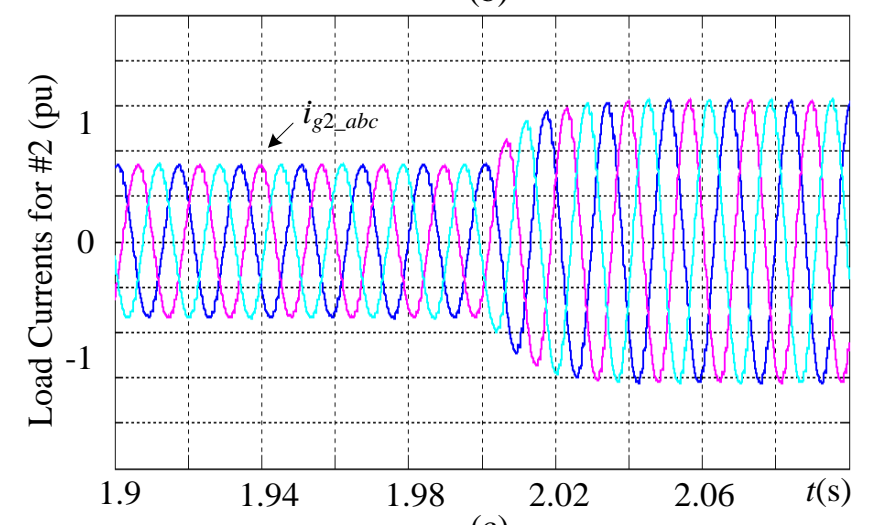

(c)

Figure 5-10 Simulated performance of the bipolar system under stepped dc-link current. 
Figure 5-11 illustrates the simulated performance of the proposed bipolar system when one module is bypassed. Before $t=2 \mathrm{~s}$, Module \#1 and \#2 are operating under rated condition with $I_{d c 1}=I_{d c 2}=1 \mathrm{pu}$. At $t=2 \mathrm{~s}$, the dc-link reference current for Module \#1 is set to 0 pu purposely. As a result, the load current for Module \#1 and its dc-link current are decreasing to $0 \mathrm{pu}$, while Module \# 2 is operating at 1 pu without any disruption.

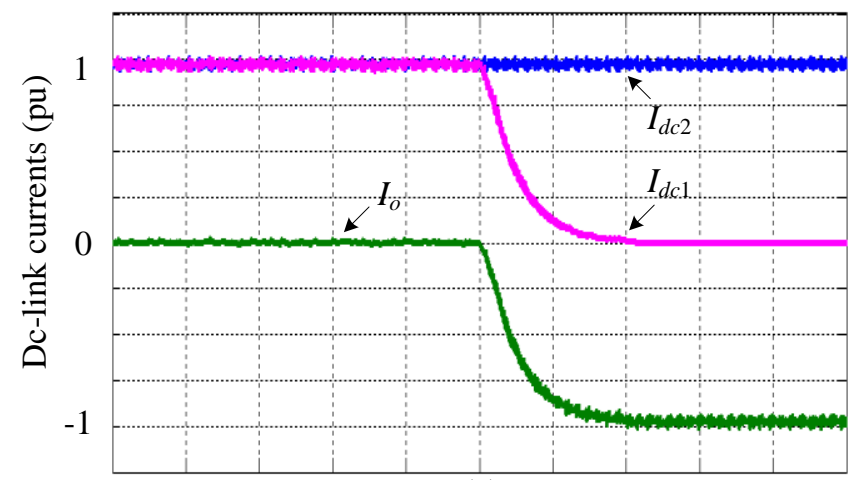

(a)

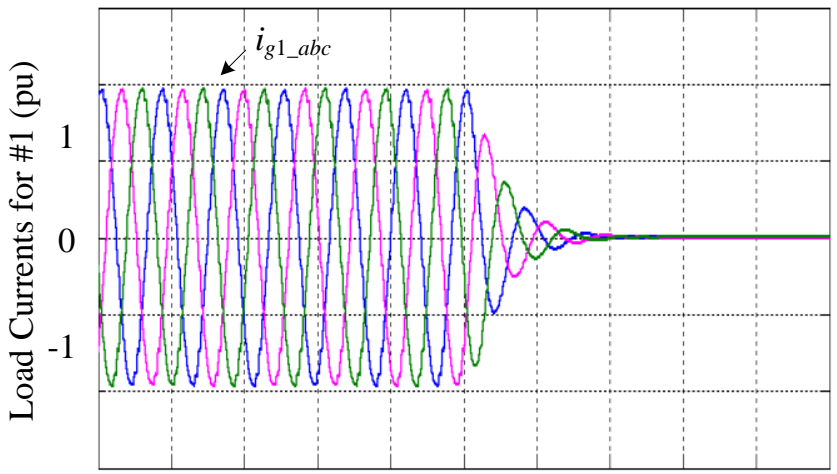

(b)

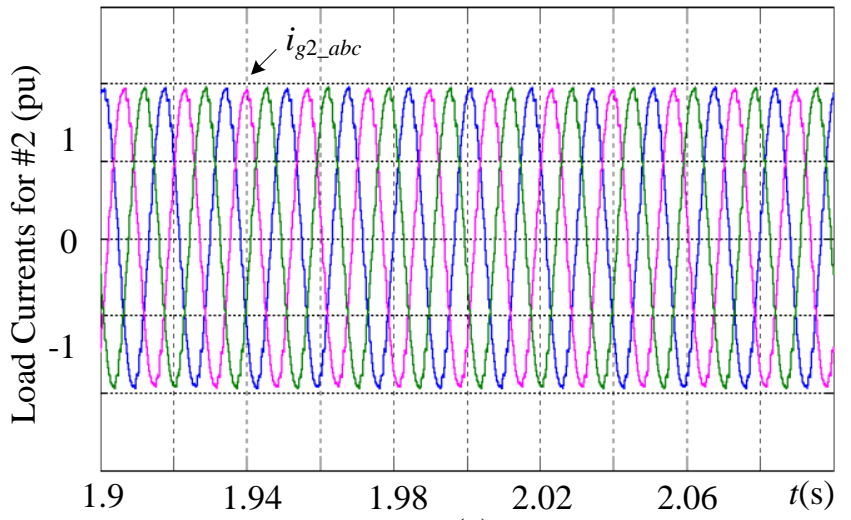

(c)

Figure 5-11 Simulated performance of the bipolar system when one module is bypassed. 


\subsubsection{Experimental verification}

Figure 5-12 shows the experimental performance of the bipolar system under stepped dc-link currents. Figure 5-12 (a) shows the dc-link currents and the neutral current. $I_{d c 1}$; (b) load currents of Module \#1. $i_{g 1 \_a b c}$; and (c) load currents of Module \#2. $i_{g 2 \_a b c}$. Before time $t$ Module \#1 and \#2 are operating under steady state with the dc-link currents being around $I_{d c 1}=0.6 \mathrm{pu}(3 \mathrm{~A}), I_{d c 2}=$ $0.44 \mathrm{pu}(2.2 \mathrm{~A})$. At time $t$, the dc-link reference current for Module \#1 is set to $1 \mathrm{pu}(5 \mathrm{~A})$, while Module \# 2 is set to $0.8 \mathrm{pu}(4 \mathrm{~A})$. Accordingly, $I_{d c 1}$ and $I_{d c 2}$ are tracking their references as shown in Figure 5-12. The current through the earth return $I_{o}$ is changed accordingly.

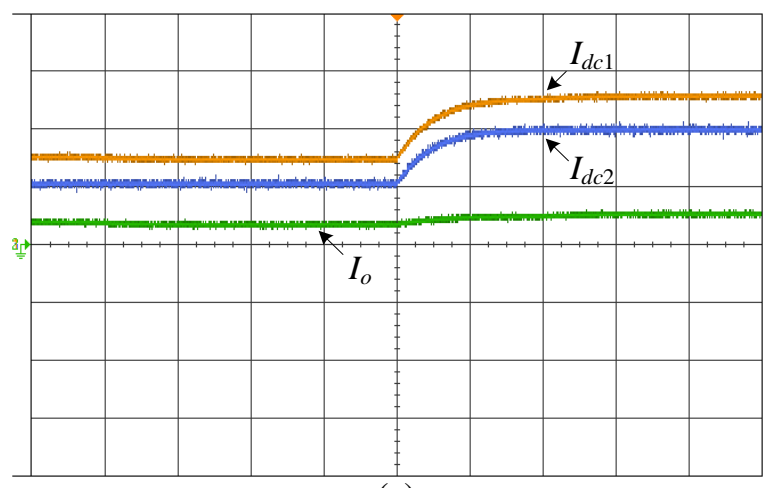

(a)

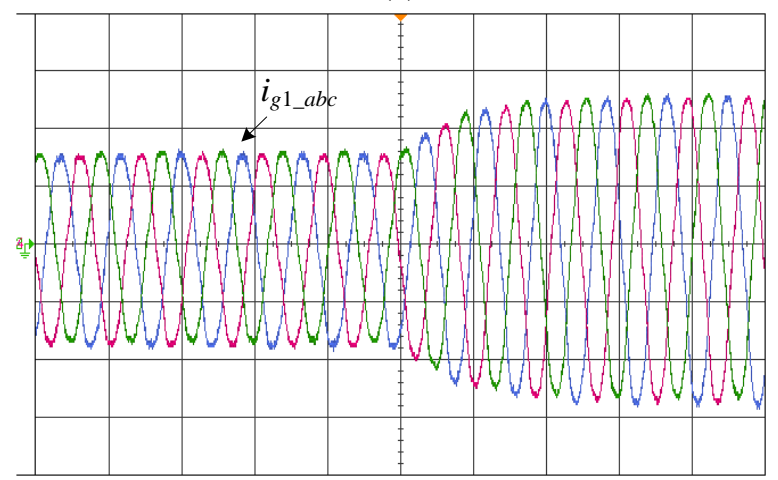

(b)

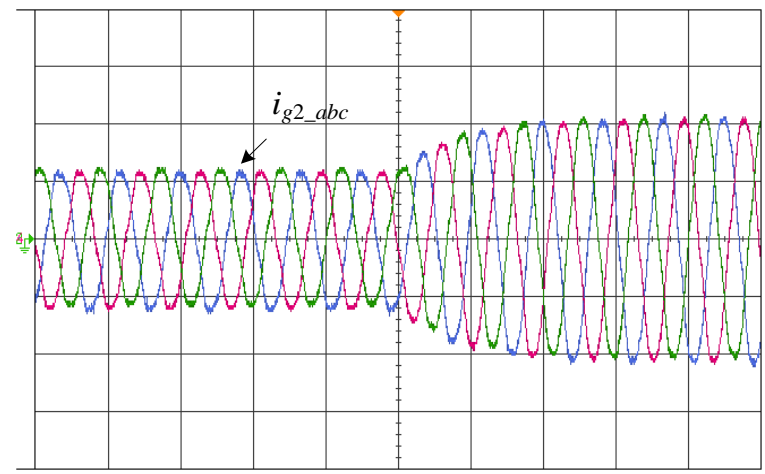

(c)

Figure 5-12 Experimental performance of the bipolar system under stepped dc-link currents. 
Figure 5-13 illustrates the experimental performance of the bipolar system when one module is bypassed. All the conditions are same with those in simulation. The two modules can be operating independently with the help of separate dc-link current control.

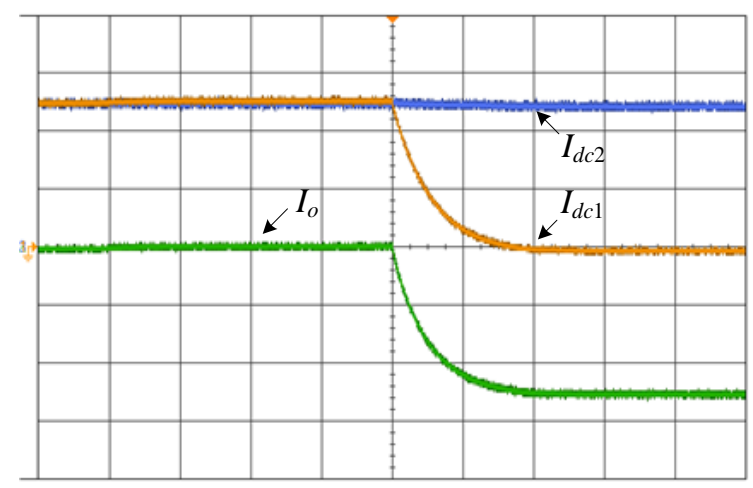

(a)

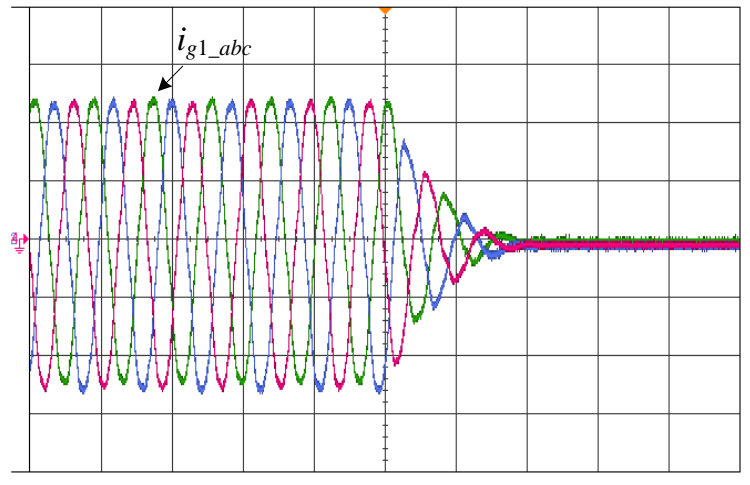

(b)

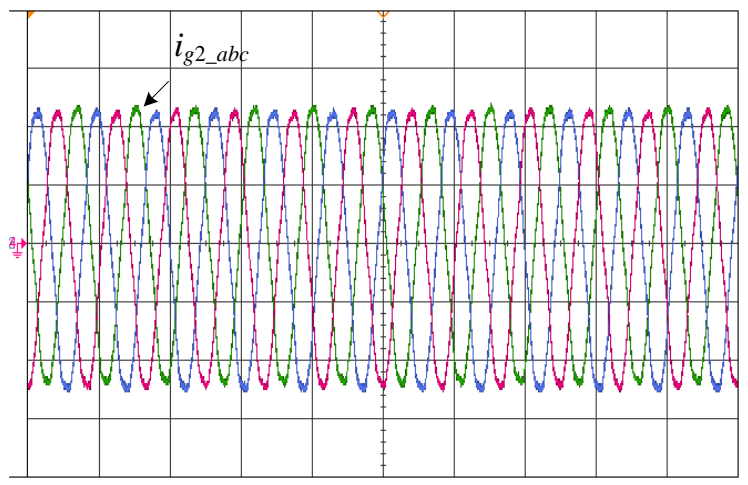

(c)

Figure 5-13 Experimental performance of the bipolar system when one module is bypassed.

\subsection{Conclusions}

In this chapter, the bipolar operation of the CSC-based series-connected offshore wind farm is investigated. Compared with traditional monopolar operation mode, bipolar mode gives lower insulation level, thus, contributing to lower cost and higher reliability. Besides, the bipolar 
system can be extended to a multi-terminal system with larger amount of power transferring, while the capacity of a monopolar system is limited to a certain number mainly due to the constraint of the increased insulation requirement.

An optimal dc-link current control is proposed for the bipolar system. The equivalent circuit of the bipolar system is derived. The minimum dc-link current of the wind farm is derived and defined, based on which an optimal dc-link current control with higher efficiency and flexibility is proposed.

Both simulations and experiments are carried out to verify the performance of the bipolar system and the optimal dc-link current control scheme. 


\section{Chapter 6}

\section{Optimized Control Strategy for Offshore}

\section{Wind Farm with Reduced Cost and}

\section{Improved Efficiency}

As mentioned in the previous chapters, the proposed CSC-based series-connected offshore wind farm features smaller size and weight and higher reliability and flexibility. However, another two issues exist. One is the high cost of grid-side CSCs. The nominal number of onshore CSCs is conventionally set to be same as that of the offshore turbine-generator units. This leads to higher cost on power converters. The other is the reduced efficiency due to the inconsistency of wind speed at each turbine. In practice, the wind speed at each turbine cannot be guaranteed to be identical. The inconsistency of wind speed generates different dc-link current references, among which the maximum one is selected to be the final dc-link current of the wind farm. This leads to a reduced overall efficiency. Therefore, the objective of this chapter is to propose an optimized strategy for the CSC-based offshore wind farm with reduced cost and improved efficiency.

This chapter starts with a brief introduction of conventional strategy for the CSC-based offshore wind farm, followed by the proposed strategy. The nominal number of onshore seriesconnected CSCs is optimized that lower cost and higher efficiency can be obtained under rated condition. What is more, a bypass operation is introduced to grid-side CSCs, based on which the efficiency of the wind farm is improved under derating conditions. Both simulation and experimental results are provided.

\subsection{Conventional control strategy}

In practice, the wind speed at each turbine cannot be guaranteed to be identical as shown in Figure 6-1. The inconsistency of wind speed generates different dc-link reference currents for each turbine unit. However, due to the series connection, the dc-link current through all the 
converters is inevitably the same. This therefore addresses a considerable challenge for the system operation. To overcome the above challenge, a coordinated control is proposed in [40].

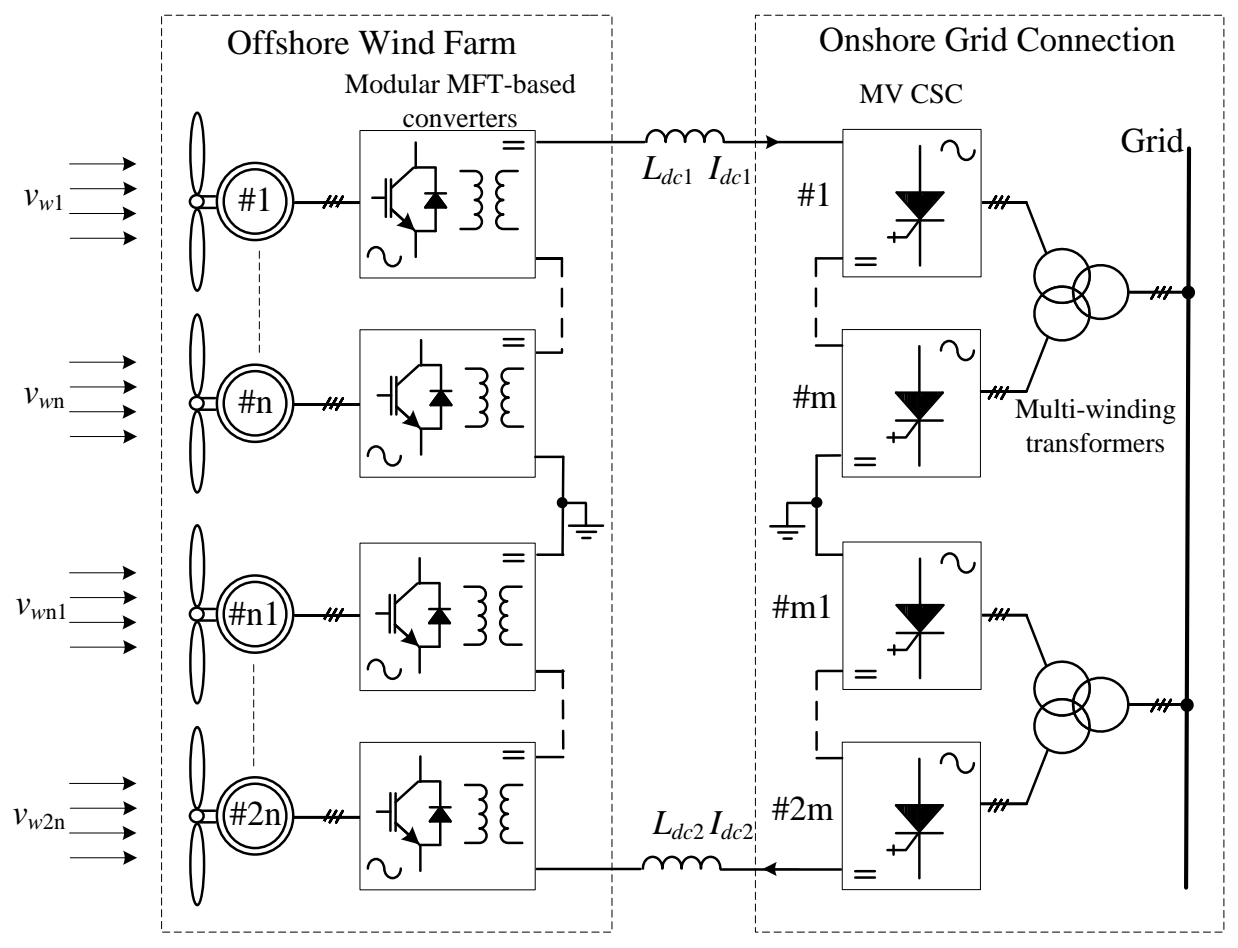

Figure 6-1 CSC-based offshore wind farm with different wind speeds.

In the coordinated scheme [40], the nominal number of grid-side series-connected CSCs is set to be same as that of offshore turbine-generator units, that is $\mathrm{m}=\mathrm{n}$ as shown in Figure 6-1. Second, the maximum dc-link current reference among all generated reference currents is selected to be the final dc-link reference current of the wind farm to ensure both offshore and onshore control objectives. Third, all the grid-side CSCs are involved during operation.

However, there are two issues associated with the coordinated scheme. One is the high cost of grid-side CSCs. The other is the lower efficiency of the wind farm caused by the inconsistent wind speed at each turbine. Therefore, the main objective of this chapter is to propose an optimized strategy for the wind farm with lower cost and higher efficiency.

\subsection{Optimized control strategy}

As mentioned earlier, conventional strategy achieves all the control objectives, but at the expense of high cost and lower efficiency on grid-side CSCs. Therefore, in this chapter, an 
optimized control strategy is proposed [77]. It aims at achieving lower cost and higher efficiency with all the control objectives being ensured simultaneously.

\subsubsection{Optimized nominal number of CSCs with lower cost and higher efficiency}

As presented previously, a proper "minimum" dc-link current is required to ensure all the control objectives and achieve high efficiency. The dc-link currents determined by generatorand grid-side converters under UPF and MPPT in the full operation range are illustrated in Figure 6-2. Note the base dc-link current is the minimum current determined by the generatorside converter. The base power is the maximum captured power under rated wind speed.

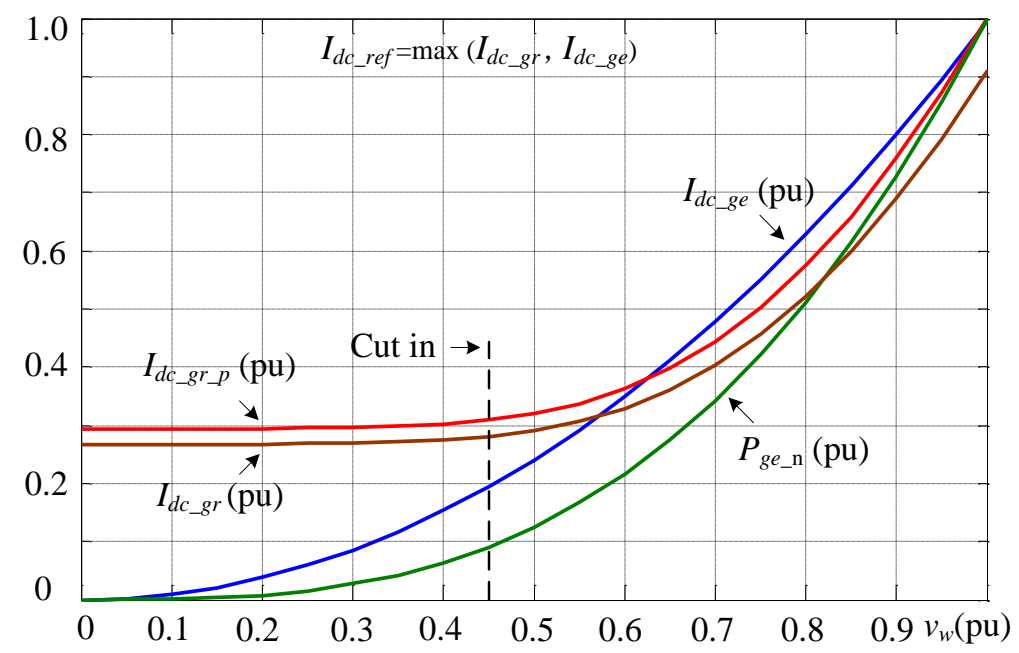

Figure 6-2 Final dc-link reference current determination.

As mentioned earlier, the final dc-link reference current should be equal or higher than the larger one between the generator- and grid-side reference currents, that is

$$
I_{d c_{-} r e f}=\max \left(I_{d c_{-} g e}, I_{d c_{-} g r}\right)
$$

Figure 6-2 shows that the generator-side reference current determines the final dc-link reference current of the wind farm in high wind speeds ranging from around $0.57 \mathrm{pu}$ to $1 \mathrm{pu}$, while it is the grid-side one under low speeds.

In conventional scheme, the number of onshore CSCs (m) is set to be same as that of offshore turbine-generator units (n). This introduces a high cost. In the case of a single turbine unit shown in Figure 6-2, the final minimum dc-link reference current is determined by the generator-side 
converter, that is $1 \mathrm{pu}$, while it is $0.9 \mathrm{pu}$ for the grid-side converter. As a result, the nominal modulation index for the grid-side CSC is 0.9. This also applies to all the onshore CSCs as the nominal power is evenly distributed among them and $\mathrm{m}=\mathrm{n}$. This therefore results in a derating operation for CSCs, leading to additional cost on CSCs as more CSCs are required to transfer the nominal power.

Therefore, less CSCs will be needed when operating the onshore CSCs under $m_{a}=1$ and maintaining the dc-link reference current of the wind farm. First, the nominal dc-link current of the wind farm $\left(I_{d c_{-} \text {rated }}\right)$ is calculated based on the nominal power $P_{W F_{-} \text {rated }}$ according to (6-1). Second, the nominal power of the wind farm is evenly distributed among an optimized number $(m)$ of onshore CSCs which are operating under $m_{a}=1$. The resultant nominal power for each onshore CSC is $P_{W F_{-} \text {rated }} / \mathrm{m}$. Then, the optimized number of onshore CSCs, that is $m$, is derived as follows. Note that the solutions of (6-2) may be non-natural numbers that its rounding natural number which is equal or larger than the solution of (6-2) should be selected.

$$
m=\frac{2}{3} \frac{P_{W F_{\text {_rated }}}}{V_{\text {sd }}} \cdot \frac{\left(1-\omega_{g}{ }^{2} L_{g} C_{f}\right)}{\sqrt{I_{d c_{-} \text {rated }}{ }^{2}-\left(\omega_{g} C_{f} V_{s d}\right)^{2}}}
$$

For example, assuming a wind farm having 10 turbine units. According to (10), only 9 CSC are needed when using the proposed scheme which gives a $10 \%$ reduction in cost.

In addition, the 9-CSC system gives a higher efficiency compared with the 10-CSC system. The operation losses of the onshore CSCs include switching $\left(P_{s w}\right)$ and conduction $\left(P_{c o n}\right)$ losses shown below.

$$
\begin{gathered}
P_{s w}=f_{s w}\left(E_{o n}\left(I_{n o m}, V_{n o m}\right)+E_{o f f}\left(I_{n o m}, V_{n o m}\right)\right)\left(\frac{i}{I_{n o m}}\right)\left(\frac{v}{V_{n o m}}\right) \\
P_{c o n}=\frac{1}{T} \int_{0}^{T} v_{C E}(t) \cdot i(t) \cdot f(t) d t
\end{gathered}
$$

where $E_{\text {on }}$ and $E_{\text {off }}$ are given in the datasheet of the device, $f_{s w}$ is the switching frequency, $I_{n o m}$ and $V_{\text {nom }}$ are the rated current and voltage, while $i$ and $v$ are the instant values during the switching event, $v_{C E}(t)$ is the on-state voltage according to output characteristic $i(t)=f\left(v_{C E}(t)\right)$, $i(t)$ is the current flowing through the device, and $f(t)$ is a function of pulse pattern with the switch turn-on $=1$ and switch turn-off $=0$. 
As for a CSC, the switching scheme is: only two switches in the converter are conducted at any instant with one in the top half of the CSC bridge and the other in the bottom half [22]. Hence, a three-phase CSC can be equivalent to a one-leg converter for loss evaluation shown in Figure 6-3.

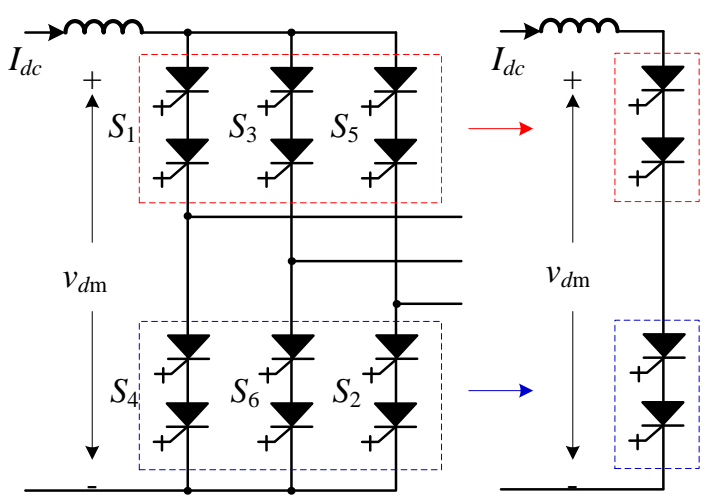

Figure 6-3 Equivalent circuit of a CSC used for loss evaluation.

Then the conduction losses for one CSC can be simplified as:

$$
P_{c o n}=2 v_{C E}(t) \cdot i(t)
$$

Equation (6-5) tells that the 9-CSC system has a $10 \%$ reduction in conduction loss compared with the 10-CSC system as they share the same dc-link current. Besides, in an MV CSC where SGCT is employed, the conduction loss is around 2.5 times of the switching loss [76]. Therefore, about $8 \%$ of total loss of onshore CSCs under a rated condition can be saved.

The switching losses for the 10-CSC and 9-CSC system are

$$
\begin{aligned}
& P_{s w_{-} 10}=6 f_{s w}\left(E_{o n}\left(I_{n o m}, V_{n o m}\right)+E_{o f f}\left(I_{n o m}, V_{n o m}\right)\right)\left(\frac{I_{d c}}{I_{n o m}}\right)\left(\frac{v_{1}+v_{2}+\cdots+v_{10}}{V_{n o m}}\right) \\
& P_{s w_{-} 9}=6 f_{s w}\left(E_{o n}\left(I_{n o m}, V_{n o m}\right)+E_{o f f}\left(I_{n o m}, V_{n o m}\right)\right)\left(\frac{I_{d c}}{I_{n o m}}\right)\left(\frac{v_{1}^{\prime}+v_{2}^{\prime}+\cdots+v_{9}^{\prime}}{V_{n o m}}\right)
\end{aligned}
$$

where $v_{1}, \cdots, v_{10}$ is the average dc voltage across one CSC for the 10-CSC system, while $v_{1}^{\prime}, \cdots, v_{9}^{\prime}$ is for the 9-CSC system.

Obviously, the 9-CSC and 10-CSC system are with same switching losses as they share same dc-link current and same dc-link voltage.

It is worth noting that the de-rating voltage/power in conventional scheme, does not benefit each CSC in terms of cost of the switching devices. Instead, the switches (voltage rating and 
number per phase) of each CSC for both conventional and the proposed schemes are same which is unique for MV CSCs. The switches used in MV CSC are SGCT. Only one type of commercial SGCT exists in the market (6500 V SGCT designed by ABB). The numbers of used switches (SGCT) per phase in commercial MV drives (by Rockwell Automation Canada) are 2 for $2400 \mathrm{~V}$

drives, 4 for $3300 \mathrm{~V} / 4160 \mathrm{~V}$ drives, and 6 for $6600 \mathrm{~V}$ drives [70]. Therefore, the cost of each CSC for both schemes are same, while the proposed one needs less CSCs, thus lowering the cost on CSCs. In addition, the proposed scheme also features higher efficiency, thus benefiting lower cost to the thermal and cooling system of onshore CSCs.

According to (5-14), the resultant grid-side dc-link reference current $I_{d c_{-} g r_{-} p}$ with the optimized number is derived as

$$
I_{d c_{-} g r_{-} p}=\sqrt{\left\{\left(1-\omega_{g}{ }^{2} L_{g} C_{f}\right)\left(\frac{2}{3} \frac{P_{W F}}{m V_{s d}}\right)\right\}^{2}+\left(\omega_{g} C_{f} V_{s d}\right)^{2}}
$$

Figure 6-2 shows that the final dc-link currents of the wind farm for both schemes are same in a major operation range from around $0.6 \mathrm{pu}$ to $1 \mathrm{pu}$, while the proposed scheme generates a slightly higher reference current under low wind speeds ranging from a cut-in speed $(0.45 \mathrm{pu})$ to around $0.6 \mathrm{pu}$. Note that the slightly higher dc-link current in low wind speeds produces slightly higher losses, but does not matter so much as the generated power of the wind farm is greatly reduced in low wind speeds as shown in Figure 6-2. In summary, all the control objectives can be ensured as the final dc-link current of the system is maintained.

In summary, the optimized nominal number of onshore CSCs gives a $10 \%$ reduction in cost and an $8 \%$ in loss under a rated condition.

\subsubsection{Optimized control of CSCs with higher efficiency}

As mentioned earlier, the inconsistency of wind speed at each turbine leads to a reduced efficiency. Assuming the wind speed at each turbine is identical, that is $1 \mathrm{pu}$, for example, the generated power is $1 \mathrm{pu}$ as well as its dc-link reference current (1 pu) as shown in Figure 6-2. The total power of the wind farm is evenly distributed among all the onshore CSCs. Then the resultant grid-side determined dc-link reference current is 1 pu. Finally, the dc-link reference current $I_{d c_{-} r e f}$ of the wind farm is set to 1 pu to ensure all control objectives. 
However, it is not the case in practice as the wind speed at each turbine cannot be guaranteed to be identical. For example, when the maximum wind speed among all is $1 \mathrm{pu}$ and the others are lower, the available power of the wind farm $P_{W F}$ will be greatly reduced and so does the power of each onshore CSC compared with the previous case where all the speeds at turbines are $1 \mathrm{pu}$. The resultant grid-side determined dc-link reference current is therefore reduced to be less than 1 pu. The final dc-link reference current of the wind farm, however, should be set to be the maximum one between generator- and grid-side ones, that is $1 \mathrm{pu}$, to ensure all the control objectives. Then, the operating modulation index for each CSC is less than 1. As analyzed earlier, a smaller modulation index requires more CSCs to transfer a given power, thus leading to higher operation losses.

To lower the loss of onshore CSCs under the condition of inconsistent wind speeds, a bypass operation is introduced to onshore CSCs. First. an optimized number of CSCs instead of all is operating while the others are bypassed according to the captured wind power. The number of operating CSCs is optimized by enabling the involved CSCs to operate under $m_{a}=1$ and keeping the current dc-link current of the wind farm unchanged. The former is to lower operation loss, while the latter is to ensure all the control objectives. Second, when the captured wind power of the wind farm increases, some or all of the bypassed CSCs are shifted to normal operation again. The optimized number of CSCs $(l)$ is derived based on (6-7):

$$
l=\frac{\left(1-\omega_{g}{ }^{2} L_{g} C_{f}\right)\left(\frac{2}{3} \frac{P_{W F}}{V_{s d}}\right)}{\sqrt{I_{d c_{-} r e f}{ }^{2}-\left(\omega_{g} C_{f} V_{s d}\right)^{2}}}
$$

The bypass operation of a CSC can be achieved by (1) turning on one-leg switches of the converter, or (2) turning on all three-leg switches of the converter and disconnecting it from the grid, or (3) turning on one-leg switches of the converter and additional bypass switch/parallelconnected switches (GTO, for example) across the input terminals of the CSC.

A comparison of operation loss between conventional and the proposed schemes for a 10turbine system is shown in Table 6-1. As mentioned previously, only 9 CSCs are needed to be compatible with the 10-turbine system. All values except the number " $m$ " and " $l$ " are based on the per-unit system. The based power and base current are defined as same as previously defined. The base switching $\left(P_{s w}\right)$ and conduction $\left(P_{c o n}\right)$ loss are those generated by one CSC based on 
conventional scheme. A mismatch within $20 \%$ is purposely set for the wind speeds of the 10 turbine system as shown in Table 6-1. The wind speed at turbine \#1 and 2 is 1 pu, while it is 0.9 pu for unit \#3, 4, 5, and 0.8 pu for \#6, 7, 8, 9, and 10, respectively. The resultant generated power is $1 \mathrm{pu}, 0.729 \mathrm{pu}$, and $0.512 \mathrm{pu}$ with the total available power of the 10 -turbine system $P_{W F}$ being $6.964 \mathrm{pu}$. The final dc-link reference currents for both schemes are same at $1 \mathrm{pu}$ so that all the control objectives achieved by conventional scheme can be completely guaranteed by the proposed one. However, all the 9 CSCs are required for conventional scheme, while the proposed one only needs 6 CSCs based on (6-8).

Table 6-1 Comparison Between Conventional and Proposed Schemes

\begin{tabular}{|c|c|c|c|c|c|c|c|c|c|c|}
\hline Items (pu) & \#1 & $\# 2$ & $\# 3$ & \#4 & $\# 5$ & \#6 & \#7 & \#8 & \#9 & $\# 10$ \\
\hline Wind speed $\left(v_{w}\right)$ & 1 & 1 & 0.9 & 0.9 & 0.9 & 0.8 & 0.8 & 0.8 & 0.8 & 0.8 \\
\hline Generated power $\left(P_{g e \_n}\right)$ & 1 & 1 & 0.729 & 0.729 & 0.729 & 0.512 & 0.512 & 0.512 & 0.512 & 0.512 \\
\hline Total power $\left(P_{W F}\right)$ & \multicolumn{10}{|c|}{6.747} \\
\hline $\begin{array}{l}\text { Generator-side reference current } \\
\left(I_{d c_{-} \text {gen }}\right)\end{array}$ & 1 & 1 & 0.8 & 0.8 & 0.8 & 0.8 & 0.63 & 0.63 & 0.63 & 0.63 \\
\hline \multicolumn{11}{|c|}{ Conventional Scheme } \\
\hline $\begin{array}{l}\text { Grid-side reference current } \\
\left(I_{d c_{-} g}\right)\end{array}$ & \multicolumn{10}{|c|}{0.7} \\
\hline $\begin{array}{l}\text { Final dc-link reference current } \\
\left(I_{d c_{\_} r e f}\right)\end{array}$ & \multicolumn{10}{|c|}{1} \\
\hline Number of involved CSCs $(l)$ & \multicolumn{10}{|c|}{9} \\
\hline \multicolumn{11}{|c|}{ Proposed Scheme } \\
\hline $\begin{array}{l}\text { Grid-side reference current } \\
\left(I_{d c_{-} g r_{-}}\right)\end{array}$ & \multicolumn{10}{|c|}{1} \\
\hline $\begin{array}{l}\text { Final dc-link reference current } \\
\left(I_{\left.d c_{\text {rref }}\right)}\right.\end{array}$ & \multicolumn{10}{|c|}{1} \\
\hline Number of involved CSCs $(l)$ & \multicolumn{10}{|c|}{6} \\
\hline
\end{tabular}

Besides, different combinations of wind speeds of the 10-turbine system have different bypass solutions. For example, 4 CSCs will be bypassed when more offshore units are operating under a wind speed of $0.8 \mathrm{pu}$. In addition, a larger mismatch among wind speeds leads to more CSCs being bypassed. Theoretically, the maximum number of bypassed CSCs is 7 which occurs when one turbine is operating under a rated wind speed, while the others are all under a very low wind speed. Note that the above 10-turbine system can be considered a per-unit system and can be extended to a N-turbine system.

The operation loss of the onshore CSCs before and after bypass operation can be obtained with the same manner as mentioned in last chapter. First, the switching loss of the onshore CSCs 
before and after bypass operation is same, which can be verified by the same principle in the previous section, thus not repeated here. Second, the conduction loss of the operating CSCs before and after bypass operation is same as the dc-link current is kept same. The bypassed CSCs, on the other hand, give possibly different loss savings depending on their bypass methods: (1) when turning on one-leg switches, the CSC is bypassed. Under such a condition, the conduction loss before and after bypass operation is same; (2) when turning all switches and disconnecting the CSC from the grid, the CSC is bypassed. Under such a condition, each leg carries one third of the dc-link current. Accordingly, the on-state voltage $v_{C E}(t)$ of the involved SGCTs, is decreased with a factor of around 27\% [78], giving a reduction of $27 \%$ in conduction loss. Due to different weighting factors between switching and conduction losses for an MV CSC $\left(P_{c o n}=2.4 P_{s w}\right)$ [76], the finalized operation loss of each bypassed CSC is reduced with a factor of around $20 \%$. However, this bypass method requires additional switches to disconnect the CSC from the grid and these switches introduce losses during normal operation; and (3) when turning on one-leg switches and the additional bypass switch/switches connected across the input terminals of the CSC, the CSC is bypassed. Under such a condition, the conduction loss is reduced which is similar to the case of (2). No extra loss is introduced by the bypass switch/switches during normal operation. The cost for the bypass switch/switches is acceptable as low-cost GTO can be used. Note that, an optimal design for such an offshore wind farm should consider all the factors simultaneously, such as cost, efficiency, reliability, and so on. Here, the "optimized scheme", however, does not consider them simultaneously. Thus, a further study is needed when implementing the scheme in practice.

To sum up, the series-connected CSC-based system with the help of the proposed optimized strategy achieves a lower cost and a higher efficiency with all the control objectives being ensured simultaneously. The main conclusions are summarized in Table 6-2.

Table 6-2 Comparisons Between Conventional and Proposed Strategies

\begin{tabular}{|l|l|l|l|}
\hline \multicolumn{2}{|c|}{ Items } & \multicolumn{1}{c|}{ Conventional } & \multicolumn{1}{c|}{ Proposed } \\
\hline $\begin{array}{l}\text { Rated Condition } \\
\text { (all wind speeds at each } \\
\text { turbine are under rated } \\
\begin{array}{l}\text { condition without } \\
\text { inconsistency) }\end{array}\end{array}$ & $\begin{array}{l}\text { Nominal number of } \\
\text { onshore CSCs needed } \\
(m)\end{array}$ & $\begin{array}{l}\mathrm{l}=n \\
(n \text { : number of } \\
\text { offshore turbine } \\
\text { units })\end{array}$ & $(m=0.9 n)(6-2)$ \\
\cline { 2 - 4 } & Cost of onshore CSCs & $1 \mathrm{pu}$ & $0.9 \mathrm{pu}$ \\
\cline { 2 - 4 } & Loss of onshore CSCs & $1 \mathrm{pu}$ & $0.92 \mathrm{pu}$ \\
\hline Derating Condition & Number of operating & All & A part of CSCs \\
\hline
\end{tabular}




\begin{tabular}{|l|l|l|l|}
\hline $\begin{array}{l}\text { (inconsistency of wind } \\
\text { speed at each turbine) }\end{array}$ & onshore CSCs $(l)$ & $(l=\mathrm{n})$ & $(l<m<n)(6-8)$ \\
\cline { 2 - 5 } & Loss of onshore CSCs & $1 \mathrm{pu}$ & $\begin{array}{l}\text { depending on the maximum } \\
\text { mismatch and different } \\
\text { combinations of wind speeds } \\
\text { and the bypass methods }\end{array}$ \\
\hline All the control objectives of a wind farm are ensured & Yes & Yes \\
\hline
\end{tabular}

\subsubsection{Overall strategy of the wind farm}

The overall control strategy of the proposed CSC-based series-connected offshore wind farm is summarized as shown in Figure 6-4. It consists of three parts: wind farm supervisory control (WFSC), offshore converters control, and onshore converters control.

The WFSC acts as a single centralized unit, which has inputs from system operator orders, available power from each turbine-generator unit, monitoring signals from each turbinegenerator and grid-side converter unit, and measured grid voltage at the wind farm grid connection. The WFSC outputs active power to each turbine-generator unit, and dc-link current reference and reactive power reference to grid-side converters. The WFSC operates the wind farm either in maximum power mode or power limitation mode according to the system operator's demands. In maximum power mode, every wind turbine in the wind farm is allowed to operate in MPPT control mode to capture the maximum wind power.

The control objectives of offshore converters include independent MPPT and voltage/current balance control for MV/LV turbine systems. First, the primary objective for offshore converters is to capture the maximum wind power from varying wind speeds. This can be achieved by regulating the modular MFT-based converter as shown in Figure 6-4. By controlling each turbine converter independently, all turbines are able to achieve MPPT irrespective of the inconsistency of wind speed at each turbine. Second, an additional voltage balance control is required for MV configuration and an average current balance control for LV configuration. The constituent modules of the modular MFT-based converter in both MV and LV configurations are designed to be identical. However, they cannot be guaranteed to be identical in practice leading voltage/current imbalance among the constituent modules. Thus, additional control scheme is needed: a voltage balancing control for MV configuration, and a current balancing scheme for LV configuration. In summary, both MPPT and voltage/current balancing are ensured by applying the control scheme shown in Figure 6-4. 
The control objectives of onshore converters are dc-link current control, reactive power control, voltage balancing control, and the optimized control scheme for series-connected CSCs. First, the performance of the wind farm with bipolar HVDC system is investigated. Compared with monopolar mode, bipolar mode features lower insulation level, thus contributing to lower cost and higher reliability, but with a technical challenge for its dc-link current control. Accordingly, an optimal dc-link current control with higher efficiency and flexibility is proposed.

Second, a combined control scheme is proposed for series-connected CSCs. In the CSC-based offshore wind farm configuration, a number of MV CSCs are connected in series to form a centralized dc/ac inverter to transfer the captured wind power to the grid system. In practice, the tolerance in component leads to tolerance in the grid-side power factor and the power/input voltage among series-connected CSCs. To ensure both grid-side power factor and power balancing among CSCs, a combined control scheme is proposed as shown in Figure 6-4.

Third, an optimized control strategy is proposed for the offshore wind farm with lower cost and higher efficiency. The nominal number of onshore CSCs is optimized that a lower cost is obtained. An optimized control is proposed for onshore CSCs that the overall efficiency of the wind farm under the inconsistency of wind speeds is improved. In addition, the scheme is implemented without any additional components or auxiliary circuits.

In summary, the proposed modular MFT-based configuration features smaller size and weight which is preferred for offshore applications. Besides, lower cost, higher efficiency, and higher reliability and flexibility are obtained with the help of the proposed control schemes summarized in Figure 6-4. 

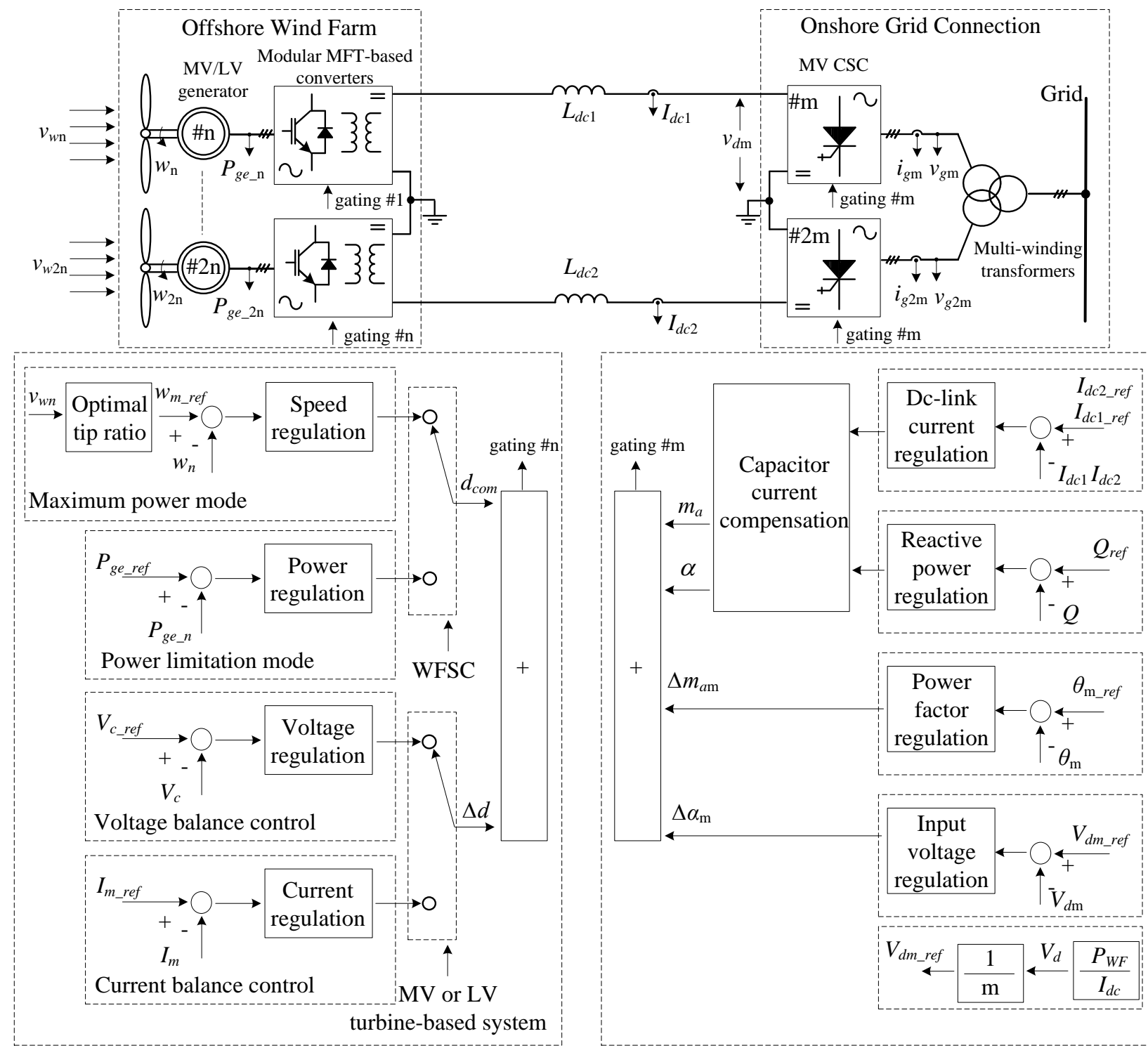

Offshore control

Onshore control

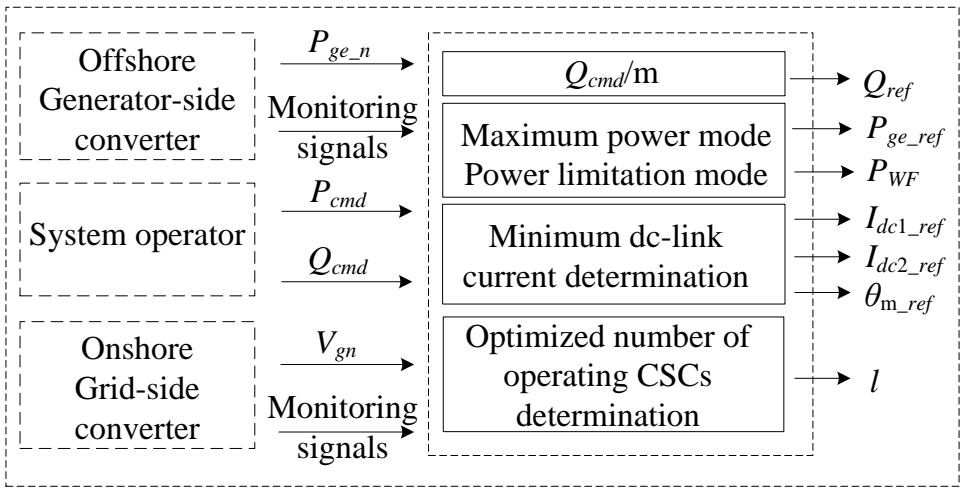

Wind farm supervisory control

Figure 6-4 Overall control strategy of the CSC-based offshore wind farm. 


\subsection{Performance investigation}

As shown in Figure 6-4, the overall control strategy of the wind farm consists of a couple of parts. The present focus is the optimized strategy of the PWM CSC-based system, while other related control objectives such as MPPT, voltage balance control, active and reactive power control, are already thoroughly illustrated in the previous chapters, thus not repeated here.

\subsubsection{Simulation investigation}

Figure 6-5 illustrates the simulated performance of the two-CSC system under the optimized control scheme. $i_{g 1 \_a b c}$ and $i_{g 2 \_a b c}$ represent the three-phase load currents for Module \#1 and \#2; $I_{d c}$ is the dc-link current of the two-CSC system.

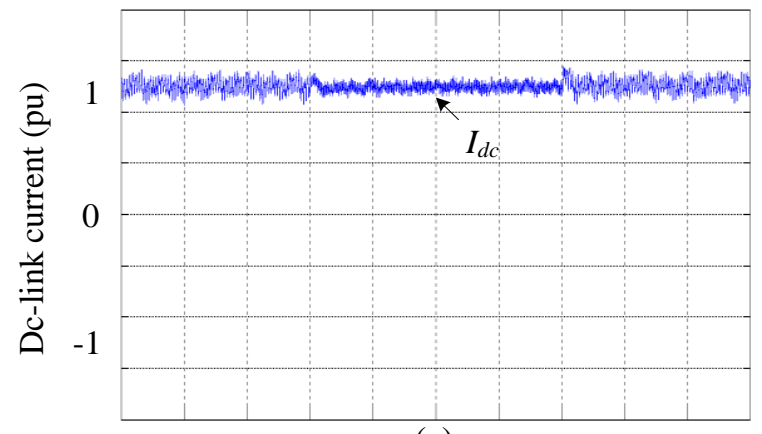

(a)

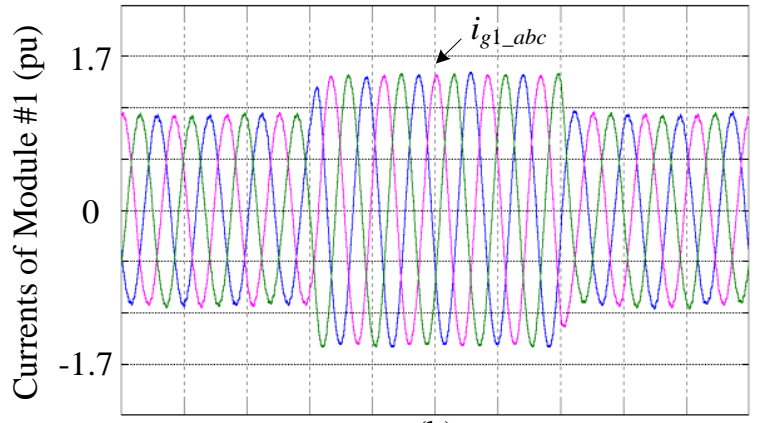

(b)

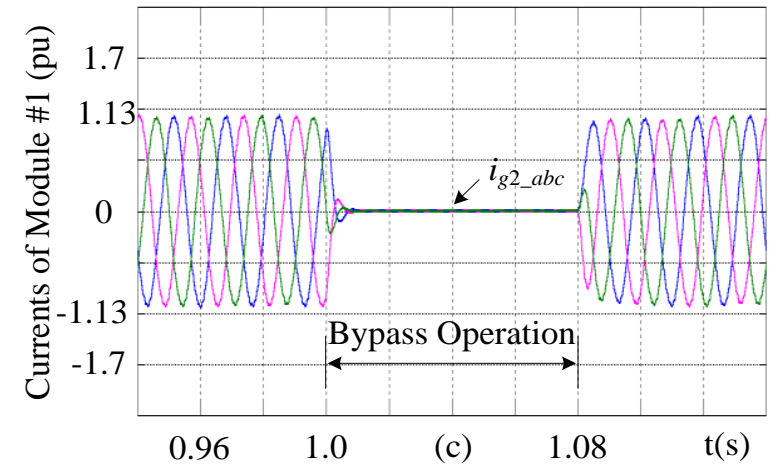

Figure 6-5 Simulated performance of the two-CSC system under optimized control. 
Before $t=1 \mathrm{~s}$, Module \#1 and \#2 are operating under a derating condition with $i_{g 1 \_a b c}=i_{g 2 \_a b c}$ $=0.707 \mathrm{pu}$. The dc-link current is controlled at a rated value, that is $I_{d c}=220 \mathrm{~A}(1 \mathrm{pu})$. At $t=1 \mathrm{~s}$, Module \#2 is purposely bypassed to lower the operation loss. At the same time, the control system reallocates the power of the two-CSC system that Module \#1 is now carrying the total power. The resultant load current of Module \#1 $i_{g 1 \_a b c}$ is decreasing from $0.7 \mathrm{pu}$ to 0 , while $i_{g 2 \_a b c}$ is increasing from $0.7 \mathrm{pu}$ to $1 \mathrm{pu}$ as shown in Figure 6-5 (b). At $t=1.08 \mathrm{~s}$, the control system purposely reactivates Module \#2 to emulate the situation when the available power of a wind farm is increased to the number that the bypassed CSC needs be switches in again in practice. As shown in Figure 6-5 (b) and (c), the load currents are changing accordingly. In the process, the dc-link current $I_{d c}$ is controlled to be fixed as shown in Figure 6-5 (a).

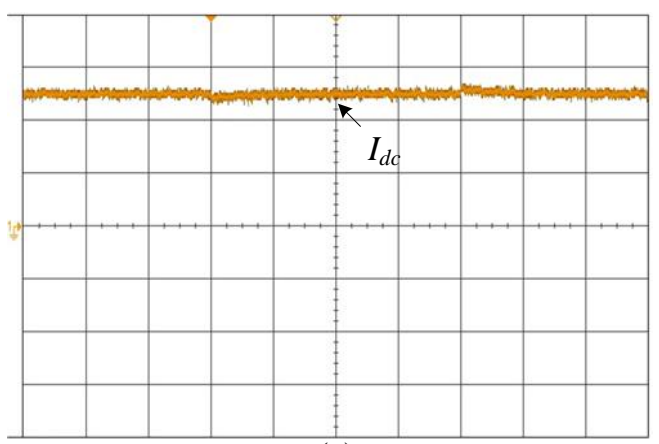

(a)

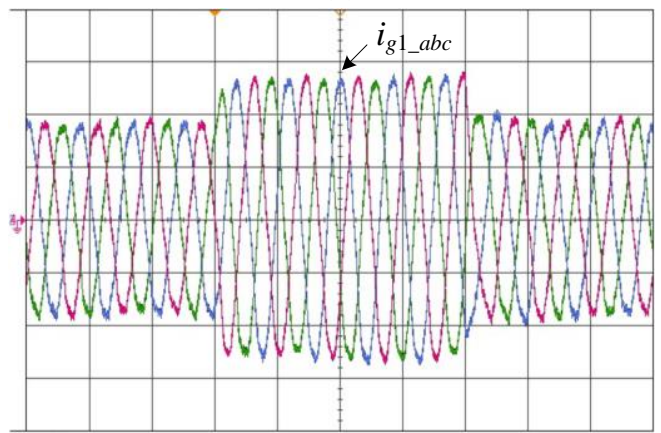

(b)

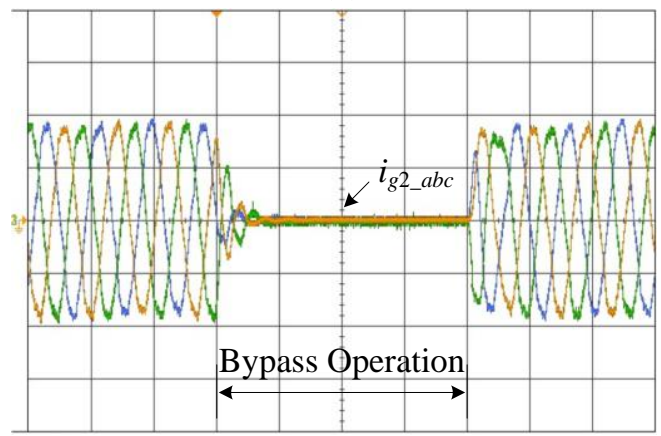

(c)

Figure 6-6 Experimental performance of the optimized strategy. 


\subsubsection{Experimental verification}

Figure 6-6 illustrates the lab-scaled experimental performance of the optimized scheme. Figure 6-6 (a) shows dc-link currents and neutral current. $I_{d c}$ : 2 A/div. Time: $20 \mathrm{~ms} / \mathrm{div}$; (b) load currents of Module \#1. $i_{g 1 \_a b c}$ : 2 A/div. Time: $20 \mathrm{~ms} / \mathrm{div}$; and (c) load currents of Module \#2. $i_{g 2 \_a b c}: 2 \mathrm{~A} / \mathrm{div}$. Time: $20 \mathrm{~ms} / \mathrm{div}$. Before time $t$, the dc-link current is controlled at a rated value, that is $I_{d c}=5 \mathrm{~A}(1 \mathrm{pu})$ as shown in Figure 6-6 (a), while Module \#1 and \#2 are purposely set to a derating operating point with $i_{g 1 \_a b c}=i_{g 2 \_a b c}=0.707 \mathrm{pu}$. At time $t$, Module \#2 is purposely bypassed to lower the operation loss. At the same time, the control system reallocates the power of the two-CSC system that Module \#1 is now carrying all the power. The resultant load current of Module \#1 $i_{g 1 \_a b c}$ is reducing from 0.7 pu to 0 , while $i_{g 2 \_a b c}$ is increasing from 0.7 pu to 1 pu as shown in Figure 6-6. 80 milliseconds later, the control system purposely reactivates Module \#2 to emulate the situation when the available power of a wind farm is increased to the number that the bypassed CSC needs to be employed again in practice. As shown in Figure 6-6 (b) and (c), the load currents are changing accordingly. In the whole process, the dc-link current $I_{d c}$ is controlled to be constant as shown in Figure 6-6 (a). Therefore, all the control objectives can be ensured.

Figure 6-7 shows the corresponding gating signals for Module \#1 and \#2. For simplicity, only two signals are illustrated here. Figure 6-7 (a) shows that during the bypass operation of Module \#2, all the devices are keeping turning on. The gating signal of the same device in Module \#1, on the other hand, is modulating but with an increased modulation index as shown in Figure 6-7 (b).

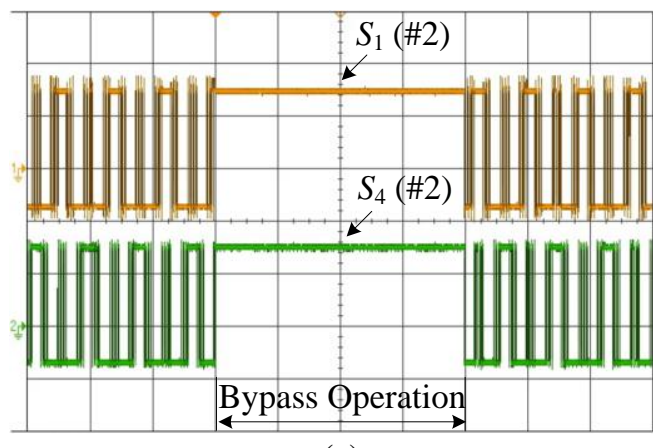

(a)

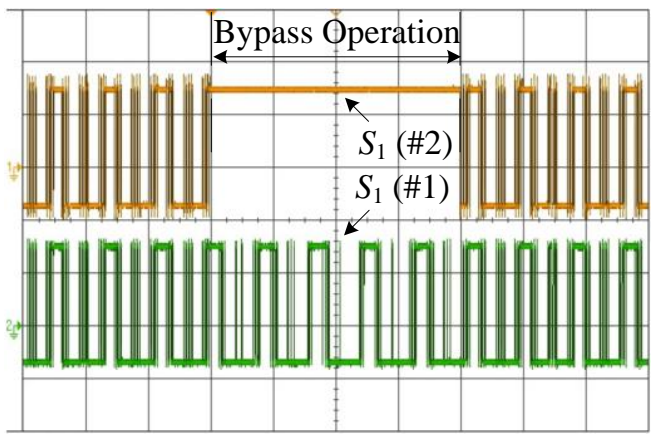

(b)

Figure 6-7 Gating Signals of switching devices.

Note that the experimental results shown above could not give an accurate cost and loss savings mainly due to the limitation of the lab-scaled experimental platform. First, the 
verification of the cost saving on the onshore CSC needs at least 10 series-connected CSCs which is too expensive to build. Thus, the resultant loss saving on the 10-CSC model could not be verified either based on the lab-scaled setup where only 2 CSCs are employed. Second, the lab-scaled setup cannot give an accurate date of loss saving by the bypass operation compared with that in the simulation (Table 6-2). The switches of the CSC used in the lab-scaled setup are three integrated modules (SKM300GBD12T4) where rated voltage/current is $1200 \mathrm{~V} / 300 \mathrm{~A}$, while the number for the MFT is $200 \mathrm{~V} / 10$ A. Thus, the lab-scaled experiment has to be conducted under a low current that the resultant operation loss saving on the CSC by the proposed strategy is inaccurate, and thus not presented here. In summary, the experiments are mainly for verification of the proposed strategy, while its benefits in terms of cost and loss shown in Table 6-2 are provided based on simulation.

\subsection{Conclusions}

In this chapter, an optimized control strategy is proposed for the CSC-based series-connected offshore wind farm with lower cost and higher efficiency. First, a nominal number of grid-side series-connected CSCs is optimized that a lower cost (10\% cost off) and a higher efficiency (8\% loss off) are achieved under rated condition. Second, a bypass operation is proposed for the onshore series-connected CSCs that the operation loss under derating conditions is reduced. The reduction in operation loss of onshore CSCs is varying depending on the maximum mismatch, different combinations of the wind speed at each turbine, and the bypass methods. Third, all the control objectives achieved in conventional scheme are ensured as the dc-link current of the system is maintained. In summary, the optimized strategy features higher efficiency and lower cost with all the control objectives of a wind farm being ensured simultaneously.

Both simulation and experiments are provided to verify the effectiveness of the proposed optimized strategy. 


\section{Chapter 7}

\section{Conclusions}

In this dissertation, a CSC-based series-connected offshore wind farm configuration is studied. The main topics include new configurations, advanced modulation scheme, and optimal system control schemes. In this chapter, the main contributions received and the future work expected are summarized.

\subsection{Contributions and conclusions}

The main contributions and conclusions of this research work are summarized as follows.

(1) New configurations have been proposed for current CSC-based series-connected offshore wind farms with smaller size and higher reliability.

Modular medium-frequency transformer (MFT)-based configurations are proposed for medium-voltage (MV) and low-voltage (LV) turbine-based offshore wind farms, respectively. Compared with commercial configurations, the proposed ones give significant cost saving thanks to the elimination of the bulky and costly offshore substation. Compared with existing seriesconnected configurations, the proposed ones feature smaller size and weight and higher reliability thanks to the adaption of the modular MFTs-based converter.

(2) A voltage/current balancing control scheme has been proposed for the offshore converters of the MV/LV configuration.

The front-end converter for MV-based configuration is a modular converter with series input and output, while it is parallel input and series output for LV-based configuration. In practice, tolerance of the components of the modular converter exists, leading to uneven distribution of power among the constituent modules. To solve this issue, a voltage balance control is proposed for the MV-based configuration and a current balance control is proposed for the LV-based one. With the help of the balancing scheme, an equal power distribution is ensured for both configurations. 
(3) An advanced SVM has been proposed for grid-side CSCs with superior low-order harmonics performance.

An advanced modulation scheme, natural sampling space vector modulation (NS-SVM), is proposed for grid-side CSCs. Compared with conventional SVM, NS-SVM preserves same dynamic performance and switching/sampling frequency, but features superior low-order harmonics performance. Also, the optimal space vector sequence based on NS-SVM in terms of low-order harmonics performance is investigated and designed for grid-side CSCs.

(4) An optimal dc-link current control has been proposed for the offshore wind farm with bipolar HVDC system.

First, the performance of the CSC-based configuration under bipolar operation mode is investigated. Compared with monopolar operation mode, bipolar operation gives lower insulation level, thus enhancing system reliability along with cost reduction. Second, an optimal dc-link current control is proposed, based on which the bipolar system is equivalent to two independent monopolar systems. The resultant efficiency and flexibility are improved.

(5) A power balancing control has been proposed for grid-side series-connected CSCs.

Equal power distribution among grid-side series-connected CSCs should be ensured for safety operation. In this thesis, the possible imbalance of power among series-connected CSCs is investigated and quantitatively defined. Accordingly, a power balancing scheme is proposed and verified that an equal distribution of power among the series-connected CSCs is ensured in the full operation range.

(6) An optimal control strategy has been proposed for the offshore wind farm with reduced cost and increased efficiency.

An optimized control strategy is proposed for the CSC-based offshore wind farm with lower cost and higher efficiency. First, the nominal number of grid-side CSCs is optimized, based on which a lower cost as well as a higher efficiency under rated condition is obtained. Second, a bypass operation is introduced to the series-connected CSCs. The efficiency under derating conditions is improved.

(7) Simulation models have been proposed for the system investigation. 
A complete system model, including the wind turbine, PMSG, modular MFT-based converters, series-connected MV CSC, and the grid, are built. The performance of the proposed configuration, modulation scheme, and control strategies are investigated based on the model.

(8) Experimental verifications on laboratory prototypes have been conducted.

In this thesis, two experimental platforms are used. One is the lab-scale platform designed for verification of the performance of the proposed configurations and the effectiveness of the proposed control schemes with all the algorithms implemented based on dSPACE DS1103. The other is a 10-kVA back-to-back CSC used to verify the performance of the proposed modulation scheme that the algorithms are implemented based on DSP (TMS320F28335) and FPGA (EP4CE10E22C8).

\subsection{Future work}

The following work is suggested for future research.

\section{Current source converter-based modular multilevel converter.}

Multilevel operation benefits the system in terms of lower switching frequencies, smaller dclink inductors, and higher quality of grid injected current. Thus, novel modulation schemes or new configurations helping obtain a multilevel operation of the CSCs are worth investigation. 


\section{Appendix}

\section{System Parameters in Simulations and}

\section{Experiments}

Detailed system ratings, generator parameters and converter parameters for simulations and experiments are listed in the following table. The pictures of Experimental Setups are given in Figure A-1 and Figure A-2.

Table A-1 Simulation and Experimental Parameters

\begin{tabular}{|c|c|c|c|c|}
\hline \multirow{2}{*}{ Parameters } & \multicolumn{2}{|c|}{ Simulation } & \multicolumn{2}{|c|}{ Experiment } \\
\hline & SI & $\mathrm{pu}$ & SI & $\mathrm{pu}$ \\
\hline \multicolumn{5}{|c|}{ System Rating } \\
\hline Nominal Power & $2 \times 1 \mathrm{MW}$ & 1.0 & $2 \times 650 \mathrm{~W}$ & 1.0 \\
\hline Grid Voltage & $4160 \mathrm{~V}$ & 1.0 & $208 \mathrm{~V}$ & 1.0 \\
\hline DC-link Current & $220 \mathrm{~A}$ & 1.0 & $8 \mathrm{~A}$ & 1.0 \\
\hline Grid/Load Current & $138 \mathrm{~A}$ & 1.0 & $3.5 \mathrm{~A}$ & 1.0 \\
\hline Frequency & $60 \mathrm{~Hz}$ & 1.0 & $60 \mathrm{~Hz}$ & 1.0 \\
\hline \multicolumn{5}{|c|}{ PMSG } \\
\hline \multicolumn{3}{|c|}{ MV PMSG } & \multirow{10}{*}{\multicolumn{2}{|c|}{$\begin{array}{c}\text { Voltage Source } \\
\text { Supply }\end{array}$}} \\
\hline Nominal Voltage & \multicolumn{2}{|c|}{$4000 \mathrm{~V}$} & & \\
\hline synchronous Inductance & \multicolumn{2}{|c|}{$0.4 \mathrm{pu}$} & & \\
\hline Rated Speed & \multicolumn{2}{|c|}{$400 \mathrm{rpm}$} & & \\
\hline Number of Pole pairs & \multicolumn{2}{|c|}{8} & & \\
\hline \multicolumn{3}{|c|}{ LV PMSG } & & \\
\hline Nominal Voltage & \multicolumn{2}{|c|}{$690 \mathrm{~V}$} & & \\
\hline synchronous Inductance & \multicolumn{2}{|c|}{$0.45 \mathrm{pu}$} & & \\
\hline Rated Speed & \multicolumn{2}{|c|}{$22.5 \mathrm{rpm}$} & & \\
\hline Number of Pole pairs & \multicolumn{2}{|c|}{26} & & \\
\hline \multicolumn{5}{|c|}{ Generators-side converters } \\
\hline \multicolumn{5}{|c|}{ MFT-based converter for MV-based system } \\
\hline Number of Modules & \multicolumn{2}{|c|}{5} & \multicolumn{2}{|c|}{3} \\
\hline Turn Ratios of Transformers & \multicolumn{2}{|c|}{$\begin{array}{c}1: 1 \\
1: 1.005 \\
1: 1.01 \\
1: 1.015 \\
1: 1.02\end{array}$} & \multicolumn{2}{|c|}{$\begin{array}{c}1: 1 \\
1: 1 \\
1: 1 \\
\text { Tolerance } \pm 2 \%\end{array}$} \\
\hline Input Capacitor & $200 \mathrm{uF}$ & 2.0 & $1000 \mathrm{uF}$ & 2.0 \\
\hline Switching Frequency & $1200 \mathrm{~Hz}$ & 1.0 & $1200 \mathrm{~Hz}$ & 1.0 \\
\hline
\end{tabular}




\begin{tabular}{|c|c|c|c|c|}
\hline \multicolumn{5}{|c|}{ MFT-based converter for LV-based system } \\
\hline Number of Modules & \multicolumn{2}{|c|}{6} & \multicolumn{2}{|c|}{3} \\
\hline Turn Ratios of Transformers & & & Tolera & $\begin{array}{l}1 \\
1 \\
1 \\
c e \pm 2 \%\end{array}$ \\
\hline Input Capacitor & $200 \mathrm{uF}$ & 2.0 & $1000 \mathrm{uF}$ & 2.0 \\
\hline Switching Frequency & $1200 \mathrm{~Hz}$ & 1.0 & $1200 \mathrm{~Hz}$ & 1.0 \\
\hline \multicolumn{5}{|c|}{ Grid-side converters } \\
\hline \multicolumn{5}{|c|}{ PWM CSC \#1 } \\
\hline DC-link Inductor $\left(L_{d c}\right)$ & $45 \mathrm{mH}$ & 1.0 & $40 \mathrm{mH}$ & 0.9 \\
\hline Grid-side inductor $\left(L_{g}\right)$ & $4.5 \mathrm{mH}$ & 0.1 & $5 \mathrm{mH}$ & 0.11 \\
\hline Grid-side Capacitor $\left(\mathrm{C}_{f}\right)$ & $77 \mathrm{uF}$ & 0.5 & $90 \mathrm{uF}$ & 0.58 \\
\hline Switching Frequency $\left(f_{s w}\right)$ & $540 \mathrm{~Hz}$ & 1.0 & $540 \mathrm{~Hz}$ & 1.0 \\
\hline Modulation Scheme & \multicolumn{2}{|c|}{ SVM } & \multicolumn{2}{|c|}{ SVM } \\
\hline \multicolumn{5}{|c|}{ PWM CSC \#2 } \\
\hline DC-link Inductor $\left(L_{d c}\right)$ & $45 \mathrm{mH}$ & 1.0 & $40 \mathrm{mH}$ & 0.9 \\
\hline Grid-side inductor $\left(L_{g}\right)$ & $4.5 \mathrm{mH}$ & 0.1 & $5 \mathrm{mH}$ & 0.11 \\
\hline Grid-side Capacitor $\left(\mathrm{C}_{f}\right)$ & $77 \mathrm{uF}$ & 0.5 & $90 \mathrm{uF}$ & 0.58 \\
\hline Switching Frequency $\left(f_{s w}\right)$ & $540 \mathrm{~Hz}$ & 1.0 & $540 \mathrm{~Hz}$ & 1.0 \\
\hline Modulation Scheme & \multicolumn{2}{|c|}{ SVM } & \multicolumn{2}{|c|}{ SVM } \\
\hline \multicolumn{5}{|c|}{ Cables } \\
\hline $\begin{array}{l}\text { Length of Cable Between } \\
\text { Adjacent Offshore Converters } \\
\left(l_{c \_n}\right)\end{array}$ & \multicolumn{2}{|c|}{$1 \mathrm{~km}$} & \multicolumn{2}{|c|}{$50 \mathrm{~m}$} \\
\hline $\begin{array}{l}\text { Length of Cable Between } \\
\text { Offshore and Onshore } \\
\text { Converters }\left(l_{c_{-} T}\right)\end{array}$ & \multicolumn{2}{|c|}{$50 \mathrm{~km}$} & \multicolumn{2}{|c|}{$50 \mathrm{~m}$} \\
\hline Resistance Per Unit Length & \multicolumn{2}{|c|}{$0.2 \mathrm{ohms} / \mathrm{km}$} & \multicolumn{2}{|c|}{$3 \mathrm{ohms} / \mathrm{km}$} \\
\hline Inductance Per Unit Length & \multicolumn{2}{|c|}{$1.4 \mathrm{mH} / \mathrm{km}$} & \multicolumn{2}{|c|}{$0.35 \mathrm{mH} / \mathrm{km}$} \\
\hline Capacitance Per Unit Length & \multicolumn{2}{|c|}{$0.142 \mu \mathrm{F} / \mathrm{km}$} & \multicolumn{2}{|c|}{$0.12 \mu \mathrm{F} / \mathrm{km}$} \\
\hline \multicolumn{5}{|c|}{ Medium-frequency Transformer } \\
\hline Rated Power & & & & VA \\
\hline Rated Input/Output Voltage & 100 & & 200 & $00 \mathrm{~V}$ \\
\hline Rated Current & & & & A \\
\hline Leakage Inductance & & & & $\mu \mathrm{H}$ \\
\hline
\end{tabular}



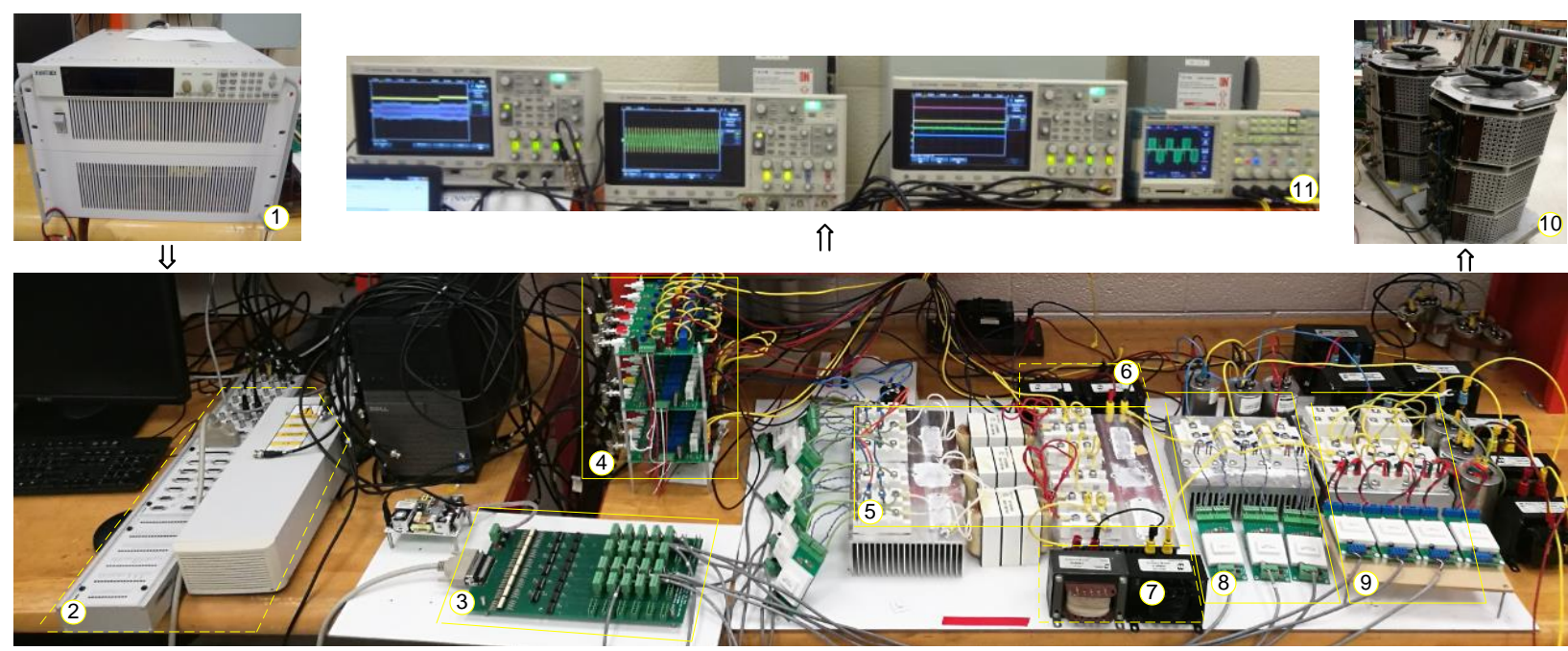

Figure A-1 Photo of the lab-scaled platform.

(1) Constant voltage supply. (2) dSPACE DS1103. (3) Driver interface board. (4) Voltage/current sensor boards. (5) MFT-based converter. (6) DC-link inductor for Module \#1. (7) DC-link inductor for Module \#2. (8) PWM CSC \#1. (9) PWM CSC \#2. (10) Grid-side transformers. (11) Oscilloscopes.

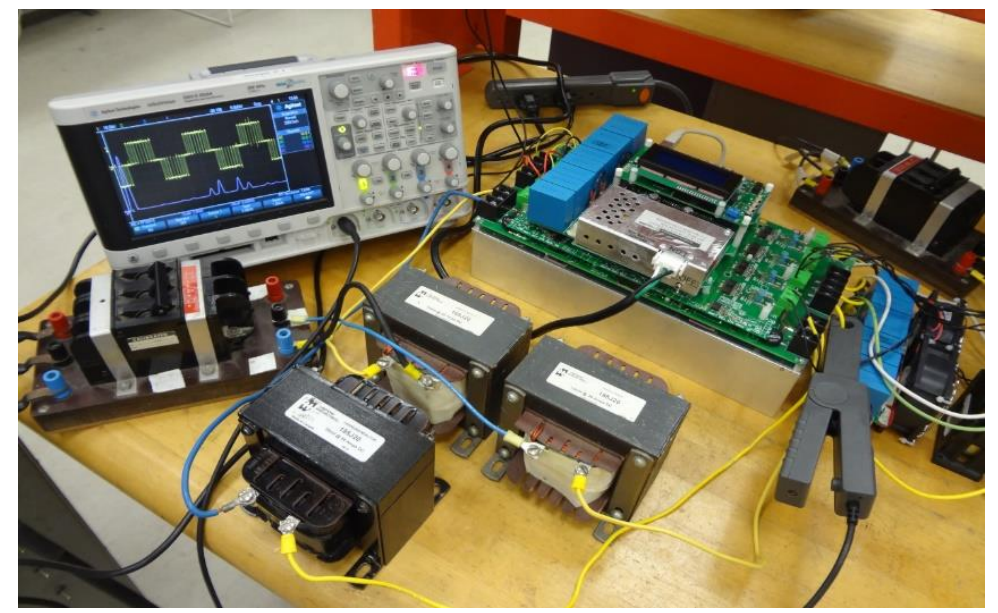

Figure A-2 10-kVA back-to-back PWM CSC converter. 


\section{References}

[1] Global Wind Energy Outlook 2010, Global Wind Energy Council, London, U.K., 2010.

[2] B. Wu; Y. Lang; N. Zargari; S. Kouro, Power Conversion and Control of Wind Energy Systems. Wiley-IEEE Press, 2011.

[3] Offshore Turbine Database, 4 C Offshore, May 2014. [Online]. Available: http://www. 4 coffshore.com.

[4] S. Lundberg, "Evaluation of Wind Farm Layouts," EPE Journal. Power Electronics and Drives, vol. 16, no. 1, pp. 14-21, 2006.

[5] N. Flourentzou, V. Agelidis, and G. Demetriades, "VSC-Based HVDC Power Transmission Systems: An Overview," IEEE Transactions on Power Electronics, vol. 24, no. 3, pp. 592-602, Mar. 2009.

[6] F. Blaabjerg and K. Ma "Future on Power Electronics for Wind Turbine Systems", IEEE Journal of Emerging and Selected Topics in Power Electronics, vol. 1, no. 3, pp. 139-152, Sept. 2013.

[7] K. Friedrich, "Modern HVDC PLUS Application of VSC in Modular Multilevel Converter Topology," in Proc. IEEE International Symposium on Industrial Electronics, Bari, 2010, pp. 38073810.

[8] D. Das, J. Pan, and S. Bala, "HVDC Light for Large Offshore Wind Farm Integration," IEEE Power Electronics and Machines in Wind Applications, Denver, CO, 2012, pp. 1-7.

[9] O. Anaya-Lara; N. Jenkins; J. Ekanayake; P. Cartwright; M. Hughes and M. Hughes, Wind Energy Generation: Modeling and Control. Wiley, 2009.

[10] P. Bresesti, W. L. Kling, R. L. Hendriks and R. Vailati, "HVDC Connection of Offshore Wind Farms to the Transmission System," IEEE Transactions on Energy Conversion, vol. 22, pp. 37-43, Mar. 2007.

[11] Z. Chen, J. Guerrero, and F. Blaabjerg, "A Review of the State of the Art of Power Electronics for Wind Turbines," IEEE Transactions on Power Electronics, vol. 24, no. 8, pp. 1859-1875, Aug. 2009.

[12] X. Koutiva, T. Vrionis, N. A. Vovos and G. B. Giannakopoulos, "Optimal Integration of an Offshore Wind Farm to a Weak AC Grid," IEEE Transactions on Power Delivery, vol. 21, pp. 987-994, Apr. 2006.

[13] S. M. Muyeen, R. Takahashi and J. Tamura, "Operation and Control of HVDC-Connected Offshore Wind Farm," IEEE Transactions on Sustainable Energy, vol. 1, pp. 30-37, Apr. 2010. 
[14] S. V. Bozhko, R. Blasco-Gimenez, R. Li, J. C. Clare and G. M. Asher, "Control of Offshore DFIGBased Wind Farm Grid with Line-Commutated HVDC Connection," IEEE Transactions on Energy Conversion, vol. 22, pp. 71-78, Mar. 2007.

[15] S. Bozhko, G. Asher, R. Li, J. Clare and L. Yao, "Large Offshore DFIG Based Wind Farm with Line-Commutated HVDC Connection to the Main Grid: Engineering Studies," IEEE Transactions on Energy Conversion, vol. 23, pp. 119-127, Mar. 2008.

[16] N. Kirby, L. Xu, M. Luckett and W. Siepmann, "HVDC Transmission for Large Offshore Wind Farms," Power Engineering Journal, vol. 16, pp. 135-141, Jun. 2002.

[17] L. Xu, L. Yao and C. Sasse, "Power Electronics Options for Large Wind Farm Integration: VSCBased HVDC Transmission," IEEE Power Systems Conference and Exposition, Atlanta, GA, 2006, pp. 760-767.

[18] M. Popat, "Current Source Converter Based Offshore Wind Farm: Configuration and Control" (2013). Theses and dissertations. Paper 1525.

[19] M. P. Bahrman and B. K. Johnson, "The ABCs of HVDC Transmission Technologies," IEEE Power and Energy Magazine, vol. 5, no. 2, pp. 32-44, March-April 2007.

[20] J. M. Carrasco et al., "Power-Electronic Systems for the Grid Integration of Renewable Energy Sources: A Survey," IEEE Transactions on Industrial Electronics, vol. 53, no. 4, pp. 1002-1016, Jun. 2006.

[21] M. Popat, B. Wu and N. R. Zargari, "A Novel Decoupled Interconnecting Method for CurrentSource Converter-Based Offshore Wind Farms," IEEE Transactions on Power Electronics, vol. 27, pp. 4224-4233, Oct. 2012.

[22] B. Wu and M. Narimani, High-Power Converters and AC Drives. Wiley-IEEE Press, 2017.

[23] F. Blaabjerg, Z. Chen, and S. Kjaer, "Power Electronics as Efficient Interface in Dispersed Power Generation Systems," IEEE Transactions on Power Electronics, vol. 19, no. 5, pp. 1184-1194, Sept. 2004.

[24] M. Liserre, R. Cardenas, M. Molinas, and J. Rodriguez, "Overview of Multi-MW Wind Turbines and Wind Parks," IEEE Transactions on Industrial Electronics, vol. 58, no. 4, pp. 1081-1095, Apr. 2011.

[25] M. Perez, S. Bernet, J. Rodriguez, S. Kouro, and R. Lizana, "Circuit Topologies, Modeling, Control Schemes, and Applications of Modular Multilevel Converters," IEEE Transactions on Power Electronics, vol. 30, no. 1, pp. 4-17, Jan. 2015. 
[26] S. Debnath, J. Qin, B. Bahrani, M. Saeedifard, and P. Barbosa, "Operation, Control, and Applications of the Modular Multilevel Converter: A Review," IEEE Transactions on Power Electronics, vol. 30, no. 1, pp. 37-53, Jan. 2015.

[27] C. Meyer, M. Hoing, A. Peterson, and R. W. De Doncker, "Control and Design of DC Grids for Offshore Wind Farms," IEEE Transactions on Industrial Applications, vol. 43, no. 6, pp. 1475-1482, Nov. 2007.

[28] J. Robinson, D. Jovcic, and G. Joos, "Analysis and Design of an Offshore Wind Farm Using a MV DC Grid," IEEE Transactions on Power Delivery, vol. 25, no. 4, pp. 2164-2173, Oct. 2010.

[29] A. Prasad, P. Ziogas, and S. Manias, "Analysis and Design of a Three-Phase Offline DC-DC Converter with High-Frequency Isolation," IEEE Transactions on Industrial Applications, vol. 28, no. 4, pp. 824-832, Jul. 1992.

[30] W. Chen, A. Q. Huang, C. Li, G. Wang, and W. Gu, "Analysis and Comparison of Medium Voltage High Power DC/DC Converters for Offshore Wind Energy Systems," IEEE Transactions on Power Electronics, vol. 28, no. 4, pp. 2014-2023, Apr. 2013.

[31] A. Garcés and M. Molinas, "A Study of Efficiency in a Reduced Matrix Converter for Offshore Wind Farms," IEEE Transactions on Industrial Electronics, vol. 59, no. 1, pp. 184-193, Jan. 2012.

[32] S. Nishikata and F. Tatsuta, "A New Interconnecting Method for Wind Turbine/Generators in a Wind Farm and Basic Performances of the Integrated System," IEEE Transactions on Industrial Electronics, vol. 57, no. 2, pp. 468-475, Feb. 2010.

[33] V. Yaramasu, B. Wu, P. C. Sen, S. Kouro and M. Narimani, "High-Power Wind Energy Conversion Systems: State-of-the-Art and Emerging Technologies," Proceedings of the IEEE, vol. 103, no. 5, pp. 740-788, May 2015.

[34] N. Kirby, L. Xu, M. Luckett and W. Siepmann, "HVDC Transmission for Large Offshore Wind Farms," Power Engineering Journal, vol. 16, pp. 135-141, Jun. 2002.

[35] Offshore wind connections. [Online]. Available: http://new.abb.com/systems/offshore-windconnections.

[36] Q. Wei, B. Wu, D. Xu and N. R. Zargari, "A Medium-Frequency Transformer-Based Wind Energy Conversion System Used for Current-Source Converter-Based Offshore Wind Farm," IEEE Transactions on Power Electronics, vol. 32, no. 1, pp. 248-259, Jan. 2017. 
[37] Y. Xia, J. Fletcher, S. Finney, K. Ahmed, and B. Williams, "Torque Ripple Analysis and Reduction for Wind Energy Conversion Systems Using Uncontrolled Rectifier and Boost Converter," IET Renewable Power Generation, vol. 5, no. 5, pp. 377-386, Sept. 2011.

[38] J. Dai, D. Xu, and B. Wu, "A Novel Control Scheme for Current-Source Converter-Based PMSG Wind Energy Conversion Systems," IEEE Transactions on Power Electronics, vol. 24, no. 4, pp. 963972, Apr. 2009.

[39] R. Giri, R. Ayyanar and E. Ledezma, "Input-Series and Output-Series Connected Modular DC-DC Converters with Active Input Voltage and Output Voltage Sharing," IEEE Applied Power Electronics Conference and Exposition, 2004, pp. 1751-1756.

[40] M. Popat, B. Wu, F. Liu and N. Zargari, "Coordinated Control of Cascaded Current Source Converter Based Offshore Wind Farms," IEEE Transactions on Sustainable Energy, vol. 3, no. 3, pp. 557-565, Jul. 2012.

[41] J. W. Kolar, G. Ortiz, Solid-State Transformers, Plenary Session Presentation at the IEEE International Power Electronics and Applications Conference and Exhibition, Shanghai, 2014, pp. 58.

[42] D. Dujic, C. Zhao, A. Mester, J. K. Steinke, M. Weiss, S. LewdeniSchmid, P. Stefanutti, and T. Chaudhuri, "Power Electronic Traction Transformer-Low Voltage Prototype," IEEE Transactions on Power Electronics, vol. 28, no. 12, pp. 5522-5534, Dec. 2013.

[43] C. Zhao, D. Dujic, A. Mester, J. K. Steinke, M. Weiss, S. LewdeniSchmid, P. Stefanutti, and T. Chaudhuri, "Power Electronic Traction Transformer-Medium Voltage Prototype," IEEE Transactions on Power Electronics, vol. 6, no. 7, pp. 3257-3268, Jul. 2014.

[44] M. A. Bahmani, T. Thiringer, A. Rabiei and T. Abdulahovic, "Comparative Study of a Multi-MW High-Power Density DC Transformer with an Optimized High-Frequency Magnetics in All-DC Offshore Wind Farm," IEEE Transactions on Power Delivery, vol. 31, no. 2, pp. 857-866, Apr. 2016.

[45] E. Agheb and H. Hoidalen, "Medium Frequency High Power Transformers, State of Art and Challenges," International Conference on Renewable Energy Research and Applications, Nagasaki, 2012, pp. 1-6.

[46] G. Ortiz, J. Biela, D. Bortis and J. W. Kolar, "1 Megawatt, 20 kHz, Isolated, Bidirectional 12kV to 1.2kV DC-DC Converter for Renewable Energy Applications," International Power Electronics Conference, Sapporo, 2010, pp. 3212-3219. 
[47] R. Giri, V. Choudhary, R. Ayyanar, and N. Mohan, "Common-Duty Ratio Control of Input-Series Connected Modular DC-DC Converters with Active Input Voltage and Load-Current Sharing," IEEE Transactions on Industrial Applications, vol. 42, no. 4, pp. 1101-1111, Jul-Aug. 2006.

[48] Q. Wei, B. Wu, D. Xu and N. R. Zargari, "Model Predictive Control of Capacitor Voltage Balancing for Cascaded Modular DC-DC Converters," IEEE Transactions on Power Electronics, vol. 32, no. 1, pp. 752-761, Jan. 2017.

[49] ABB XPLE Submarine Cables. [Online]. Available: http://www.abb.com/product/us/9AAC30200079.aspx.

[50] J. D. Glover, M. S. Sarma and T. J. Overbye, Power System Analysis and Design, Fifth Edition, Global Engineering, 2012.

[51] N. Chaudhuri, B. Chaudhuri, R. Majumder and A. Yazdani, Multi-Terminal Direct-Current Grids: Modeling, Analysis, and Control. Wiley-IEEE Press, 2014.

[52] Q. Wei, B. Wu, D. Xu and N. R. Zargari, "Natural Sampling SVM Based Method for CommonMode Voltage Reduction in Medium-Voltage Current Source Rectifier," IEEE Transactions on Power Electronics, vol. 32, no. 10, pp. 7553-7560, Oct. 2017.

[53] M. Saeedifard, A. Bakhshai, and G. Joos, "Low Switching Frequency Space Vector Modulators for High Power Multimodule Converters," IEEE Transactions on Power Electronics, vol. 20, no. 6, pp. 1310-1318, Nov. 2005.

[54] B. Abdul Rahiman, G. Narayanan, and V. T. Ranganathan, "Modified SVPWM Algorithm for Three Level VSI with Synchronized and Symmetrical Waveforms," IEEE Transactions on Industrial Electronics, vol. 54, no. 1, pp. 486-494, Feb. 2007.

[55] D. G. Holmes, "A Unified Modulation Algorithm for Voltage and Current Source Inverters Based on AC-AC Matrix Converter Theory," IEEE Transactions on Industrial Applications, vol. 28, no. 1, pp. 31-40, Jan-Feb. 1992.

[56] D. N. Zmood and D. G. Holmes, "A Generalised Approach to the Modulation of Current Source Inverters," IEEE Power Electronics Specialists Conference, Fukuoka, 1998, pp. 739-745.

[57] O. Ojo and S. Vanaparthy, "Carrier-Based Discontinuous PWM Modulation for Current Source Converters," IEEE Industry Applications Conference, 2004, pp. 2224-2231.

[58] T. Halkosaari and H. Tuusa, "Optimal Vector Modulation of a PWM Current Source Converter According to Minimal Switching Losses," IEEE Power Electronics Specialists Conference, Galway, 2000, pp. 127-132. 
[59] E. P. Wiechmann, R. P. Burgos, and J. Holtz, "Active Front-End Converter for Medium-Voltage Current Source Drives Using Sequential-Sampling Synchronous Space-Vector Modulation," IEEE Transactions on Industrial Electronics, vol. 50, no. 6, pp. 1275-1289, Dec. 2003.

[60] J. D. Ma, B. Wu, N. R. Zargari, and S. C. Rizzo, "A Space Vector Modulated CSI-Based AC Drive for Multimotor Applications," IEEE Transactions on Power Electronics, vol. 16, no. 4, pp. 535-544, Jul. 2001.

[61] Y. W. Li, B. Wu, D. Xu, and N. R. Zargari, "Space Vector Sequence Investigation and Synchronization Methods for Active Front-End Rectifiers in High-Power Current-Source Drives," IEEE Transactions on Industrial Electronics, vol. 55, no. 3, pp. 1022-1034, Mar. 2008.

[62] M. F. Naguib and L. Lopes, "Harmonics Reduction in Low Switching Frequency Space Vector Modulated Current Source Converters," IEEE Power Electronics Special Conference, Rhodes, 2008, pp. 1434-1440.

[63] M. F. Naguib and L. A. C. Lopes, "Harmonics Reduction in Current Source Converters Using Fuzzy Logic," IEEE Transactions on Power Electronics, vol. 25, no. 1, pp. 158-167, Jan. 2010.

[64] L. Lopes and M. F. Naguib, "Space Vector Modulation for Low Switching Frequency Current Source Converters with Reduced Low-Order Noncharacteristic Harmonics," IEEE Transactions on Power Electronics, vol. 24, no. 4, pp. 903-910, Apr. 2009.

[65] J. Dai, Y. Lang, B. Wu, D. Xu, and N. R. Zargari, "A Multisampling SVM Scheme for Current Source Converters with Superior Harmonic Performance," IEEE Transactions on Power Electronics, vol. 24, pp. 2436-2445, Nov. 2009.

[66] Q. Wei, B. Wu, D. Xu and N. R. Zargari, "Minimization of Filter Capacitor for Medium-Voltage Current-Source Converters Based on Natural Sampling SVM," IEEE Transactions on Power Electronics, In Press.

[67] Q. Wei, B. Wu, D. Xu and N. R. Zargari, "A Natural-Sampling-Based SVM Scheme for Current Source Converter with Superior Low-Order Harmonics Performance," IEEE Transactions on Power Electronics, vol. 31, no. 9, pp. 6144-6154, Sept. 2016.

[68] Q. Wei, B. Wu, D. Xu and N. R. Zargari, "Optimal Space Vector Sequence Investigation Based on Natural Sampling SVM for Medium-Voltage Current-Source Converter," IEEE Transactions on Power Electronics, vol. 32, no. 1, pp. 176-185, Jan. 2017. 
[69] Q. Wei, B. Wu, D. Xu, and N. R. Zargari, "Power Balancing Investigation of Grid-Side SeriesConnected Current Source Inverters in Wind Energy Conversion Systems," IEEE Transactions on Industrial Electronics, In Press.

[70] PowerFlex 7000 MV Drive Brochure, Rockwell Automation Canada Inc., 2002.

[71] Aluminum Electrolytic Capacitors. [Online]. Available: https://en.tdk.eu/tdken/529362/products/product-catalog/aluminum-electrolytic-capacitors.

[72] D. Jovcic, "Offshore Wind Farm with a Series Multiterminal CSI HVDC," Electric Power System Research, vol. 78, no. 4, pp. 747-755, Apr. 2008.

[73] S. Lundberg, "Configuration Study of Large Wind Parks," Licentiate Thesis, Chalmers Univ. Technol., Dept. of Elect, Power Engineering., Goteborg, Sweden, 2003.

[74] Q. Wei, B. Wu, D. Xu and N. R. Zargari, "Bipolar Operation Investigation of PWM Current Source Converter-Based Wind Energy Conversion Systems," IEEE Transactions on Power Electronics, In Press.

[75] N. Mohan, T. M. Undeland, and W. P. Robbins, Power Electronics: Converters, Applications, and Design: Wiley, 2nd Edition, Jan. 1995.

[76] N. R. Zargari, S. C. Rizzo, Y. Xiao, H. Iwamoto, K. Satoh and J. F. Donlon, "A New Current-Source Converter Using a Symmetric Gate-Commutated Thyristor (SGCT)," IEEE Transactions on Industry Applications, vol. 37, no. 3, pp. 896-903, May-Jun 2001.

[77] Q. Wei, B. Wu, D. Xu and N. R. Zargari, "An Optimized Strategy for PWM Current Source Converter-Based Wind Conversion System with Reduced Cost and Improved Efficiency," IEEE Transactions on Power Electronics, In Press.

[78] IGCT. [Online]. Available: http://new.abb.com/semiconductors/integrated-gate-commutatedthyristors-(igct). 


\section{Glossary of Acronyms and Symbols}

\begin{tabular}{|c|c|}
\hline CSC & Current Source Converter \\
\hline DFIG & Doubly Fed Induction Generator \\
\hline EESG & Electrically Excited Synchronous Generator \\
\hline EMF & Electro-Motive Force \\
\hline FFT & Fast Fourier Transformation \\
\hline HVAC & High Voltage Alternating Current \\
\hline HVDC & High Voltage Direct Current \\
\hline LCC & Line Commutated Converter \\
\hline LV & Low-Voltage \\
\hline MFT & Medium-Frequency Transformer \\
\hline MMC & Modular Multilevel Converter \\
\hline MPPT & Maximum Power Point Tracking \\
\hline MV & Medium-Voltage \\
\hline MVAC & Medium Voltage Alternating Current \\
\hline NS-SVM & Natural Sampling Space Vector Modulation \\
\hline $\mathrm{PF}$ & Power-Factor \\
\hline PMSG & Permanent Magnet Synchronous Generators \\
\hline PU & Per Unit \\
\hline SGCT & Symmetric Gate Commutated Thyristor \\
\hline SHE & Selective Harmonics Elimination \\
\hline SQ1 & Sequence 1 \\
\hline SQ2 & Sequence 2 \\
\hline SQ3 & Sequence 3 \\
\hline
\end{tabular}




$\begin{array}{ll}\text { SQ4 } & \text { Sequence 4 } \\ \text { SQ5 } & \text { Sequence 5 } \\ \text { SQ6 } & \text { Sequence 6 } \\ \text { STATCOM } & \text { Static Synchronous Compensator } \\ \text { SVC } & \text { Static VAR Compensator } \\ \text { SVM } & \text { Space Vector Modulation } \\ \text { TPWM } & \text { Trapezoidal Pulse Width Modulation } \\ \text { UPF } & \text { Unity Power Factor } \\ \text { VSC } & \text { Voltage Source Converter } \\ \text { WECS } & \text { Wind Energy Conversion System } \\ \text { WFSC } & \text { Wind Farm Supervisory Control } \\ \text { WRIG } & \text { Wound-Rotor Induction Generator }\end{array}$

The following provides explanations to the variables that are commonly used in this dissertation. Other variables which only appear in specific sections are explained in the context where they appear.

\section{Voltages}

$\begin{array}{ll}V_{c 1}, V_{c 2} \ldots V_{c 6} & \text { input capacitor voltages of the modular MFT converter } \\ v_{a} & \text { grid phase voltage } \\ V_{i n} & \text { total input voltage of the modular MFT converter } \\ v_{a o}, v_{b o}, v_{c o} & \text { converter output voltages } \\ V_{L L} & \text { grid line-to-line voltage } \\ V_{d c 1}, \ldots V_{d c \mathrm{~m}} & \text { average input voltage of each CSC } \\ v_{d c 1}, \ldots v_{d \mathrm{~cm}} & \text { instantaneous input voltage of each CSC } \\ V_{d \mathrm{~cm} \_r e f} & \text { average input voltage reference of each CSC } \\ V_{s d}, V_{s q}, & d \text { - and } q \text {-axis components of the grid voltage } \\ V_{c d}, V_{c q} & d \text { - and } q \text {-axis components of capacitor voltages }\end{array}$




\section{Currents}

\begin{tabular}{|c|c|}
\hline$I_{d c}$ & dc-link current of the offshore wind farm \\
\hline$i_{w}$ & converter output PWM current \\
\hline$i_{g}$ & line current \\
\hline$I_{d c_{-} g e}$ & generator-side converter determined dc-link current \\
\hline$I_{d c \_g r}$ & grid-side converter determined dc-link current \\
\hline $\bar{I}_{m N}$ & average input current of the modular MFT converter \\
\hline$I_{d c \_r e f}$ & dc-link reference current \\
\hline$I_{g d \_r e f}, I_{g q \_} r e f$ & $d$-axis, $q$-axis grid current references \\
\hline$I_{w d_{-} r e f}, I_{w q_{-} r e f}$ & $d$-axis, $q$-axis converter reference current \\
\hline$I_{C_{-} U P F}$ & capacitor current under UPF \\
\hline$I_{g_{-} U P F}$ & line current under UPF \\
\hline$i_{s d}, i_{s q}$ & $d$ - and $q$-axis components of the grid currents \\
\hline$i_{c d}, i_{c q}$ & $d$ - and $q$-axis components of the capacitor currents \\
\hline$I_{d c \_g r}$ & magnitude of the minimum dc-link current \\
\hline$I_{d c_{-} \text {offshore }}$ & dc-link current determined by offshore converters \\
\hline$I_{d c \_g r i d}$ & dc-link current determined by onshore CSCs \\
\hline$I_{d c_{-} \text {rated }}$ & rated dc-link current \\
\hline
\end{tabular}

\section{Power}

$\begin{array}{ll}P_{s} & \text { grid-side active power } \\ Q_{s} & \text { grid-side reactive power } \\ Q_{r e f} & \text { grid-side reactive power reference } \\ P_{W F} & \text { Total wind power of the offshore wind farm } \\ P_{W F_{-} \text {rated }} & \text { rated power of the wind farm } \\ P_{g e_{-} \mathrm{n}} & \text { captured wind power of each turbine unit }\end{array}$



$P_{s w}$
switching loss for CSCs
$P_{\text {con }}$
conduction loss for CSCs

\section{LC components related}

$\begin{array}{ll}L_{g} & \text { grid-side equivalent line inductance } \\ L_{d c} & \text { dc-link inductance } \\ C_{f} & \text { CSC output filter capacitance } \\ C_{i n} & \text { Input capacitance of the modular MFT converter } \\ R_{g} & \text { grid-side line resistance } \\ R_{l} & \text { equivalent load of grid-side CSCs } \\ k_{1} & \text { tolerance coefficient of the capacitance } \\ k_{2} & \text { tolerance coefficient of the inductance }\end{array}$

\section{CSC modulation related}

$\begin{array}{ll}\vec{I}_{1}-\vec{I}_{6} & \text { active vectors of CSCs } \\ \vec{I}_{0} & \text { zero vector of CSCs } \\ f_{1} & \text { fundamental frequency of CSC output current } \\ \omega & \text { fundamental angular frequency of CSC output current } \\ \theta & \text { angle of a vector in the space vector plane } \\ m_{a} & \text { modulation index of CSCs } \\ T_{s} & \text { sampling period } \\ f_{s w} & \text { switching frequency } \\ f_{s p} & \text { delay angle of CSCs } \\ \alpha & \end{array}$

